\title{
Total Synthesis of the Ambigols: A Cyanobacterial Class of Polyhalogenated Natural Products
}

\author{
Tobias M. Milzarek, ${ }^{a}$ and Tobias A. M. Gulder ${ }^{{ }^{*}}$ \\ a Chair of Technical Biochemistry, Technical University of Dresden, Bergstraße 66, 01062 Dresden, Germany. E-mail: \\ tobias.gulder@tu-dresden.de
}

Supporting Information

\section{Contents}

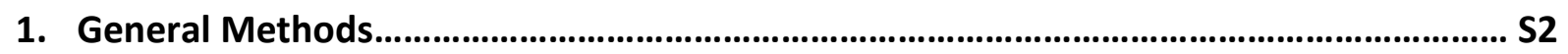

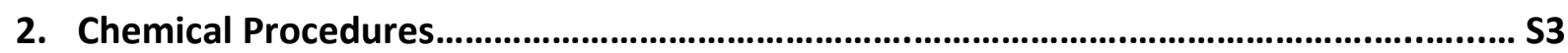

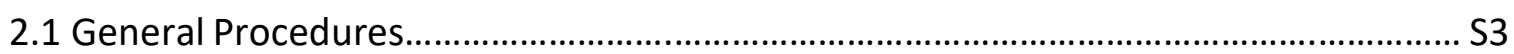

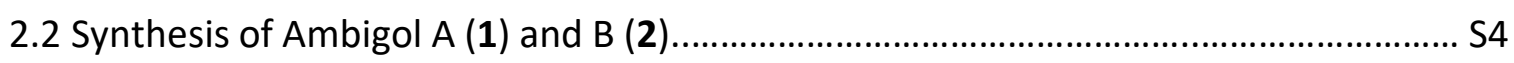

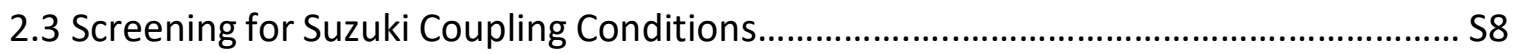

2.4 Synthesis of Ambgiol C (3) and Derivative 30.......................................................... S11

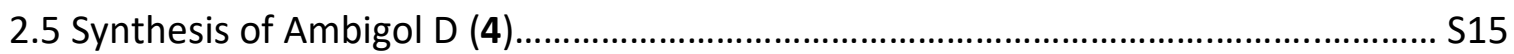

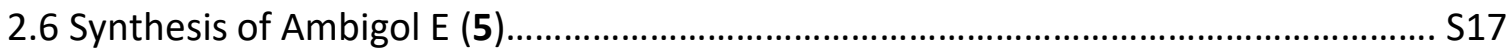

2.7 Synthesis of Strucutral Analogue 47 ...................................................................... S20

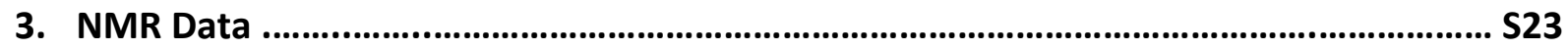

4. Analysis of Rotational Stability of Ambigol A (1) ......................................................... S79

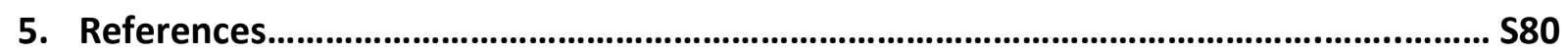




\section{General Methods}

Reagents: Solvents for HPLC and MS analysis, such as acetonitrile and methanol, were purchased from Fisher Scientific and VWR in a purity of over 99\% (HPLC-grade). Water was purified using a TKA GenPure water treatment system and deionized. Dry solvents, such as diethyl ether, dichloromethane, 1,4-dioxane, tetrahydrofuran and toluene, for procedures under inert atmosphere were prepared by distillation and drying over molecular sieve ( $3 \AA$ or $4 \AA$ A). Commercial materials and other solvents were purchased at the highest commercial quality from the providers Acros Organics, Alfa Aesar, Carbolution, Carl Roth, Merck, Sigma Aldrich, VWR, $\mathrm{TCl}$ Chemicals and Thermo Fisher Scientific. Air- and moisture-sensitive reactions were performed under nitrogen atmosphere using a Schlenk line. Before use, flasks were repeatedly evacuated (external heating) and refilled with nitrogen.

NMR: ${ }^{1} \mathrm{H}$ and ${ }^{13} \mathrm{C}$ Nuclear Magnetic Resonance Spectra (NMR) were recorded on Bruker AV300 and AV-600 spectrometers at $298 \mathrm{~K}$. The chemical shifts are given in $\delta$-values (ppm) and are calibrated on the residual peak of the deuterated solvent $\left(\mathrm{CDCl}_{3}: \delta_{\mathrm{H}}=7.26 \mathrm{ppm}, \delta_{\mathrm{C}}=77.0\right.$ ppm; DMSO-d $\left.\mathrm{d}_{6}: \delta_{H}=2.50 \mathrm{ppm}, \delta_{c}=39.5 \mathrm{ppm}, \mathrm{MeOD}-\mathrm{d}_{4}: \delta_{H}=3.31 \mathrm{ppm}, \delta_{\mathrm{c}}=49.0 \mathrm{ppm}\right)$. The coupling constants $J$ are given in Hertz $[\mathrm{Hz}]$. Following abbreviations were used for the allocation of signal multiplicities: bs - broad signal, s - singlet, $d$ - doublet, dd - doublet of doublets, ddd - doublet of doublets of doublets, $m$ - multiplet. DEPT spectra were calibrated on the first carbon signal.

MS: Elektrospray-Ionisation Mass spectra (ESI-MS) were recorded on an Advion expressionL CMS system using a single-quadrupole mass analyzer, a Peak Scientific N118LA nitrogen generator, an Edwards RV12 high vacuum pump and a Jasco PU-1580 Intelligent HPLC Pump, or a LCQ Fleet ion trap system (Thermo Scientific), which was combined with an UltiMate 300 HPLC system. For high resolution mass spectrometry (HRMS) an Agilent mass spectrometer 6538 with atmospheric pressure chemical ionization (APCI), high resolution Q-TOF mass analyzer and microchannel plate detector was used.

Chromatography: Thin-layer chromatography (TLC) was performed on precoated plates of silica gel F254 (Merck) with UV detection at 254 and $365 \mathrm{~nm}$. Column chromatography was

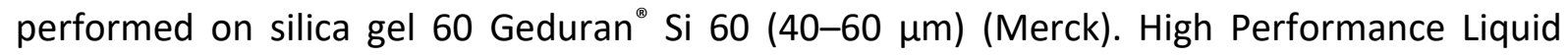
Chromatograms (HPLC) were recorded on a computer-controlled Jasco system including a UV1575 Intelligent UV/VIS Detector, DG-2080-53 3-Line Degaser, two PU-1580 Intelligent HPLC Pumps, AS-1550 Intelligent Sampler, HG-1580-32 Dynamic Mixer. A Eurospher II 100-3 C18 A $(150 \times 4.6 \mathrm{~mm})$ column with integrated precolumn manufactured by Knauer was used. The eluent system consisted of $\mathrm{A}=\mathrm{H}_{2} \mathrm{O}+0.05 \% \mathrm{TFA}, \mathrm{B}=\mathrm{MeCN}+0.05 \% \mathrm{TFA}$. The analytical method used the following elution gradient: 0-2 $\min (5 \%$ B), 2-25 min (linear increase to 95\% B), 25$28 \mathrm{~min}(95 \% \mathrm{~B}), 28-31 \mathrm{~min}(5 \% \mathrm{~B})$ with a flow of $1.0 \mathrm{~mL} / \mathrm{min}$. For medium pressure liquid chromatography (MPLC) the Reveleris ${ }^{\circledR}$ X MPLC system (Grace) was used together with Reverleris ${ }^{\oplus}$ Reverse Phase (RP) C18 columns (Grace) using UV-detection at $220 \mathrm{~nm}, 254 \mathrm{~nm}$, and $280 \mathrm{~nm}$. 


\section{Chemical Procedures}

\subsection{General Procedures}

\section{General Procedure for the Formation of lodonium Salts}

The iodonium salts were prepared according to a published procedure. ${ }^{1,2}$ The corresponding aryl iodide (1.0 eq.) was dissolved in dry acetonitrile $(3.0 \mathrm{~mL} / \mathrm{mmol}) . p$-Toluenesulfonic acid monohydrate (1.01 eq. for monoiodonium salt, 2.02 eq. for diiodonium salt) and mCPBA (1.1 eq. for monoiodonium salt, 2.2 eq. for diiodonium salt), were added. The reaction mixture was stirred at $55^{\circ} \mathrm{C}$ for $50 \mathrm{~min}$ before being treated with 1,3,5-trimethoxybenzene (1.01 eq. for monoiodonium salt, 2.02 eq. for diiodonium salt). The reaction was stirred at $55^{\circ} \mathrm{C}$ for another $15 \mathrm{~min}$ and concentrated under reduced pressure. The obtained crude residue was washed with $\mathrm{Et}_{2} \mathrm{O}(4 \mathrm{x})$ and dried in vacuo to give the desired product as a white solid which was used without further purification.

\section{General Procedure for the Nucleophilic Aromatic Substitution}

The diphenyl ether formation was conducted according to a published procedure. ${ }^{3} \mathrm{KO}^{\mathrm{t}} \mathrm{Bu}$ (1.1 eq. per OH-group) was dissolved in dry $\operatorname{THF}(4.5 \mathrm{~mL} / \mathrm{mmol})$ and cooled to $0{ }^{\circ} \mathrm{C}$. The corresponding alcohol (1.0 eq.) was added and the reaction solution was stirred for $15 \mathrm{~min}$ at $0{ }^{\circ} \mathrm{C}$. After addition of the iodonium salt (1.0 eq. per OH-Group), the reaction mixture was stirred at $40{ }^{\circ} \mathrm{C}$ for $1-64 \mathrm{~h}$. $\mathrm{H}_{2} \mathrm{O}$ was slowly added at $0{ }^{\circ} \mathrm{C}$ and the organic phase was separated. The aqueous layer was extracted with $\mathrm{Et}_{2} \mathrm{O}(2 \times)$. The combined organic layers were washed with brine, dried over $\mathrm{Na}_{2} \mathrm{SO}_{4}$ and filtered. The solvent was removed and the obtained residue was purified by MPLC to give the corresponding product or product mixtures.

\section{General Procedure for Suzuki Cross Coupling Reaction}

$\mathrm{Pd}_{2}(\mathrm{dba})_{3}(5 \mathrm{~mol} \%)$ and DavePhos (15 mol\%) were added in a Schlenk tube equipped with a stir bar. The Schlenk tube was evacuated and then refilled with nitrogen. Dry 1,4-dioxane $(5.00 \mathrm{~mL} / \mathrm{mmol})$ was added and three more evacuation-refill cycles (degassing) were performed. The catalyst mixture was heated to $90{ }^{\circ} \mathrm{C}$ using a preheated oil bath. After $20 \mathrm{~min}$, the aryl iodide (1.0 eq.), (3,5-Dichloro-2-methoxyphenyl)boronic acid (16, 2.0 eq.), potassium carbonate ( 2.0 eq.) and silver(I) oxide ( 0.5 eq.) were added and another three evacuation-refill cycles were performed. The reaction mixture was stirred at $90{ }^{\circ} \mathrm{C}$ for $24 \mathrm{~h}$ before being diluted with EtOAc at room temperature. The reaction solution was filtered, evaporated and the obtained residue was purified by MPLC to give the desired product as a yellowish solid.

\section{General Procedure for the Methoxy Deprotection}

The protected compound (1.0 eq.) was dissolved in dry DCM $(25 \mathrm{~mL} / \mathrm{mmol})$ and cooled to $0{ }^{\circ} \mathrm{C}$. $1 \mathrm{M}$ boron tribromide solution (15.0 eq.) was added dropwise and the reaction solution was stirred for $2 \mathrm{~h}$ at $0{ }^{\circ} \mathrm{C}$ and for $24-48 \mathrm{~h}$ at room temperature. The reaction was quenched with water at $0{ }^{\circ} \mathrm{C}$ and the organic phase was separated. The aqueous layer was extracted with DCM (2x). The combined organic layers were washed with brine, dried over $\mathrm{Na}_{2} \mathrm{SO}_{4}$ and filtered. The solvent was removed, and the obtained residue was purified by MPLC to give the corresponding deprotected phenols as white solids. 


\subsection{Synthesis of Ambigol A (1) and B (2)}

\section{3,5-Dichloro-2,6-diiodophenol (9)}

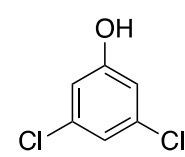

8

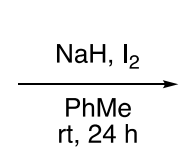

$\mathrm{rt}, 24 \mathrm{~h}$

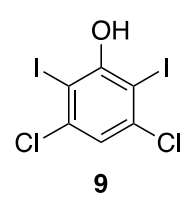

9

3,5-Dichlorophenol (8, $2.50 \mathrm{~g}, 15.3 \mathrm{mmol}, 1.0$ eq.) and sodium hydride (1.10 g, $45.9 \mathrm{mmol}$, 3.0 eq.) were slowly dissolved in dry toluene $(30.0 \mathrm{~mL}, 2.0 \mathrm{~mL} / \mathrm{mmol})$. lodine $(9.71 \mathrm{~g}$, $38.3 \mathrm{mmol}, 2.5$ eq.) was added and the obtained suspension was stirred for $24 \mathrm{~h}$ at room temperature. The reaction mixture was treated with $1 \mathrm{~N} \mathrm{NaOH}$ and the obtained layers were separated. The organic layer was extracted continuously with $1 \mathrm{~N} \mathrm{NaOH}$ and the combined aqueous layers were acidified with $4 \mathrm{~N} \mathrm{HCl}$. The resulting white precipitate was filtered off, washed with water and dried in vacuo. The crude product was purified by column chromatography ( $\mathrm{n}$-pentane/EtOAc $=10: 1)$ to give the desired product $(3.57 \mathrm{~g}, 8.61 \mathrm{mmol}$, $56 \%)$ as a white solid.

${ }^{1} \mathrm{H}-\mathrm{NMR}\left(300 \mathrm{MHz}, \mathrm{CDCl}_{3}\right): \delta=7.23(\mathrm{~s}, 1 \mathrm{H}), 6.17(\mathrm{~s}, 1 \mathrm{H}) .{ }^{13} \mathrm{C}-\mathrm{NMR}\left(75 \mathrm{MHz}, \mathrm{CDCl}_{3}\right): \delta=156.7$, 139.8, 121.3, 85.4. MS (ESI+): $m / z=414.4[\mathrm{M}+\mathrm{H}]^{+}$. HRMS (ESI-TOF) $m / z$ : $[\mathrm{M}-\mathrm{H}]^{-}$calcd for $\mathrm{C}_{6} \mathrm{HCl}_{2} \mathrm{I}_{2} \mathrm{O} 412.7499$ (100\%), 414.7470 (64\%), 416.7440 (10\%), 413.7533 (7\%); found 412.7490 (100\%), 414.7464 (61\%), 416.7430 (8\%), 413.7518 (5\%). TLC: $R_{f}$ (n-pentane/EtOAC = $8: 1)=0.67$.

\section{1,5-Dichloro-2,4-diiodo-3-methoxybenzene (10)}

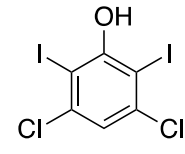

9

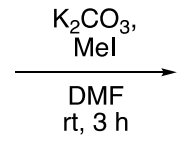

10

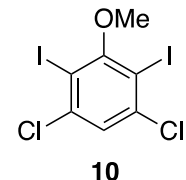

3,5-Dichloro-2,6-diiodophenol (9, $3.00 \mathrm{~g}, 7.23 \mathrm{mmol}, 1.0$ eq.) was dissolved in DMF (7.20 mL, $1.0 \mathrm{~mL} / \mathrm{mmol})$ and potassium carbonate $(1.20 \mathrm{~g}, 8.68 \mathrm{mmol}, 1.2 \mathrm{eq}$.$) was added. After$ dropwise addition of methyl iodide $(0.54 \mathrm{~mL}, 1.23 \mathrm{~g}, 8.68 \mathrm{mmol}, 1.2 \mathrm{eq}$.$) , the reaction solution$ was stirred for $3 \mathrm{~h}$ at room temperature. After completion, the reaction was diluted with water and $\mathrm{Et}_{2} \mathrm{O}$. The aqueous layer was extracted with $\mathrm{Et}_{2} \mathrm{O}(2 \times)$. The combined organic layers were washed with $1 \mathrm{~N} \mathrm{HCl}(6 \times)$, brine, dried over $\mathrm{Na}_{2} \mathrm{SO}_{4}$ and filtered. The solvent was removed to give 1,5-dichloro-2,4-diiodo-3-methoxybenzene (3.02 g, $7.04 \mathrm{mmol}, 97 \%$ ) as colorless solid.

${ }^{1} \mathrm{H}-\mathrm{NMR}\left(300 \mathrm{MHz}, \mathrm{CDCl}_{3}\right): \delta=7.40(\mathrm{~s}, 1 \mathrm{H}), 3.87(\mathrm{~s}, 3 \mathrm{H}) \cdot{ }^{13} \mathrm{C}-\mathrm{NMR}\left(75 \mathrm{MHz}, \mathrm{CDCl}_{3}\right): \delta=162.4$, 140.4, 125.2, 94.2, 60.7. MS (ESI+): $\mathrm{m} / \mathrm{z}=428.8[\mathrm{M}+\mathrm{H}]^{+}$. HRMS (APCI-TOF) $\mathrm{m} / \mathrm{z}:[\mathrm{M}]^{+}$calcd for $\mathrm{C}_{7} \mathrm{H}_{4} \mathrm{Cl}_{2} \mathrm{I}_{2} \mathrm{O} 427.7723$ (100\%), 429.7694 (64\%), 431.7664 (10\%), 428.7757 (8\%), 430.7727 (5\%); found 427.7718 (100\%), 429.7686 (56\%), 431.7660 (9\%), 428.7751 (6\%), 430.7725 (5\%). TLC: $R_{f}(n-p e n t a n e / E t O A c=20: 1)=0.85$. 
(4,6-Dichloro-2-methoxy-1,3-phenylene)bis((2,4,6-trimethoxyphenyl)iodonium) Bistosylat (12)

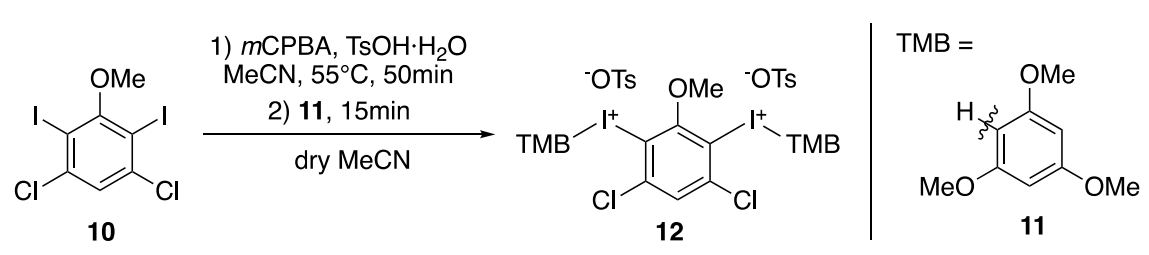

The diiodonium salt was prepared according to the general procedure in section 2.1. The salt was synthesized in $80 \%$ yield $(1.44 \mathrm{~g}, 1.30 \mathrm{mmol})$ as a white solid.

${ }^{1} \mathrm{H}-\mathrm{NMR}\left(600 \mathrm{MHz}, \mathrm{DMSO}-\mathrm{d}_{6}\right): \delta=7.93(\mathrm{~s}, 1 \mathrm{H}), 7.46(\mathrm{~d}, J=8.1 \mathrm{~Hz}, 4 \mathrm{H}), 7.11(\mathrm{~d}, J=8.1 \mathrm{~Hz}, 4 \mathrm{H})$, $6.42(\mathrm{~s}, 2 \mathrm{H}), 6.28(\mathrm{~s}, 2 \mathrm{H}), 3.92(\mathrm{~s}, 3 \mathrm{H}), 3.90(\mathrm{~s}, 6 \mathrm{H}), 3.85(\mathrm{~s}, 3 \mathrm{H}), 3.84(\mathrm{~s}, 3 \mathrm{H}), 3.76(\mathrm{~s}, 6 \mathrm{H})$, $2.28(\mathrm{~s}, 6 \mathrm{H}) . \mathrm{MS}(\mathrm{ESI}+): \mathrm{m} / \mathrm{z}=932.8[\mathrm{M}-\mathrm{OTs}]^{+}, 761.5[\mathrm{M}-2 \mathrm{OTs}]^{2+}$. HRMS (ESI-TOF) $\mathrm{m} / \mathrm{z}$ : $[\mathrm{M}+\mathrm{Na}]^{+}$cald for $\mathrm{C}_{39} \mathrm{H}_{40} \mathrm{Cl}_{2} \mathrm{I}_{2} \mathrm{NaO}_{13} \mathrm{~S}_{2} 1126.9269$ (100\%), 1128.9239 (64\%), 1127.9303 (42\%), 1129.9273 (27\%), 1130.9210 (10\%); found 1126.9250 (100\%), $1128.9236(83 \%), 1127.9278$ (48\%), 1129.9257 (33\%), 1130.9233 (24\%). TLC: $R_{f}(D C M / M e O H=3: 1)=0.47$.

\section{Nucleophilic Aromatic Substitution of 2,4-Dichlorophenol (13) with Compound 12}<smiles>Oc1ccc(Cl)cc1Cl</smiles>

13

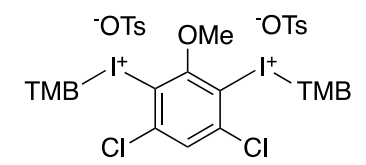

12

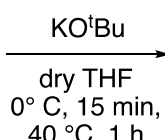
$40^{\circ} \mathrm{C}, 1 \mathrm{~h}$ $40^{\circ} \mathrm{C}, 1 \mathrm{~h}$<smiles>COc1c(I)c(Cl)cc(Cl)c1Oc1ccc(Cl)cc1Cl</smiles>

14<smiles>COc1c(Oc2ccc(Cl)cc2Cl)c(Cl)cc(Cl)c1Oc1ccc(Cl)cc1Cl</smiles>

15

The nucleophilic aromatic substitution was performed according to the general procedure in section 2.1. 1,5-Dichloro-2-(2,4-dichlorophenoxy)-4-iodo-3-methoxybenzene (14) was synthesized in $65 \%$ yield $(272 \mathrm{mg}, 0.59 \mathrm{mmol})$ and 4,4 - $-((4,6$-dichloro-2-methoxy-1,3phenylene)bis(oxy))bis(1,3-di-chlorobenzene) (15) in $27 \%$ yield (122 $\mathrm{mg}, 0.24 \mathrm{mmol}$ ). Therefore, the reaction was performed with a total iodononium salt conversion of $92 \%$. Both compounds were obtained as yellowish solids.

\section{1,5-Dichloro-2-(2,4-dichlorophenoxy)-4-iodo-3-methoxybenzene (14)}<smiles>COc1c(Cl)cc(Cl)c(I)c1Oc1ccc(Cl)cc1Cl</smiles>

14

${ }^{1} \mathrm{H}-\mathrm{NMR}\left(300 \mathrm{MHz}, \mathrm{CDCl}_{3}\right): \delta=7.46(\mathrm{~d}, J=2.5 \mathrm{~Hz}, 1 \mathrm{H}), 7.44(\mathrm{~s}, 1 \mathrm{H}), 7.08(\mathrm{dd}, J=8.8,2.5 \mathrm{~Hz}$, $1 \mathrm{H}), 6.40(\mathrm{~d}, J=8.8 \mathrm{~Hz}, 1 \mathrm{H}), 3.86(\mathrm{~s}, 3 \mathrm{H}) .{ }^{13} \mathrm{C}-\mathrm{NMR}\left(75 \mathrm{MHz}, \mathrm{CDCl}_{3}\right): \delta=155.2,151.3,142.0$, 136.6, 130.6, 129.8, 128.2, 127.9, 125.8, 123.3, 115.4, 96.7, 61.7. HRMS (APCI-TOF) $\mathrm{m} / \mathrm{z}$ : [M$\mathrm{H}]^{-}$calcd for $\mathrm{C}_{13} \mathrm{H}_{6} \mathrm{Cl}_{4} \mathrm{IO}_{2} 462.8143$ (100\%), 460.8172 (78\%), 464.8113 (48\%), 463.8176 (14\%); found 462.8125 (100\%), 460.8119 (76\%), 464.8159 (59\%), 463.8155 (14\%). TLC: $R_{f}(n-$ pentane $/$ EtOAc $=20: 1)=0.83$. 


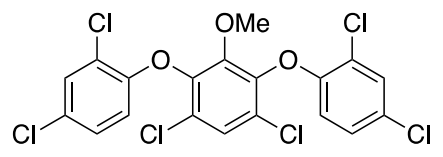

15

${ }^{1} \mathrm{H}-\mathrm{NMR}\left(300 \mathrm{MHz}, \mathrm{CDCl}_{3}\right): \delta=7.45$ (d, $\left.J=2.5 \mathrm{~Hz}, 2 \mathrm{H}\right), 7.40(\mathrm{~s}, 1 \mathrm{H}), 7.10$ (dd, J = 8.8, $2.5 \mathrm{~Hz}$, $2 \mathrm{H}$ ), 6.40 (d, $J=8.8 \mathrm{~Hz}, 2 \mathrm{H}$ ), $3.72(\mathrm{~s}, 3 \mathrm{H}) .{ }^{13} \mathrm{C}-\mathrm{NMR}$ (75 MHz, CDCl $): \delta=151.7,148.0,143.9$, 130.7, 128.3, 127.8, 126.2, 125.5, 123.6, 115.6, 62.1. HRMS (APCl-TOF) $\mathrm{m} / \mathrm{z}$ : [M] ${ }^{+}$calcd for $\mathrm{C}_{19} \mathrm{H}_{10} \mathrm{Cl}_{6} \mathrm{IO}_{3} 497.8732$ (100\%), 499.8702 (80\%), 495.8761 (52\%), 501.8673 (34\%), 498.8765 (17\%); found 497.8717 (100\%), 499.8692 (81\%), 495.8750 (51\%), 501.8659 (44\%), 498.8788 (27\%). TLC: $R_{f}(n-p e n t a n e / E t O A c=20: 1)=0.77$.

\section{3',4,5',6-Tetrachloro-3-(2,4-dichlorophenoxy)-2,2'-dimethoxy-1,1'-biphenyl (17)}<smiles>COc1c(I)c(Cl)cc(Cl)c1Oc1ccc(Cl)cc1Cl</smiles>

14<smiles>COc1c([18OH])cc(Cl)cc1Cl</smiles>

16

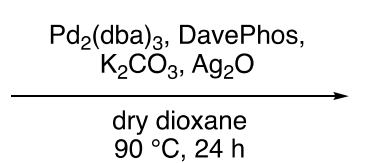
$90^{\circ} \mathrm{C}, 24 \mathrm{~h}$<smiles>COc1c(Cl)cc(Cl)cc1-c1c(Cl)cc(Cl)c(Oc2ccc(Cl)cc2Cl)c1OC</smiles>

17

The Suzuki coupling was performed according to the general procedure in section 2.1. The product was obtained in $41 \%$ yield $(90.0 \mathrm{mg}, 0.18 \mathrm{mmol})$ as a yellowish solid. The substrate 1,5-dichloro-2-(2,4-dichlorophenoxy)-4-iodo-3-methoxybenzene $(53.0 \mathrm{mg}, 0.11 \mathrm{mmol}$ ) was reisolated in $27 \%$ yield, based on which the desired product was obtained in $55 \%$ isolated yield.

${ }^{1} \mathrm{H}-\mathrm{NMR}\left(300 \mathrm{MHz}, \mathrm{CDCl}_{3}\right): \delta=7.46(\mathrm{~d}, J=2.5 \mathrm{~Hz}, 1 \mathrm{H}), 7.45(\mathrm{~d}, J=2.5 \mathrm{~Hz}, 1 \mathrm{H}), 7.42(\mathrm{~s}, 1 \mathrm{H})$, 7.13-7.06 (m, $2 \mathrm{H}), 6.46$ (dd, J = 8.8, $2.5 \mathrm{~Hz}, 1 \mathrm{H}), 3.68$ (s, $3 \mathrm{H}), 3.63(\mathrm{~s}, 3 \mathrm{H}) .{ }^{13} \mathrm{C}-\mathrm{NMR}(75 \mathrm{MHz}$, $\left.\mathrm{CDCl}_{3}\right): \delta=152.9,152.6,151.4,142.6,131.3,131.2,130.8,130.6,130.4,129.6,129.3,129.1$, $128.1,127.9,125.9,123.3,115.1,62.0,61.1$. One carbon signal missing due to overlap. HRMS (ESI-TOF) $m / z$ : $[\mathrm{M}+\mathrm{Na}]^{+}$calcd for $\mathrm{C}_{20} \mathrm{H}_{12} \mathrm{Cl}_{6} \mathrm{NaO}_{3} 534.8780$ (100\%), 536.8751 (80\%), 532.8810 (52\%), 538.8721 (34\%), 535.8814 (18\%); found 534.8779 (100\%), 536.8738 (93\%), 532.8828 (57\%), 538.8715 (35\%), $535.8797(23 \%)$. TLC: $R_{f}(n-p e n t a n e / E t O A c=20: 1)=0.92$.

3',4,5',6-tetrachloro-3-(2,4-dichlorophenoxy)-[1,1'-biphenyl]-2,2'-diol - ambigol A (1)<smiles>COc1c(Cl)cc(Cl)cc1-c1c(Cl)cc(Cl)c(Oc2ccc(Cl)cc2Cl)c1OC</smiles>

17

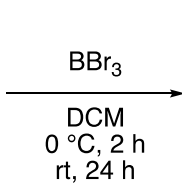

$\mathrm{rt}, 24 \mathrm{~h}$<smiles>Oc1c(Cl)cc(Cl)cc1-c1c(Cl)cc(Cl)c(Oc2ccc(Cl)cc2Cl)c1O</smiles>

1

The reaction was carried out analogous to the general procedure in section 2.1. The corresponding deprotected diphenol 1 was synthesized in $95 \%$ yield $(78.0 \mathrm{mg}, 0.16 \mathrm{mmol})$ as a white solid. Analytical data were in agreement with literature values. ${ }^{4}$

${ }^{1} \mathrm{H}-\mathrm{NMR}\left(300 \mathrm{MHz}, \mathrm{CDCl}_{3}\right): \delta=7.46(\mathrm{~d}, J=2.5 \mathrm{~Hz}, 1 \mathrm{H}), 7.42(\mathrm{~d}, J=2.5 \mathrm{~Hz}, 1 \mathrm{H}), 7.21(\mathrm{~s}, 1 \mathrm{H})$, 7.15-7.09 (m, $2 \mathrm{H}), 6.60$ (dd, $J=8.8,2.5 \mathrm{~Hz}, 1 \mathrm{H}$ ), 4.97 (bs, $2 \mathrm{H}) .{ }^{13} \mathrm{C}-\mathrm{NMR}\left(75 \mathrm{MHz}, \mathrm{CDCl}_{3}\right.$ ): $\delta$ $=151.0,148.7,148.2,137.4,131.9,130.6,130.4,129.4,128.7,128.2,128.0,125.5,123.8$, 
122.7, 122.6, 122.5, 121.5, 115.9. ${ }^{1} \mathrm{H}-\mathrm{NMR}\left(300 \mathrm{MHz}, \mathrm{MeOD}-\mathrm{d}_{4}\right): \delta=7.51(\mathrm{~d}, J=2.6 \mathrm{~Hz}, 1 \mathrm{H})$, $7.41(\mathrm{~d}, J=2.6 \mathrm{~Hz}, 1 \mathrm{H}), 7.19(\mathrm{~s}, 1 \mathrm{H}), 7.18(\mathrm{dd}, J=8.8,2.6 \mathrm{~Hz}, 1 \mathrm{H}), 7.08(\mathrm{~d}, J=2.6 \mathrm{~Hz}, 1 \mathrm{H}), 6.99$ (d, $J=8.9 \mathrm{~Hz}, 1 \mathrm{H}$ ). ${ }^{13} \mathrm{C}-\mathrm{NMR}\left(75 \mathrm{MHz}, \mathrm{MeOD}-\mathrm{d}_{4}\right): \delta=153.2,151.9,151.3,139.1,132.9,131.1$, 131.0, 130.2, 128.91, 128.85, 128.6, 126.3, 125.7, 125.2, 124.8, 123.4, 122.0, 117.0. MS (ESI): $m / z=480.8[\mathrm{M}-\mathrm{H}]^{-}$. HRMS (ESI-TOF) $\mathrm{m} / z$ : $[\mathrm{M}-\mathrm{H}]^{-}$calcd for $\mathrm{C}_{18} \mathrm{H}_{7} \mathrm{Cl}_{6} \mathrm{O}_{3} 482.8502$ (100\%), 480.8532 (78\%), 484.8473 (72\%); found 482.8489 (100\%), 480.8521 (51\%), 484.8464 (78\%). HRMS (ESI+) $m / z$ calcd for $\mathrm{C}_{18} \mathrm{H}_{7} \mathrm{Cl}_{6} \mathrm{NaO}_{3}[\mathrm{M}+\mathrm{Na}]^{+}: 506.8467$ (100\%), 504.8497 (78\%), 508.8438 (72\%); found: 506.8458 (100\%), 504.8490 (55\%), 508.8429 (82\%). TLC: $R_{f}$ (n-pentane/EtOAc = $1: 1)=0.73$. HPLC: $t_{R}=18.85 \mathrm{~min}$.

3,5-Dichloro-2,6-bis(2,4-dichlorophenoxy)phenol - ambigol B (2)<smiles>COc1c(Cl)cc(Cl)c(Oc2ccc(Cl)cc2Cl)c1Oc1ccc(Cl)cc1Cl</smiles>

15

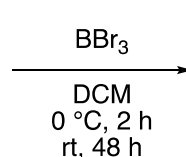

rt, $48 \mathrm{~h}$<smiles>Oc1c(Oc2ccc(Cl)cc2Cl)c(Cl)cc(Cl)c1Oc1ccc(Cl)cc1Cl</smiles>

2

Compound $\mathbf{2}$ was synthesized following the general procedure in section 2.1 and isolated as a white solid $(28.0 \mathrm{mg}, 57.7 \mu \mathrm{mol}, 76 \%)$. Analytical data were in agreement with literature values. ${ }^{4}$

${ }^{1} \mathrm{H}-\mathrm{NMR}\left(300 \mathrm{MHz}, \mathrm{CDCl}_{3}\right): \delta=7.47(\mathrm{~d}, J=2.5 \mathrm{~Hz}, 2 \mathrm{H}), 7.19(\mathrm{~s}, 1 \mathrm{H}), 7.12$ (dd, J= 8.8, $2.5 \mathrm{~Hz}$, $2 \mathrm{H}), 6.56$ (d, J = 8.8 Hz, $2 \mathrm{H}$ ), 5.52 (bs, $1 \mathrm{H}) .{ }^{13} \mathrm{C}-\mathrm{NMR}\left(75 \mathrm{MHz}, \mathrm{CDCl}_{3}\right): \delta=151.1,144.4,138.2$, 130.8, 128.8, 127.9, 125.8, 123.9, 122.0, 115.8. ${ }^{1} \mathrm{H}-\mathrm{NMR}(300 \mathrm{MHz}, \mathrm{MeOD}): \delta=7.52$ (d, $J=2.5 \mathrm{~Hz}, 2 \mathrm{H}$ ), $7.24(\mathrm{~s}, 1 \mathrm{H}), 7.20$ (dd, J = 8.9, $2.5 \mathrm{~Hz}, 2 \mathrm{H}), 6.65(\mathrm{~d}, J=8.9 \mathrm{~Hz}, 2 \mathrm{H}) .{ }^{13} \mathrm{C}-\mathrm{NMR}$ (75 MHz, MeOD): $\delta=153.0,147.6,140.2,131.2,128.9,128.8,126.6,124.6,121.6,116.8 .{ }^{1} \mathrm{H}-$ NMR $(300 \mathrm{MHz}, \mathrm{MeOD}+$ drop of $1 \mathrm{~N} \mathrm{NaOH}): \delta=7.43(\mathrm{~d}, J=2.2 \mathrm{~Hz}, 2 \mathrm{H}), 7.12$ (dd, J=8.7, $2.6 \mathrm{~Hz}, 2 \mathrm{H}), 6.85-6.66(\mathrm{~m}, 1 \mathrm{H}), 6.62(\mathrm{~d}, J=8.9 \mathrm{~Hz}, 2 \mathrm{H}) . \mathbf{M S}(\mathrm{ESI}-): \mathrm{m} / z=480.8[\mathrm{M}-\mathrm{H}]^{-}$. HRMS (ESI-TOF) $m / z$ : [M-H] $]^{-}$calcd for $\mathrm{C}_{18} \mathrm{H}_{7} \mathrm{Cl}_{6} \mathrm{O}_{3} 482.8502$ (100\%), 480.8532 (78\%), 484.8473 (72\%), 484.8473 (48\%); found 482.8489 (100\%), 480.8519 (50\%), 484.8462 (79\%). TLC: $R_{f}(n-$ pentane $/$ EtOAC $=1: 1)=0.86$. HPLC: $\mathrm{t}_{\mathrm{R}}=19.55 \mathrm{~min}$. 


\subsection{Screening for Suzuki Coupling Conditions}<smiles>COc1c(Cl)cc(Cl)cc1I</smiles>

18<smiles>COc1c(O)cc(Cl)cc1Cl</smiles>

16

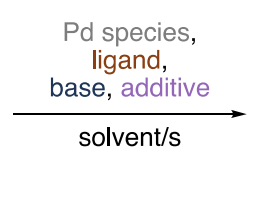
se, additive solvent/s

$\rightarrow \mathrm{Cl}$<smiles>COc1c(Cl)cc(Cl)cc1-c1cc(Cl)cc(Cl)c1OC</smiles>

19
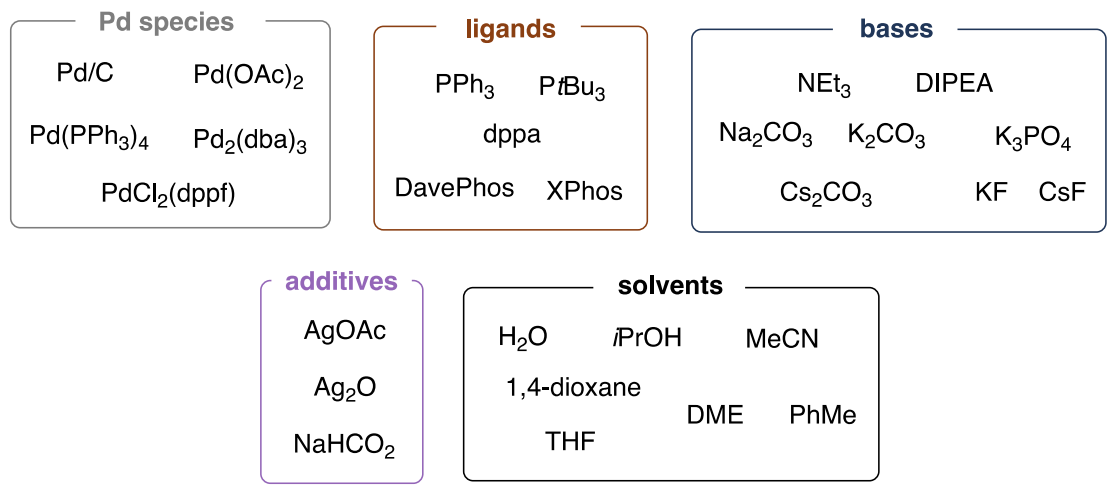

lodo aryl 18 and boronic acid $\mathbf{1 6}$ were prepared according to published procedures. ${ }^{5,6}$

Exemplary procedure for Suzuki test reaction (Table S1, entry 18): $\mathrm{PdCl}_{2}$ (dppf) (13.2 mg, $0.02 \mathrm{mmol}, 6.0 \mathrm{~mol} \%)$ was filled in a Schlenk tube equipped with a stir bar The Schlenk tube was evacuated and then refilled with nitrogen. Dry 1,4-dioxane $(1.50 \mathrm{~mL}, 5.00 \mathrm{~mL} / \mathrm{mmol})$ was added and three more evacuation-refill cycles (degassing) were performed. After short stirring, the aryl iodide $18(86.7 \mathrm{mg}, 0.20 \mathrm{mmol}, 1.0$ eq.), (3,5-dichloro-2methoxyphenyl)boronic acid $(16,66.3 \mathrm{mg}, 0.30 \mathrm{mmol}, 1.0$ eq.), potassium carbonate ( $82.9 \mathrm{mg}, 0.60 \mathrm{mmol}, 2.0$ eq.) and silver(I) acetate $(25.0 \mathrm{mg}, 0.15 \mathrm{mmol}, 0.5$ eq.) were added and another three evacuation-refill cycles were performed. The reaction mixture was stirred at $90^{\circ} \mathrm{C}$ for $24 \mathrm{~h}$ before being diluted with EtOAc at room temperature. The reaction solution was filtered and analyzed by HPLC. 


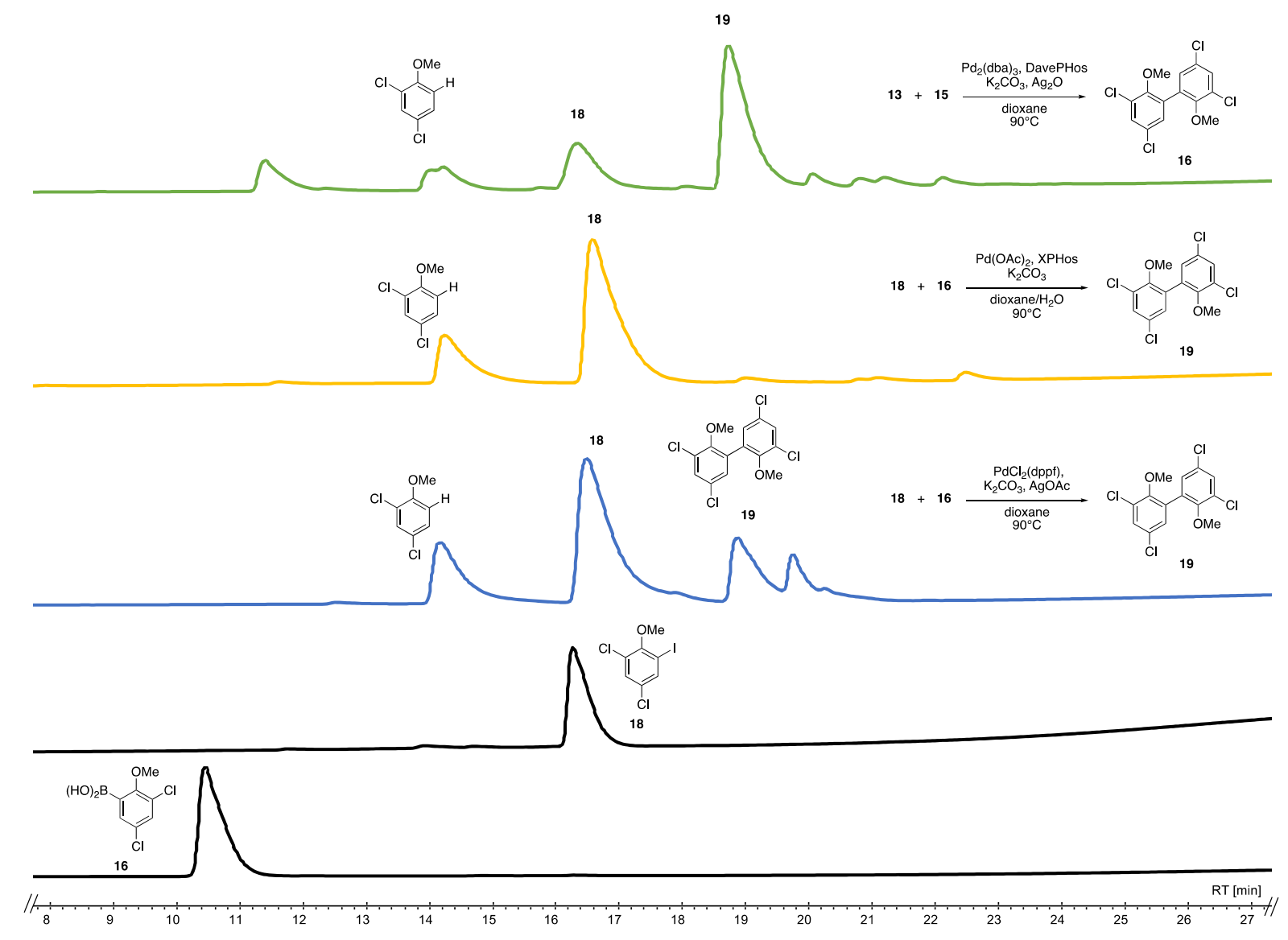

Fig. S1 HPLC analysis (after $24 \mathrm{~h}$ ) of three selected examples using different catalyst system. 


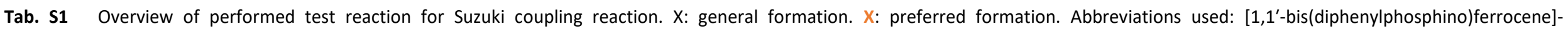

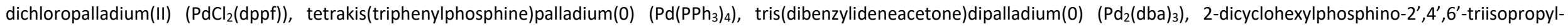
biphenyl (XPhos), bis(diphenylphosphino)acetylene (dppa), 2-dicyclohexylphosphino-2'-( $N, N$-dimethylamino)biphenyl (DavePhos), $N, N$-diisopropylethylamine (DIPEA), microwave (MW).

\begin{tabular}{|c|c|c|c|c|c|c|c|c|c|}
\hline entry & $\begin{array}{l}\text { catalyst } \\
\text { (mol\%) }\end{array}$ & $\begin{array}{l}\text { ligand } \\
\text { (mol\%) }\end{array}$ & $\begin{array}{l}\text { base } \\
\text { (eq.) }\end{array}$ & $\begin{array}{l}\text { additive } \\
\text { (eq.) }\end{array}$ & solvent/s & condition & 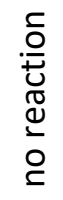 & 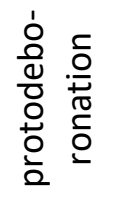 & 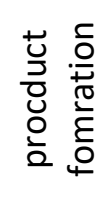 \\
\hline $1^{7}$ & $\mathrm{Pd} / \mathrm{C}(2.0,10.0)$ & & $\mathrm{K}_{2} \mathrm{CO}_{3}(4.0)$ & & $\mathrm{H}_{2} \mathrm{O}$ & $80^{\circ} \mathrm{C}$ & & $X$ & \\
\hline $2^{8}$ & $\mathrm{Pd} / \mathrm{C}(4.0)$ & & $\mathrm{K}_{3} \mathrm{PO}_{4}(3.5)$ & & $\mathrm{iPrOH} / \mathrm{H}_{2} \mathrm{O}=1: 1$ & $80^{\circ} \mathrm{C}$ & $X$ & & \\
\hline 3 & $\mathrm{Pd} / \mathrm{C}(4.0)$ & & KF (4.0) & & $\mathrm{H}_{2} \mathrm{O}$ & $80^{\circ} \mathrm{C}$ & $x$ & & \\
\hline 4 & $\mathrm{Pd}(\mathrm{OAc})_{2}(1.0)$ & $\mathrm{PPh}_{3}(2.0)$ & KF (3.3) & & THF & $\mathrm{rt}$ & $x$ & & \\
\hline 5 & $\mathrm{Pd}(\mathrm{OAc})_{2}(3.0)$ & XPhos (6.0) & $\mathrm{K}_{2} \mathrm{CO}_{3}(3.0)$ & & dioxane $/ \mathrm{H}_{2} \mathrm{O}=10: 1$ & $90^{\circ} \mathrm{C}$ & & $x$ & \\
\hline 6 & $\mathrm{Pd}(\mathrm{OAc})_{2}(4.0)$ & & $\mathrm{Na}_{2} \mathrm{CO}_{3}(1.5)$ & TBAI (0.25) & $\mathrm{H}_{2} \mathrm{O}$ & $130{ }^{\circ} \mathrm{C}$ & & $x$ & \\
\hline $7^{9}$ & $\mathrm{Pd}(\mathrm{OAc})_{2}(5.0)$ & $\mathrm{PPh}_{3}(10.0)$ & $\mathrm{K}_{3} \mathrm{PO}_{4}(2.0)$ & & $\mathrm{MeCN} / \mathrm{H}_{2} \mathrm{O}=4: 1$ & $50^{\circ} \mathrm{C}$ & $X$ & & \\
\hline 8 & $\mathrm{Pd}(\mathrm{OAc})_{2}(5.0)$ & $\mathrm{PPh}_{3}(10.0)$ & $\mathrm{K}_{2} \mathrm{CO}_{3}(4.0)$ & & $\mathrm{DME} / \mathrm{H}_{2} \mathrm{O}=3: 1$ & $80^{\circ} \mathrm{C}$ & & $x$ & \\
\hline 9 & $\mathrm{PdCl}_{2}(\mathrm{dppf})(3.0)$ & & $\mathrm{K}_{2} \mathrm{CO}_{3}(2.0)$ & & $\mathrm{THF} / \mathrm{H}_{2} \mathrm{O}=10: 1$ & $60{ }^{\circ} \mathrm{C}$ & $X$ & & \\
\hline 10 & $\mathrm{PdCl}_{2}$ (dppf) (3.0) & & KF (3.3) & & dioxane & $90^{\circ} \mathrm{C}$ & & $x$ & \\
\hline 11 & $\mathrm{PdCl}_{2}(\mathrm{dppf})(4.0)$ & & DIPEA (3.0) & & $\mathrm{iPrOH} / \mathrm{H}_{2} \mathrm{O}=2: 1$ & $100{ }^{\circ} \mathrm{C}(\mathrm{MW})$ & & $x$ & \\
\hline 12 & $\mathrm{PdCl}_{2}(\mathrm{dppf})(8.0)$ & & $\mathrm{Cs}_{2} \mathrm{CO}_{3}(3.0)$ & & THF & $55^{\circ} \mathrm{C}$ & & $x$ & \\
\hline 13 & $\mathrm{PdCl}_{2}(\mathrm{dppf})(8.0)$ & & $\mathrm{Na}_{2} \mathrm{CO}_{3}(2.5)$ & & dioxane & $80^{\circ} \mathrm{C}$ & & $x$ & \\
\hline 14 & $\mathrm{PdCl}_{2}(\mathrm{dppf})(8.0)$ & & $\mathrm{NEt}_{3}(3.0)$ & & dioxane & $90^{\circ} \mathrm{C}$ & & $x$ & $x$ \\
\hline 15 & $\mathrm{PdCl}_{2}(\mathrm{dppf})(8.0)$ & & $\operatorname{CsF}(3.0)$ & & dioxane & $90^{\circ} \mathrm{C}$ & & $x$ & $x$ \\
\hline 16 & $\mathrm{PdCl}_{2}(\mathrm{dppf})(8.0)$ & & $\mathrm{Na}_{2} \mathrm{CO}_{3}(2.5)$ & $\mathrm{NaHCO}_{2}(0.5)$ & dioxane & $90^{\circ} \mathrm{C}$ & & $x$ & \\
\hline 17 & $\mathrm{PdCl}_{2}(\mathrm{dppf})(8.0)$ & & $\mathrm{K}_{2} \mathrm{CO}_{3}(2.0)$ & $\mathrm{Ag}_{2} \mathrm{O}(0.5)$ & dioxane & $90{ }^{\circ} \mathrm{C}$ & & $x$ & $x$ \\
\hline 18 & $\mathrm{PdCl}_{2}(\mathrm{dppf})(8.0)$ & & $\mathrm{K}_{2} \mathrm{CO}_{3}(2.0)$ & $\mathrm{AgOAc}(0.5)$ & dioxane & $90^{\circ} \mathrm{C}$ & & $x$ & $x$ \\
\hline 19 & $\mathrm{Pd}\left(\mathrm{PPh}_{3}\right)_{4}(3.0)$ & & $\mathrm{Na}_{2} \mathrm{CO}_{3}(2.5)$ & & PhMe & rt & $X$ & & \\
\hline $20^{10}$ & $\mathrm{Pd}\left(\mathrm{PPh}_{3}\right)_{4}(10.0)$ & & $\mathrm{Na}_{2} \mathrm{CO}_{3}(1.4)$ & & $\mathrm{PhMe} / \mathrm{EtOH}=1: 1$ & $60^{\circ} \mathrm{C}$ & & $x$ & \\
\hline 21 & $\mathrm{Pd}_{2}(\mathrm{dba})_{3}(1.0)$ & $\mathrm{P}(t \mathrm{Bu})_{3}(2.0)$ & KF (3.3) & & dioxane & $90^{\circ} \mathrm{C}$ & & $x$ & \\
\hline 22 & $\mathrm{Pd}_{2}(\mathrm{dba})_{3}(5.0)$ & dppa (15.0) & $\mathrm{K}_{2} \mathrm{CO}_{3}(2.0)$ & $\mathrm{Ag}_{2} \mathrm{O}(0.5)$ & dioxane & $90^{\circ} \mathrm{C}$ & & $x$ & $x$ \\
\hline 23 & $\mathrm{Pd}_{2}(\mathrm{dba})_{3}(5.0)$ & DavePhos (15.0) & $\mathrm{K}_{2} \mathrm{CO}_{3}(2.0)$ & $\mathrm{Ag}_{2} \mathrm{O}(0.5)$ & dioxane & $90{ }^{\circ} \mathrm{C}$ & & $x$ & $x$ \\
\hline
\end{tabular}




\subsection{Synthesis of Ambigol C (3) and Derivative 30}

\section{(4,6-Dichloro-5-methoxy-1,3-phenylene)bis((2,4,6-trimethoxyphenyl)iodonium) Bistosylat}

(22)

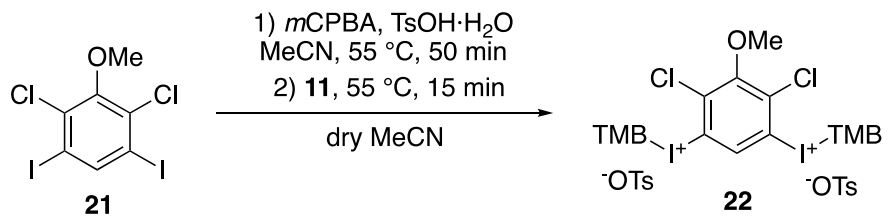

The diiodonium salt was prepared according to the general procedure in section 2.1. The salt was synthesized in 73\% (3.39 g, $3.07 \mathrm{mmol}$ ) yield as a beige solid.

${ }^{1}$ H-NMR $\left(300 \mathrm{MHz}, \mathrm{DMSO}-\mathrm{d}_{6}\right): \delta=8.46(\mathrm{~s}, 1 \mathrm{H}), 7.45(\mathrm{~d}, J=8.0 \mathrm{~Hz}, 4 \mathrm{H}), 7.45(\mathrm{~d}, J=8.0 \mathrm{~Hz}, 4 \mathrm{H})$, 6.46-6.45 (m, $4 \mathrm{H}), 3.94(\mathrm{~s}, 6 \mathrm{H}), 3.85(\mathrm{~s}, 6 \mathrm{H}), 3.80(\mathrm{~s}, 6 \mathrm{H}), 3.75(\mathrm{~s}, 3 \mathrm{H}), 2.28(\mathrm{~s}, 6 \mathrm{H}) . \mathrm{MS}(\mathrm{ESI}+)$ : $\mathrm{m} / \mathrm{z}=933.4[\mathrm{M}-\mathrm{OTs}]^{+}, 761.9[\mathrm{M}-2 \mathrm{OTs}]^{2+} . \mathrm{MS}$ (ESI-): $\mathrm{m} / \mathrm{z}=1102.7[\mathrm{M}-\mathrm{H}]^{-}$. HRMS (ESI-TOF) $\mathrm{m} / \mathrm{z}$ : $[\mathrm{M}+\mathrm{Na}]^{+}$cald for $\mathrm{C}_{39} \mathrm{H}_{40} \mathrm{Cl}_{2} \mathrm{I}_{2} \mathrm{NaO}_{13} \mathrm{~S}_{2} 1126.9269$ (100\%), 1128.9239 (64\%), 1127.9303 (42\%), 1129.9273 (27\%), 1130.9210 (10\%), found $1126.9233(100 \%), 128.9218$ (85\%), 1127.9263 (48\%), 1129.9243 (37\%), 1130.9212 (24\%).

\section{Nucleophilic Aromatic Substitution of 2,4-Dichlorophenol with Compound 22}<smiles>Oc1ccc(Cl)cc1Cl</smiles>

13

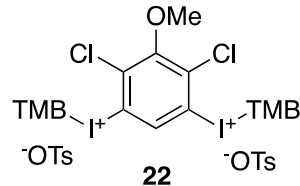

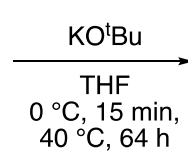

$40^{\circ} \mathrm{C}, 64 \mathrm{~h}$<smiles>COc1c(Cl)c(I)cc(Oc2ccc(Cl)cc2Cl)c1Cl</smiles>

23<smiles>COc1c(Cl)c(Oc2ccc(Cl)cc2Cl)cc(Oc2ccc(Cl)cc2Cl)c1Cl</smiles>

24

The nucleophilic aromatic substitution was performed according to the general procedure in section 2.1. 2,4-Dichloro-1-(2,4-dichlorophenoxy)-5-iodo-3-methoxybenzene (23) was synthesized in $66 \%$ yield $(276 \mathrm{mg}, 0.59 \mathrm{mmol})$ and 4,4 - $-((4,6$-dichloro-5-methoxy-1,3phenylene)bis(oxy))bis(1,3-dichlorobenzene) (24) in $4 \%$ yield (20.0 $\mathrm{mg}, 0.04 \mathrm{mmol})$. Therefore, the reaction was performed with a total iodononium salt conversion of $70 \%$. Both compounds were obtained as yellowish solids.

\section{2,4-Dichloro-1-(2,4-dichlorophenoxy)-5-iodo-3-methoxybenzene (23)}<smiles>COc1c(Cl)c(I)cc(Oc2ccc(Cl)cc2Cl)c1Cl</smiles>

23

${ }^{1} \mathrm{H}-\mathrm{NMR}\left(300 \mathrm{MHz}, \mathrm{CDCl}_{3}\right): \delta=7.50(\mathrm{~d}, J=2.5 \mathrm{~Hz}, 1 \mathrm{H}), 7.24(\mathrm{dd}, J=8.8,2.5 \mathrm{~Hz}, 1 \mathrm{H}), 7.06(\mathrm{~s}$, $1 \mathrm{H}), 6.88(\mathrm{~d}, J=8.7 \mathrm{~Hz}, 1 \mathrm{H}), 3.93(\mathrm{~s}, 3 \mathrm{H}) .{ }^{13} \mathrm{C}-\mathrm{NMR}\left(75 \mathrm{MHz}, \mathrm{CDCl}_{3}\right): \delta=153.8,152.2,150.1$, 131.0, 130.6, 129.8, 128.5, 126.6, 124.4, 121.4, 121.2, 95.8, 61.0. MS (ESI+): $\mathrm{m} / \mathrm{z}=924.5$ $[2 \mathrm{M}+\mathrm{H}]^{+}$. HRMS (APCl-TOF) $\mathrm{m} / \mathrm{z}$ : [M-H] ${ }^{-}$calcd for $\mathrm{C}_{13} \mathrm{H}_{6} \mathrm{Cl}_{4} \mathrm{IO}_{2} 462.8143$ (100\%), 460.8172 (78\%), 464.8113 (48\%), 463.8176 (14\%); found 462.8125 (100\%), 460.8119 (76\%), 464.8159 (59\%), 463.8155 (14\%). 


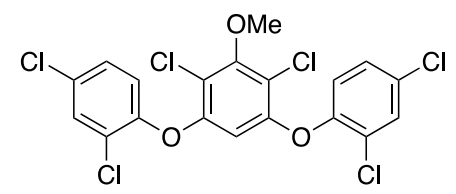

24

${ }^{1} \mathrm{H}-\mathrm{NMR}\left(300 \mathrm{MHz}, \mathrm{CDCl}_{3}\right): \delta=7.22(\mathrm{~d}, J=2.5 \mathrm{~Hz}, 2 \mathrm{H}$ ), 7.17 (dd, $J=8.7,2.5 \mathrm{~Hz}, 2 \mathrm{H}$ ), 6.77 (d, $J=8.8 \mathrm{~Hz}, 2 \mathrm{H}), 6.23(\mathrm{~s}, 1 \mathrm{H}), 3.99(\mathrm{~s}, 3 \mathrm{H}) .{ }^{13} \mathrm{C}-\mathrm{NMR}\left(75 \mathrm{MHz}, \mathrm{CDCl}_{3}\right): \delta=155.4,151.5,150.4$, $130.9,130.1,128.3,125.9,120.0,116.8,106.1,61.2$. MS (ESI+): $\mathrm{m} / \mathrm{z}=992.5[2 \mathrm{M}+\mathrm{H}]^{+}$. HRMS (ESI-TOF) $m / z$ : $[\mathrm{M}+\mathrm{Na}]^{+}$calcd for $\mathrm{C}_{19} \mathrm{H}_{10} \mathrm{Cl}_{6} \mathrm{NaO}_{3} 520.8624$ (100\%), 522.8595 (80\%), 518.8654 (52\%), 524.8565 (34\%); found 520.8622 (100\%), 522.8593 (83\%), 518.8646 (52\%), 524.8564 (36\%).

\section{2,6-Dichloro-3,5-bis(2,4-dichlorophenoxy)phenol - ambigol C (3)}<smiles>COc1c(Cl)c(Oc2ccc(Cl)cc2Cl)c(Cl)c(Oc2ccc(Cl)cc2Cl)c1Cl</smiles><smiles>Oc1c(Cl)c(Oc2ccc(Cl)cc2Cl)cc(Oc2ccc(Cl)cc2Cl)c1Cl</smiles>

Compound $\mathbf{3}$ was synthesized following the general procedure in section 2.1 and isolated as a white solid ( $21.8 \mathrm{mg}, 45.0 \mu \mathrm{mol}, 75 \%)$.

${ }^{1} \mathrm{H}-\mathrm{NMR}\left(300 \mathrm{MHz}, \mathrm{CDCl}_{3}\right): \delta=7.44(\mathrm{~d}, J=2.5 \mathrm{~Hz}, 2 \mathrm{H}), 7.17(\mathrm{dd}, J=8.8,2.5 \mathrm{~Hz}, 2 \mathrm{H}), 6.79$ (d, $J=8.7 \mathrm{~Hz}, 2 \mathrm{H}), 6.17$ (bs, $1 \mathrm{H}), 6.01(\mathrm{~s}, 1 \mathrm{H}) .{ }^{13} \mathrm{C}-\mathrm{NMR}\left(75 \mathrm{MHz}, \mathrm{CDCl}_{3}\right): \delta=151.4,150.7,150.3$, $130.9,130.2,128.3,126.0,120.2,108.4,101.9 .{ }^{1} \mathrm{H}-\mathrm{NMR}\left(300 \mathrm{MHz}, \mathrm{MeOD}-\mathrm{d}_{4}\right): \delta=7.48(\mathrm{~d}$, $J=2.6 \mathrm{~Hz}, 2 \mathrm{H}$ ), $7.24(\mathrm{dd}, J=8.8,2.5 \mathrm{~Hz}, 2 \mathrm{H}), 6.87(\mathrm{~d}, J=8.7 \mathrm{~Hz}, 2 \mathrm{H}), 5.96(\mathrm{~s}, 1 \mathrm{H}) .{ }^{13} \mathrm{C}-\mathrm{NMR}$ $\left(75 \mathrm{MHz}, \mathrm{MeOD}-\mathrm{d}_{4}\right): \delta=153.8,152.7,151.8,131.5,130.9,129.5,126.8,121.5,110.5,102.1$. MS (ESI-): $\mathrm{m} / \mathrm{z}=518.9[\mathrm{M}+\mathrm{K}-2 \mathrm{H}]^{-}, 516.9[\mathrm{M}+\mathrm{Cl}]^{-}, 489.9[\mathrm{M}-\mathrm{H}]^{-}$. HRMS (ESI-TOF) $\mathrm{m} / \mathrm{z}$ : $[\mathrm{M}+\mathrm{Na}]^{+}$calcd for $\mathrm{C}_{18} \mathrm{H}_{8} \mathrm{Cl}_{6} \mathrm{NaO}_{3} 506.8473$ (100\%), 508.8443 (80\%), 504.8502 (52\%), 510.8414 (34\%); found 506.8460 (100\%), 508.8434 (75\%), 504.8478 (47\%), 510.8405 (34\%). HPLC: $\mathrm{t}_{\mathrm{R}}=20.60 \mathrm{~min}$.

\section{3,5-Dihydroxyphenyl acetate (26)}

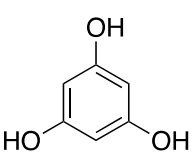

25

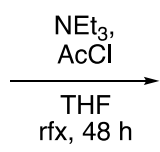

$\mathrm{rfx}, 48 \mathrm{~h}$

Phloroglucinol (25, $3.00 \mathrm{~g}, 23.8 \mathrm{mmol}, 1.0$ eq.) was dissolved in THF ( $42.8 \mathrm{~mL}, 1.8 \mathrm{~mL} / \mathrm{mmol})$ and the solution was cooled to $0{ }^{\circ} \mathrm{C}$. Triethylamine $(6.63 \mathrm{~mL}, 4.82 \mathrm{~g}, 47.6 \mathrm{mmol}, 2.0$ eq.) was added and freshly distilled acetyl chloride $(1.61 \mathrm{~mL}, 1.77 \mathrm{~mL}, 22.6 \mathrm{mmol}, 0.95$ eq.) was added dropwise over $2.5 \mathrm{~h}$. The reaction mixture was stirred for $48 \mathrm{~h}$ under reflux. After treating the solution with $1 \mathrm{~N} \mathrm{HCl}$, the obtained layers were separated. The aqueous layer was extracted with EtOAc (3x). The combined organic layers were washed with brine, dried over $\mathrm{Na}_{2} \mathrm{SO}_{4}$ and filtered. The solvent was removed in vacuo. The crude product was purified by MPLC to give 
the desired product $(\mathbf{2 6}, 687 \mathrm{mg}, 4.08 \mathrm{mmol}, \mathbf{1 8 \%})$ as a colorless oil. The substrate $(\mathbf{2 5}, 1.17 \mathrm{~g}$, $9.28 \mathrm{mmol}$ ) was re-isolated in 39\% yield, based on which 26 was obtained in $30 \%$ isolated yield. The diacetylated product $(1.22,5.80 \mathrm{mmol}, 26 \%$, brsm: $40 \%)$ was also isolated during MPLC purification. Analytical data were in agreement with literature values. ${ }^{11}$

${ }^{1} \mathrm{H}-\mathrm{NMR}\left(300 \mathrm{MHz}, \mathrm{CDCl}_{3}\right): \delta=9.48$ (bs, $\left.2 \mathrm{H}\right), 6.09$ (t, $\left.J=2.1 \mathrm{~Hz}, 1 \mathrm{H}\right), 5.94(\mathrm{~d}, J=2.1 \mathrm{~Hz}, 2 \mathrm{H}$ ), $2.19(\mathrm{~s}, 3 \mathrm{H}) . \mathbf{M S}(\mathrm{ESI}+): \mathrm{m} / \mathrm{z}=233.0[\mathrm{M}+\mathrm{Na}]^{+}, 169.1[\mathrm{M}+\mathrm{Na}]^{+} . \mathbf{M S}(\mathrm{ESI}-): \mathrm{m} / \mathrm{z}=166.9[\mathrm{M}-\mathrm{H}]^{-}$.

\section{2,6-Dichloro-3,5-dihydroxyphenyl acetate (27)}
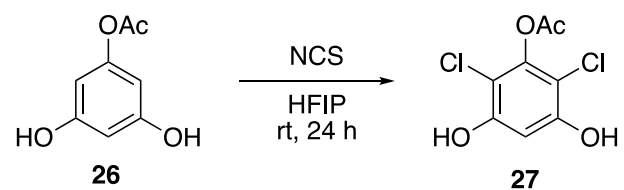

The chlorination was prepared according to a published procedure. ${ }^{12}$ Compound 26 (250 mg, $1.37 \mathrm{mmol}, 1.0$ eq.) was dissolved in HFIP $(1.4 \mathrm{~mL}, 1.0 \mathrm{~mL} / \mathrm{mmol})$. After addition of NCS ( $457 \mathrm{mg}, 3.43 \mathrm{mmol}, 2.5 \mathrm{eq}$.), the reaction was stirred for $24 \mathrm{~h}$ at room temperature. The reaction solution was evaporated and the obtained residue was purified by MPLC to give the desired product $(\mathbf{2 7}, 96.0 \mathrm{mg}, 0.41 \mathrm{mmol}, 30 \%)$ as a yellowish oil. The substrate $(26,92 \mathrm{mg}$, $0.55 \mathrm{mmol}$ ) was re-isolated in $40 \%$ yield, based on which the desired product $\mathbf{2 7}$ was obtained in $70 \%$ isolated yield. The trichlorinated product ( $56 \mathrm{mg}, 0.21 \mathrm{mmol}, 15 \%$, brsm: $26 \%$ ) was also isolated during MPLC purification.

${ }^{1} \mathrm{H}-\mathrm{NMR}\left(300 \mathrm{MHz}, \mathrm{CDCl}_{3}\right): \delta=6.62(\mathrm{~s}, 1 \mathrm{H}), 4.79$ (bs, $\left.2 \mathrm{H}\right), 2.41(\mathrm{~s}, 3 \mathrm{H}) .{ }^{13} \mathrm{C}-\mathrm{NMR}(75 \mathrm{MHz}$, $\left.\mathrm{CDCl}_{3}\right): \delta=167.4,151.6,144.1,107.1,101.5,20.4 . \mathrm{MS}(\mathrm{ESI}+): \mathrm{m} / \mathrm{z}=259.0[\mathrm{M}+\mathrm{Na}]^{+}, 236.6$ $[\mathrm{M}+\mathrm{H}]^{+}$. HRMS (ESI-TOF) $\mathrm{m} / \mathrm{z}$ : $[\mathrm{M}+\mathrm{Na}]^{+}$calcd for $\mathrm{C}_{8} \mathrm{H}_{6} \mathrm{Cl}_{2} \mathrm{NaO}_{4} 258.9536$ (100\%), 260.9506 (64\%), 262.9477 (10\%); found 258.9529 (100\%), 260.9502 (71\%), 262.9477 (12\%). TLC: $R_{f}(n-$ pentane $/$ EtOAc $=2: 1)=0.45$.

\section{(2,4-Dichlorophenyl)(2,4,6-trimethoxyphenyl)iodonium 4-methylbenzenesulfonate (28)}
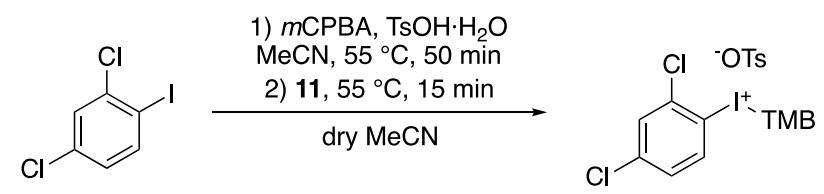

28

Compound $\mathbf{2 8}$ was synthesized following the general procedure in section 2.1 and isolated as a white solid (4.10 g, $6.61 \mathrm{mmol}, 92 \%)$.

${ }^{1} \mathrm{H}-\mathrm{NMR}\left(300 \mathrm{MHz}, \mathrm{DMSO}-\mathrm{d}_{6}\right): \delta=8.18(\mathrm{~d}, J=8.6 \mathrm{~Hz}, 1 \mathrm{H}), 7.96(\mathrm{~d}, J=2.3 \mathrm{~Hz}, 1 \mathrm{H}$ ), 7.52-7.44 $(\mathrm{m}, 3 \mathrm{H}), 7.10(\mathrm{~d}, J=8.2 \mathrm{~Hz}, 1 \mathrm{H}), 6.44(\mathrm{~s}, 2 \mathrm{H}), 3.93(\mathrm{~s}, 6 \mathrm{H}), 3.85(\mathrm{~s}, 3 \mathrm{H}), 2.28(\mathrm{~s}, 3 \mathrm{H}) .{ }^{13} \mathrm{C}-$ NMR $\left(75 \mathrm{MHz}\right.$, DMSO-d $\left.\mathrm{d}_{6}\right): \delta=166.3,159.4,145.7,139.9,138.1,137.6,137.2,129.8,129.7$, 128.1, 125.5, 117.5, 92.1, 87.5, 57.2, 56.2, 20.8. MS (ESI+): $\mathrm{m} / \mathrm{z}=649.2[\mathrm{M}+\mathrm{K}]^{+}, 611.0[\mathrm{M}+\mathrm{H}]^{+}$, 
439.0 [M-OTs] $]^{+}$MS (ESI-): $\mathrm{m} / \mathrm{z}=609.0[\mathrm{M}-\mathrm{H}]^{-}, 630.7[\mathrm{M}+\mathrm{Na}-2 \mathrm{H}]^{-}$. HRMS (ESI-TOF) $\mathrm{m} / \mathrm{z}$ : [MOTs $]^{+}$calcd for $\mathrm{C}_{15} \mathrm{H}_{14} \mathrm{Cl}_{2} \mathrm{IO}_{3} 438.9360$ (100\%), 440.9330 (64\%), 439.9393 (16\%); found 438.9345 (100\%), 440.9318 (62\%), 439.9399 (14\%).

\section{2,6-Dichloro-3,5-bis(2,4-dichlorophenoxy)phenol - ambigol C (3)}

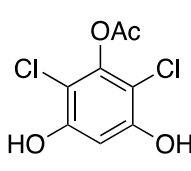

27

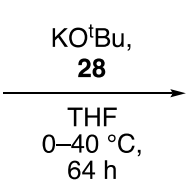

$64 \mathrm{~h}$<smiles>Oc1c(Oc2ccc(Cl)cc2Cl)cc(Oc2ccc(Cl)cc2Cl)c(Cl)c1Cl</smiles>

3

Ambigol C (3) was synthesized following the general procedure in section 2.1 and isolated as a white solid $(23.0 \mathrm{mg}, 47.4 \mu \mathrm{mol}, 14 \%)$. Analytical data were in agreement to the other synthesis route.

\section{2,3',4,5'-Tetrachloro-5-(2,4-dichlorophenoxy)-2',3-dimethoxy-1,1'-biphenyl (29)}<smiles>COc1c(Cl)c(I)cc(Oc2ccc(Cl)cc2Cl)c1Cl</smiles>

23

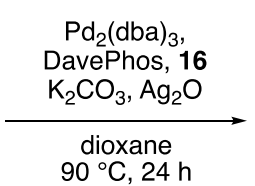

$90^{\circ} \mathrm{C}, 24 \mathrm{~h}$<smiles>COc1c(Cl)cc(Cl)cc1-c1cc(Oc2ccc(Cl)cc2Cl)c(OC)c(Cl)c1Cl</smiles>

29

The Suzuki coupling was performed according to the general procedure in section 2.1. The product 29 was obtained in $62 \%$ yield $(239 \mathrm{mg}, 0.47 \mathrm{mmol}$ ) as a yellowish solid.

${ }^{1} \mathrm{H}-\mathrm{NMR}\left(300 \mathrm{MHz}, \mathrm{CDCl}_{3}\right): \delta=7.47(\mathrm{~d}, J=2.5 \mathrm{~Hz}, 1 \mathrm{H}), 7.41(\mathrm{~d}, J=2.5 \mathrm{~Hz}, 1 \mathrm{H}), 7.22(\mathrm{dd}, J=8.7$, $2.5 \mathrm{~Hz}, 1 \mathrm{H}$ ), $7.07(\mathrm{~d}, J=2.5 \mathrm{~Hz}, 1 \mathrm{H}), 6.92(\mathrm{~d}, J=8.7 \mathrm{~Hz}, 1 \mathrm{H}), 6.53(\mathrm{~s}, 1 \mathrm{H}) 4.00(\mathrm{~s}, 3 \mathrm{H}), 3.53$ (s, $3 \mathrm{H}) .{ }^{13} \mathrm{C}-\mathrm{NMR}\left(75 \mathrm{MHz}, \mathrm{CDCl}_{3}\right): \delta=154.3,152.5,151.6,150.2,135.3,134.4,131.0,130.57$, $130.55,129.5,129.3,129.2,128.5,126.6,123.8,121.3,120.7,115.6,61.2,61.1$. MS (ESI+): $\mathrm{m} / \mathrm{z}=1043.0[2 \mathrm{M}+\mathrm{Na}]^{+}, 2021.1[2 \mathrm{M}+\mathrm{H}]^{+}, 548.4[\mathrm{M}+\mathrm{K}]^{+}, 532.5[\mathrm{M}+\mathrm{Na}]^{+}$. HRMS (ESI-TOF) $\mathrm{m} / \mathrm{z}:$ $[\mathrm{M}+\mathrm{Na}]^{+}$calcd for $\mathrm{C}_{20} \mathrm{H}_{12} \mathrm{Cl}_{6} \mathrm{NaO}_{3} 534.8781$ (100\%), 536.8751 (80\%), 532.8810 (52\%), 538.8722 (34\%); found 534.8770 (100\%), 536.8750 (80\%), 432.8810 (54\%), 538.8725 (34\%).

2',3,4',5-Tetrachloro-5'-(2,4-dichlorophenoxy)-[1,1'-biphenyl]-2,3'-diol (30)<smiles>COc1c(Cl)cc(Cl)cc1-c1cc(Oc2ccc(Cl)cc2Cl)c(OC)c(Cl)c1Cl</smiles>

29

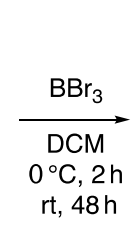

$\mathrm{rt}, 48 \mathrm{~h}$<smiles>Oc1c(Cl)cc(Cl)cc1-c1cc(Oc2ccc(Cl)cc2Cl)c(O)c(Cl)c1Cl</smiles>

30

Compound $\mathbf{3 0}$ was synthesized following the general procedure in section 2.1 and isolated as a white solid (148 mg, $305 \mu \mathrm{mol}, 69 \%$ ).

${ }^{1} \mathrm{H}-\mathrm{NMR}\left(300 \mathrm{MHz}, \mathrm{CDCl}_{3}\right): \delta=7.47(\mathrm{~d}, J=2.3 \mathrm{~Hz}, 1 \mathrm{H}), 7.37(\mathrm{~d}, J=2.5 \mathrm{~Hz}, 1 \mathrm{H}), 7.21(\mathrm{dd}, J=8.6$, $2.3 \mathrm{~Hz}, 1 \mathrm{H}$ ), 7.04 (d, J = 2.5 Hz, 1 H), 6.95 (d, J = 8.8 Hz, 1 H), $6.36(\mathrm{~s}, 1 \mathrm{H}), 6.21$ (bs, $1 \mathrm{H}), 5.64$ 
(bs, $1 \mathrm{H}$ ). ${ }^{13} \mathrm{C}-\mathrm{NMR}\left(75 \mathrm{MHz}, \mathrm{CDCl}_{3}\right): \delta=151.5,150.1,149.7,147.6,134.3,130.9,130.4,129.5$, 129.0, 128.4, 126.9, 126.6, 125.3, 121.4, 121.2, 115.9, 112.4, 111.7. MS (ESI-): $\mathrm{m} / \mathrm{z}=480.7$ $[\mathrm{M}-\mathrm{H}]^{-}$. HRMS (ESI-TOF) $\mathrm{m} / \mathrm{z}$ : [M-H] $]^{-}$calcd for $\mathrm{C}_{18} \mathrm{H}_{7} \mathrm{Cl}_{6} \mathrm{O}_{3} 482.8502$ (100\%), 484.8472 (80\%), 480.8531 (52\%), 486.8443 (34\%), 483.8535 (20\%); found 482.8492 (100\%), 484.8465 (80\%), $480.8523(52 \%), 486.8440$ (33\%), $483.8524(17 \%)$. HPLC: $t_{R}=17.56 \mathrm{~min}$.

\subsection{Synthesis of Ambigol D (4)}

\section{1,3-Dichloro-2,4-diiodo-5-methoxybenzene (32)}

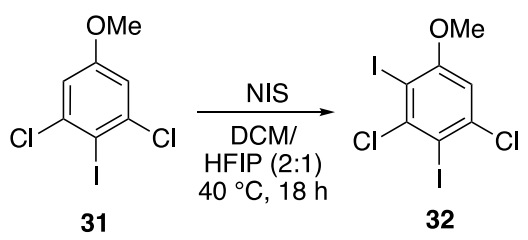

The iodination was conducted according to a published procedure. ${ }^{12}$ 3,5-Dichloro-4iodoanisole $(31,1.00 \mathrm{~g}, 3.30 \mathrm{mmol}, 1.0$ eq.) was dissolved in a mixture of DCM/HFIP $(15.0 \mathrm{~mL}$, 2:1) and NIS ( $0.75 \mathrm{~g}, 3.30 \mathrm{mmol}, 1.0 \mathrm{eq}$.) was added. The reaction mixture was stirred for $24 \mathrm{~h}$ at room temperature before being filtered and dried. The obtained residue was purified by column chromatography ( $\mathrm{n}$-pentane/EtOAc $=15: 1$ ) giving $32(665 \mathrm{mg}, 1.55 \mathrm{mmol}$ ) in 47\% yield as yellowish oil.

${ }^{1} \mathrm{H}-\mathrm{NMR}\left(300 \mathrm{MHz}, \mathrm{CDCl}_{3}\right): \delta=6.85(\mathrm{~s}, 1 \mathrm{H}), 3.89(\mathrm{~s}, 3 \mathrm{H}) .{ }^{13} \mathrm{C}-\mathrm{NMR}\left(75 \mathrm{MHz}, \mathrm{CDCl}_{3}\right): \delta=160.0$, 144.54, 141.0, 109.4, 91.7, 88.6, 57.3. MS $(E S I+): m / z=452.3[\mathrm{M}+\mathrm{Na}]^{+}, 430.5[\mathrm{M}+\mathrm{H}]^{+}$. MS (ESI-): $\mathrm{m} / \mathrm{z}=467.0[\mathrm{M}+\mathrm{K}-2 \mathrm{H}]^{-}, 428.8[\mathrm{M}-\mathrm{H}]^{-}$. HRMS (APCl-TOF) $\mathrm{m} / \mathrm{z}:[\mathrm{M}]^{+}$calcd for $\mathrm{C}_{7} \mathrm{H}_{4} \mathrm{Cl}_{2} \mathrm{I}_{2} \mathrm{O} 427.7729$ (100\%), 429.7699 (64\%), 428.7762 (8\%); found 427.7717 (100\%), 429.7687 (83\%), 428.7786 (7\%). TLC: $R_{f}(n-p e n t a n e / E t O A C=10: 1)=0.69$.

\section{(2,4-Dichloro-6-methoxy-1,3-phenylene)bis((2,4,6-trimethoxyphenyl)iodonium) Bistosylat} (33)
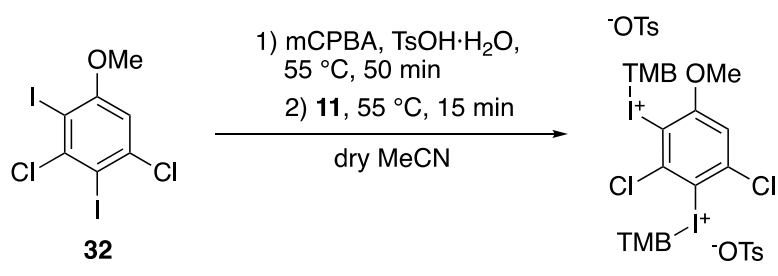

33

The diiodonium salt $\mathbf{3 3}$ was prepared according to the general procedure in section 2.1. The salt was synthesized in $43 \%$ ( $560 \mathrm{mg}, 0.51 \mathrm{mmol}$ ) yield as a beige solid.

${ }^{1} \mathrm{H}-\mathrm{NMR}\left(300 \mathrm{MHz}, \mathrm{DMSO}-\mathrm{d}_{6}\right): \delta=8.47(\mathrm{~d}, J=7.2 \mathrm{~Hz}, 4 \mathrm{H}), 7.11(\mathrm{~d}, J=7.5 \mathrm{~Hz}, 4 \mathrm{H}$ ), 6.47-6.37 $(\mathrm{m}, 3 \mathrm{H}), 5.96(\mathrm{~s}, 2 \mathrm{H}), 3.91(\mathrm{~s}, 3 \mathrm{H}), 3.89(\mathrm{~s}, 9 \mathrm{H}), 3.84(\mathrm{~s}, 3 \mathrm{H}), 3.74(\mathrm{~s}, 6 \mathrm{H}), 2.28(\mathrm{~s}, 6 \mathrm{H}) . \mathrm{MS}$ $(\mathrm{ESI}+): \mathrm{m} / \mathrm{z}=1149.1[\mathrm{M}+2 \mathrm{Na}-\mathrm{H}]^{+}, 1127.3[\mathrm{M}+\mathrm{Na}]^{+}$. HRMS (ESI-TOF) $\mathrm{m} / \mathrm{z}:[\mathrm{M}+\mathrm{Na}]^{+}$cald for 
$\mathrm{C}_{39} \mathrm{H}_{40} \mathrm{Cl}_{2} \mathrm{I}_{2} \mathrm{NaO}_{13} \mathrm{~S}_{2} 1126.9269$ (100\%), 1128.9239 (64\%), 1127.9303 (42\%), 1129.9273 (27\%), 1130.9210 (10\%); found 1126.9276 (100\%), 1128.9254 (87\%), 1127.9308 (53\%), 1129.9288 (37\%), 1130.9248 (29\%).

\section{1,3-Dichloro-4-(2,4-dichlorophenoxy)-2-iodo-5-methoxybenzene (34)}

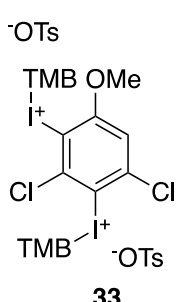

33<smiles>COc1cc(Cl)c(I)c(Oc2ccc(Cl)cc2Cl)c1Cl</smiles>

34

Compound $\mathbf{3 4}$ was synthesized following the general procedure in section 2.1 and isolated as a white solid (85.0 $\mathrm{mg}, 0.18 \mathrm{mmol}, 61 \%)$.

${ }^{1} \mathrm{H}-\mathrm{NMR}\left(300 \mathrm{MHz}, \mathrm{CDCl}_{3}\right): \delta=7.44(\mathrm{~d}, J=2.5 \mathrm{~Hz}, 1 \mathrm{H}), 7.12(\mathrm{~s}, 1 \mathrm{H}), 7.05$ (dd, J= 8.8, $2.5 \mathrm{~Hz}$, $1 \mathrm{H}), 6.39(\mathrm{~d}, J=8.8 \mathrm{~Hz}, 1 \mathrm{H}), 3.78(\mathrm{~s}, 3 \mathrm{H}) .{ }^{13} \mathrm{C}-\mathrm{NMR}\left(75 \mathrm{MHz}, \mathrm{CDCl}_{3}\right): \delta=152.4,151.5,138.3$, $137.2,135.1,130.5,127.8,127.6,123.5,115.1,112.3,93.25,56.8 . \mathrm{MS}(\mathrm{ESI}+): \mathrm{m} / \mathrm{z}=946.4$ $[2 \mathrm{M}+\mathrm{Na}]^{+}, 500.8[\mathrm{M}+\mathrm{K}]^{+}, 484.9[\mathrm{M}+\mathrm{Na}]^{+} . \mathrm{MS}(\mathrm{ESI}-): \mathrm{m} / \mathrm{z}=497.1[\mathrm{M}+\mathrm{Cl}]^{-}, 460.4[\mathrm{M}-\mathrm{H}]^{-}$. HRMS (ESI-TOF) $m / z$ : $[\mathrm{M}+\mathrm{Na}]^{+}$calcd for $\mathrm{C}_{13} \mathrm{H}_{7} \mathrm{Cl}_{4} \mathrm{INaO}_{2} 486.8113$ (100\%), 484.8143 (78\%), 488.8084 (48\%), 487.8147 (14\%), 485.8176 (11\%); found 486.8103 (100\%), 484.8135 (80\%), 488.8078 (47\%), 487.8134 (14\%), 485.8169 (10\%).

\section{2,3',5',6-Tetrachloro-3-(2,4-dichlorophenoxy)-2',4-dimethoxy-1,1'-biphenyl (35)}
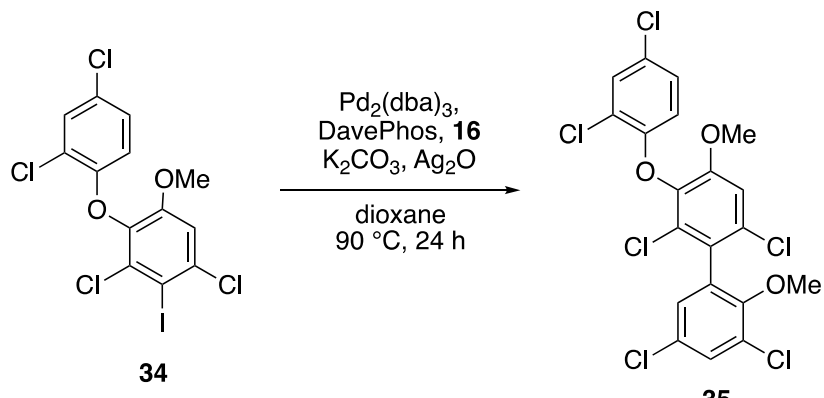

The Suzuki coupling was performed according to the general procedure in section 2.1. The product 35 was obtained in $54 \%$ yield $(48.0 \mathrm{mg}, 93.6 \mu \mathrm{mol})$ as a white solid. The substrate (34, $23.9 \mathrm{mg}, 51.6 \mu \mathrm{mol}$ ) was re-isolated in $30 \%$ yield, based on which the desired product was obtained in $74 \%$ isolated yield.

${ }^{1} \mathrm{H}-\mathrm{NMR}\left(300 \mathrm{MHz}, \mathrm{CDCl}_{3}\right): \delta=7.47(\mathrm{~d}, J=2.5 \mathrm{~Hz}, 1 \mathrm{H}), 7.45(\mathrm{~d}, J=2.5 \mathrm{~Hz}, 1 \mathrm{H}), 7.12-7.05(\mathrm{~m}$, $3 \mathrm{H}), 6.46(\mathrm{~d}, J=7.2,8.8 \mathrm{~Hz}, 1 \mathrm{H}), 3.84(\mathrm{~s}, 3 \mathrm{H}), 3.63(\mathrm{~s}, 3 \mathrm{H}) .{ }^{13} \mathrm{C}-\mathrm{NMR}\left(75 \mathrm{MHz}, \mathrm{CDCl}_{3}\right): \delta=$ 153.2, 152.9, 151.7, 138.9, 133.0, 131.9, 130.9, 130.5, 129.98, 129.95, 129.4, 129.2, 127.9, 127.7, 127.6, 123.6, 115.0, 112.5, 61.1, 56.7. MS (ESI+): $\mathrm{m} / \mathrm{z}=1020.9[2 \mathrm{M}+\mathrm{H}]^{+}, 548.6[\mathrm{M}+\mathrm{K}]^{+}$, $533.0[\mathrm{M}+\mathrm{Na}]^{+}, 510.9[\mathrm{M}+\mathrm{H}]^{+} . \mathrm{MS}(\mathrm{ESI}-): \mathrm{m} / \mathrm{z}=1018.5[2 \mathrm{M}-\mathrm{H}]^{-}, 544.8[\mathrm{M}+\mathrm{Cl}]^{-}, 508.4[\mathrm{M}-\mathrm{H}]^{-}$. 
HRMS (ESI-TOF) $m / z$ : $[\mathrm{M}+\mathrm{Na}]^{+}$calcd for $\mathrm{C}_{20} \mathrm{H}_{12} \mathrm{Cl}_{6} \mathrm{NaO}_{3} 534.8786$ (100\%), 536.8756 (80\%), 532.8815 (52\%), 538.8727 (34\%); found 534.8782 (100\%), 536.8750 (82\%), 532.8843 (55\%), $538.8723(35 \%)$.

\section{2',3,5,6'-Tetrachloro-3'-(2,4-dichlorophenoxy)-[1,1'-biphenyl]-2,4'-diol - Ambigol D (4)}

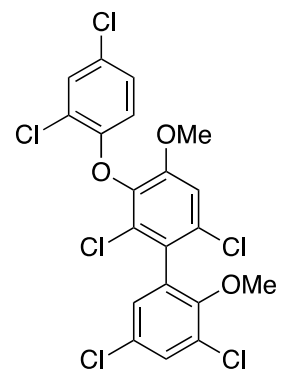

35
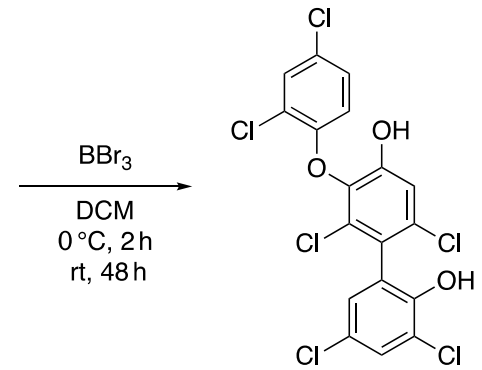

Compound 4 was synthesized following the general procedure in section 2.1 and isolated as a white solid (39.2 mg, $80.8 \mu \mathrm{mol}, 92 \%)$. Analytical data were in agreement with literature values. ${ }^{13}$

${ }^{1} \mathrm{H}-\mathrm{NMR}\left(300 \mathrm{MHz}, \mathrm{CDCl}_{3}\right): \delta=7.44(\mathrm{~d}, J=2.5 \mathrm{~Hz}, 1 \mathrm{H}), 7.41(\mathrm{~d}, J=2.5 \mathrm{~Hz}, 1 \mathrm{H}), 7.20(\mathrm{~s}, 1 \mathrm{H})$, $7.13(\mathrm{dd}, J=8.8,2.5 \mathrm{~Hz}, 1 \mathrm{H}), 7.05(\mathrm{~d}, J=2.5 \mathrm{~Hz}, 1 \mathrm{H}), 6.60(\mathrm{~d}, J=8.8 \mathrm{~Hz}, 1 \mathrm{H}), 5.89$ (bs, $1 \mathrm{H})$, 5.57 (bs, $1 \mathrm{H}$ ). ${ }^{13} \mathrm{C}-\mathrm{NMR}\left(75 \mathrm{MHz}, \mathrm{CDCl}_{3}\right): \delta=150.9,150.0,148.1,137.4,132.5,130.8,130.3$, 129.2, 129.1, 128.9, 128.1, 127.4, 125.38, 125.35, 123.8, 121.1, 116.6, 115.7. ${ }^{1} \mathrm{H}-\mathrm{NMR}$ $\left(300 \mathrm{MHz}, \mathrm{MeOD}-\mathrm{d}_{4}\right): \delta=7.49(\mathrm{~d}, J=2.5 \mathrm{~Hz}, 1 \mathrm{H}), 7.41(\mathrm{~d}, J=2.6 \mathrm{~Hz}, 1 \mathrm{H}), 7.17$ (dd, $J=8.9$, $2.5 \mathrm{~Hz}, 1 \mathrm{H}), 7.10(\mathrm{~s}, 1 \mathrm{H}), 7.02(\mathrm{~d}, J=2.6 \mathrm{~Hz}, 1 \mathrm{H}), 6.66(\mathrm{~d}, J=8.9 \mathrm{~Hz}, 1 \mathrm{H}) .{ }^{13} \mathrm{C}-\mathrm{NMR}(75 \mathrm{MHz}$, MeOD-d $\left.\mathrm{d}_{4}\right): \delta=153.2,152.8,151.2,139.0,132.9,131.0,130.90,130.85,130.3,128.7,128.5$, $128.3,127.9,125.2,124.4,123.4,117.4,116.7 . M S(E S I+): m / z=1002.7[2 M+K], 964.9$ $[2 \mathrm{M}+\mathrm{H}]^{+}, 526.7[\mathrm{M}+2 \mathrm{Na}-\mathrm{H}]^{+}, 520.9[\mathrm{M}+\mathrm{K}]^{+}, 504.9[\mathrm{M}+\mathrm{Na}]^{+}, 483.1[\mathrm{M}+\mathrm{H}]^{+}$. HRMS (ESI-TOF) $\mathrm{m} / \mathrm{z}:[\mathrm{M}+\mathrm{Na}]^{+}$calcd for $\mathrm{C}_{18} \mathrm{H}_{8} \mathrm{Cl}_{6} \mathrm{NaO}_{3} 506.8473$ (100\%), 508.8443 (80\%), 504.8502 (52\%), 510.8414 (34\%); found 506.8462 (100\%), 508.8432 (80\%), $504.8486(56 \%), 510.8407$ (33\%). HPLC: $t_{R}=19.39 \mathrm{~min}$.

\subsection{Synthesis of Ambigol E (5)}

\section{2-Chloro-6-iodo-3-methoxyphenol (37)}

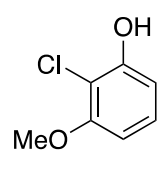

36

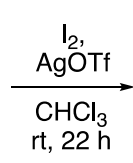

$\mathrm{rt}, 22 \mathrm{~h}$

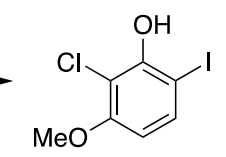

37

The iodination was prepared conducted to a published procedure. ${ }^{14}$ 2-Chloro-3-methoxyphenol (36, $264 \mathrm{mg}, 1.66 \mathrm{mmol}, 1.0$ eq.) and silver trifluoromethanesulfonate $(368 \mathrm{mg}$, $1.66 \mathrm{mmol}, 1.0$ eq.) were dissolved with $\mathrm{CHCl}_{3}(8.3 \mathrm{~mL}, 5.0 \mathrm{~mL} / \mathrm{mmol})$. A solution of iodine (423 mg, $1.66 \mathrm{mmol}, 1.0$ eq.) in $\mathrm{CHCl}_{3}(1.7 \mathrm{~mL}, 1.0 \mathrm{~mL} / \mathrm{mmol}$ ) was added dropwise to the 
reaction suspension over one hour. The reaction mixture was stirred for $22 \mathrm{~h}$ at room temperature before being treated with sat. $\mathrm{Na}_{2} \mathrm{~S}_{2} \mathrm{O}_{3}$ solution. The obtained layers were separated and the aqueous phase was extracted with DCM (2x). The combined organic layers were washed with brine, dried over $\mathrm{Na}_{2} \mathrm{SO}_{4}$ and filtered. The solvent was removed in vacuo. The crude material was purified by MPLC to give the desired product $(37,334 \mathrm{mg}, 1.17 \mathrm{mmol}$, $70 \%)$ as an orange solid.

${ }^{1} \mathrm{H}-\mathrm{NMR}\left(300 \mathrm{MHz}, \mathrm{CDCl}_{3}\right): \delta=7.54(\mathrm{~d}, J=8.8 \mathrm{~Hz}, 1 \mathrm{H}), 6.37(\mathrm{~d}, J=8.8 \mathrm{~Hz}, 1 \mathrm{H}), 6.01$ (bs, $1 \mathrm{H}$ ), $3.88(\mathrm{~s}, 3 \mathrm{H}) .{ }^{13} \mathrm{C}-\mathrm{NMR}\left(75 \mathrm{MHz}, \mathrm{CDCl}_{3}\right): \delta=156.3,151.7,136.5,108.4,106.1,72.4,56.6$. MS (ESI-): $\mathrm{m} / \mathrm{z}=304.8[\mathrm{M}+\mathrm{Na}-2 \mathrm{H}]^{-}, 282.8[\mathrm{M}-\mathrm{H}]^{-}$. HRMS (ESI-TOF) $\mathrm{m} / \mathrm{z}:[\mathrm{M}+\mathrm{Na}]^{+}$calcd for $\mathrm{C}_{7} \mathrm{H}_{6} \mathrm{ClINaO}_{2} 306.8994$ (100\%), 308.8964 (32\%), 307.9027 (8\%), 309.8998 (2\%); found 306.8988 (100\%), 308.8959 (36\%), 307.9025 (8\%), 309.8996 (4\%).

\section{2-Chloro-3-(2,4-dichlorophenoxy)-4-iodo-1-methoxybenzene (38)}
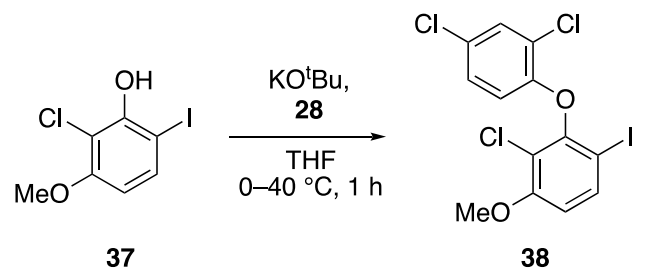

38

Compound 38 was synthesized following the general procedure in section 2.1 and isolated as a white solid (122 $\mathrm{mg}, 0.28 \mathrm{mmol}, 54 \%$ ).

${ }^{1} \mathrm{H}-\mathrm{NMR}\left(300 \mathrm{MHz}, \mathrm{CDCl}_{3}\right): \delta=7.71(\mathrm{~d}, J=8.9 \mathrm{~Hz}, 1 \mathrm{H}), 7.48(\mathrm{~d}, J=2.5 \mathrm{~Hz}, 1 \mathrm{H}), 7.05(\mathrm{dd}, J=8.8$, $2.5 \mathrm{~Hz}, 1 \mathrm{H}), 6.69$ (d, J = 8.9 Hz, $1 \mathrm{H}), 6.33(\mathrm{~d}, J=8.8 \mathrm{~Hz}, 1 \mathrm{H}), 3.93(\mathrm{~s}, 3 \mathrm{H}) .{ }^{13} \mathrm{C}-\mathrm{NMR}(75 \mathrm{MHz}$, $\left.\mathrm{CDCl}_{3}\right): \delta=157.2,151.0,150.7,137.2,130.6,127.7,127.6,123.7,117.6,115.2,111.2,80.3$, 56.8. MS (ESI+): $\mathrm{m} / \mathrm{z}=450.4[\mathrm{M}+\mathrm{Na}]^{+}$. HRMS (ESI-TOF) $\mathrm{m} / \mathrm{z}:[\mathrm{M}+\mathrm{Na}]^{+}$calcd for $\mathrm{C}_{13} \mathrm{H}_{8} \mathrm{Cl}_{3} / \mathrm{NaO}_{2}$ 450.8527 (100\%), 452.8498 (96\%), 454.8468 (30\%); found 450.8518 (100\%), 452.8492 (98\%), 454.8462 (30\%).

\section{3,3',5'-Trichloro-2-(2,4-dichl orophenoxy)-2',4-dimethoxy-1,1'-biphenyl (39)}

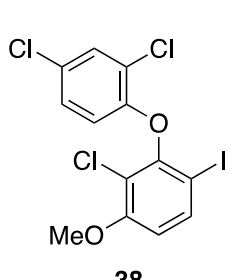

38

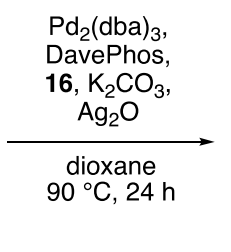

$\sqrt{20}$

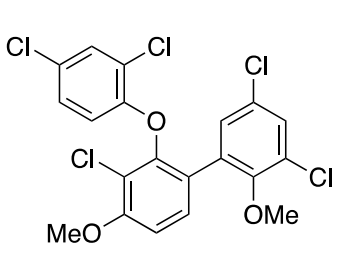

39

The Suzuki coupling was performed according to the general procedure in section 2.1. The product 39 was obtained in $54 \%$ yield $(69.0 \mathrm{mg}, 0.14 \mathrm{mmol}$ ) as a yellowish solid. The substrate 38 ( $23.0 \mathrm{mg}, 0.05 \mathrm{mmol})$ was re-isolated in $20 \%$ yield, based on which the desired product 39 was obtained in $74 \%$ isolated yield. 
${ }^{1} \mathrm{H}-\mathrm{NMR}\left(300 \mathrm{MHz}, \mathrm{CDCl}_{3}\right): \delta=7.34(\mathrm{~d}, J=8.7 \mathrm{~Hz}, 1 \mathrm{H}), 7.30(\mathrm{~d}, J=2.5 \mathrm{~Hz}, 1 \mathrm{H}), 7.29(\mathrm{~d}, J=$ $2.6 \mathrm{~Hz}, 1 \mathrm{H}), 7.12(\mathrm{~d}, J=2.5 \mathrm{~Hz}, 1 \mathrm{H}), 7.00-6.93(\mathrm{~m}, 2 \mathrm{H}), 6.39(\mathrm{~d}, J=8.8 \mathrm{~Hz}, 1 \mathrm{H}), 4.00(\mathrm{~s}, 3 \mathrm{H})$, 3.55 (s, $3 \mathrm{H}$ ). ${ }^{13} \mathrm{C}-\mathrm{NMR}\left(75 \mathrm{MHz}, \mathrm{CDCl}_{3}\right): \delta=156.6,152.9,151.5,149.0,132.3,130.4,130.2$, $130.00,129.98,128.93,128.88,127.5,127.3,123.7,123.4,117.5,115.7,109.0,61.2,56.7 . \mathrm{MS}$ $(\mathrm{ESI}+): \mathrm{m} / \mathrm{z}=476.6[\mathrm{M}+\mathrm{H}]^{+}$. HRMS (ESI-TOF) $\mathrm{m} / \mathrm{z}:[\mathrm{M}+\mathrm{Na}]^{+}$calcd for $\mathrm{C}_{20} \mathrm{H}_{13} \mathrm{Cl}_{5} \mathrm{NaO}_{3} 500.9171$ (100\%), 502.9141 (64\%), 498.9200 (63\%), 501.9204 (22\%); found 500.9164 (100\%), 502.9135 (60\%), 498.9195 (59\%), 501.9195 (21\%).

\section{3,3',5-Trichloro-2'-(2,4-dichlorophenoxy)-[1,1'-biphenyl]-2,4'-diol (40)}

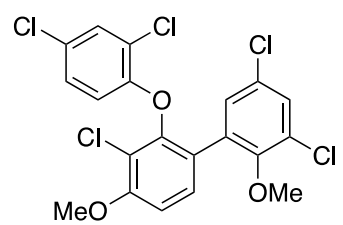

39

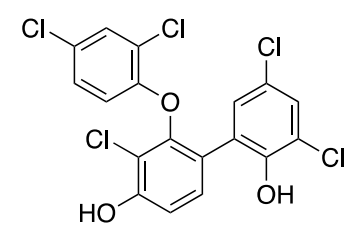

40

Compound $\mathbf{4 0}$ was synthesized following the general procedure in section 2.1 and isolated as a white solid (41.0 mg, $91.0 \mu \mathrm{mol}, 62 \%)$.

${ }^{1} \mathrm{H}-\mathrm{NMR}\left(300 \mathrm{MHz}, \mathrm{CDCl}_{3}\right): \delta=7.31-7.29(\mathrm{~m}, 1 \mathrm{H}), 7.27(\mathrm{~d}, J=1.9 \mathrm{~Hz}, 1 \mathrm{H}), 7.11(\mathrm{~d}, J=2.5 \mathrm{~Hz}$, $1 \mathrm{H}$ ), 7.08 (d, J=8.6 Hz, $1 \mathrm{H}$ ), $6.99(\mathrm{~d}, J=8.8,2.5 \mathrm{~Hz}, 1 \mathrm{H}), 6.43(\mathrm{~d}, J=8.9 \mathrm{~Hz}, 1 \mathrm{H}$ ), 5.61 (bs, $2 \mathrm{H}) .{ }^{13} \mathrm{C}-\mathrm{NMR}\left(75 \mathrm{MHz}, \mathrm{CDCl}_{3}\right): \delta=153.2,151.4,148.4,147.8,130.6,130.2,130.1,128.5$, $127.7,127.4,125.3,125.2,123.4,123.0,121.2,115.8,114.8,113.5 . \mathrm{MS}(\mathrm{ESI}+): \mathrm{m} / \mathrm{z}=918.6$ $[2 \mathrm{M}+\mathrm{Na}]^{+}, 493.0[\mathrm{M}+2 \mathrm{Na}-\mathrm{H}]^{+}, 487.1[\mathrm{M}+\mathrm{K}]^{+}$. HRMS (ESI-TOF) $\mathrm{m} / \mathrm{z}:[\mathrm{M}-\mathrm{H}]^{-}$calcd for $\mathrm{C}_{18} \mathrm{H}_{8} \mathrm{Cl}_{5} \mathrm{O}_{3}$ 448.8892 (100\%), 450.8862 (64\%), 446.8921 (63\%), 452.8833 (20\%), 449.8925 (20\%); found 448.8878 (100\%), 450.8852 (66\%), 446.8909 (65\%), 452.8826 (19\%), 449.8913 (19\%).

\section{3,3',5,5'-Tetrachloro-2'-(2,4-dichlorophenoxy)-[1,1'-biphenyl]-2,4'-diol - Ambigol E (5)}
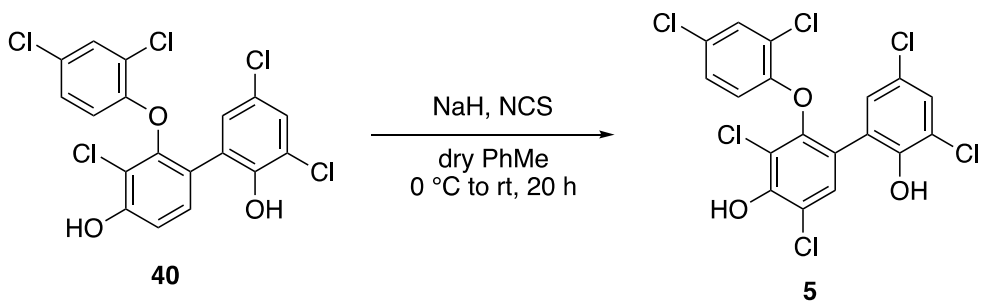

Compound 40 ( $38.0 \mathrm{mg}, 84.3 \mu \mathrm{mol}, 1.0$ eq.) was dissolved in dry toluene ( $2.0 \mathrm{~mL}$ ) and cooled to $0{ }^{\circ} \mathrm{C}$. Sodium hydride (60\% dispersion, $\left.6.74 \mathrm{mg}, 169 \mu \mathrm{mol}, 2.0 \mathrm{eq}\right)$ and NCS (11.3 mg, 84.3 $\mu \mathrm{mol}, 1.0$ eq.) were slowly added. The reaction mixture was allowed to warm to room temperature and stirred for $20 \mathrm{~h}$. After dilution with $1 \mathrm{~N} \mathrm{HCl}(2.0 \mathrm{~mL})$, the obtained layers were separated, and the aqueous phase was extracted with $\mathrm{Et}_{2} \mathrm{O}(2 \mathrm{x})$. The combined organic layers were washed with brine, dried over $\mathrm{Na}_{2} \mathrm{SO}_{4}$ and filtered. The solvent was removed in vacuo. The crude material was purified by MPLC to give the desired product $(5,21.0 \mathrm{mg}, 43.3 \mu \mathrm{mol}$, $51 \%)$ as a white solid. Analytical data were in agreement with literature values. ${ }^{13}$ 
${ }^{1} \mathrm{H}-\mathrm{NMR}\left(300 \mathrm{MHz}, \mathrm{CDCl}_{3}\right): \delta=7.38(\mathrm{~s}, 1 \mathrm{H}), 7.28-7.23(\mathrm{~m}, 2 \mathrm{H}), 7.09(\mathrm{~d}, J=2.5 \mathrm{~Hz}, 1 \mathrm{H}), 6.97$ (dd, J=8.8, $2.5 \mathrm{~Hz}, 1 \mathrm{H}$ ), 6.41 (d, $J=8.8 \mathrm{~Hz}, 1 \mathrm{H}$ ), 6.09 (bs, $1 \mathrm{H}), 5.57$ (bs, $1 \mathrm{H}$ ). ${ }^{13} \mathrm{C}-\mathrm{NMR}$ $\left(75 \mathrm{MHz}, \mathrm{CDCl}_{3}\right): \delta=151.3,149.3,147.8,147.7,130.3,130.1,128.8,127.95,127.94,127.4$, $125.4,124.1,123.5,123.4,121.2,118.0,116.3,115.8 .{ }^{1} \mathrm{H}-\mathrm{NMR}\left(300 \mathrm{MHz}, \mathrm{MeOD}-\mathrm{d}_{4}\right): \delta=7.34$ $(\mathrm{s}, 1 \mathrm{H}), 7.29$ (d, J = 2.6 Hz, $1 \mathrm{H}), 7.22(\mathrm{~d}, J=2.6 \mathrm{~Hz}, 1 \mathrm{H}), 7.05$ (dd, J = 8.9, $2.5 \mathrm{~Hz}, 1 \mathrm{H}), 6.99$ (d, $J=2.6 \mathrm{~Hz}, 1 \mathrm{H}$ ), 6.50 (d, $J=8.8 \mathrm{~Hz}, 1 \mathrm{H}$ ). ${ }^{13} \mathrm{C}-\mathrm{NMR}\left(75 \mathrm{MHz}, \mathrm{MeOD}-\mathrm{d}_{4}\right): \delta=152.9,152.1,150.8$, 149.1, 131.4, 130.8, 130.7, 129.9, 128.55, 128.48, 126.9, 125.0, 124.26, 124.25, 123.0, 120.0, 118.1, 117.4. MS (ESI-): $\mathrm{m} / \mathrm{z}=480.8[\mathrm{M}-\mathrm{H}]^{-}$. HRMS (ESI-TOF) $\mathrm{m} / \mathrm{z}:[\mathrm{M}+\mathrm{Na}]^{+}$calcd for $\mathrm{C}_{18} \mathrm{H}_{8} \mathrm{Cl}_{6} \mathrm{NaO}_{3} 506.8473$ (100\%), 508.8443 (80\%), 504.8502 (52\%), 510.8414 (34\%); found $506.8461(100 \%), 508.8434(80 \%), 504.8484$ (51\%), $510.8409(33 \%)$. HPLC: $t_{R}=17.06 \mathrm{~min}$.

\subsection{Synthesis of Structural Analogue 47}

\section{2-Methoxyphenyl acetate (42)}

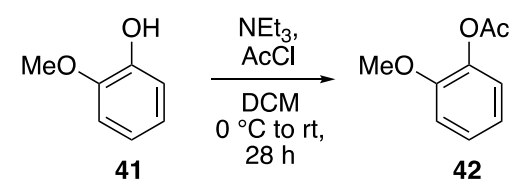

Guaiacol (41, $1.80 \mathrm{~mL}, 2.00 \mathrm{~g}, 16.1 \mathrm{mmol}, 1.0$ eq.) was dissolved in $\mathrm{DCM}(64.4 \mathrm{~mL}$, $4.0 \mathrm{~mL} / \mathrm{mmol}$ ) and cooled to $0{ }^{\circ} \mathrm{C}$. Triethylamine $(2.69 \mathrm{~mL}, 1.95 \mathrm{~g}, 19.3 \mathrm{mmol}, 1.2 \mathrm{eq}$.$) and$ freshly distilled acetyl chloride $(2.87 \mathrm{~mL}, 3.16 \mathrm{~g}, 40.3 \mathrm{mmol}, 2.5 \mathrm{eq}$.) were added to the reaction mixture before being allowed to warm up to room temperature. The mixture was stirred for further $28 \mathrm{~h}$. The reaction solution was evaporated under reduced pressure and treated with $\mathrm{DCM} / \mathrm{H}_{2} \mathrm{O}$. The obtained layers were separated, and the aqueous phase was extracted with DCM (3x). The combined organic layers were washed with brine, dried over $\mathrm{Na}_{2} \mathrm{SO}_{4}$ and filtered. The solvent was removed in vacuo to give the desired product $(42,2.68 \mathrm{~g}$, $16.1 \mathrm{mmol}, 99 \%)$ as a white solid. Analytical data were in agreement with literature values. ${ }^{15}$

${ }^{1} \mathrm{H}-\mathrm{NMR}\left(300 \mathrm{MHz}, \mathrm{CDCl}_{3}\right): \delta=7.21(\mathrm{ddd}, J=8.3,7.3,1.8 \mathrm{~Hz}, 1 \mathrm{H}), 7.05(\mathrm{dd}, J=7.8,1.7 \mathrm{~Hz}, 1 \mathrm{H})$, 7.00-6.91 (m, $2 \mathrm{H}), 3.83(\mathrm{~s}, 3 \mathrm{H}), 2.32(\mathrm{~s}, 3 \mathrm{H}) .{ }^{13} \mathrm{C}-\mathrm{NMR}\left(75 \mathrm{MHz}, \mathrm{CDCl}_{3}\right): \delta=169.1,151.2$, $139.8,127.0,122.9,120.8,112.5,55.9,20.7 . \mathrm{MS}(\mathrm{ESI}+): \mathrm{m} / \mathrm{z}=333.0[2 \mathrm{M}+\mathrm{H}]^{+}, 210.8[\mathrm{M}+2 \mathrm{Na}-$ $\mathrm{H}]^{+}, 189.1[\mathrm{M}+\mathrm{Na}]^{+}, 167.3[\mathrm{M}+\mathrm{H}]^{+}$.

\section{3,5-Dichloro-2-methoxyphenyl acetate (43)}

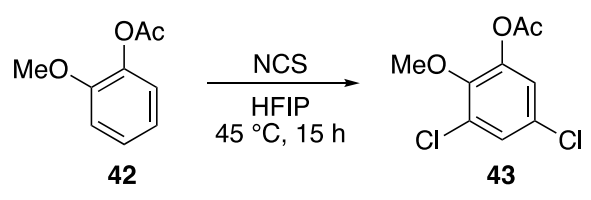

The chlorination was conducted according to a published procedure and similar to compound 29. ${ }^{12}$ The product 43 was isolated as a yellow oil (486 mg, $2.07 \mathrm{mmol}, 69 \%$ ). 
${ }^{1} \mathrm{H}-\mathrm{NMR}\left(300 \mathrm{MHz}, \mathrm{CDCl}_{3}\right): \delta=7.27(\mathrm{~d}, J=2.5 \mathrm{~Hz}, 1 \mathrm{H}), 7.02(\mathrm{~d}, J=2.5 \mathrm{~Hz}, 1 \mathrm{H}), 3.84(\mathrm{~s}, 3 \mathrm{H})$, 2.33 (s, $3 \mathrm{H}$ ). ${ }^{13} \mathrm{C}-\mathrm{NMR}\left(75 \mathrm{MHz}, \mathrm{CDCl}_{3}\right): \delta=168.6,147.6,145.1,129.5,129.0,127.9,122.7$, 61.3, 20.7. MS (ESI+): $\mathrm{m} / \mathrm{z}=506.7[2 \mathrm{M}+\mathrm{K}]^{+}, 490.8[2 \mathrm{M}+\mathrm{Na}]^{+}, 468.9[2 \mathrm{M}+\mathrm{H}]^{+}, 279.0[\mathrm{M}+2 \mathrm{Na}-$ $\mathrm{H}^{+}, 273.2[\mathrm{M}+\mathrm{K}]^{+}, 257.2[\mathrm{M}+\mathrm{Na}]^{+}, 235.2[\mathrm{M}+\mathrm{H}]^{+}$. HRMS (ESI-TOF) $\mathrm{m} / \mathrm{z}:[\mathrm{M}+\mathrm{Na}]^{+}$calcd for $\mathrm{C}_{9} \mathrm{H}_{8} \mathrm{Cl}_{2} \mathrm{NaO}_{3} 256.9748$ (100\%), 258.9719 (64\%), 260.9689 (10\%), 257.9782 (10\%); found 256.9743 (100\%), 258.9717 (60\%), 260.9688 (9\%), 257.9777 (9\%).

\section{3,5-Dichloro-2-methoxyphenol (44)}<smiles>COc1cc(Cl)cc(Cl)c1OC(C)=O</smiles>

43

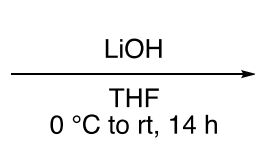

$0^{\circ} \mathrm{C}$ to rt, $14 \mathrm{~h}$<smiles>COc1c(O)cc(Cl)cc1O</smiles>

44

Compound 43 ( $486 \mathrm{mg}, 2.07 \mathrm{mmol}, 1.0$ eq.) was dissolved in THF (6.3 mL, $2.0 \mathrm{~mL} / \mathrm{mmol}$ ) and cooled to $0{ }^{\circ} \mathrm{C}$. $0.1 \mathrm{~N} \mathrm{LiOH}$ solution $(6.21 \mathrm{~mL}, 6.21 \mathrm{mmol}, 3.0$ eq.) was added dropwise to the reaction mixture. The reaction was stirred at room temperature for $14 \mathrm{~h}$. The organic solvent was removed in vacuo and the remaining aqueous phase was extracted with DCM (2x), acidified to $\mathrm{pH}=1$ with aqueous $10 \% \mathrm{KHSO}_{4}$ solution and repeatedly extracted with $\mathrm{DCM}(2 \times)$. The combined organic layers were washed with brine, dried over $\mathrm{Na}_{2} \mathrm{SO}_{4}$ and filtered. The solvent was removed in vacuo to give the desired product $(\mathbf{4 4}, 367 \mathrm{mg}, 1.90 \mathrm{mmol}, 92 \%)$ as yellow solid.

${ }^{1} \mathrm{H}-\mathrm{NMR}\left(300 \mathrm{MHz}, \mathrm{CDCl}_{3}\right): \delta=6.91(\mathrm{~d}, J=2.4 \mathrm{~Hz}, 1 \mathrm{H}), 6.89(\mathrm{~d}, J=2.5 \mathrm{~Hz}, 1 \mathrm{H}), 5.81$ (bs, $1 \mathrm{H}$ ), 3.91 (s, $3 \mathrm{H}$ ). ${ }^{13} \mathrm{C}-\mathrm{NMR}\left(75 \mathrm{MHz}, \mathrm{CDCl}_{3}\right): \delta=150.5,142.4,130.0,127.5,121.6,115.0,61.4 . \mathrm{MS}$ $(\mathrm{ESI}+): \mathrm{m} / \mathrm{z}=407.2[2 \mathrm{M}+\mathrm{Na}]^{+}, 214.9[\mathrm{M}+\mathrm{Na}]^{+}$. HRMS (ESI-TOF) $\mathrm{m} / \mathrm{z}:[\mathrm{M}-\mathrm{H}]^{-}$calcd for $\mathrm{C}_{7} \mathrm{H}_{5} \mathrm{Cl}_{2} \mathrm{O}_{2}$ 190.9672 (100\%), 192.9642 (64\%), 194.9613 (10\%); found 190.9664 (100\%), 192.9638 (63\%), 194.9609 (9\%).

\section{1,5-Dichloro-3-(2,4-dichlorophenoxy)-2-iodo-4-methoxybenzene (45)}<smiles>COc1c(O)cc(Cl)cc1Cl</smiles>

44

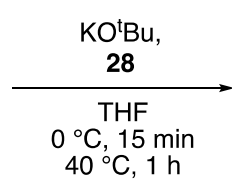

$0^{\circ} \mathrm{C}, 15 \min$
$40^{\circ} \mathrm{C}, 1 \mathrm{~h}$

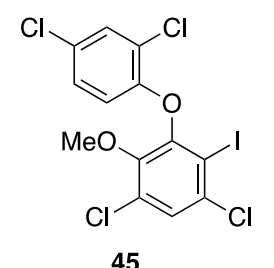

45

Compound $\mathbf{4 5}$ was synthesized following the general procedure in section 2.1 and isolated as a white solid (100 mg, $0.22 \mathrm{mmol}, 46 \%$ ).

${ }^{1} \mathrm{H}-\mathrm{NMR}\left(300 \mathrm{MHz}, \mathrm{CDCl}_{3}\right): \delta=7.49(\mathrm{~s}, 1 \mathrm{H}), 7.48(\mathrm{~d}, J=2.5 \mathrm{~Hz}, 1 \mathrm{H}), 7.07$ (dd, J=8.8, $2.5 \mathrm{~Hz}$, $1 \mathrm{H}), 6.40(\mathrm{~d}, J=8.8 \mathrm{~Hz}, 1 \mathrm{H}), 3.81(\mathrm{~s}, 3 \mathrm{H}) .{ }^{13} \mathrm{C}-\mathrm{NMR}\left(75 \mathrm{MHz}, \mathrm{CDCl}_{3}\right): \delta=151.0,150.1,147.9$, $134.9,130.6,130.0,128.2,127.8,127.3,123.5,115.6,96.0,61.8 . \mathrm{MS}(\mathrm{ESI}+): \mathrm{m} / \mathrm{z}=946.3$ $[2 \mathrm{M}+\mathrm{Na}]^{+}, 506.7[\mathrm{M}+2 \mathrm{Na}-\mathrm{H}]^{+}, 484.7[\mathrm{M}+\mathrm{Na}]^{+}, 462.8[\mathrm{M}+\mathrm{H}]^{+} . \mathrm{MS}(\mathrm{ESI}-): \mathrm{m} / \mathrm{z}=497.0[\mathrm{M}+\mathrm{Cl}]^{-}$, $482.7[\mathrm{M}+\mathrm{Na}-2 \mathrm{H}]^{-}, 461.0[\mathrm{M}-\mathrm{H}]^{-}$. HRMS (ESI-TOF) $\mathrm{m} / \mathrm{z}$ : $[\mathrm{M}+\mathrm{Na}]^{+}$calcd for $\mathrm{C}_{13} \mathrm{H}_{7} \mathrm{Cl}_{4} \mathrm{INaO}_{2}$ 
486.8113 (100\%), 484.8143 (78\%), 488.8084 (48\%), 487.8147 (14\%), 485.8176 (11\%); found 486.8101 (100\%), 484.8130 (77\%), 488.8073 (50\%), 487.8135 (13\%), 485.8158 (12\%).

\section{3',4,5',6-Tetrachloro-2-(2,4-dichlorophenoxy)-2',3-dimethoxy-1,1'-biphenyl (46)}

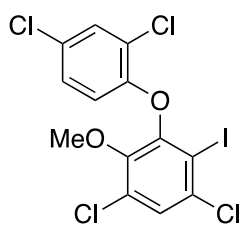

45
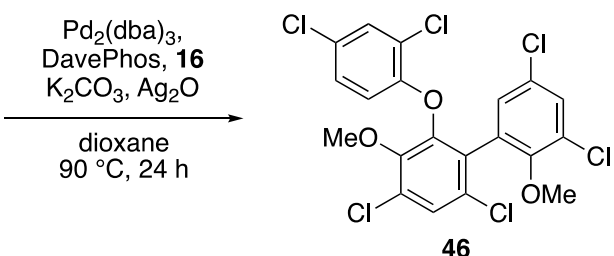

46

The Suzuki coupling was performed according to the general procedure in section 2.1. The product 46 was obtained in $20 \%$ yield $(12.6 \mathrm{mg}, 24.6 \mu \mathrm{mol})$ as a yellowish solid. The substrate $(45,4.64 \mathrm{mg}, 10.0 \mu \mathrm{mol})$ was re-isolated in $8 \%$ yield, based on which the desired product 46 was obtained in $28 \%$ isolated yield.

${ }^{1} \mathrm{H}-\mathrm{NMR}\left(300 \mathrm{MHz}, \mathrm{CDCl}_{3}\right): \delta=7.47(\mathrm{~s}, 1 \mathrm{H}), 7.37(\mathrm{~d}, J=2.5 \mathrm{~Hz}, 1 \mathrm{H}), 7.29(\mathrm{~d}, J=2.5 \mathrm{~Hz}, 1 \mathrm{H})$, $7.06(\mathrm{dd}, J=8.8,2.5 \mathrm{~Hz}, 1 \mathrm{H}), 6.84(\mathrm{~d}, J=2.6 \mathrm{~Hz}, 1 \mathrm{H}), 6.55(\mathrm{~d}, J=8.8 \mathrm{~Hz}, 1 \mathrm{H}), 3.82(\mathrm{~s}, 3 \mathrm{H}), 3.71$ (s, $3 \mathrm{H}) .{ }^{13} \mathrm{C}-\mathrm{NMR}\left(75 \mathrm{MHz}, \mathrm{CDCl}_{3}\right): \delta=153.1,151.9,148.4,147.4,131.0,130.3,130.2,129.7$, 129.6, 129.4, 129.0, 128.9 128.1, 127.66, 127.65, 123.4, 116.7, 61.7, 61.4. One carbon signal missing due to overlap. MS $(\mathrm{ESI}+): \mathrm{m} / \mathrm{z}=1043.0[2 \mathrm{M}+\mathrm{Na}]^{+}, 549.0[\mathrm{M}+\mathrm{K}]^{+}, 533.1[\mathrm{M}+\mathrm{Na}]^{+}$. HRMS (ESI-TOF) $\mathrm{m} / \mathrm{z}$ : [M+Na] ${ }^{+}$calcd for $\mathrm{C}_{20} \mathrm{H}_{12} \mathrm{Cl}_{6} \mathrm{NaO}_{3} 534.8781$ (100\%), 536.8751 (80\%), 532.8810 (52\%), 538.8722 (34\%); found 534.8782 (100\%), 536.8750 (82\%), 532.8843 (55\%), 538.8723 (35\%).

\section{3,4',5,6'-Tetrachloro-2'-(2,4-dichlorophenoxy)-[1,1'-biphenyl]-2,3'-diol (47)}

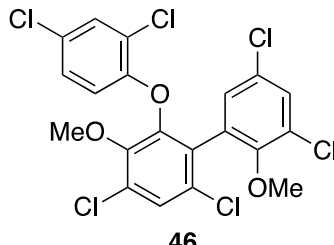

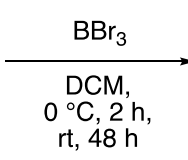

$\mathrm{rt}, 48 \mathrm{~h}$

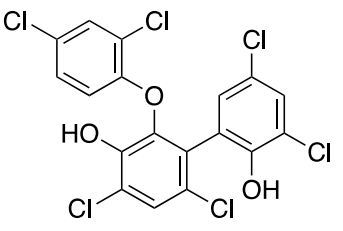

47

Compound $\mathbf{4 7}$ was synthesized following the general procedure in section 2.1 and isolated as a white solid (17.0 mg, $35.1 \mu \mathrm{mol}, 99 \%$ ).

${ }^{1} \mathrm{H}-\mathrm{NMR}\left(300 \mathrm{MHz}, \mathrm{CDCl}_{3}\right): \delta=7.42(\mathrm{~s}, 1 \mathrm{H}), 7.26-7.25(\mathrm{~m}, 1 \mathrm{H}), 7.20(\mathrm{~d}, J=2.5 \mathrm{~Hz}, 1 \mathrm{H}), 6.99$ (dd, $J=8.8,2.5 \mathrm{~Hz}, 1 \mathrm{H}), 6.90(\mathrm{~d}, J=2.5 \mathrm{~Hz}, 1 \mathrm{H}), 6.56(\mathrm{~d}, J=8.8 \mathrm{~Hz}, 1 \mathrm{H}), 5.92(\mathrm{bs}, 1 \mathrm{H}), 5.46$ (bs, $1 \mathrm{H}$ ). ${ }^{13} \mathrm{C}-\mathrm{NMR}\left(75 \mathrm{MHz}, \mathrm{CDCl}_{3}\right): \delta=151.3,147.7,144.6,141.6,130.1,130.0,129.1,128.6$, $128.2,127.4,126.9,125.9,125.3,124.0,122.3,121.9,120.9,117.4$. MS (ESI-): $\mathrm{m} / \mathrm{z}=480.9$ [M-H] $]^{-}$. HRMS (ESI-TOF) $\mathrm{m} / \mathrm{z}$ : [M+Na] ${ }^{+}$calcd for $\mathrm{C}_{18} \mathrm{H}_{8} \mathrm{Cl}_{6} \mathrm{NaO}_{3} 506.8473$ (100\%), 508.8443 (80\%), 504.8502 (52\%), 510.8414 (34\%); found 506.8461 (100\%), 508.8435 (77\%), 504.8484 (49\%), 510.8408 (34\%). HPLC: $t_{R}=18.83 \mathrm{~min}$. 


\section{NMR Data}

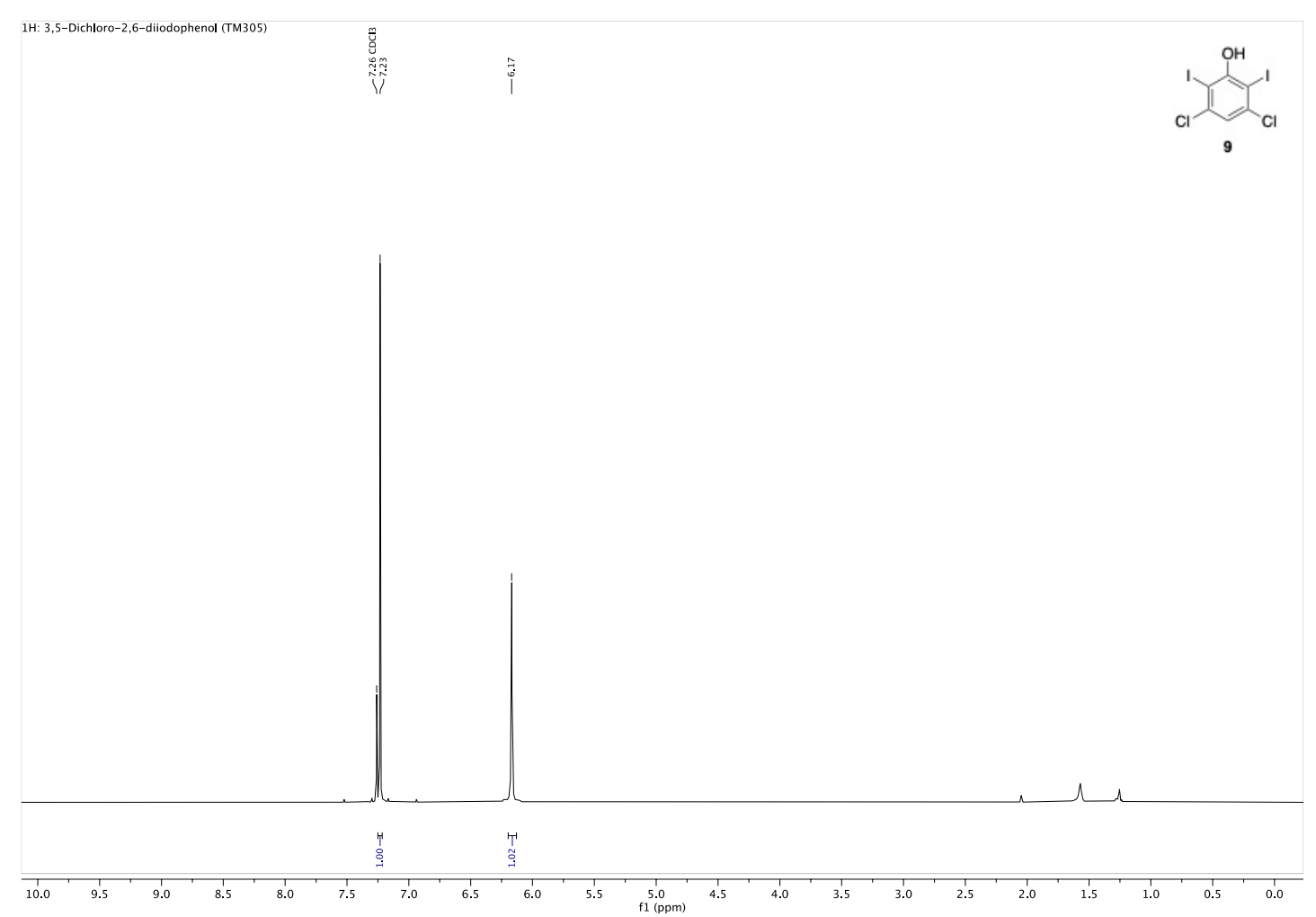

Fig. S2 ${ }^{1} \mathrm{H}-\mathrm{NMR}$ spectrum of 3,5-dichloro-2,6-diiodophenol (9), measured in $\mathrm{CDCl}_{3}$ at $300 \mathrm{MHz}$.

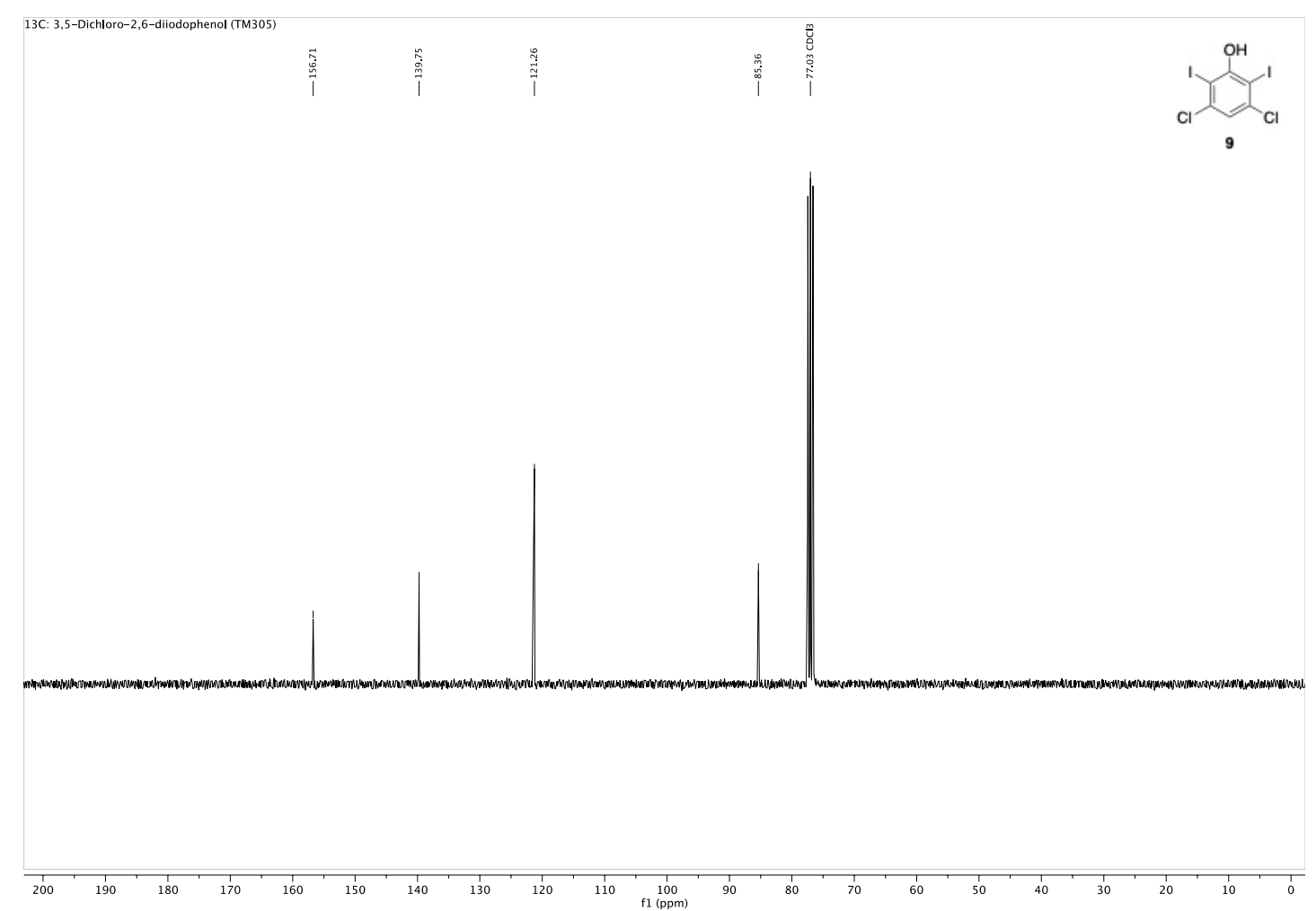

Fig. S3 ${ }^{13} \mathrm{C}$-NMR spectrum of 3,5-dichloro-2,6-diiodophenol (9), measured in $\mathrm{CDCl}_{3}$ at $75 \mathrm{MHz}$. 


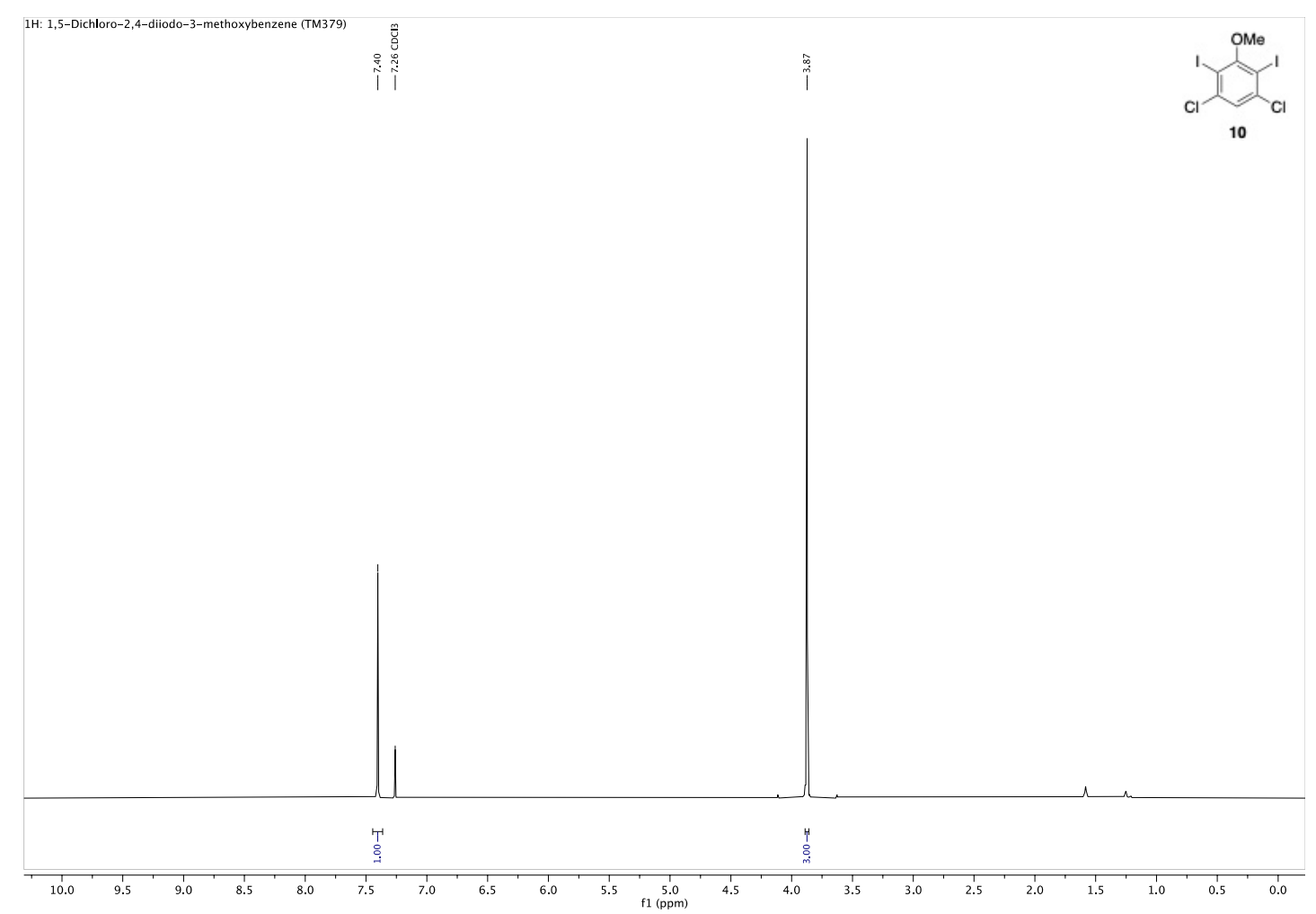

Fig. S4 ${ }^{1} \mathrm{H}-\mathrm{NMR}$ spectrum of 1,5-dichloro-2,4diiodo-3-methoxybenzene (10), measured in $\mathrm{CDCl}_{3}$ at $300 \mathrm{MHz}$.

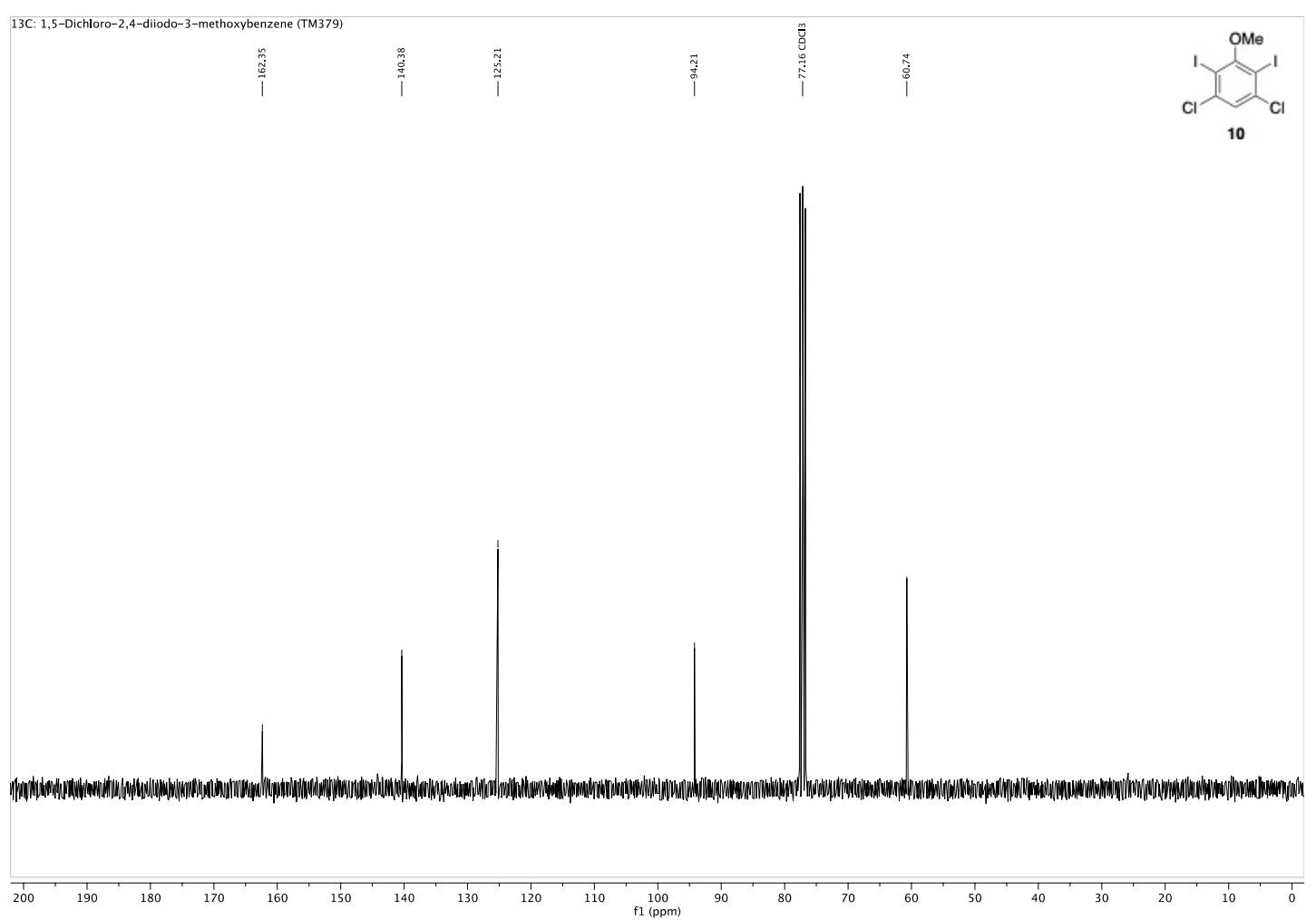

Fig. S5 ${ }^{13} \mathrm{C}-\mathrm{NMR}$ spectrum of 1,5-dichloro-2,4diiodo-3-methoxybenzene (10), measured in $\mathrm{CDCl}_{3}$ at $75 \mathrm{MHz}$. 


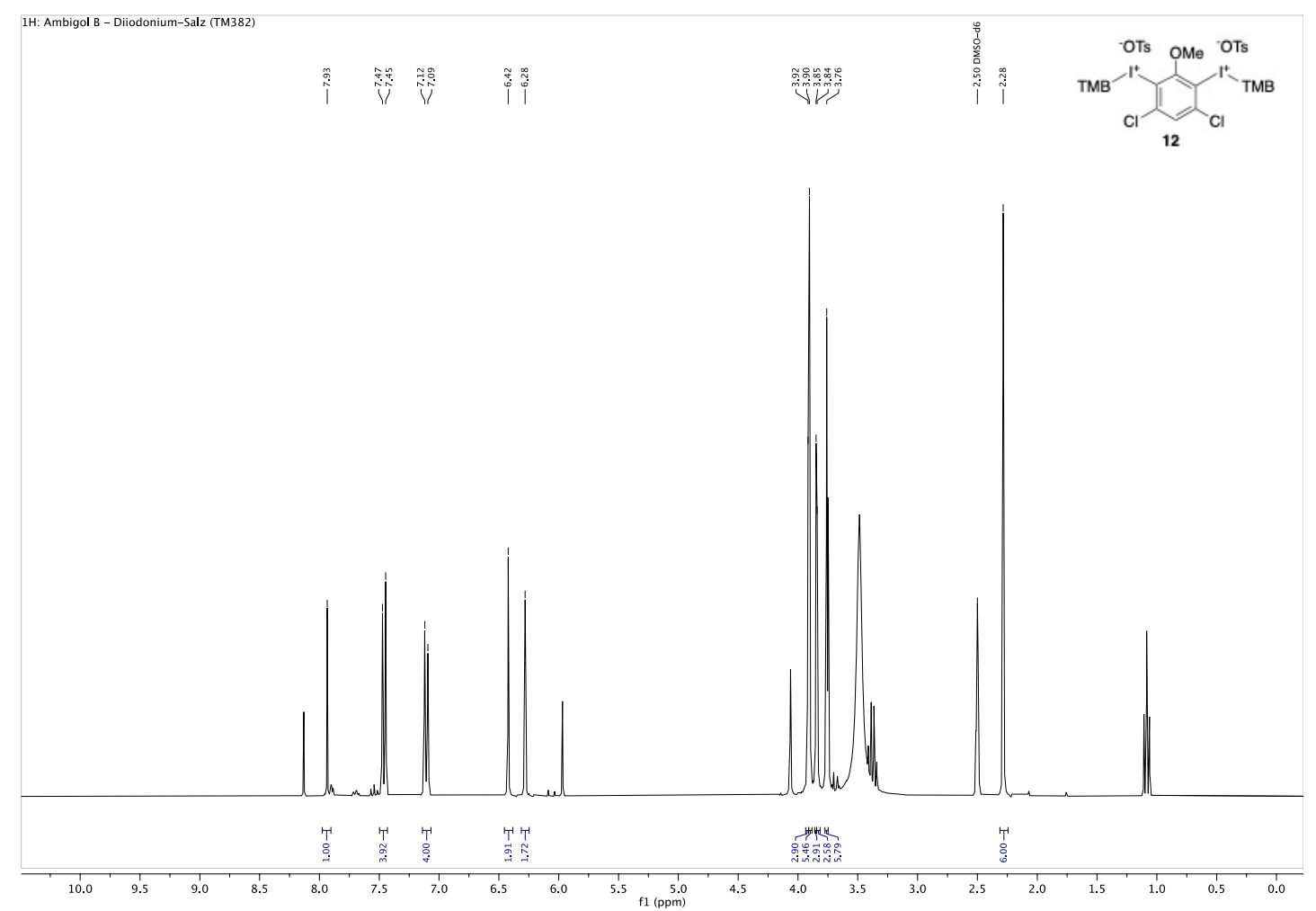

Fig. S6 ${ }^{1} \mathrm{H}-\mathrm{NMR}$ spectrum of (4,6-dichloro-2-methoxy-1,3-phenylene)bis((2,4,6-trimethoxyphenyl)iodonium) bistosylat (12), measured in DMSO-d $\mathrm{d}_{6}$ at $600 \mathrm{MHz}$. 


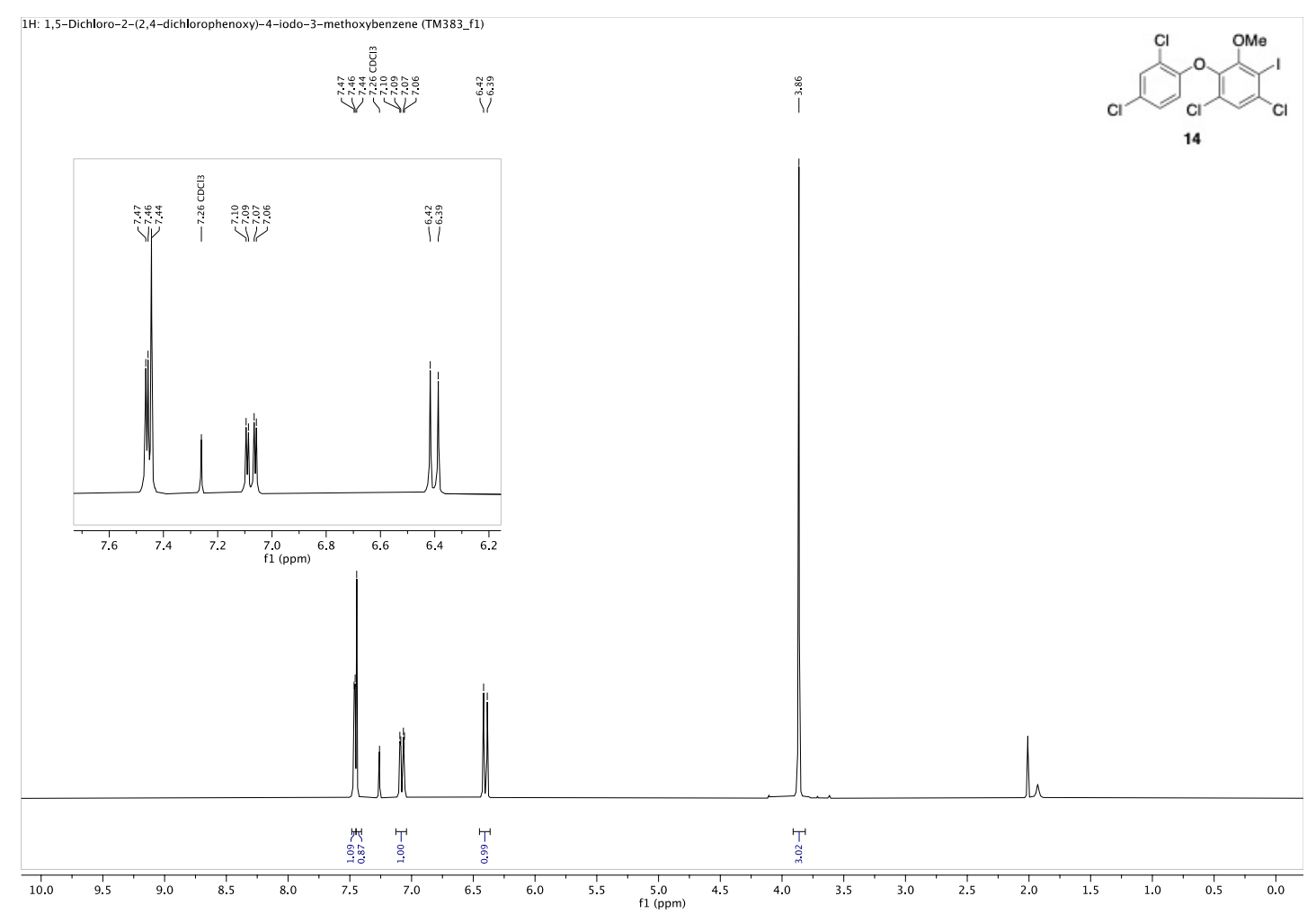

Fig. S7 ${ }^{1} \mathrm{H}-\mathrm{NMR}$ spectrum of 1,5-dichloro-2-(2,4-dichlorophenoxy)-4-iodo-3-methoxybenzene (14), measured in $\mathrm{CDCl}_{3}$ at $300 \mathrm{MHz}$.

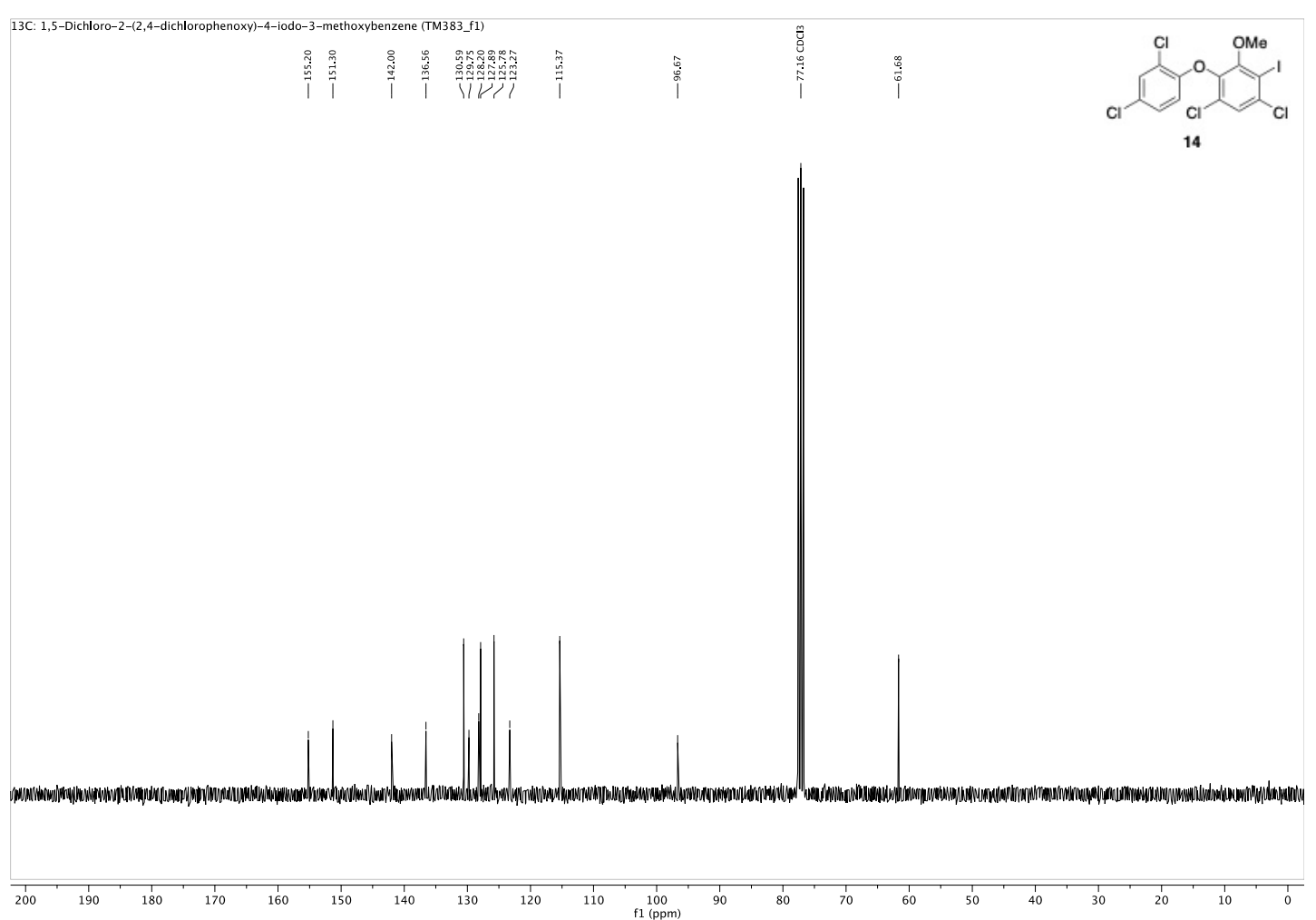

Fig. S8 ${ }^{13} \mathrm{C}-\mathrm{NMR}$ spectrum of 1,5-dichloro-2-(2,4-dichlorophenoxy)-4-iodo-3-methoxybenzene (14), measured in $\mathrm{CDCl}_{3}$ at $75 \mathrm{MHz}$. 


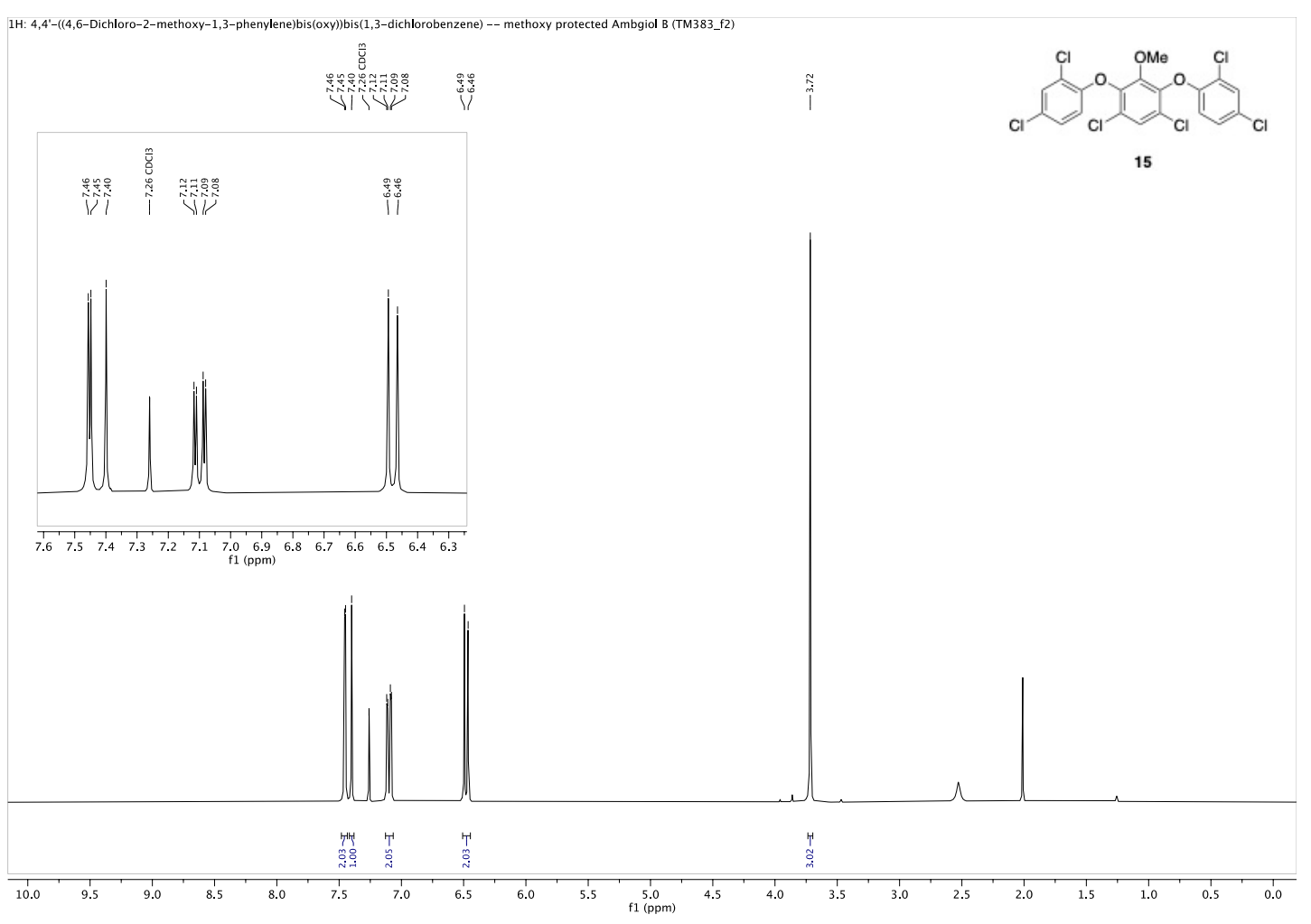

Fig. S9 ${ }^{1} \mathrm{H}-\mathrm{NMR}$ spectrum of 4,4'-((4,6-dichloro-2-methoxy-1,3-phenylene)bis(oxy))bis(1,3-dichlorobenzene) (15), measured in $\mathrm{CDCl}_{3}$ at $300 \mathrm{MHz}$.

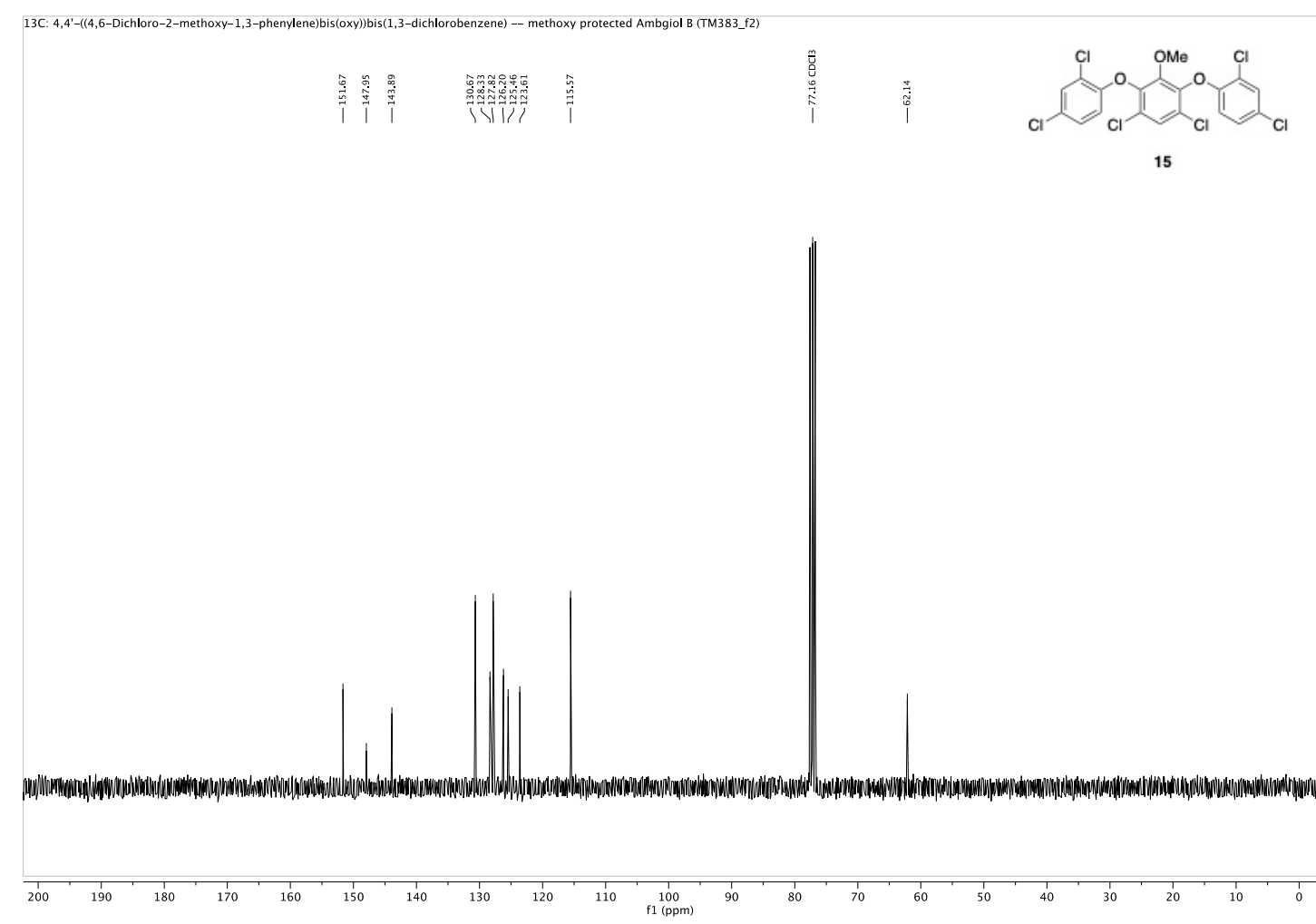

Fig. S10 ${ }^{13}$ C-NMR spectrum of 4,4'-((4,6-dichloro-2-methoxy-1,3-phenylene)bis(oxy))bis(1,3-dichlorobenzene) (15), measured in $\mathrm{CDCl}_{3}$ at $75 \mathrm{MHz}$. 


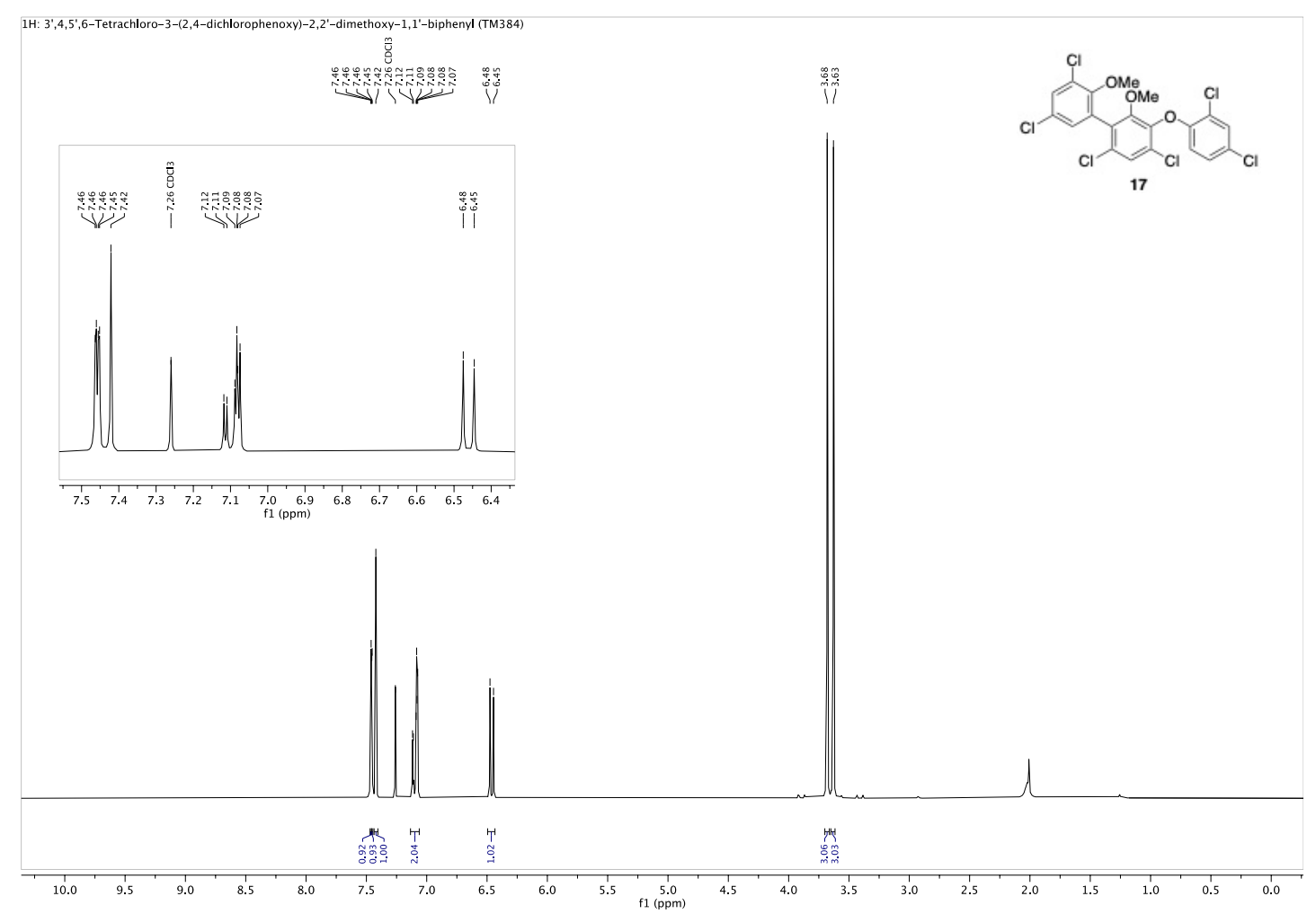

Fig. S11 ${ }^{1} \mathrm{H}$-NMR spectrum of 3',4,5',6-tetrachloro-3-(2,4-dichlorophenoxy)-2,2'-dimethoxy-1,1'-biphenyl (17), measured in $\mathrm{CDCl}_{3}$ at $300 \mathrm{MHz}$.

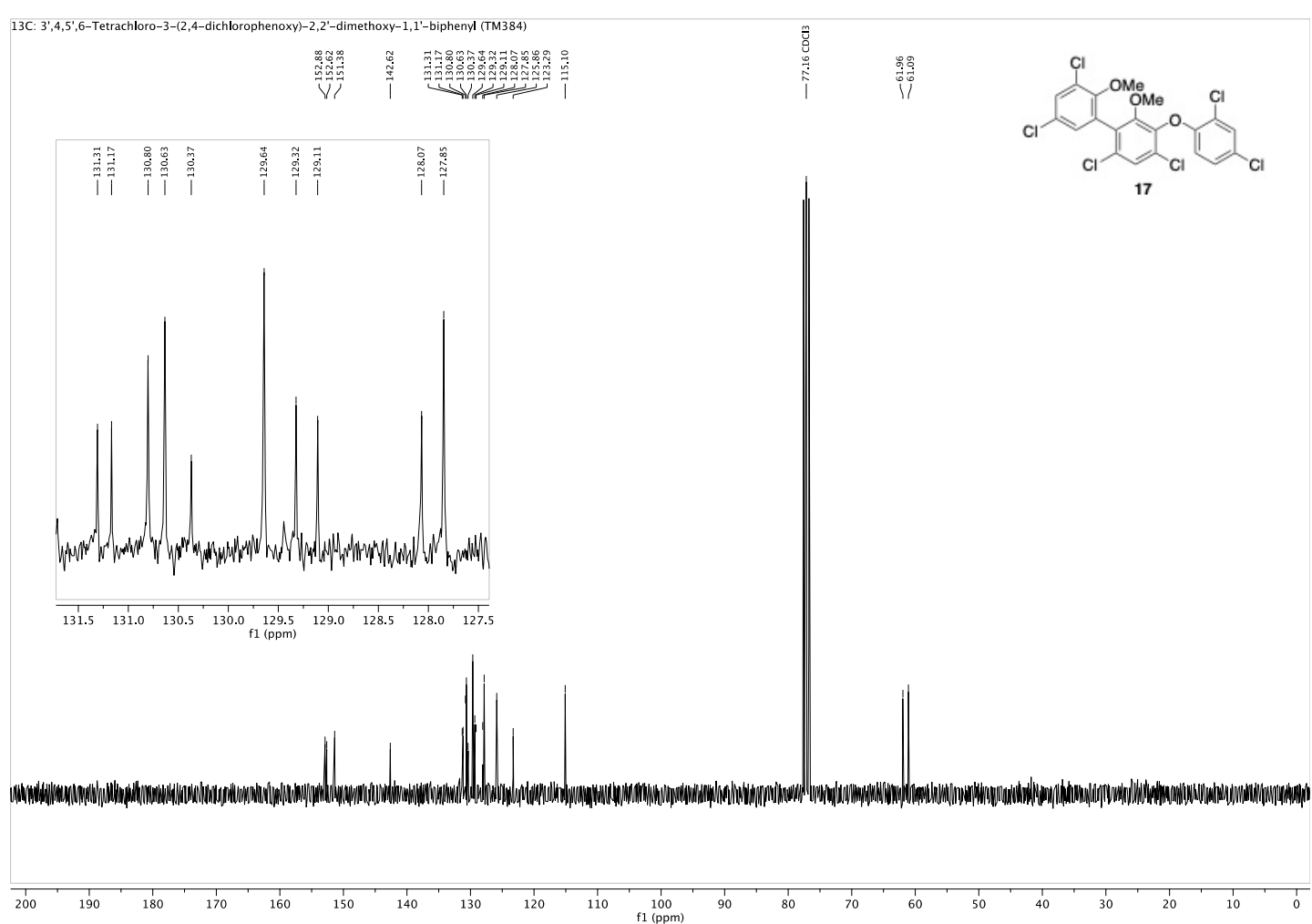

Fig. S12 ${ }^{13}$ C-NMR spectrum of 3',4,5',6-tetrachloro-3-(2,4-dichlorophenoxy)-2,2'-dimethoxy-1,1'-biphenyl (17), measured in $\mathrm{CDCl}_{3}$ at $75 \mathrm{MHz}$. 


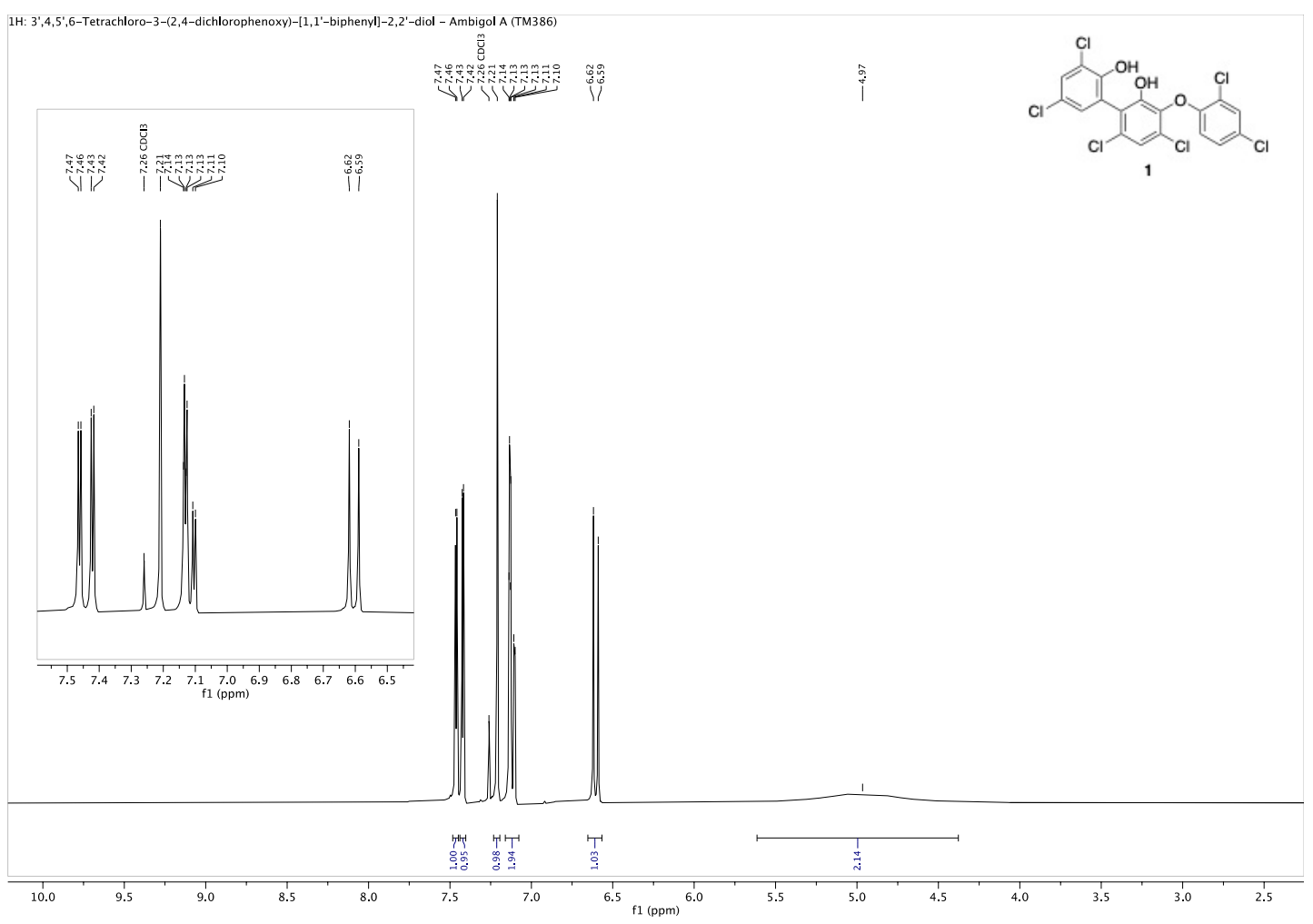

Fig. S13 ${ }^{1} \mathrm{H}-\mathrm{NMR}$ spectrum of ambigol $\mathrm{A}(\mathbf{1})$, measured in $\mathrm{CDCl}_{3}$ at $300 \mathrm{MHz}$.

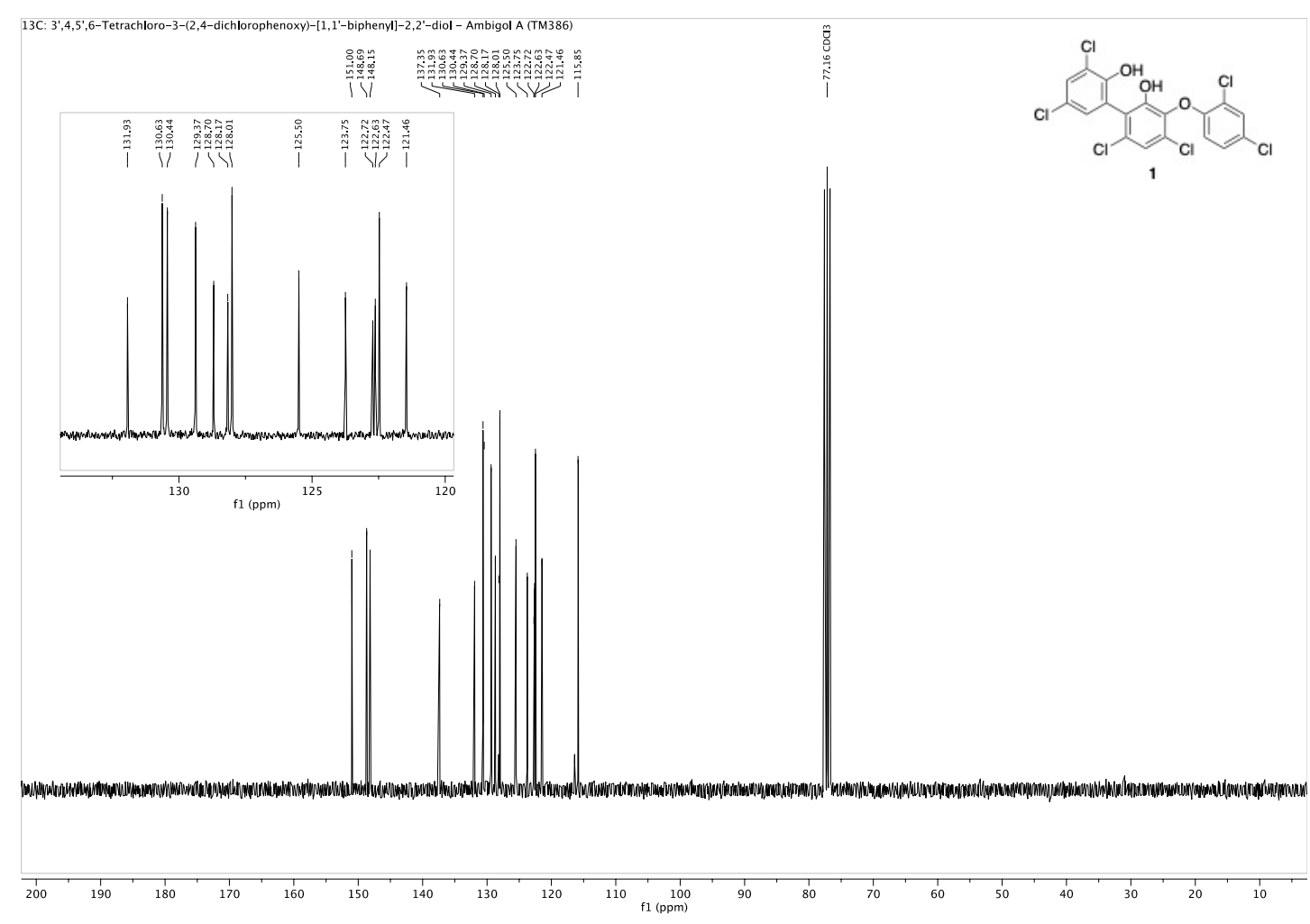

Fig. S14 ${ }^{13} \mathrm{C}-\mathrm{NMR}$ spectrum of ambigol $\mathrm{A}(1)$, measured in $\mathrm{CDCl}_{3}$ at $75 \mathrm{MHz}$. 


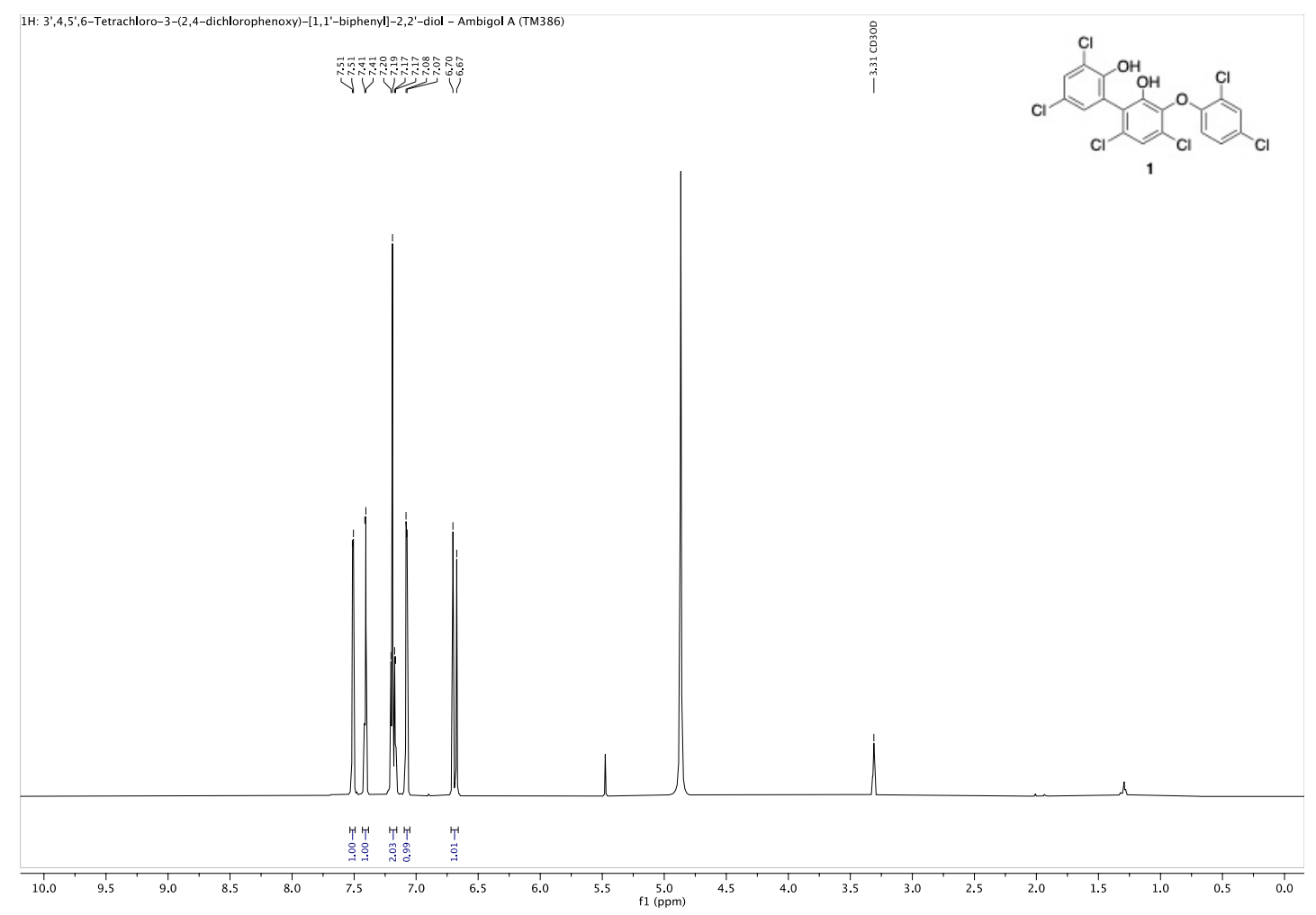

Fig. S15 ${ }^{1} \mathrm{H}-\mathrm{NMR}$ spectrum of ambigol A (1), measured in MeOD-d 4 at $300 \mathrm{MHz}$.

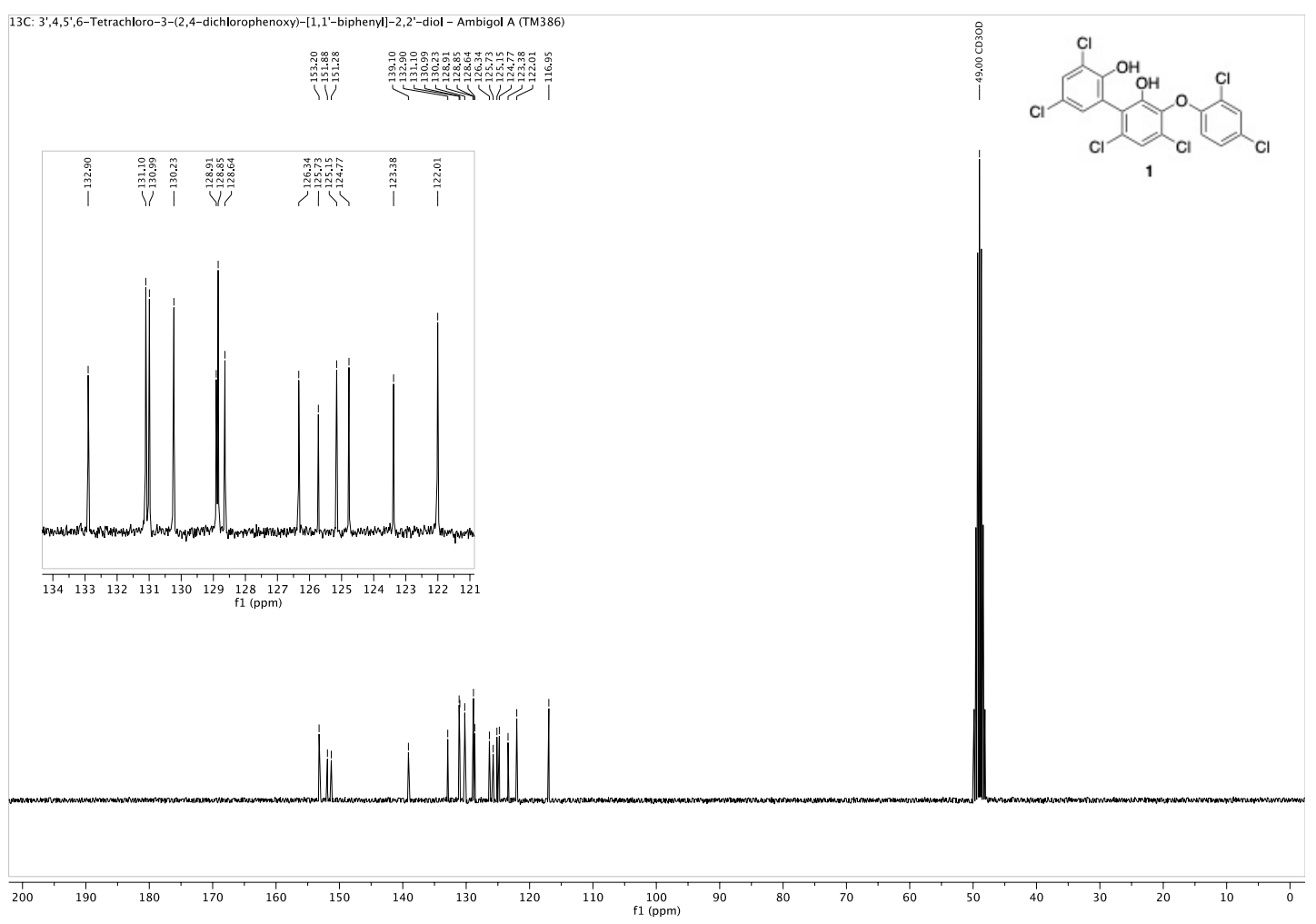

Fig. S16 ${ }^{13} \mathrm{C}-\mathrm{NMR}$ spectrum of ambigol A (1), measured in MeOD-d 4 at $75 \mathrm{MHz}$. 


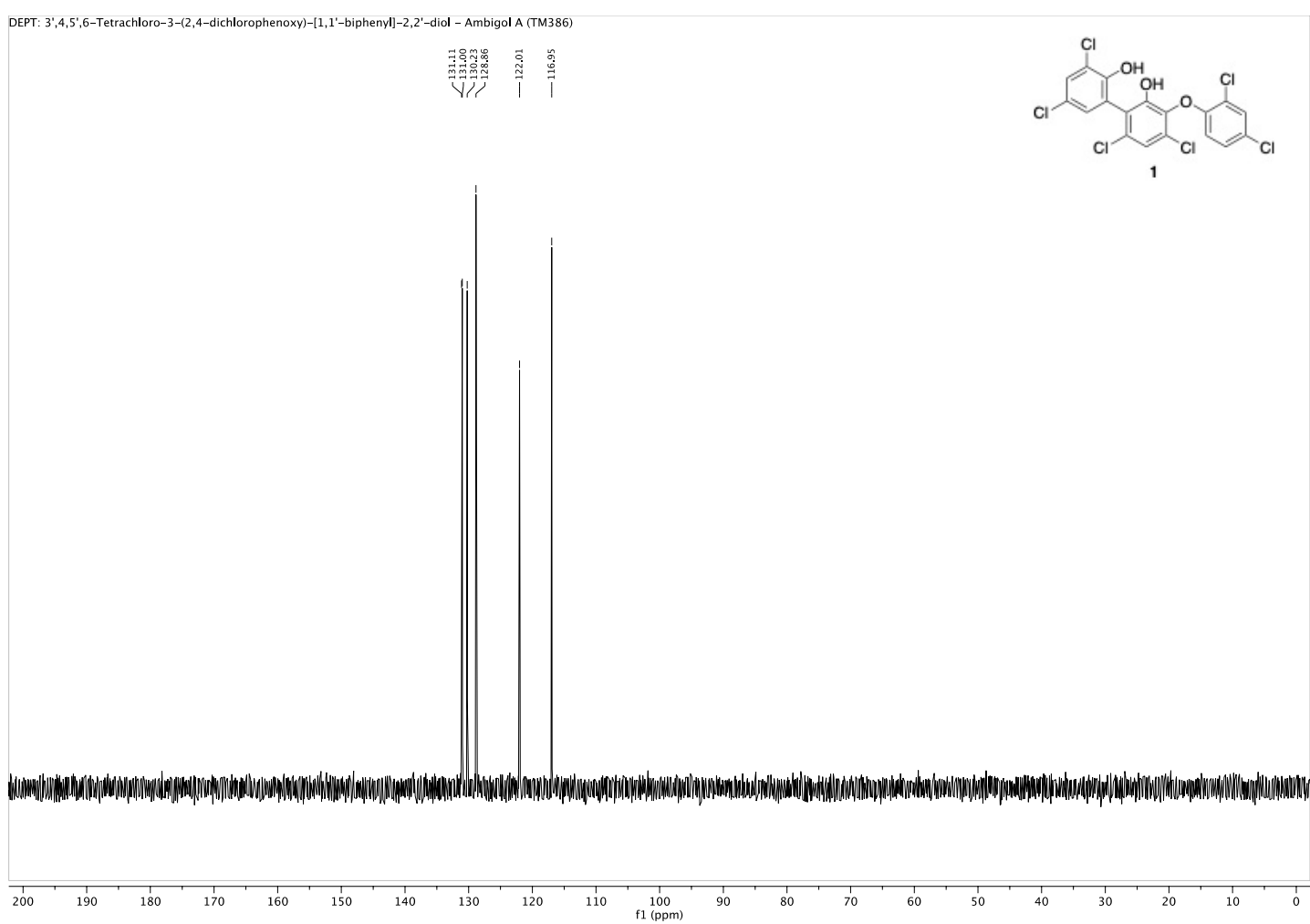

Fig. S17 DEPT NMR spectrum of ambigol A (1), measured in MeOD-d 4 at $75 \mathrm{MHz}$. 


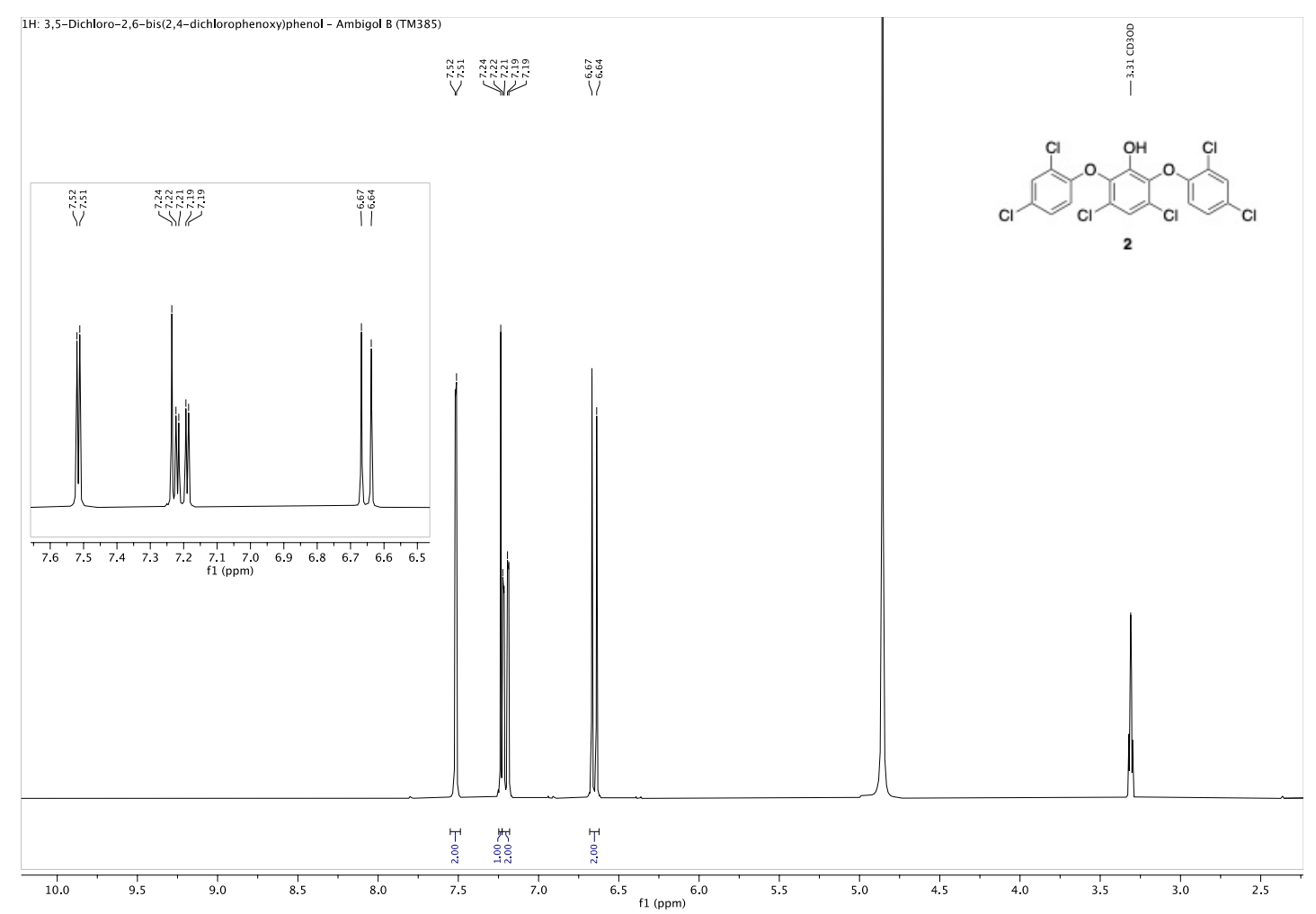

Fig. S18 ${ }^{1} \mathrm{H}-\mathrm{NMR}$ spectrum of ambigol B (2), measured in MeOD-d 4 at $300 \mathrm{MHz}$.

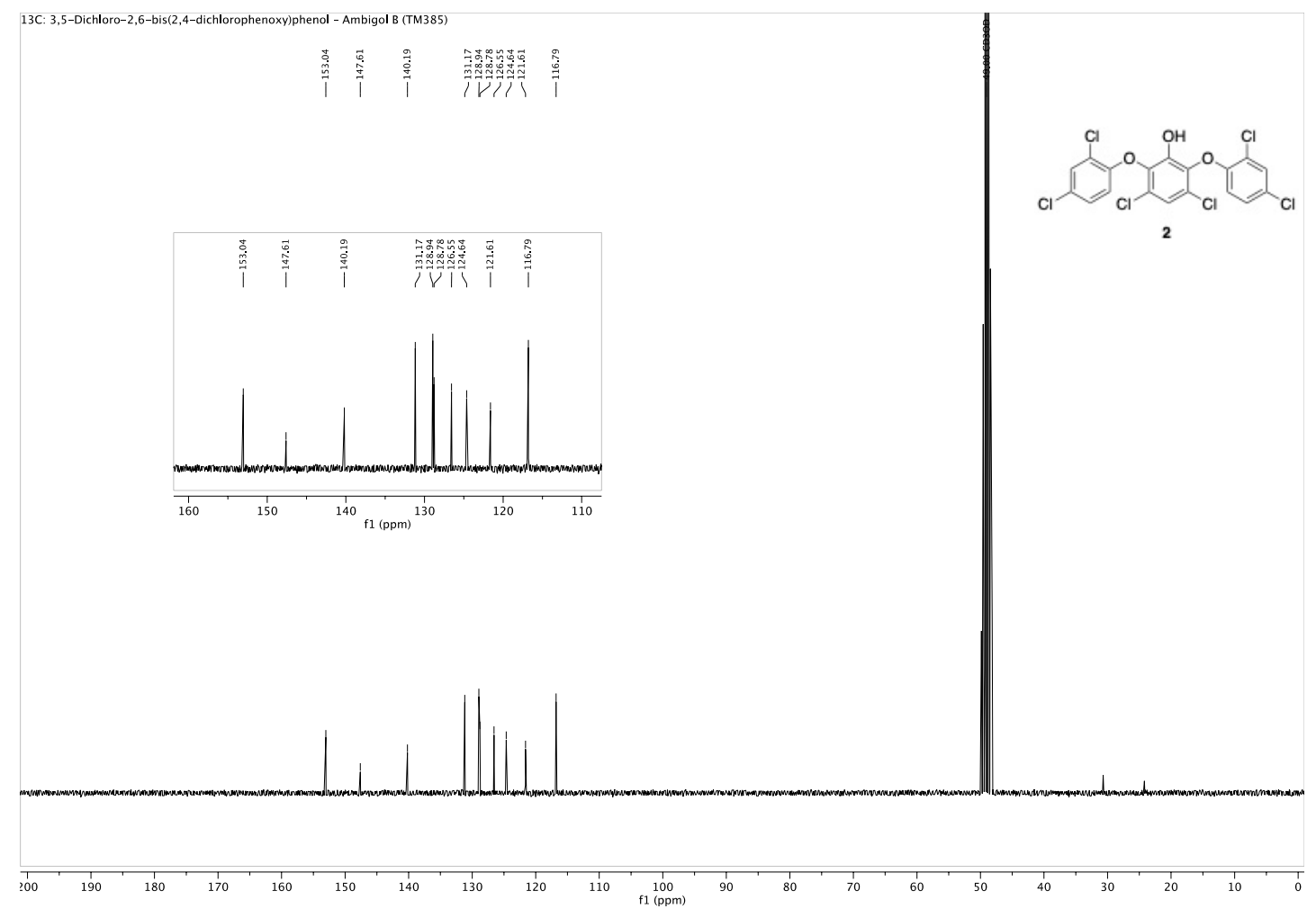

Fig. S19 ${ }^{13}$ C-NMR spectrum of ambigol B (2), measured in MeOD-d $\mathrm{d}_{4}$ at $75 \mathrm{MHz}$. 


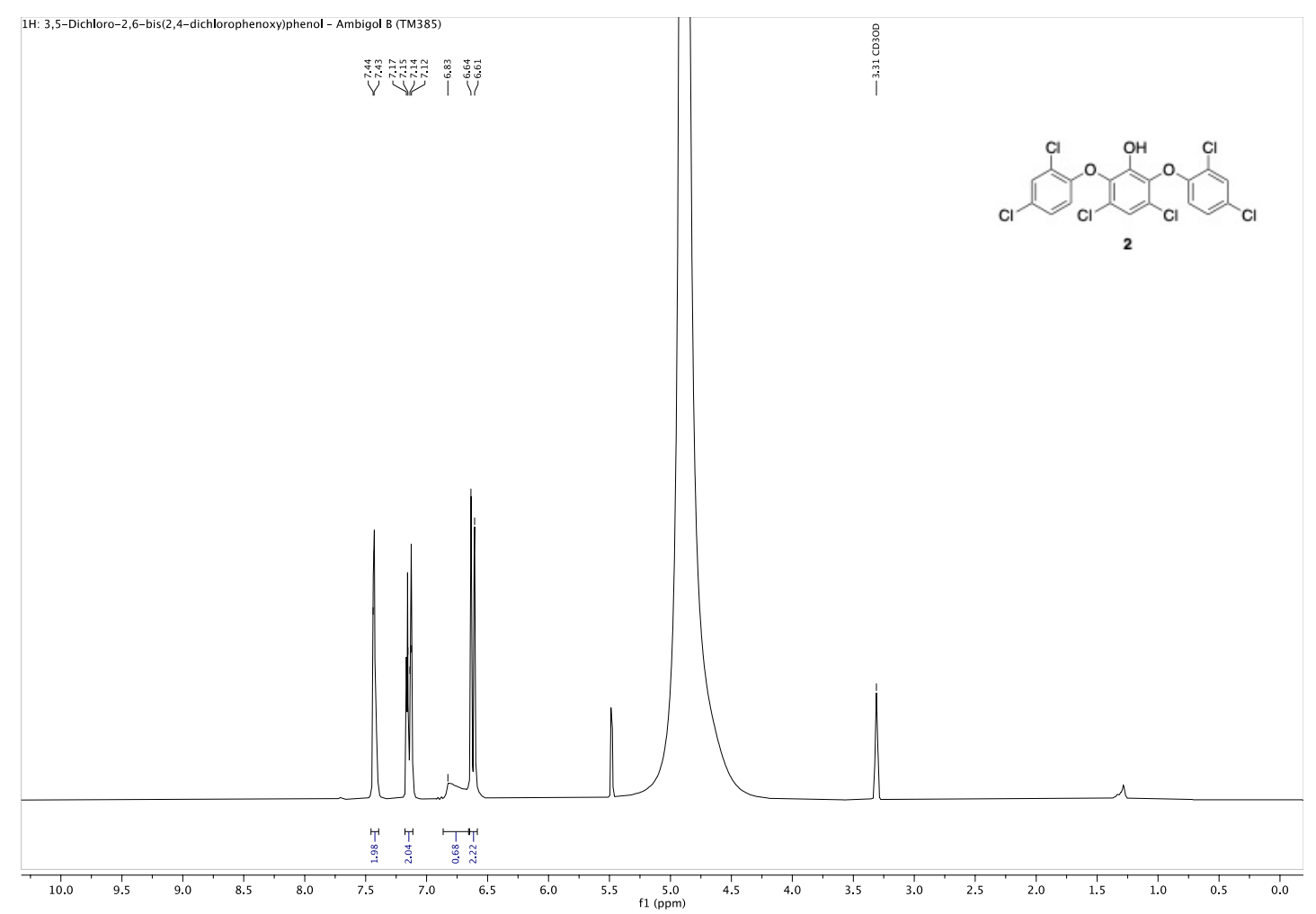

Fig. S20 ${ }^{1} \mathrm{H}-\mathrm{NMR}$ spectrum of ambigol B (2), measured in MeOD- $\mathrm{d}_{4}$ under basic conditions (one drop of $1 \mathrm{~N}$ $\mathrm{NaOH})$ at $300 \mathrm{MHz}$.

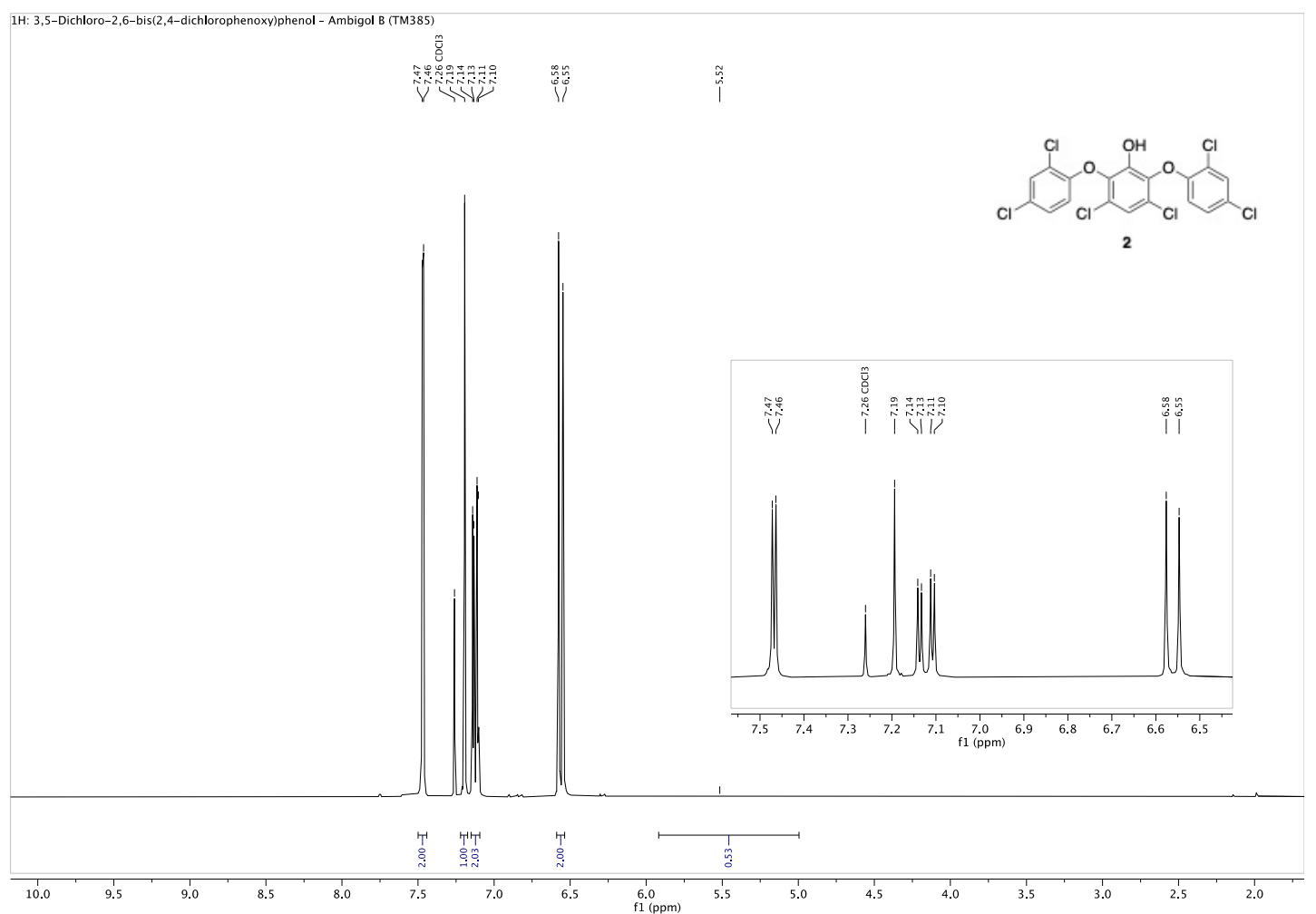

Fig. S21 ${ }^{1} \mathrm{H}-\mathrm{NMR}$ spectrum of ambigol $\mathrm{B}(2)$, measured in $\mathrm{CDCl}_{3}$ at $300 \mathrm{MHz}$. 

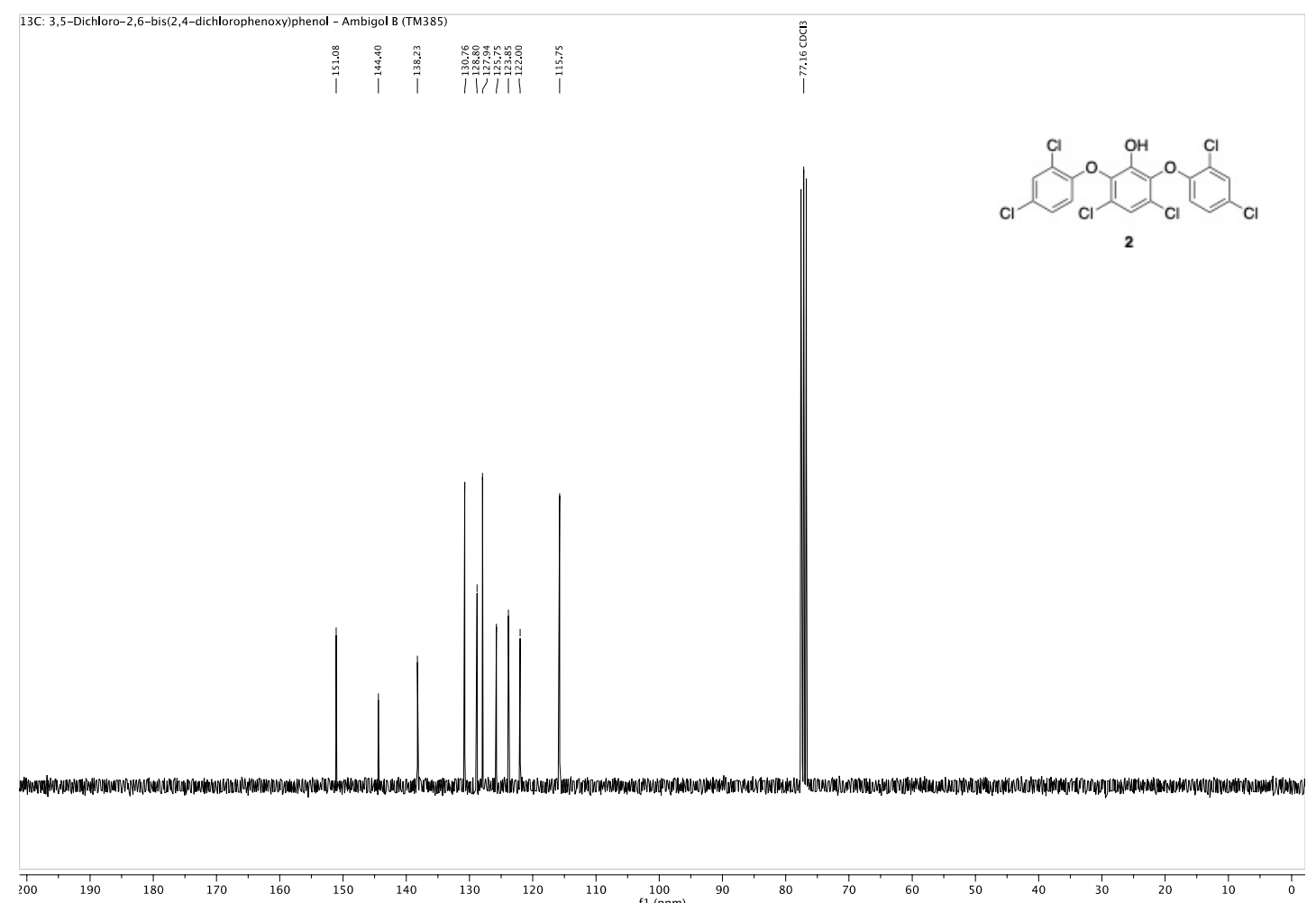

Fig. S22 ${ }^{13} \mathrm{C}$-NMR spectrum of ambigol B (2), measured in $\mathrm{CDCl}_{3}$ at $300 \mathrm{MHz}$. 


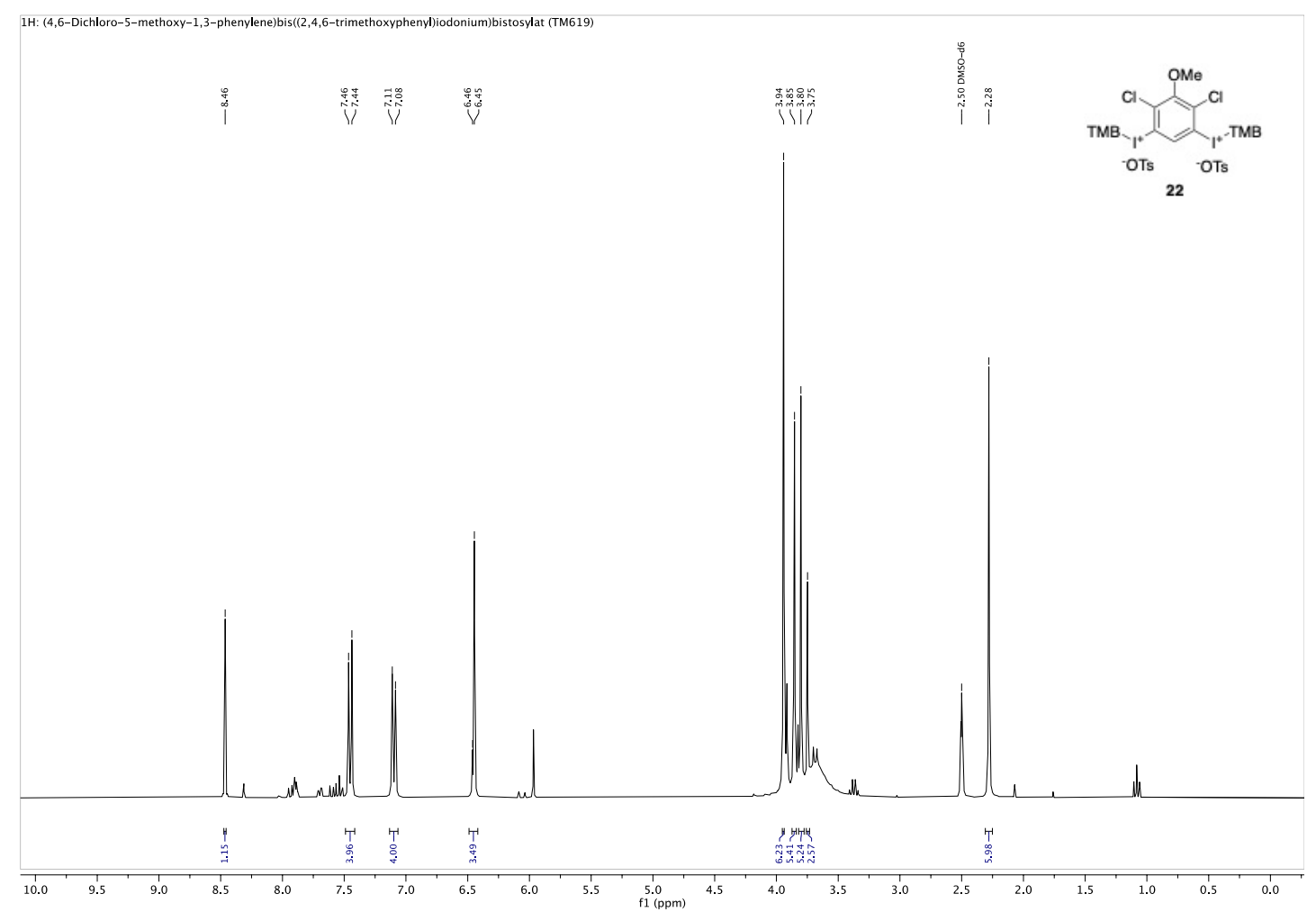

Fig. S23 ${ }^{1} \mathrm{H}$-NMR spectrum of (4,6-dichloro-5-methoxy-1,3-phenylene)bis((2,4,6-trimethoxyphenyl)iodonium) bistosylat (22), measured in DMSO-d6 at $300 \mathrm{MHz}$. 


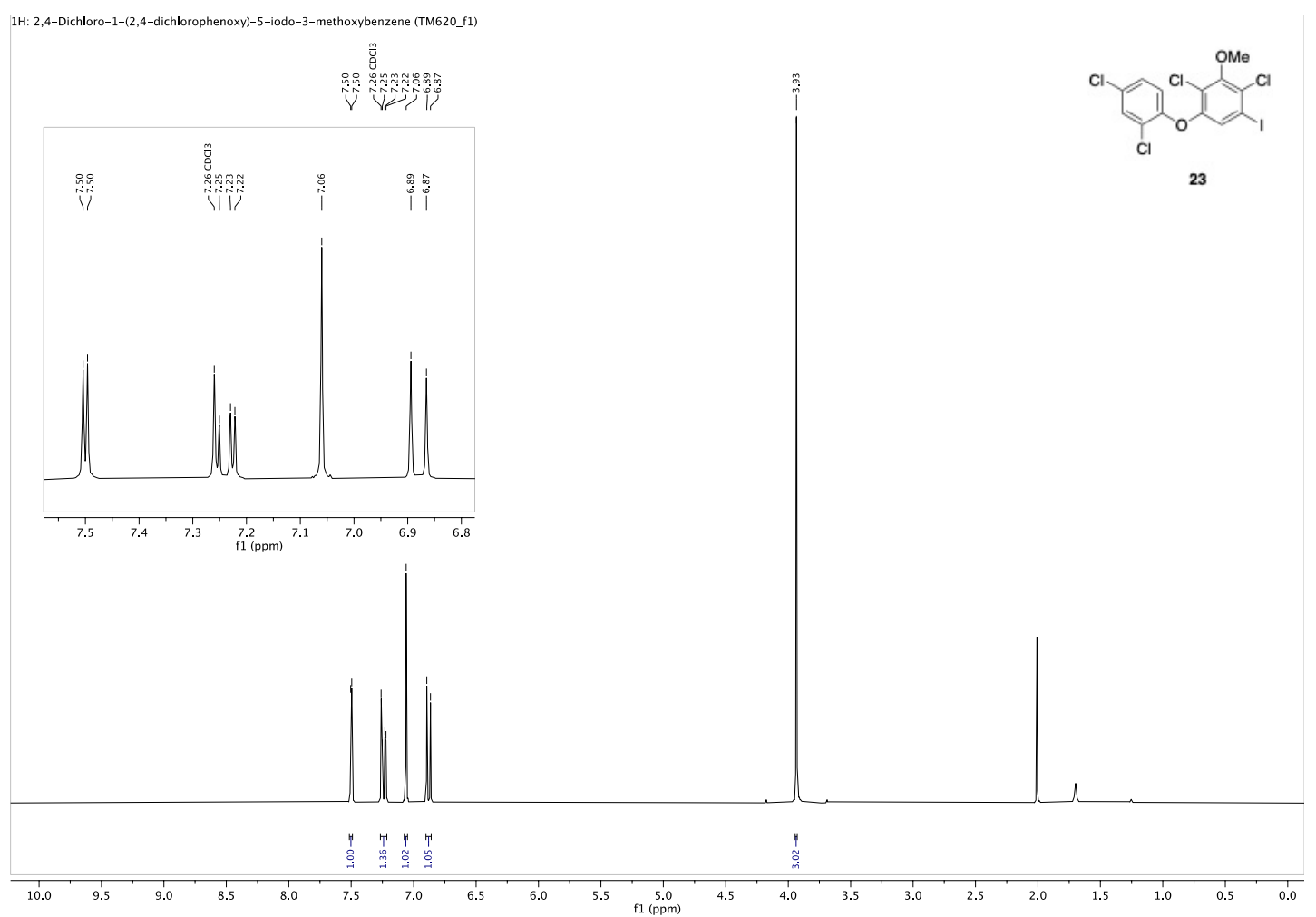

Fig. S24 ${ }^{1} \mathrm{H}-\mathrm{NMR}$ spectrum of 2,4-dichloro-1-(2,4-dichlorophenoxy)-5-iodo-3-methoxybenzene (23), measured in $\mathrm{CDCl}_{3}$ at $300 \mathrm{MHz}$.

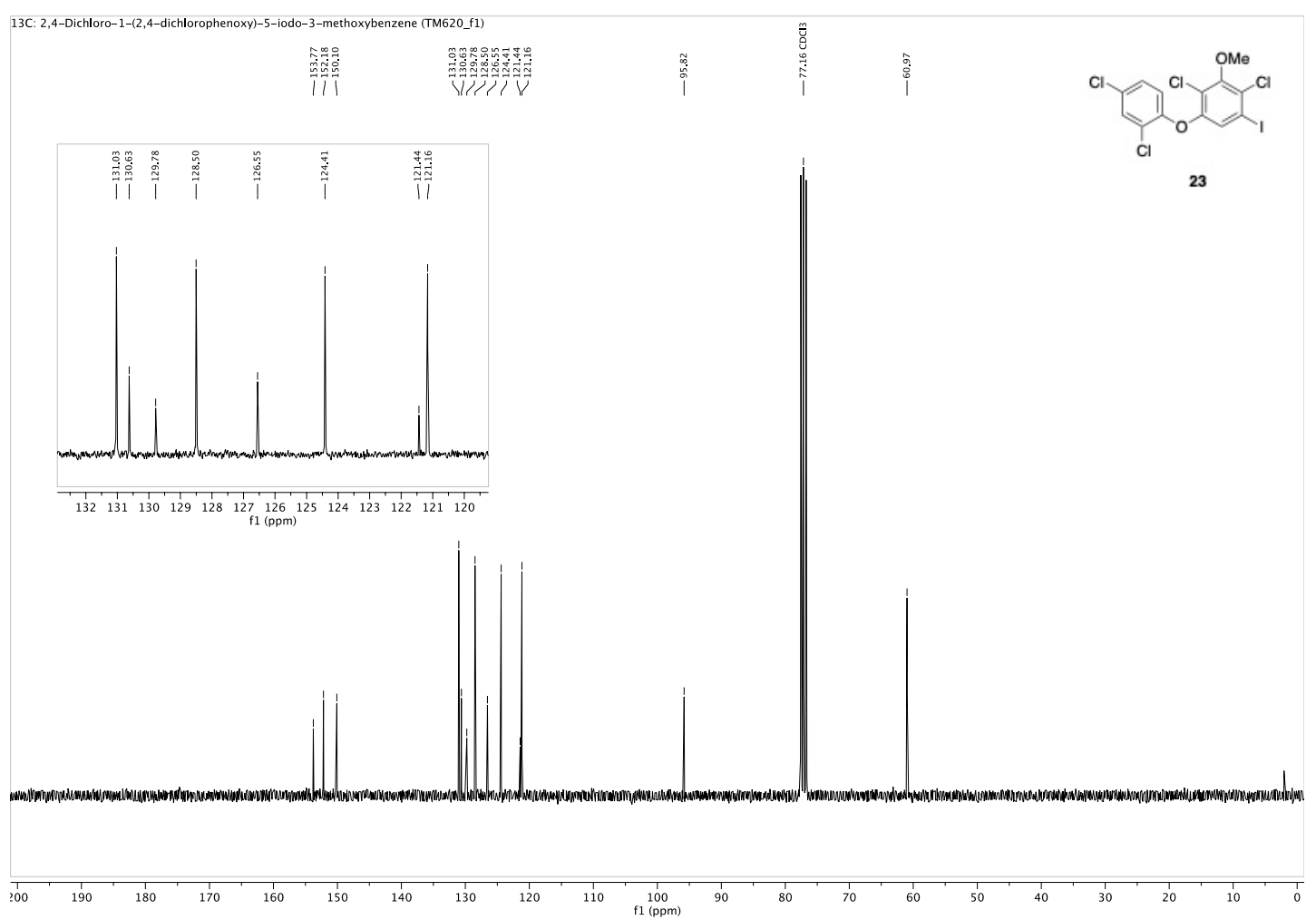

Fig. S25 ${ }^{13} \mathrm{C}$-NMR spectrum of 2,4-dichloro-1-(2,4-dichlorophenoxy)-5-iodo-3-methoxybenzene (23), measured in $\mathrm{CDCl}_{3}$ at $75 \mathrm{MHz}$. 


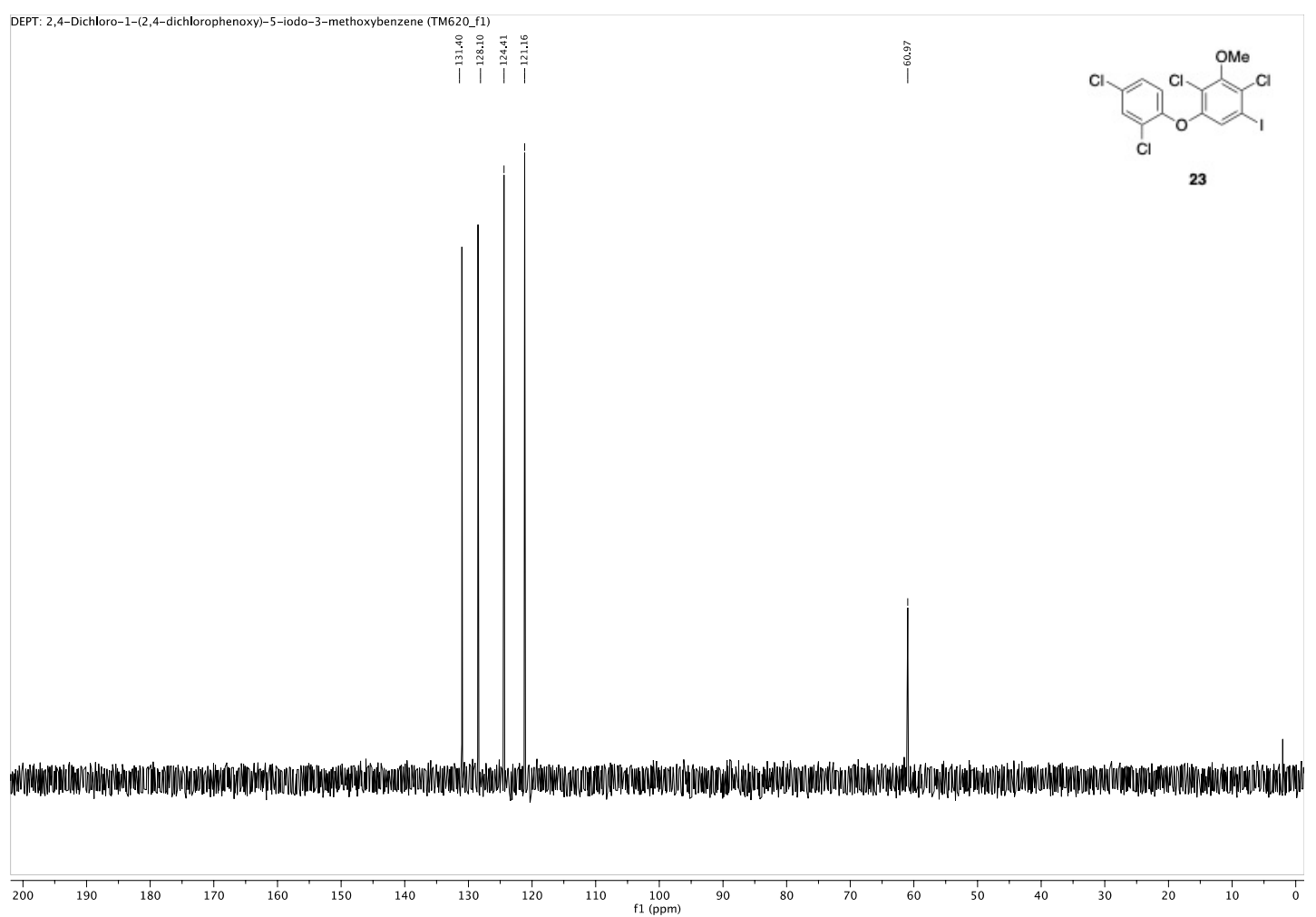

Fig. S26 DEPT spectrum of 2,4-dichloro-1-(2,4-dichlorophenoxy)-5-iodo-3-methoxybenzene (23), measured in $\mathrm{CDCl}_{3}$ at $75 \mathrm{MHz}$. 


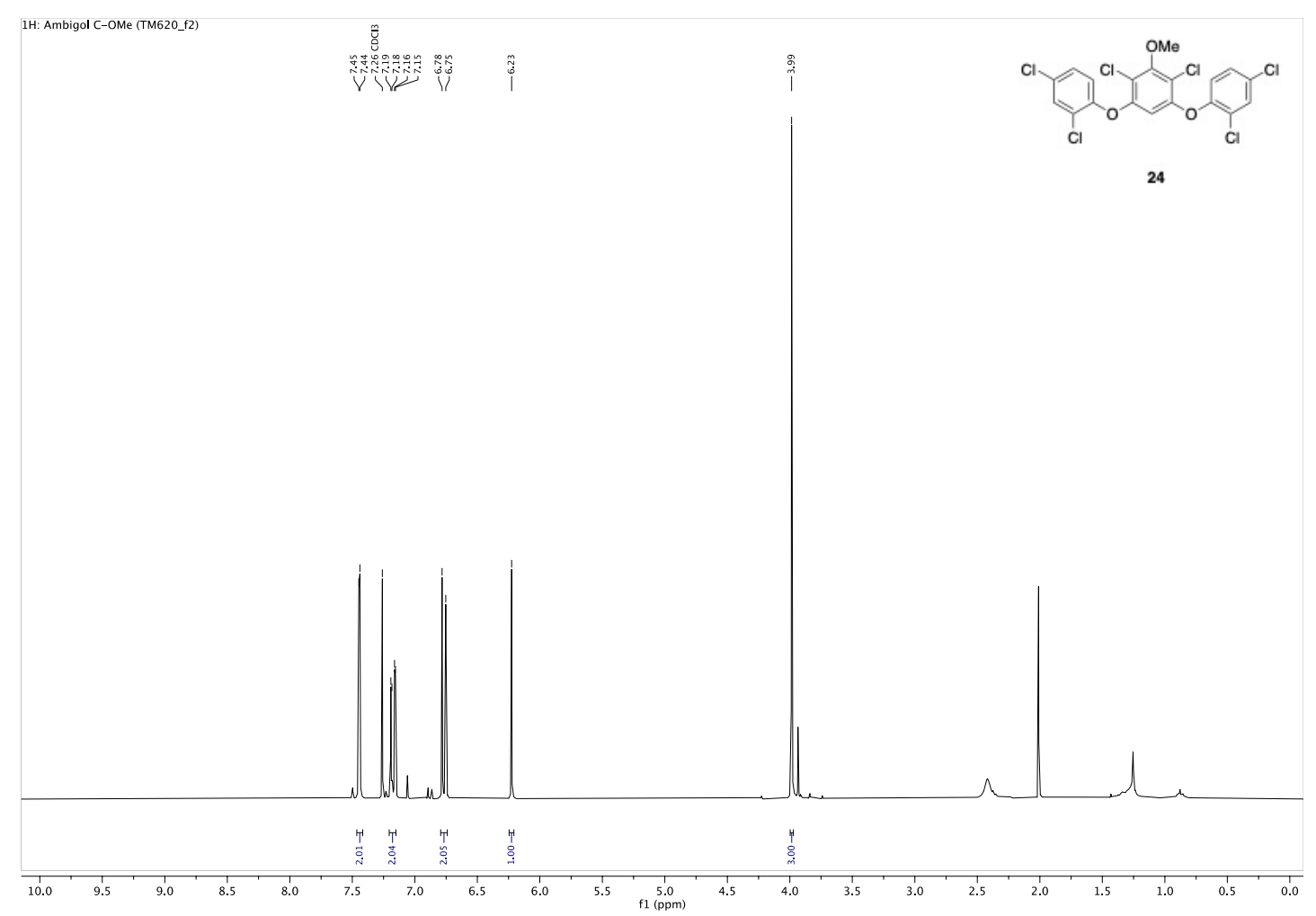

Fig. S27 ${ }^{1}$ H-NMR spectrum of 4,4'-((4,6-dichloro-5-methoxy-1,3-phenylene)bis(oxy))bis(1,3-dichlorobenzene) (24), measured in $\mathrm{CDCl}_{3}$ at $300 \mathrm{MHz}$.

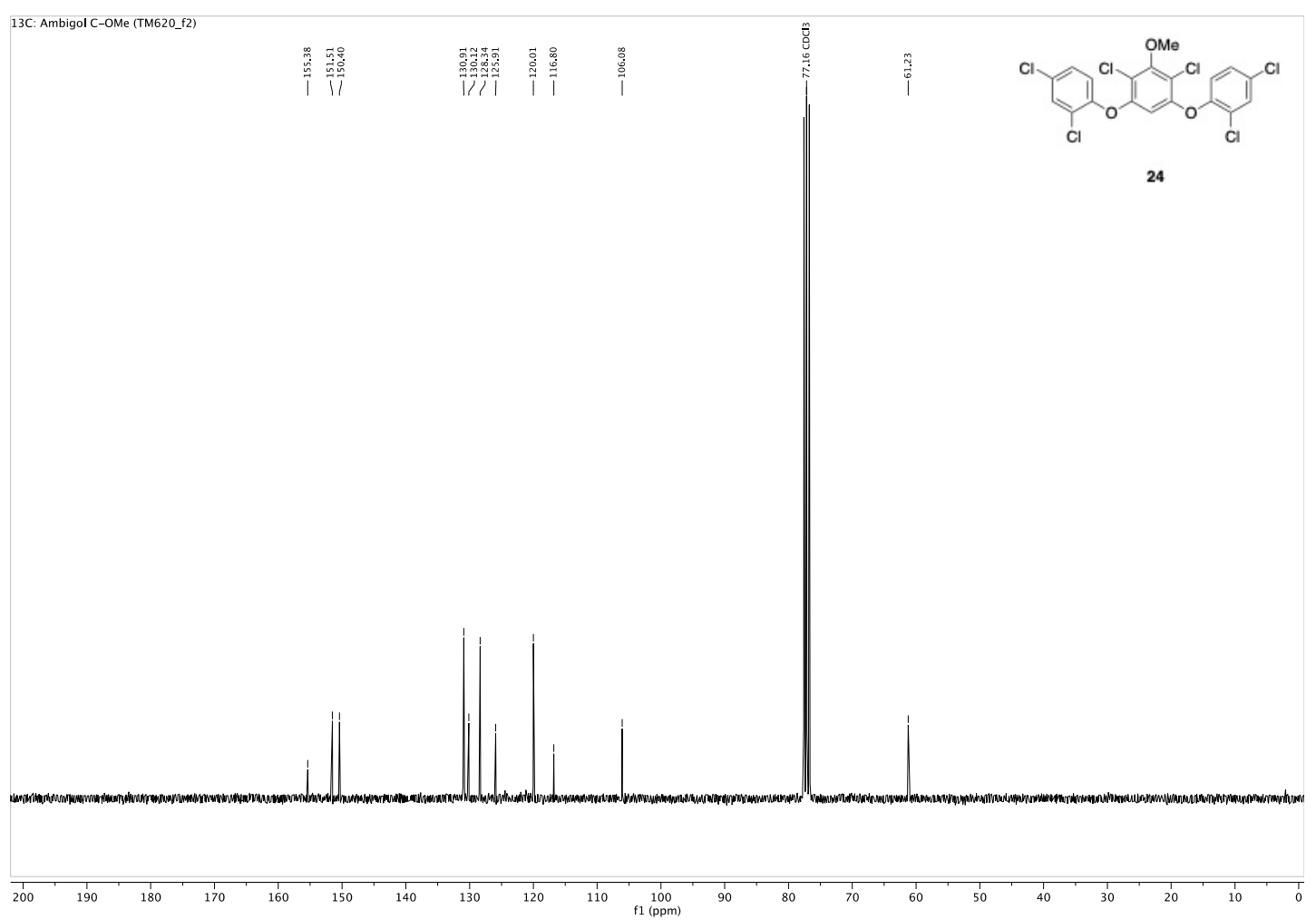

Fig. S28 ${ }^{13}$ C-NMR spectrum of 4,4'-((4,6-dichloro-5-methoxy-1,3-phenylene)bis(oxy))bis(1,3-dichlorobenzene) (24), measured in $\mathrm{CDCl}_{3}$ at $75 \mathrm{MHz}$. 


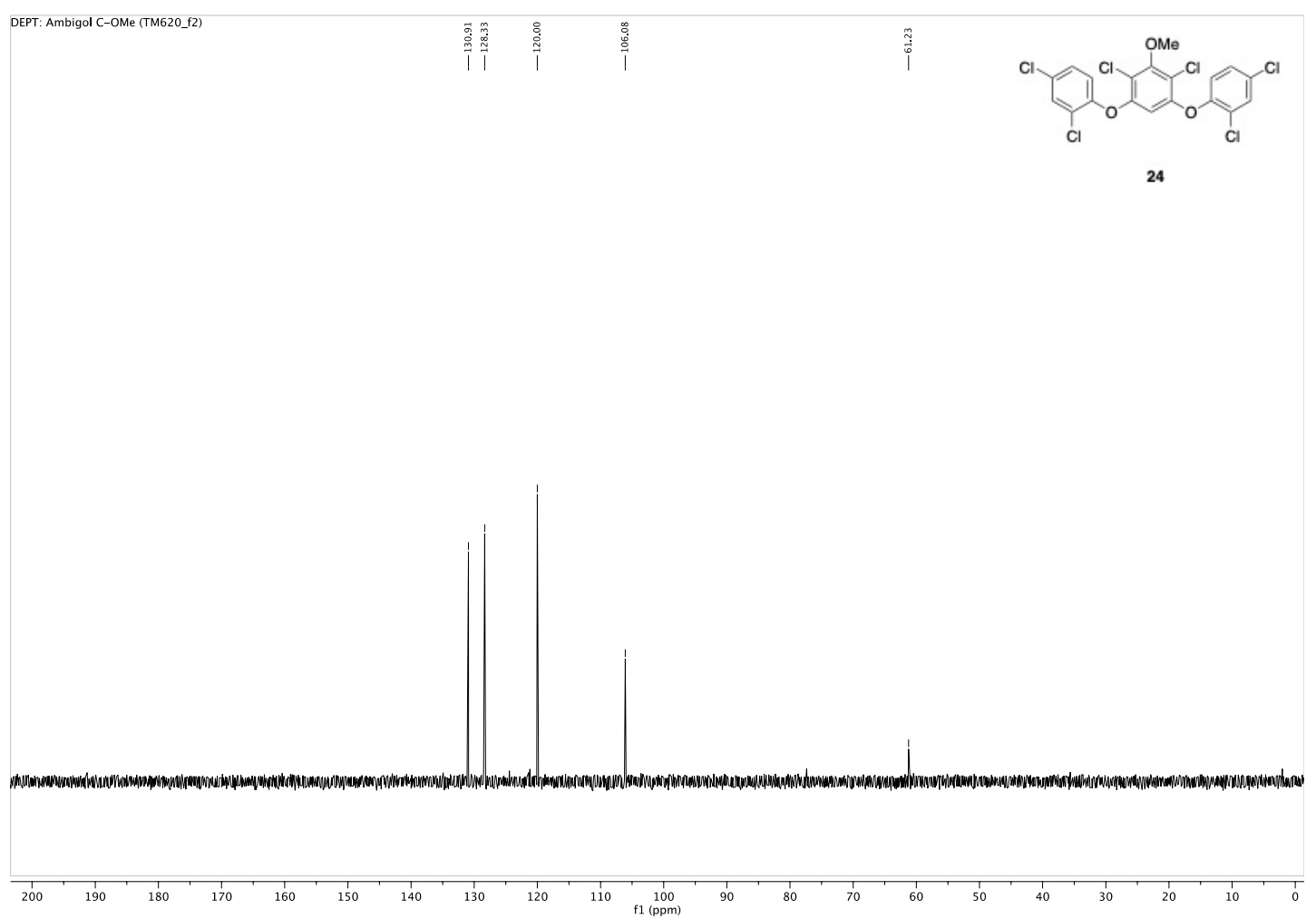

Fig. S29 DEPT spectrum of 4,4'-((4,6-dichloro-5-methoxy-1,3-phenylene)bis(oxy))bis(1,3-dichlorobenzene) (24), measured in $\mathrm{CDCl}_{3}$ at $75 \mathrm{MHz}$. 


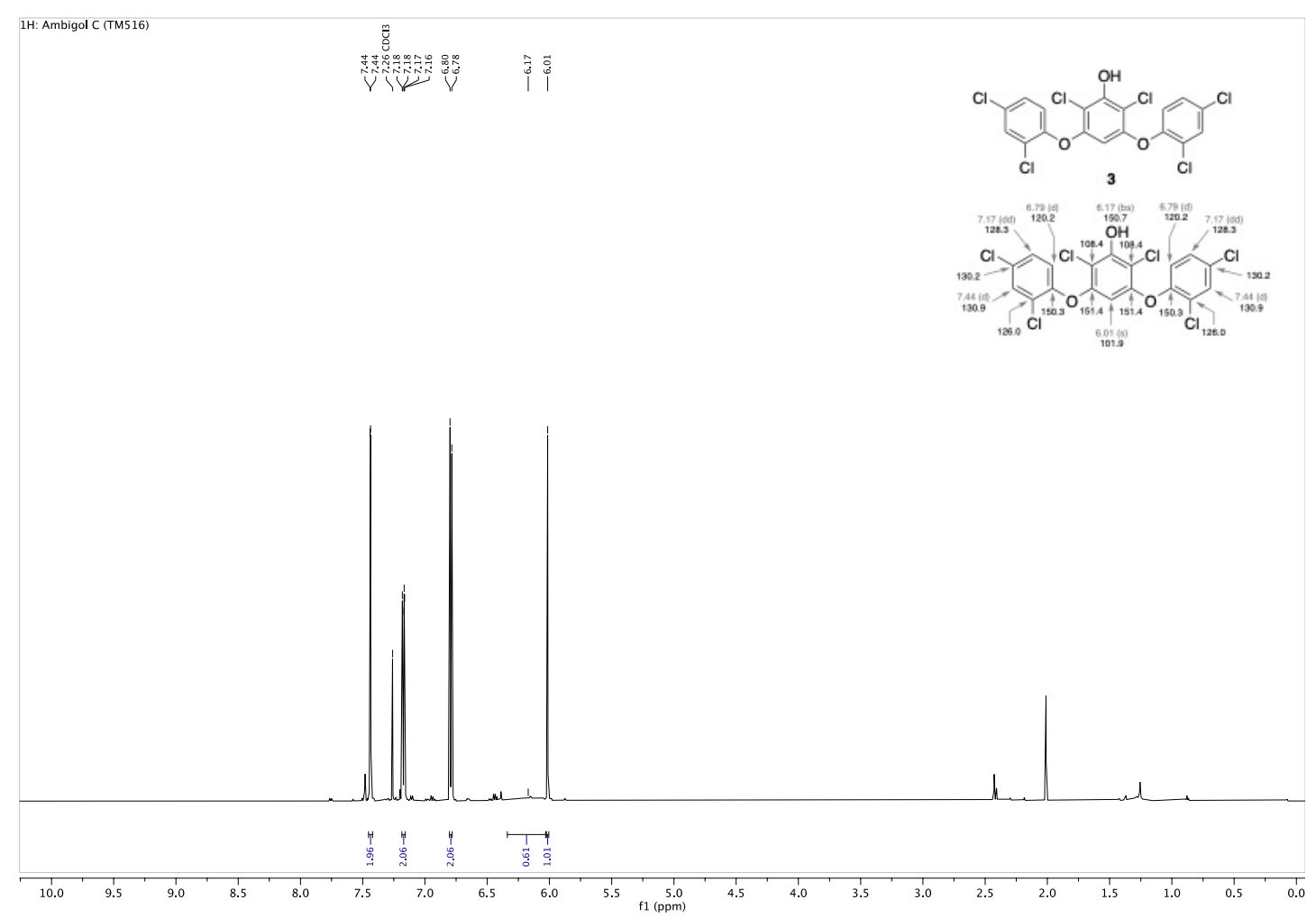

Fig. S30 ${ }^{1} \mathrm{H}$-NMR spectrum of ambigol C (3), measured in $\mathrm{CDCl}_{3}$ at $300 \mathrm{MHz}$.

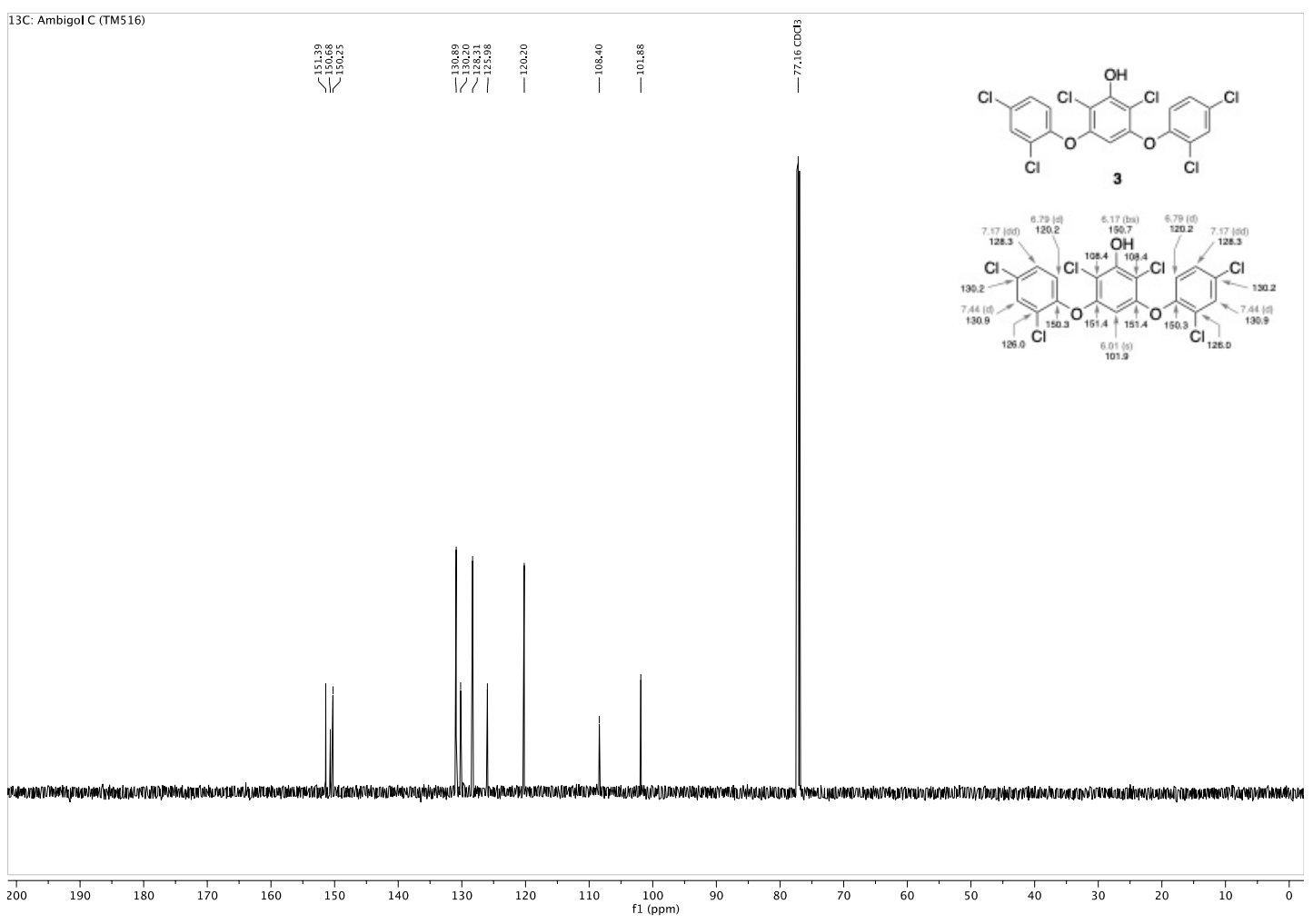

Fig. S31 ${ }^{13} \mathrm{C}-\mathrm{NMR}$ spectrum of ambigol C (3), measured in $\mathrm{CDCl}_{3}$ at $75 \mathrm{MHz}$. 

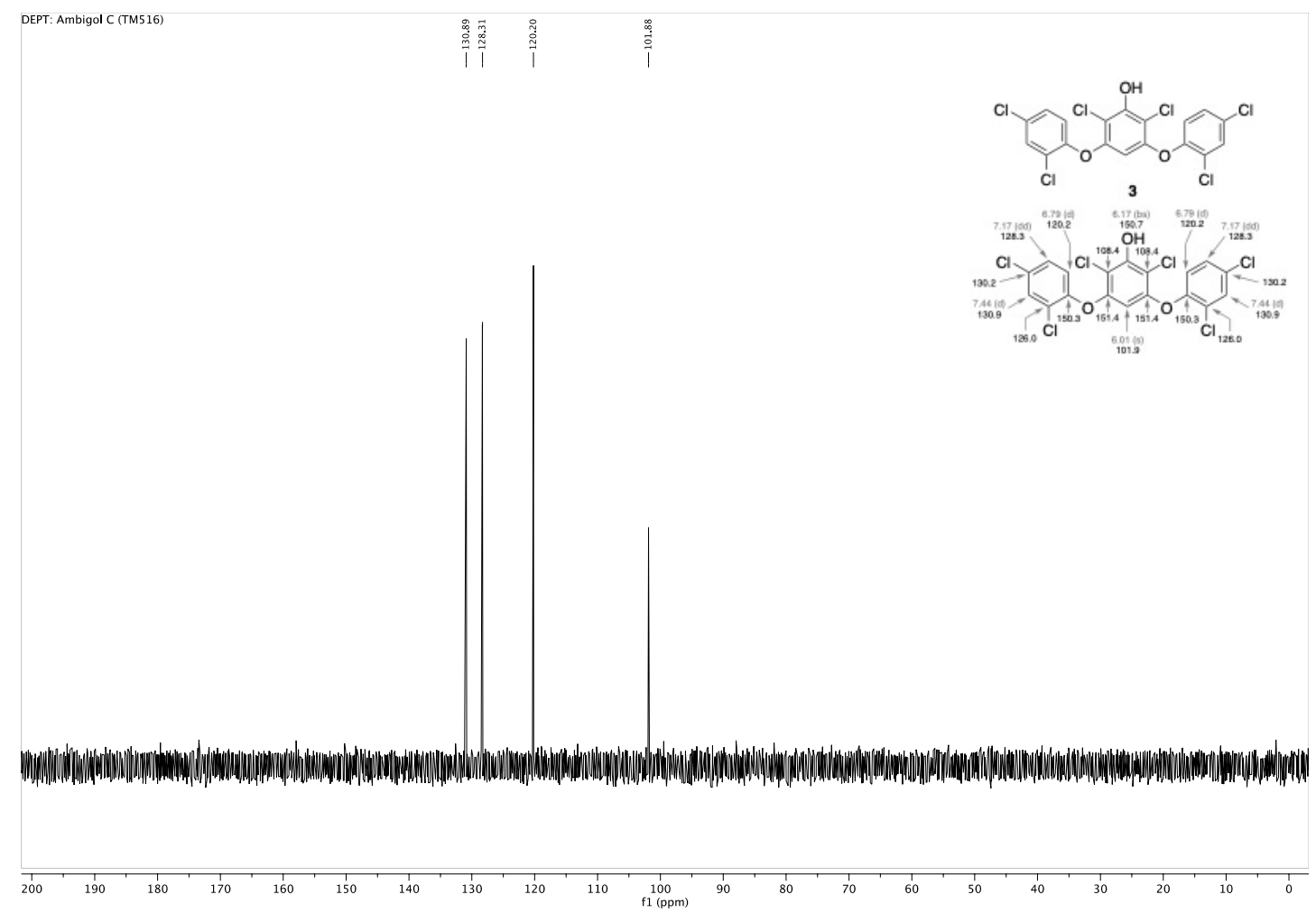

Fig. $\mathbf{S 3 2}$ DEPT spectrum of ambigol C (3), measured in $\mathrm{CDCl}_{3}$ at $75 \mathrm{MHz}$.

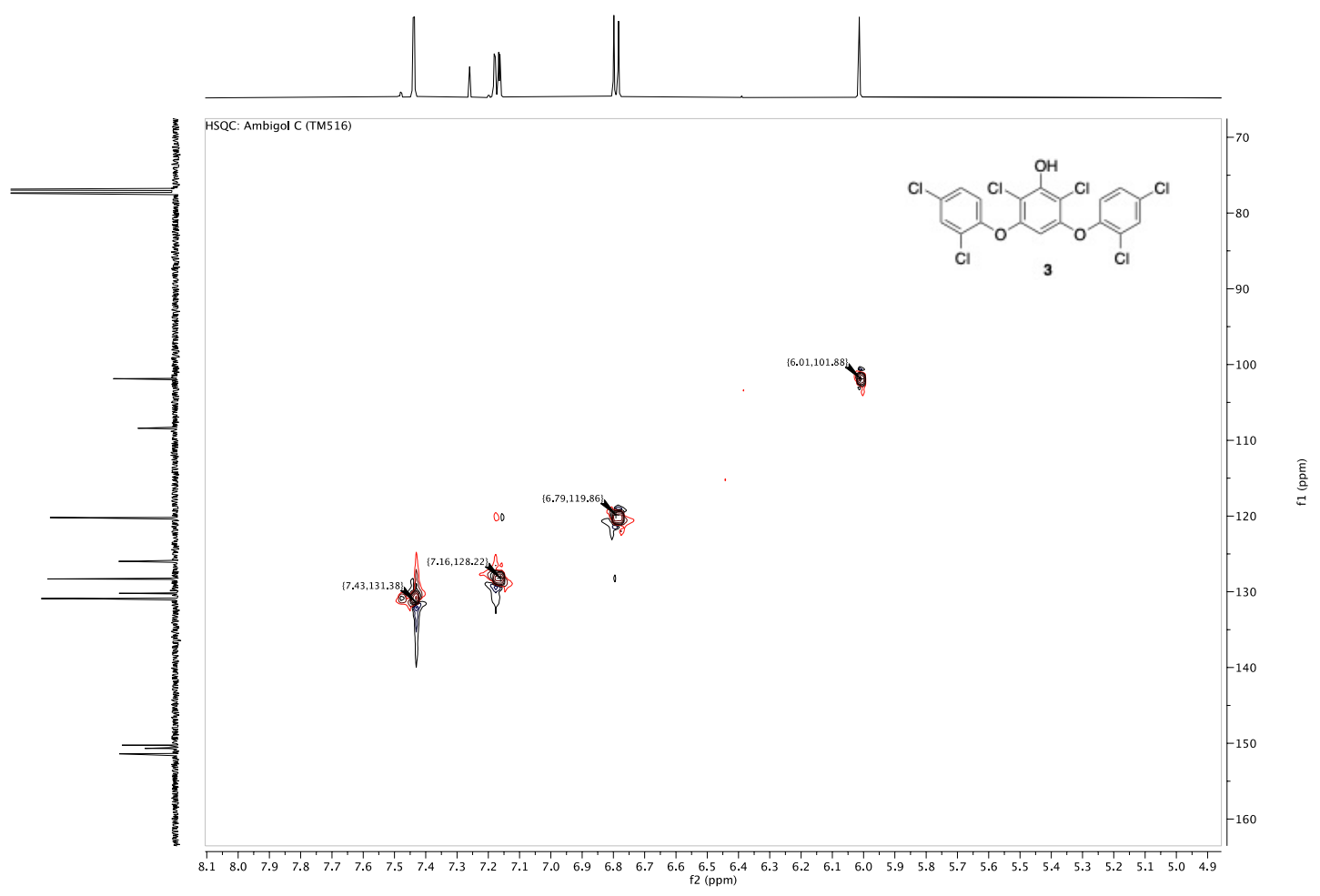

Fig. S33 HSQC spectrum of ambigol C (3), measured in $\mathrm{CDCl}_{3}$. 


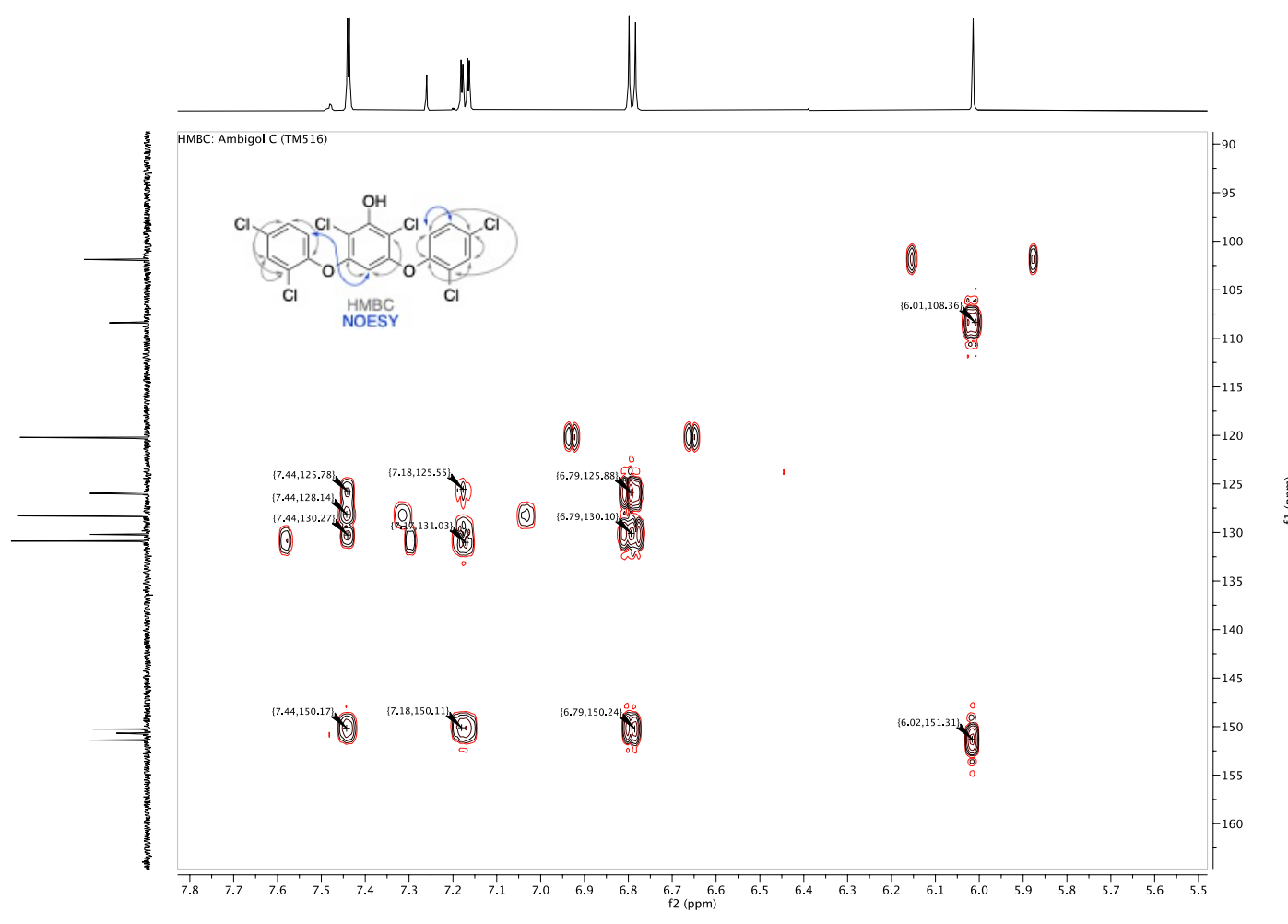

Fig. S34 $\mathrm{HMBC}$ spectrum of ambigol C (3), measured in $\mathrm{CDCl}_{3}$.

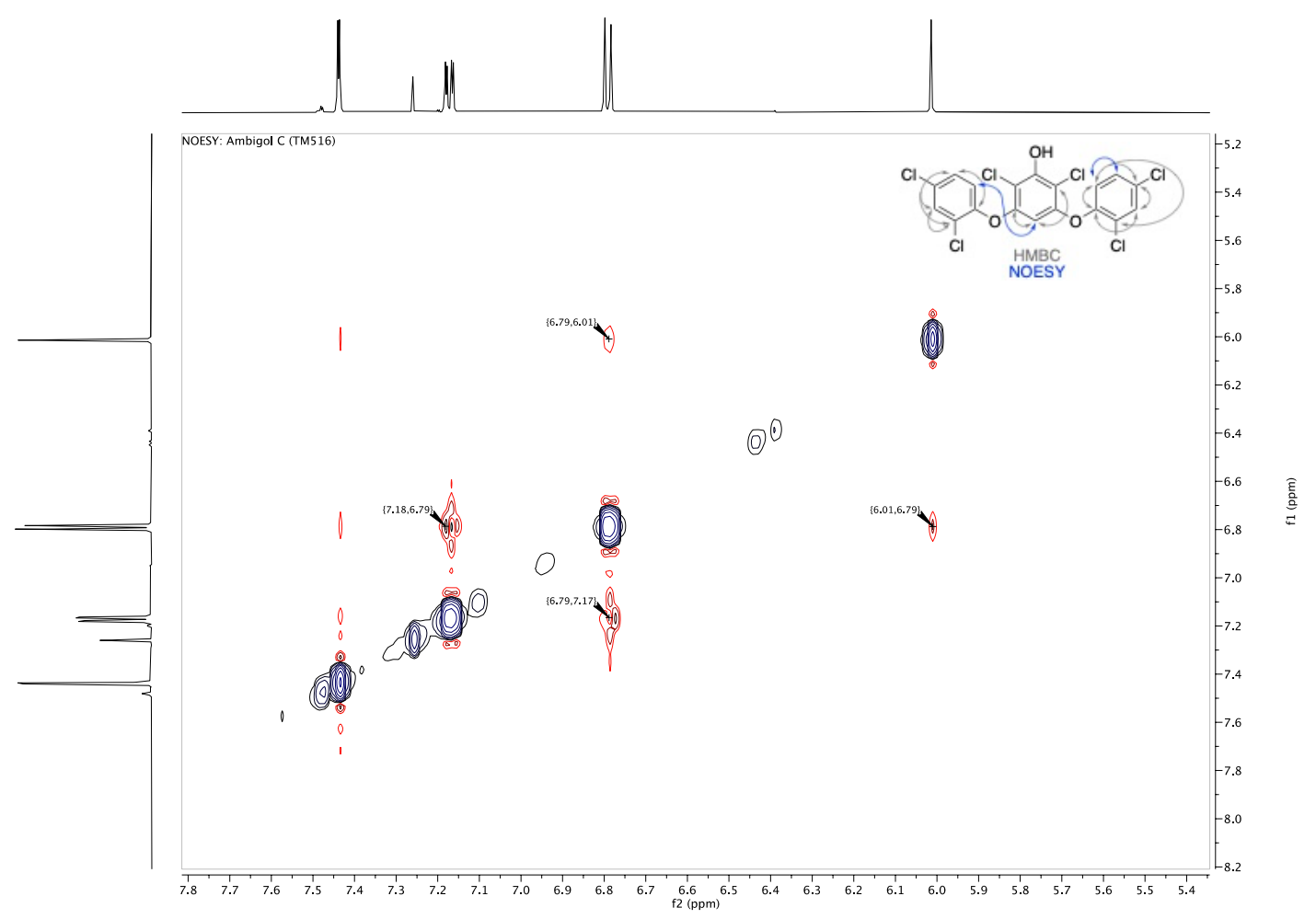

Fig. S35 NOESY spectrum of ambigol C (3), measured in $\mathrm{CDCl}_{3}$. 


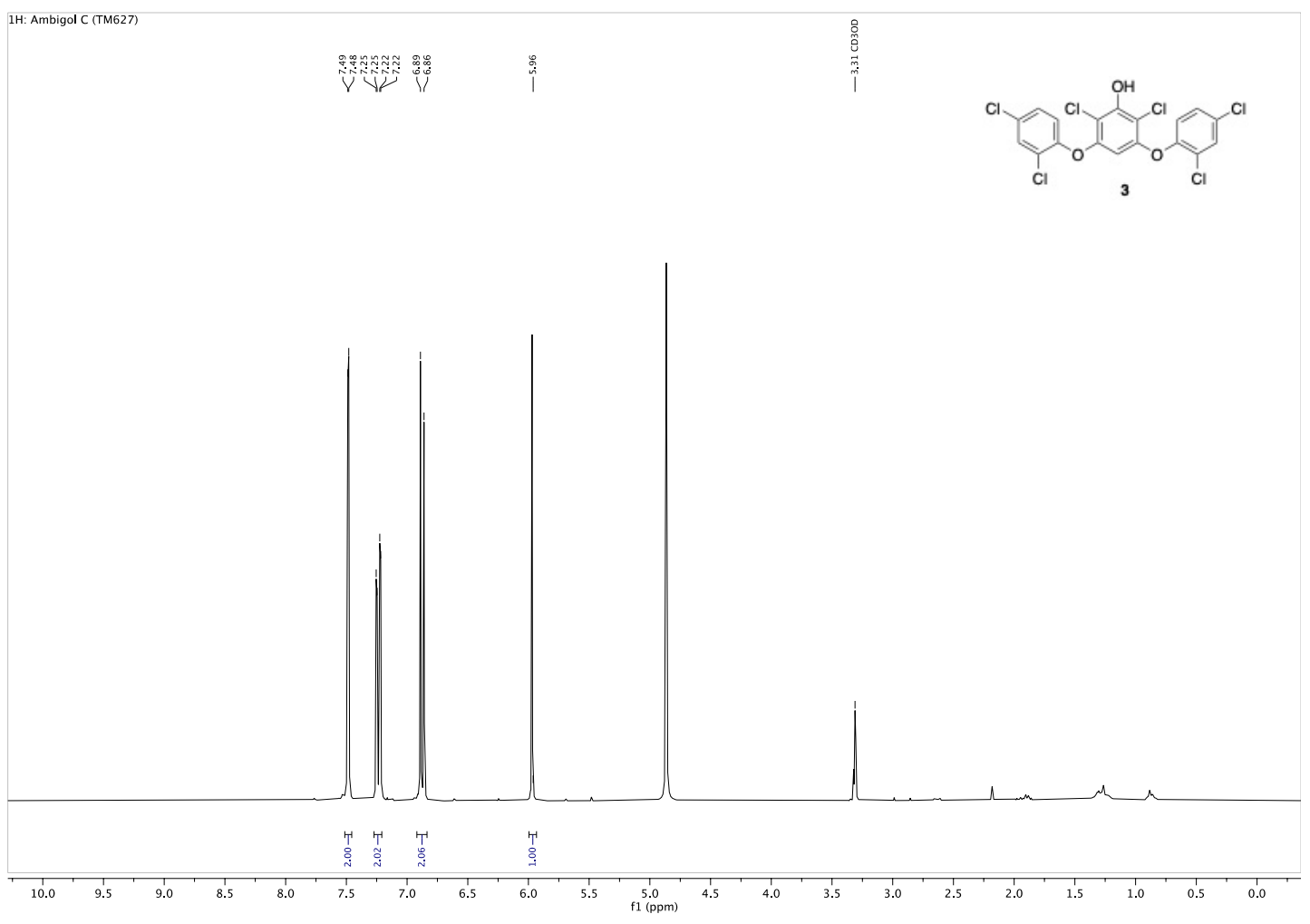

Fig. S36 ${ }^{1} \mathrm{H}-\mathrm{NMR}$ spectrum of ambigol C (3), measured in MeOD-d 4 at $300 \mathrm{MHz}$.

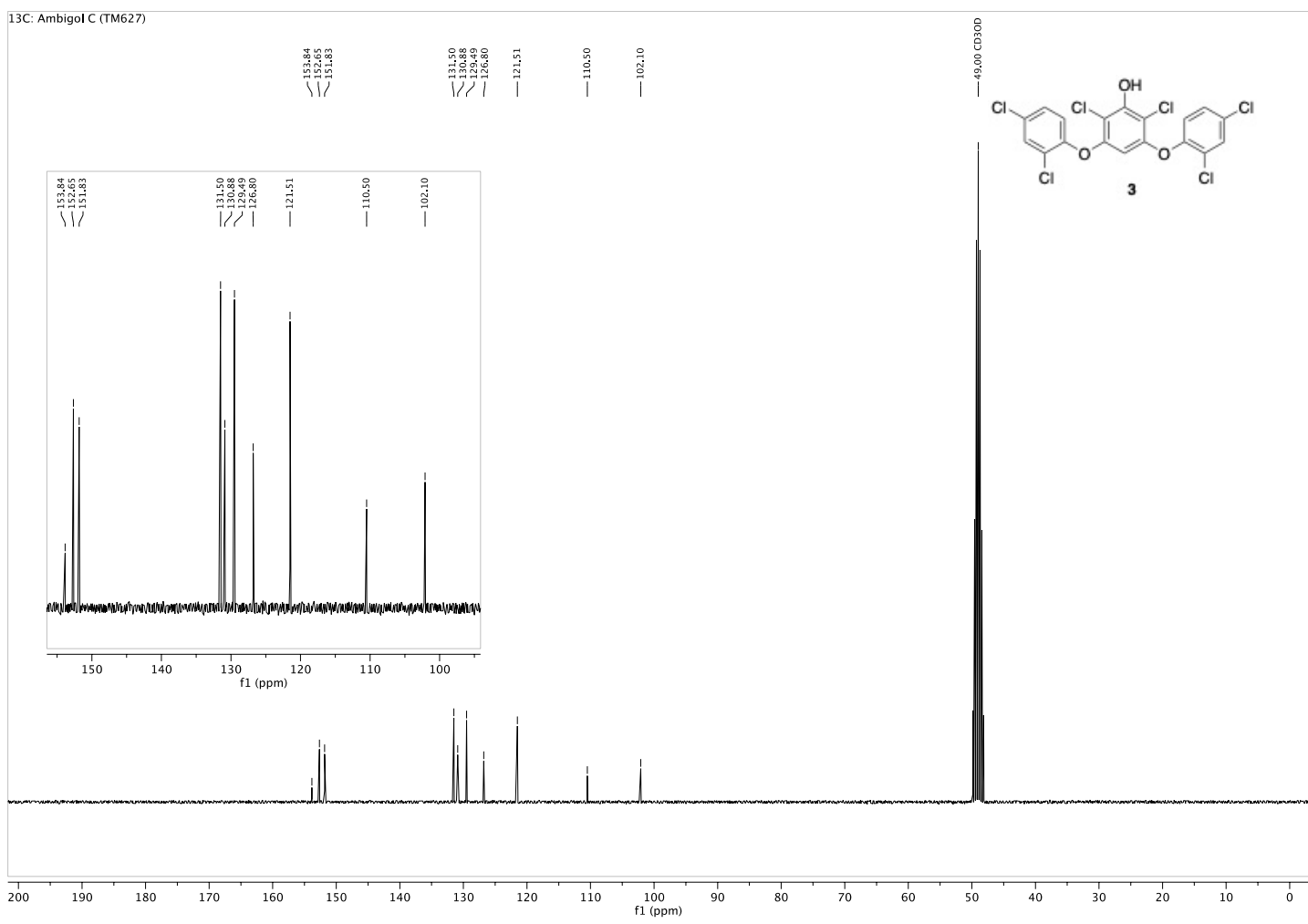

Fig. S37 ${ }^{13} \mathrm{C}-\mathrm{NMR}$ spectrum of ambigol C (3), measured in MeOD-d 4 at $75 \mathrm{MHz}$. 

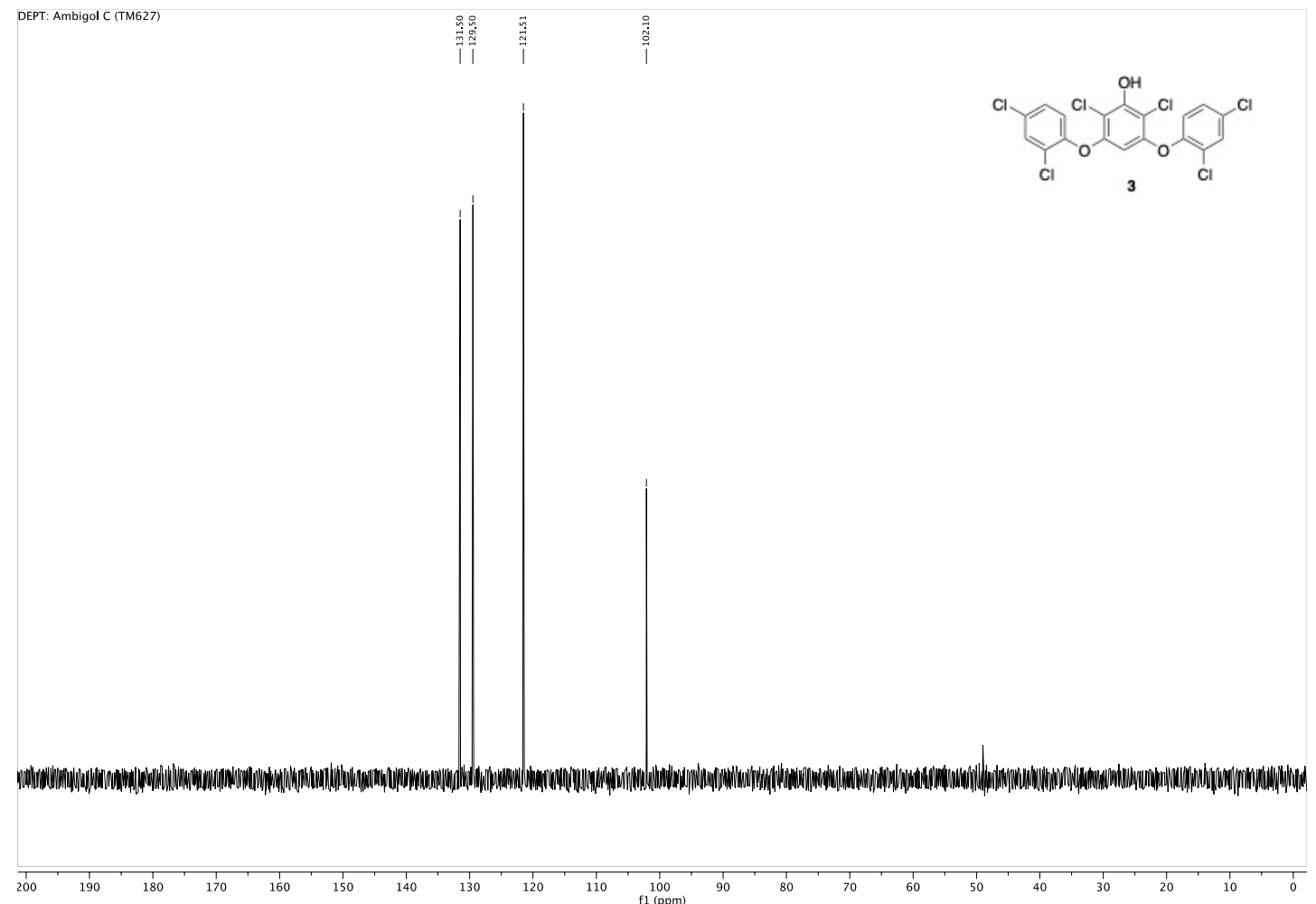

Fig. S38 DEPT spectrum of ambigol C (3), measured in MeOD-d 4 at $75 \mathrm{MHz}$. 


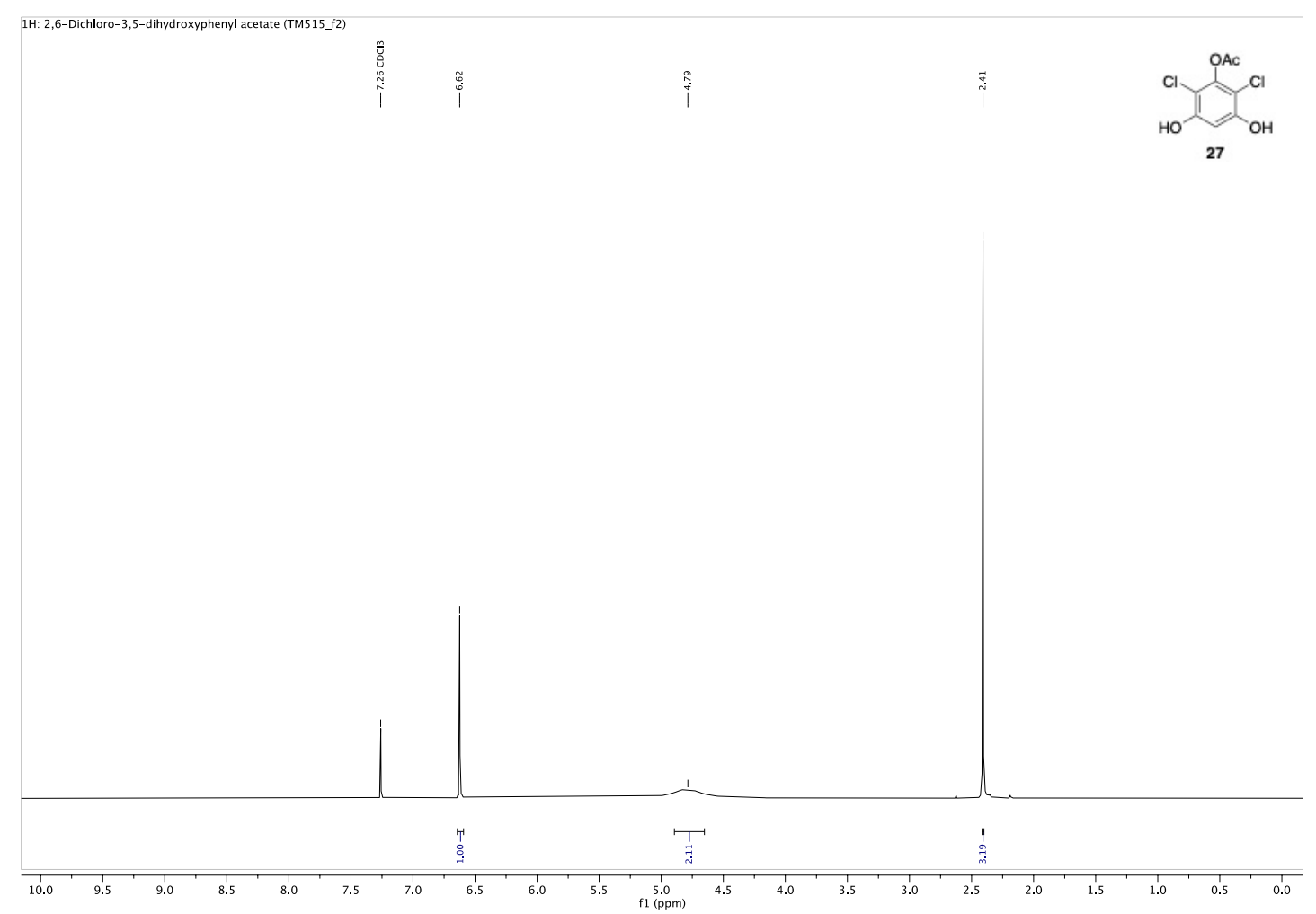

Fig. S39 ${ }^{1} \mathrm{H}$-NMR spectrum of 2,6-dichloro-3,5-dihydroxyphenyl acetate (27), measured in $\mathrm{CDCl}_{3}$ at $300 \mathrm{MHz}$.

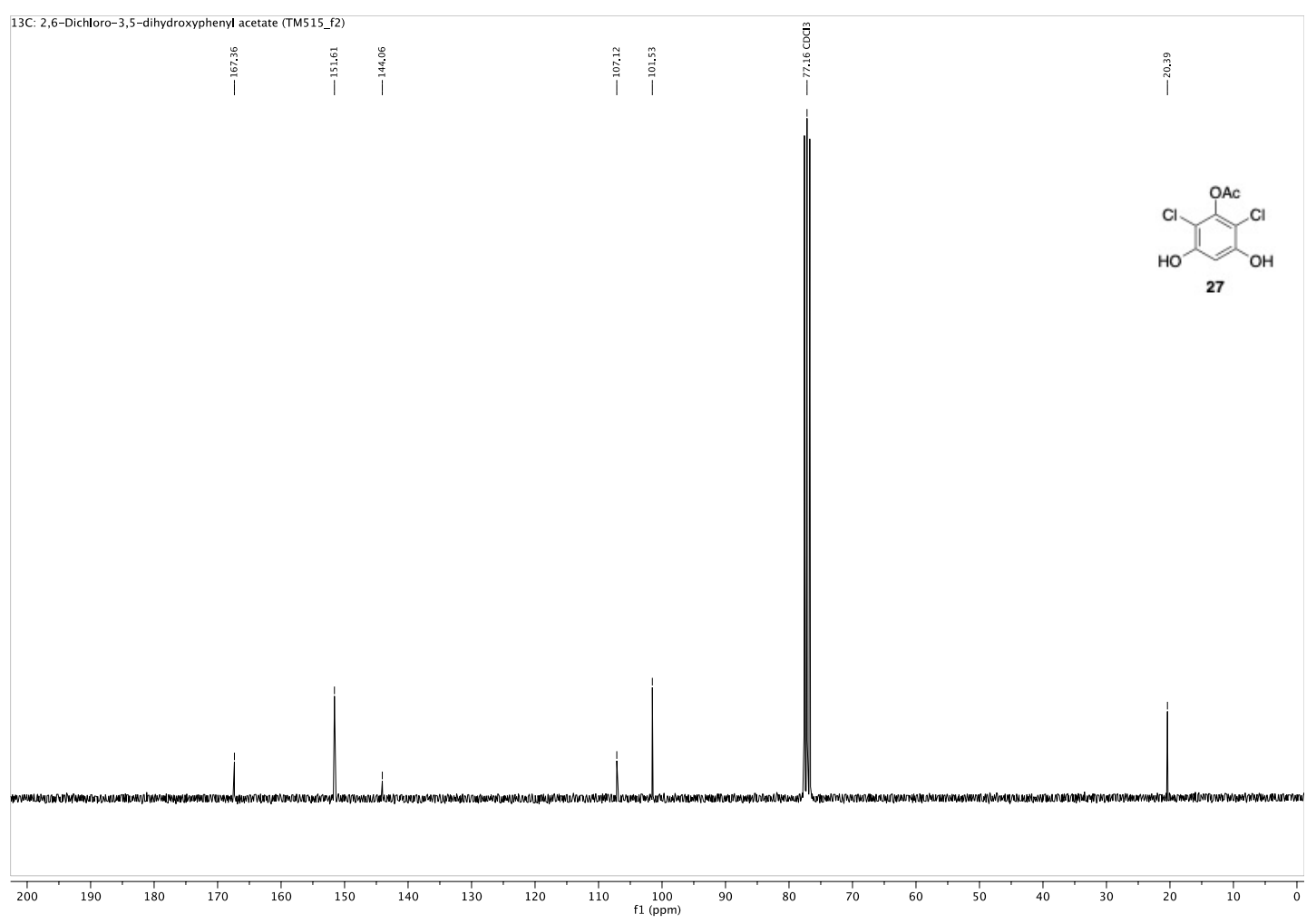

Fig. $\mathbf{S 4 0}{ }^{13} \mathrm{C}$-NMR spectrum of 2,6-dichloro-3,5-dihydroxyphenyl acetate (27), measured in $\mathrm{CDCl}_{3}$ at $75 \mathrm{MHz}$. 


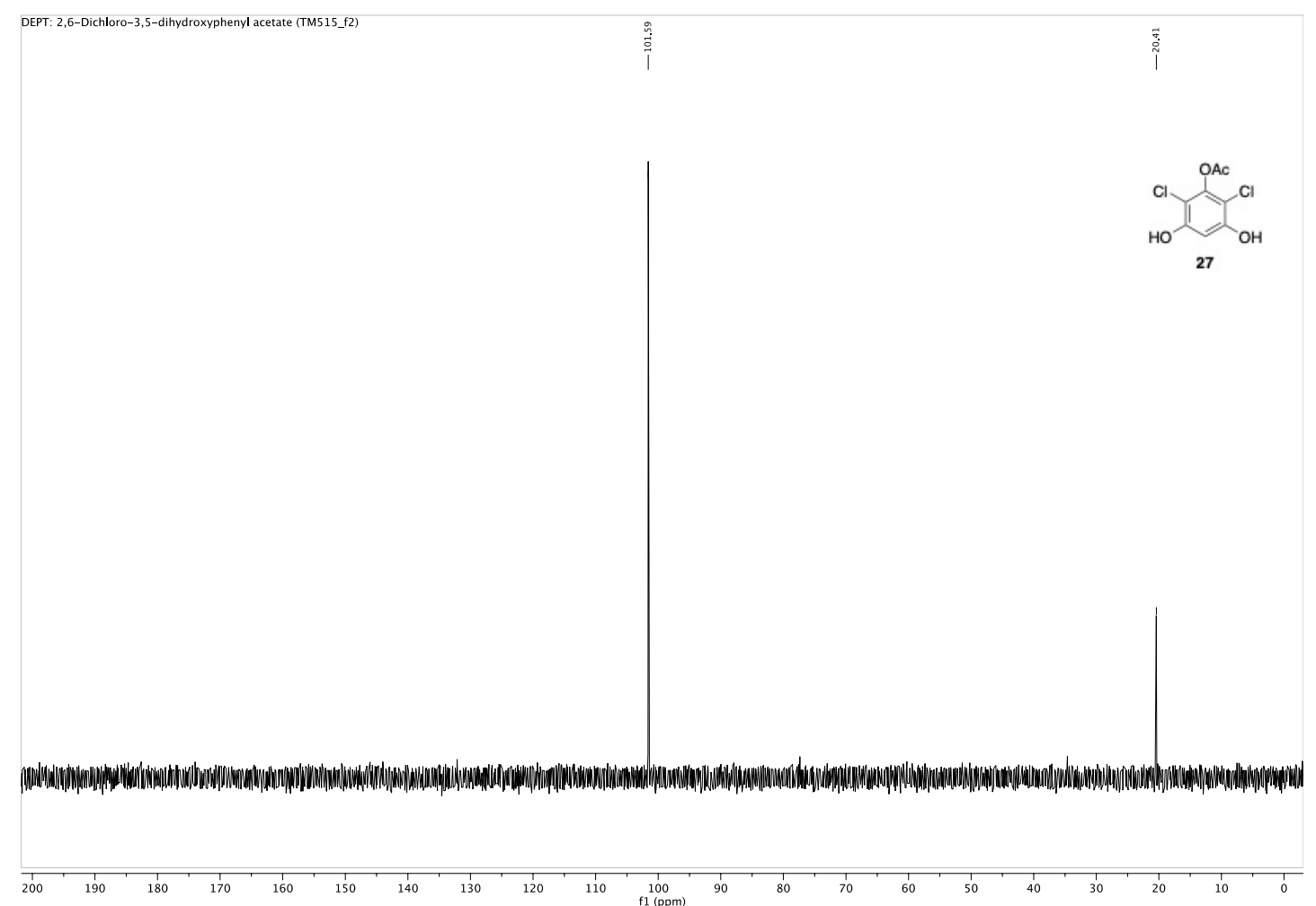

Fig. S41 DEPT spectrum of 2,6-dichloro-3,5-dihydroxyphenyl acetate (27), measured in $\mathrm{CDCl}_{3}$ at $75 \mathrm{MHz}$. 


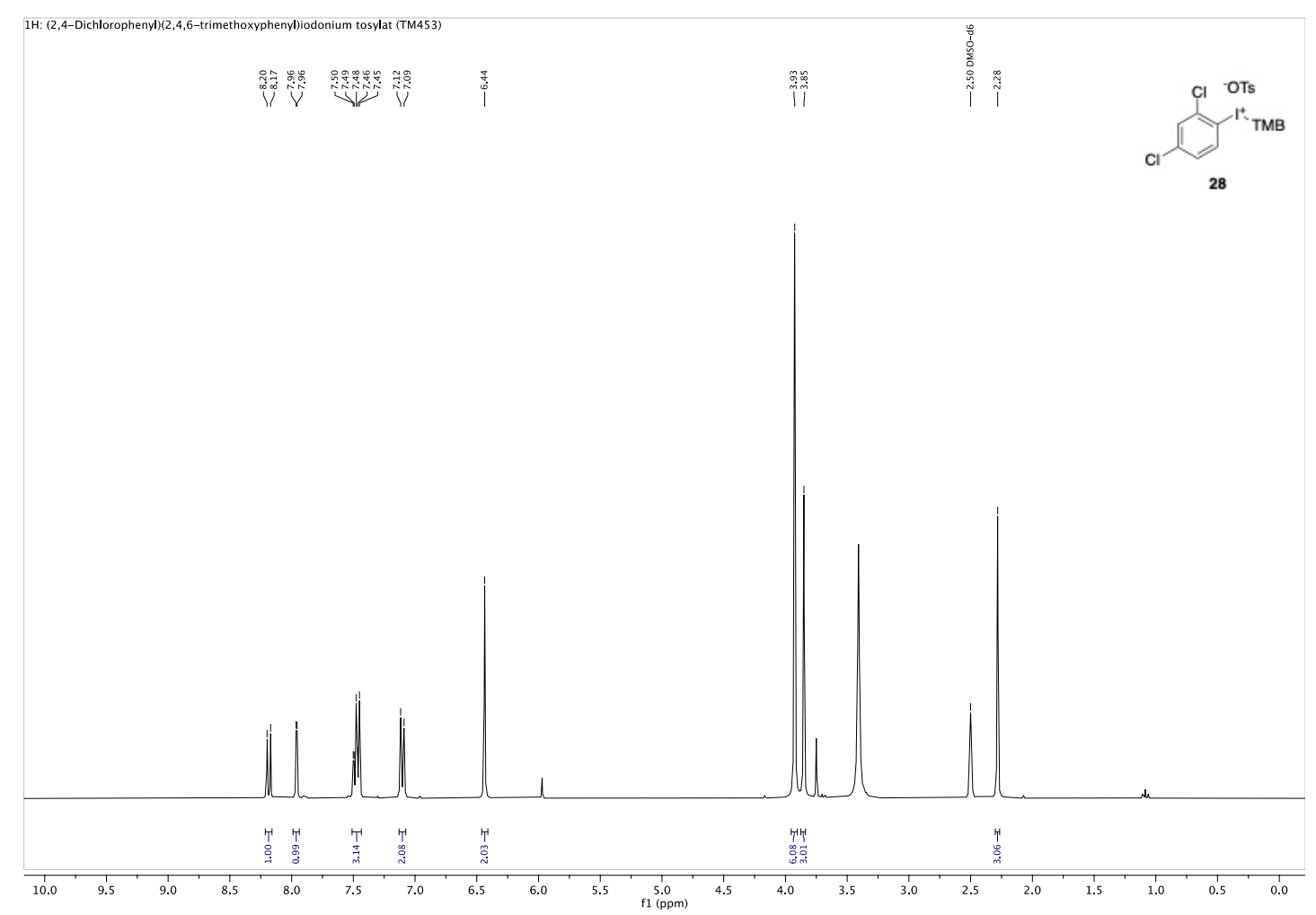

Fig. S42 ${ }^{1} \mathrm{H}$-NMR spectrum of (2,4-dichlorophenyl)(2,4,6-trimethoxyphenyl)iodonium 4methylbenzenesulfonate (28), measured in DMSO-d6 at $75 \mathrm{MHz}$.

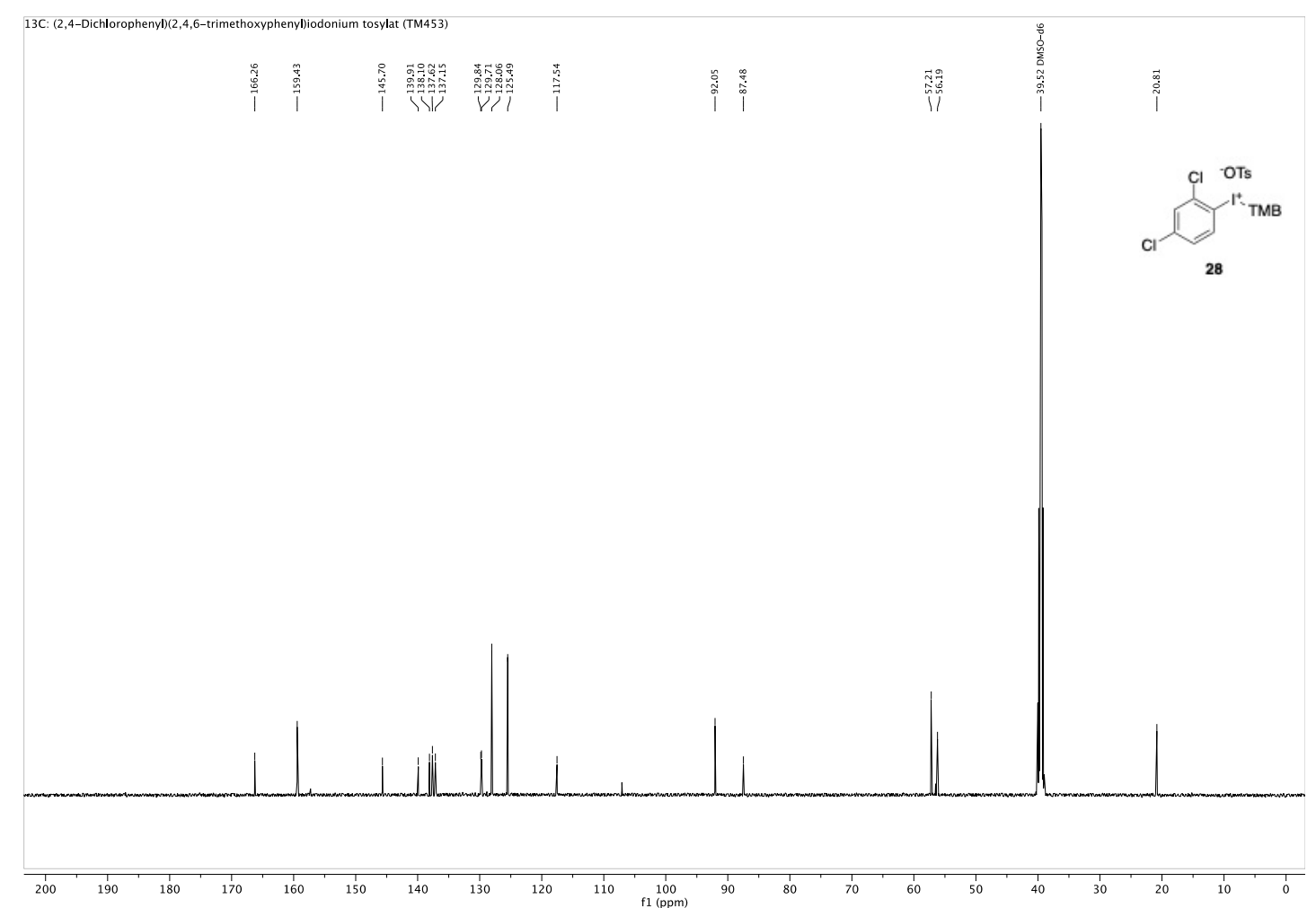

Fig. S43 ${ }^{13} \mathrm{C}$-NMR spectrum of (2,4-dichlorophenyl)(2,4,6-trimethoxyphenyl)iodonium 4methylbenzenesulfonate (28), measured in DMSO- $\mathrm{d}_{6}$ at $75 \mathrm{MHz}$. 


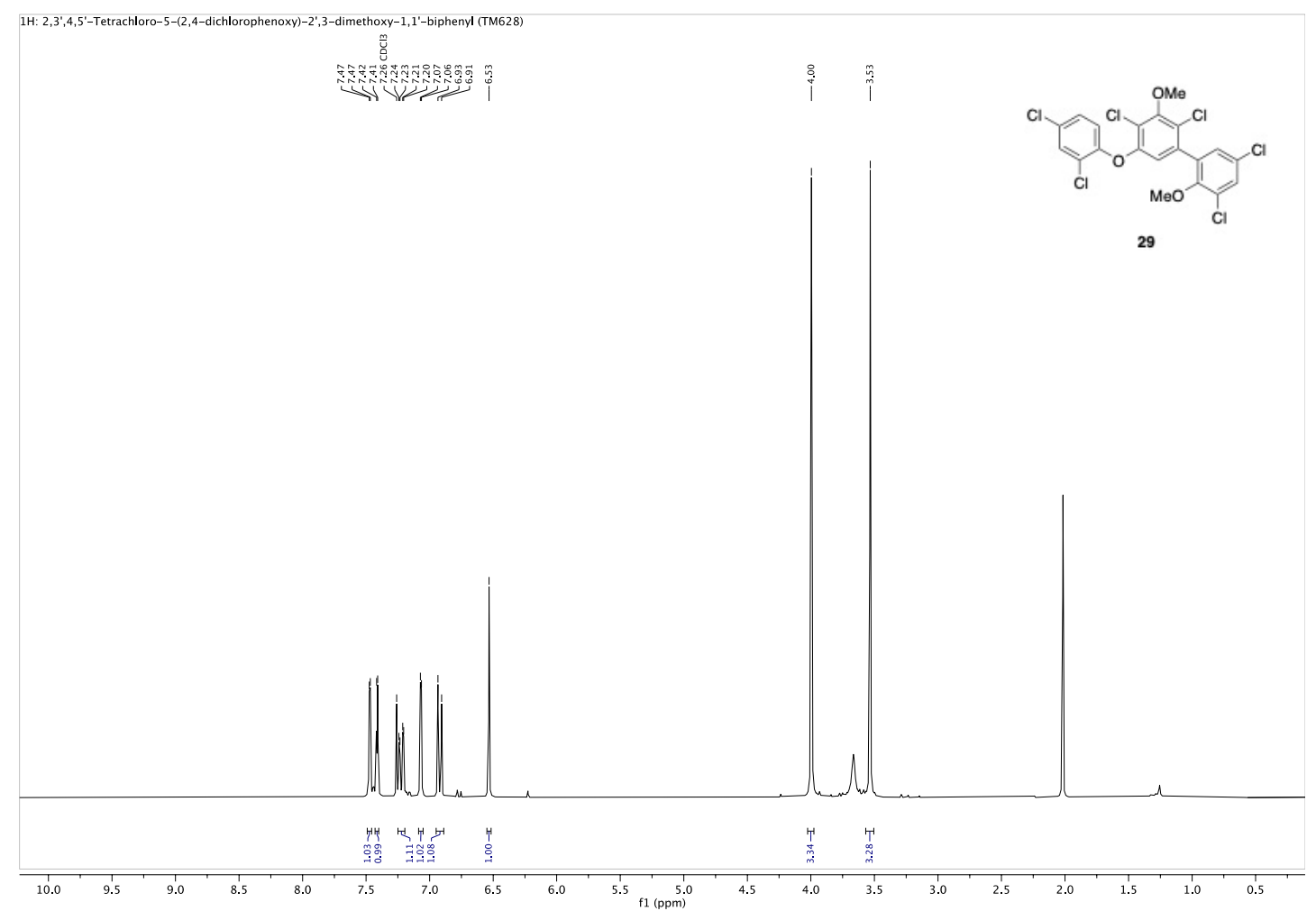

Fig. S44 ${ }^{1} \mathrm{H}-\mathrm{NMR}$ spectrum of 2,3',4,5'-tetrachloro-5-(2,4-dichlorophenoxy)-2',3-dimethoxy-1,1'-biphenyl (29), measured in $\mathrm{CDCl}_{3}$ at $300 \mathrm{MHz}$.

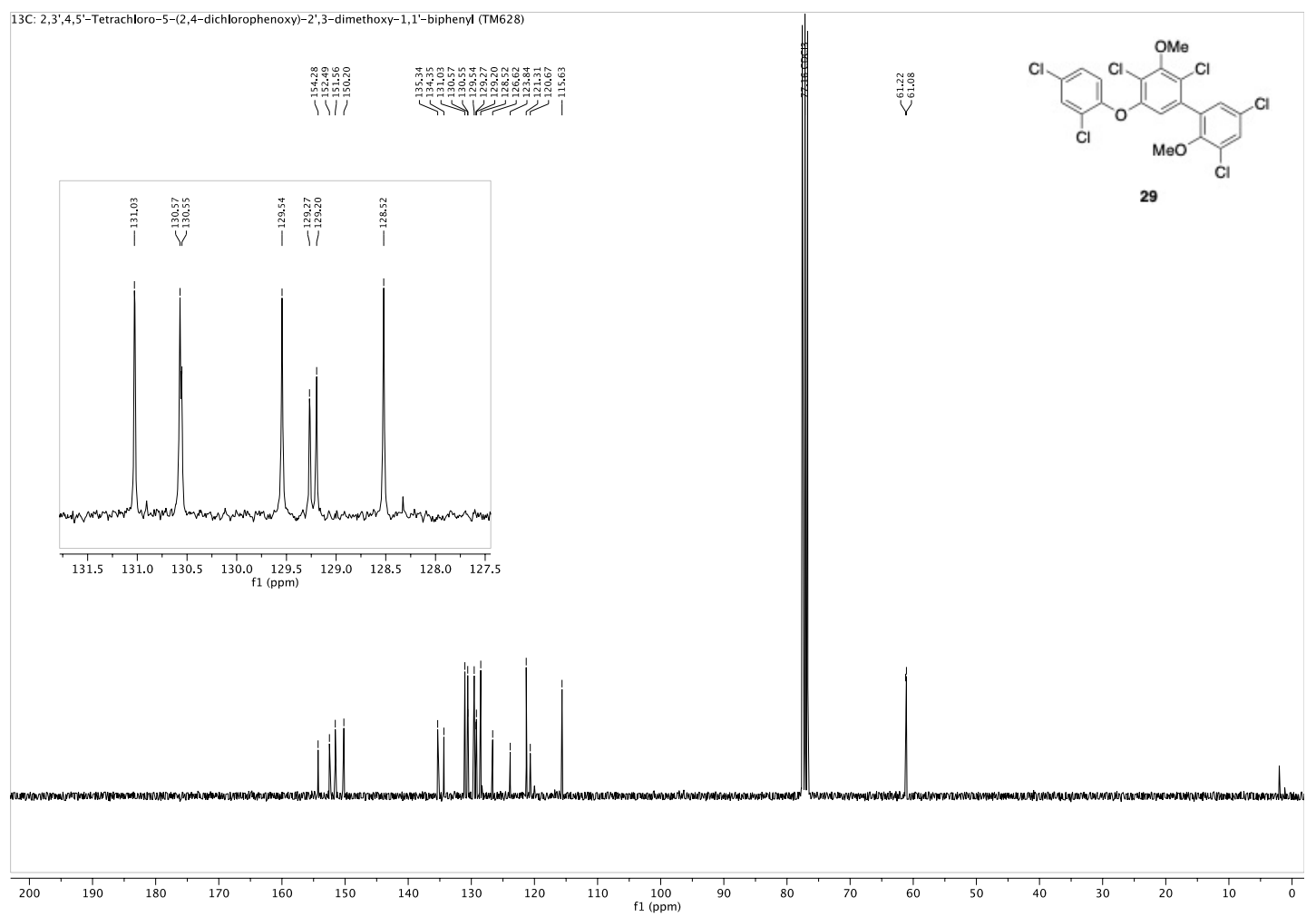

Fig. S45 ${ }^{13} \mathrm{C}-N M R$ spectrum of 2,3',4,5'-tetrachloro-5-(2,4-dichlorophenoxy)-2',3-dimethoxy-1,1'-biphenyl (29), measured in $\mathrm{CDCl}_{3}$ at $75 \mathrm{MHz}$. 


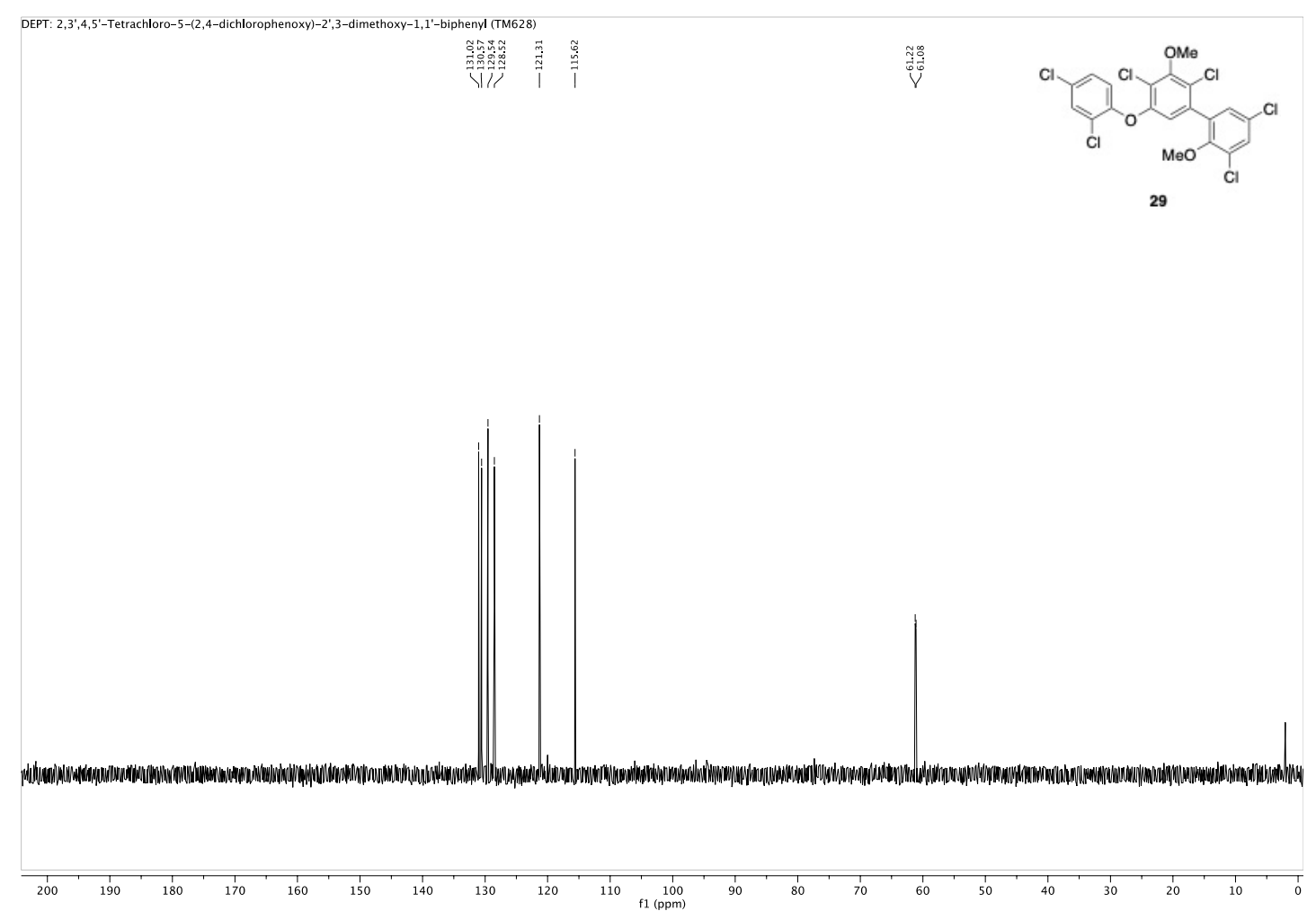

Fig. S46 DEPT spectrum of 2,3',4,5'-tetrachloro-5-(2,4-dichlorophenoxy)-2',3-dimethoxy-1,1'-biphenyl (29), measured in $\mathrm{CDCl}_{3}$ at $75 \mathrm{MHz}$. 


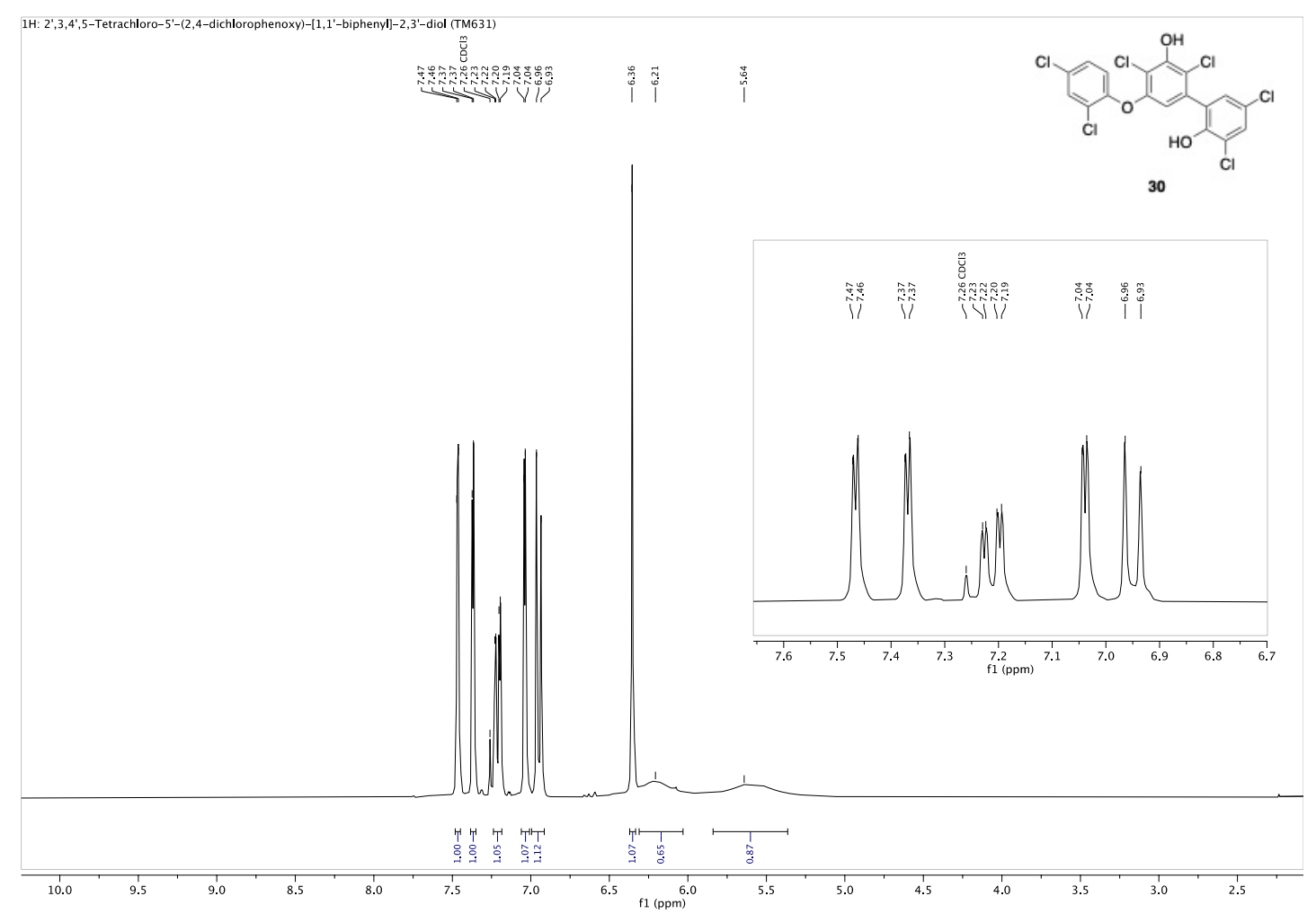

Fig. S47 ${ }^{1} \mathrm{H}$-NMR spectrum of 2',3,4',5-tetrachloro-5'-(2,4-dichlorophenoxy)-[1,1'-biphenyl]-2,3'-diol (30), measured in $\mathrm{CDCl}_{3}$ at $300 \mathrm{MHz}$.

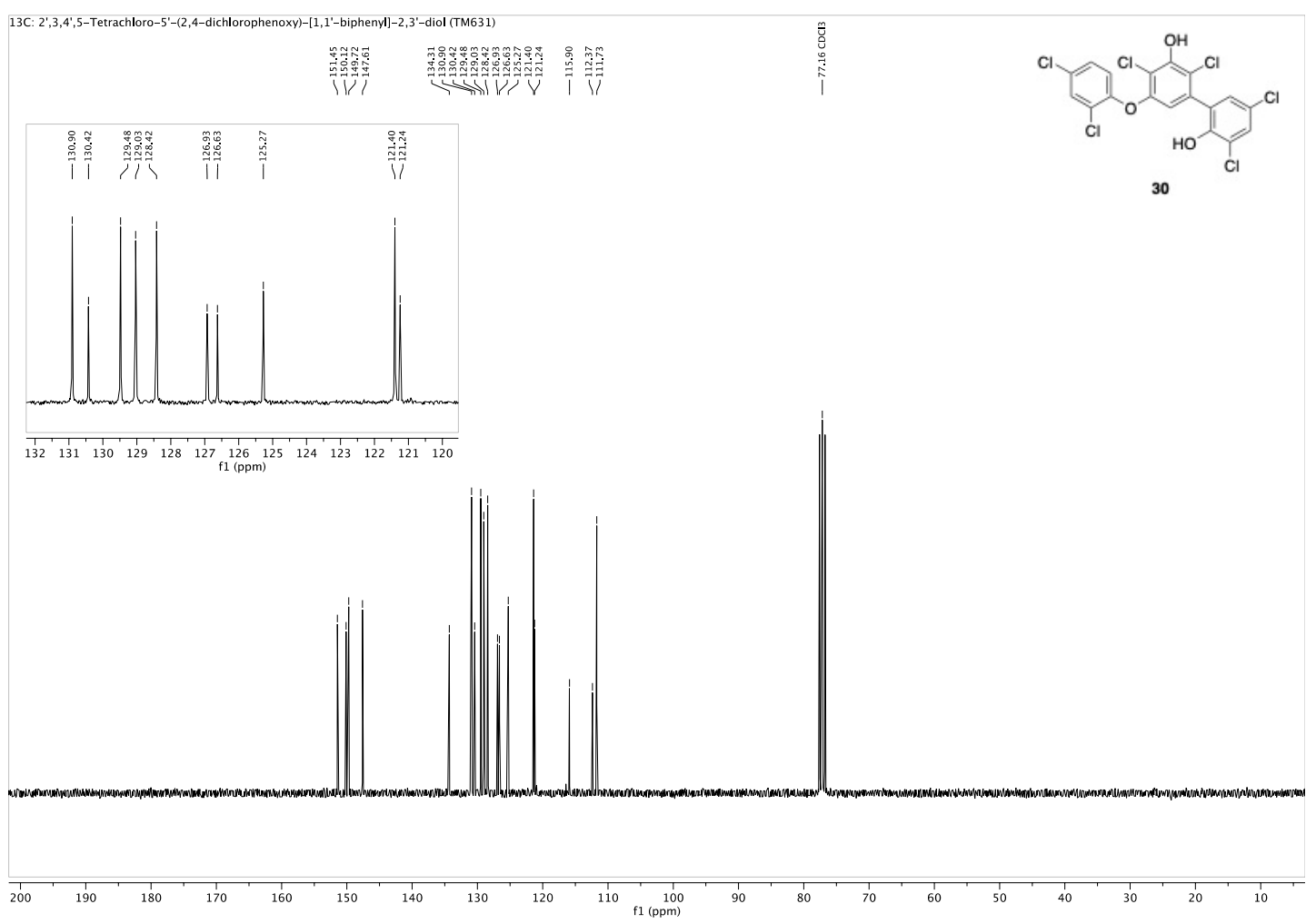

Fig. S48 ${ }^{13}$ C-NMR spectrum of 2',3,4',5-tetrachloro-5'-(2,4-dichlorophenoxy)-[1,1'-biphenyl]-2,3'-diol (30), measured in $\mathrm{CDCl}_{3}$ at $75 \mathrm{MHz}$. 


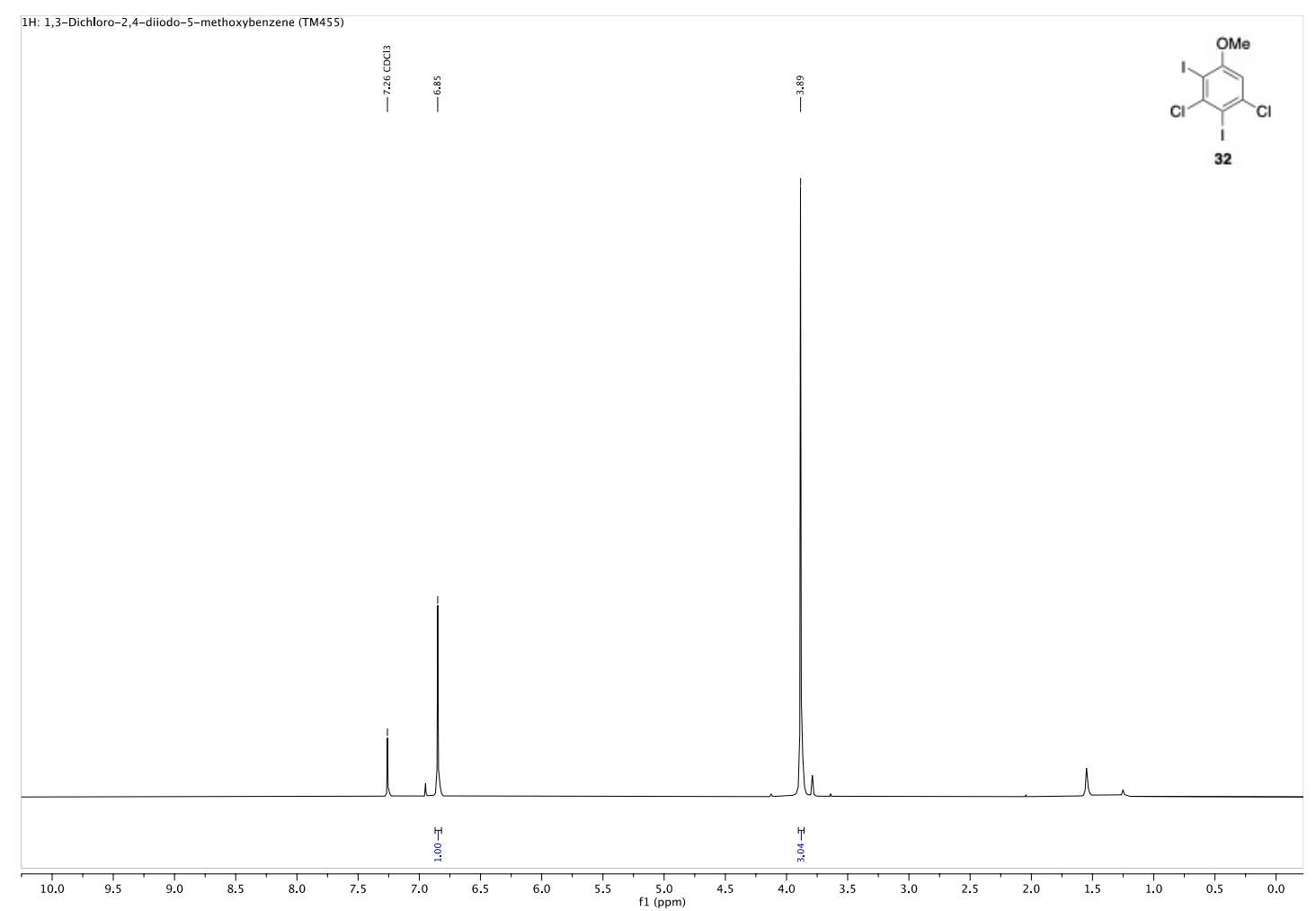

Fig. S49 ${ }^{1} \mathrm{H}-\mathrm{NMR}$ spectrum of 1,3-dichloro-2,4-diiodo-5-methoxybenzene (32), measured in $\mathrm{CDCl}_{3}$ at $300 \mathrm{MHz}$.

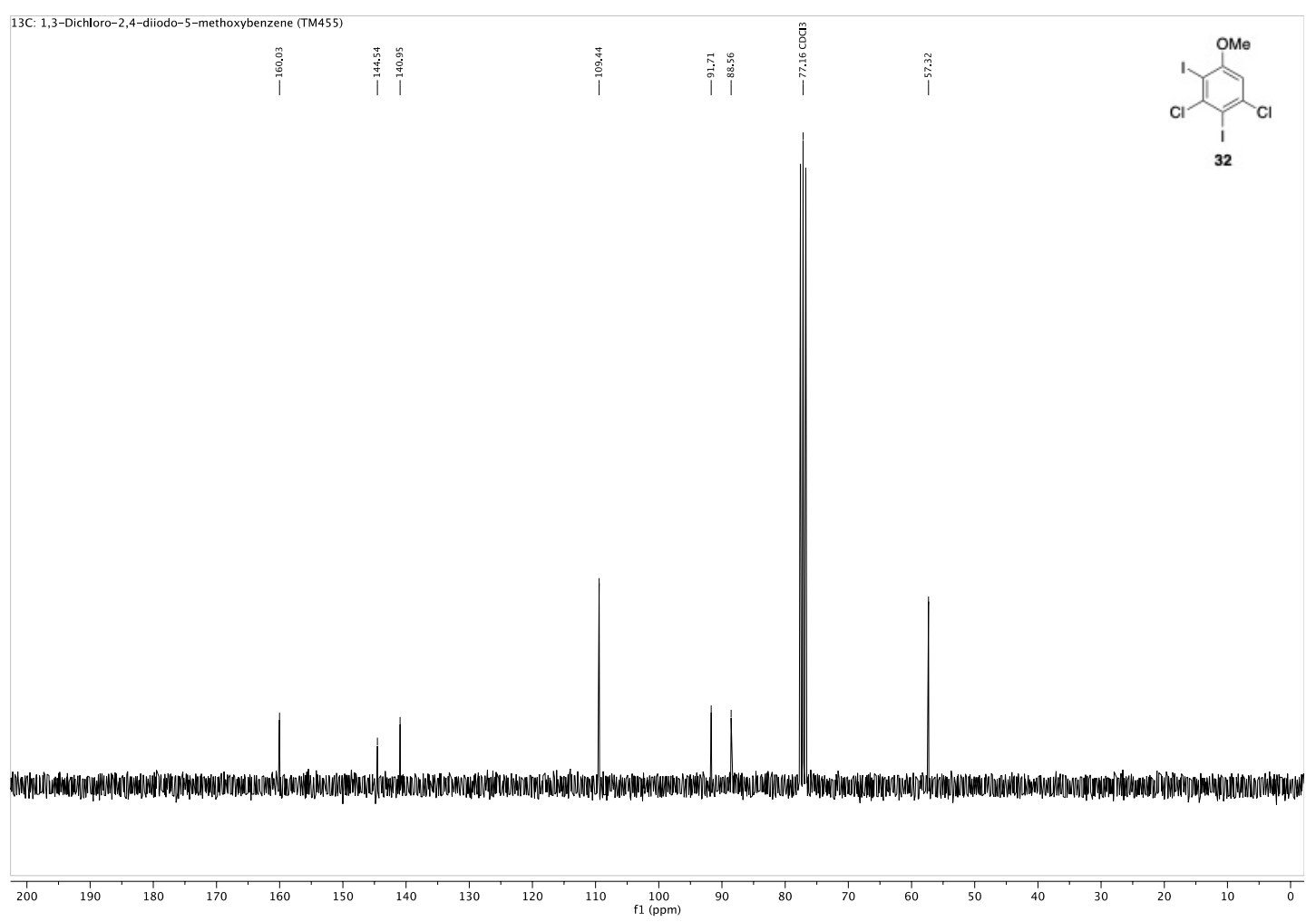

Fig. S50 ${ }^{13} \mathrm{C}-\mathrm{NMR}$ spectrum of 1,3-dichloro-2,4-diiodo-5-methoxybenzene (32), measured in $\mathrm{CDCl}_{3}$ at $75 \mathrm{MHz}$. 


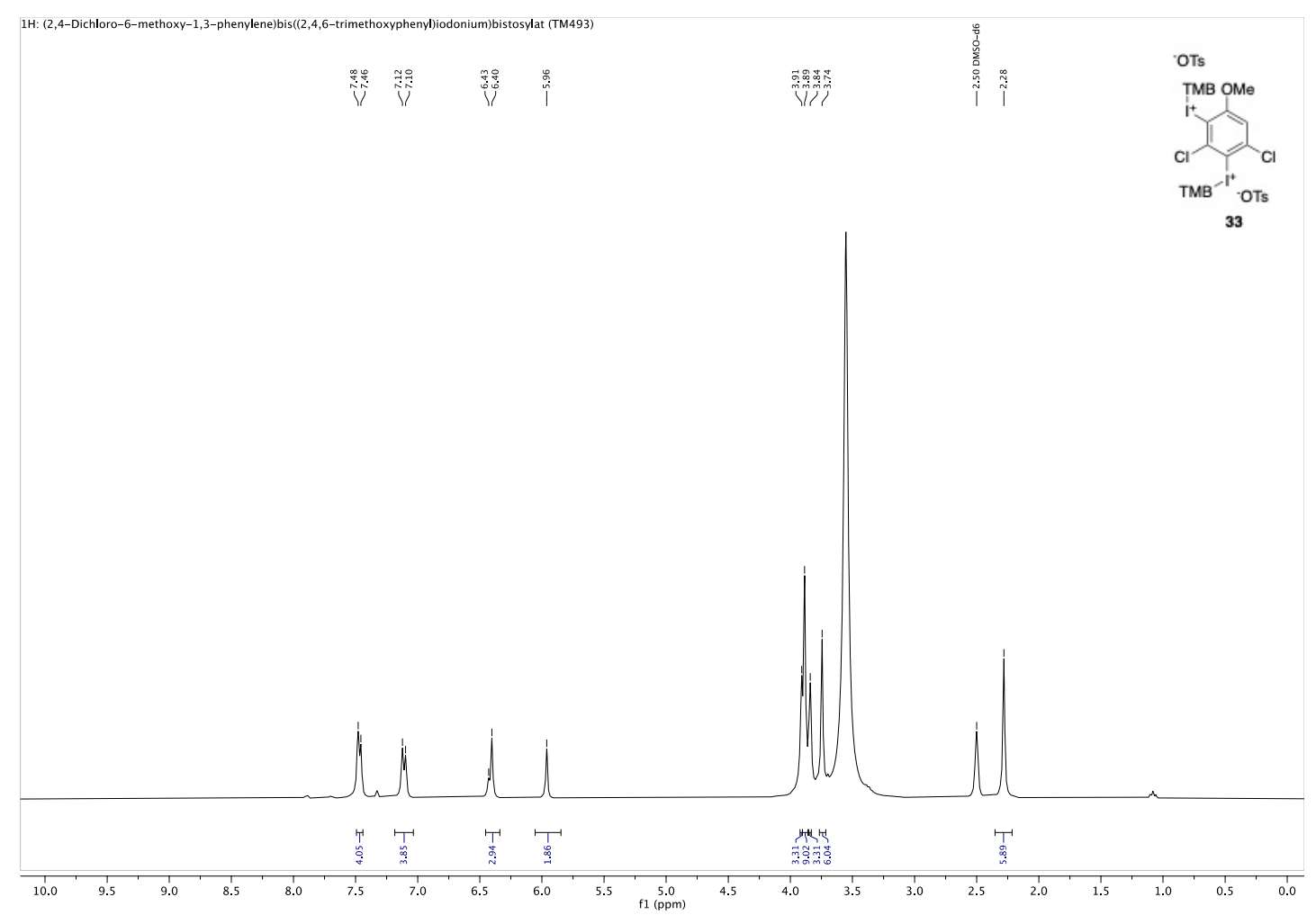

Fig. S51 ${ }^{1} \mathrm{H}$-NMR spectrum of (2,4-dichloro-6-methoxy-1,3-phenylene)bis((2,4,6-trimethoxyphenyl)iodonium) bistosylat (33), measured in DMSO- $\mathrm{d}_{6}$ at $300 \mathrm{MHz}$. 


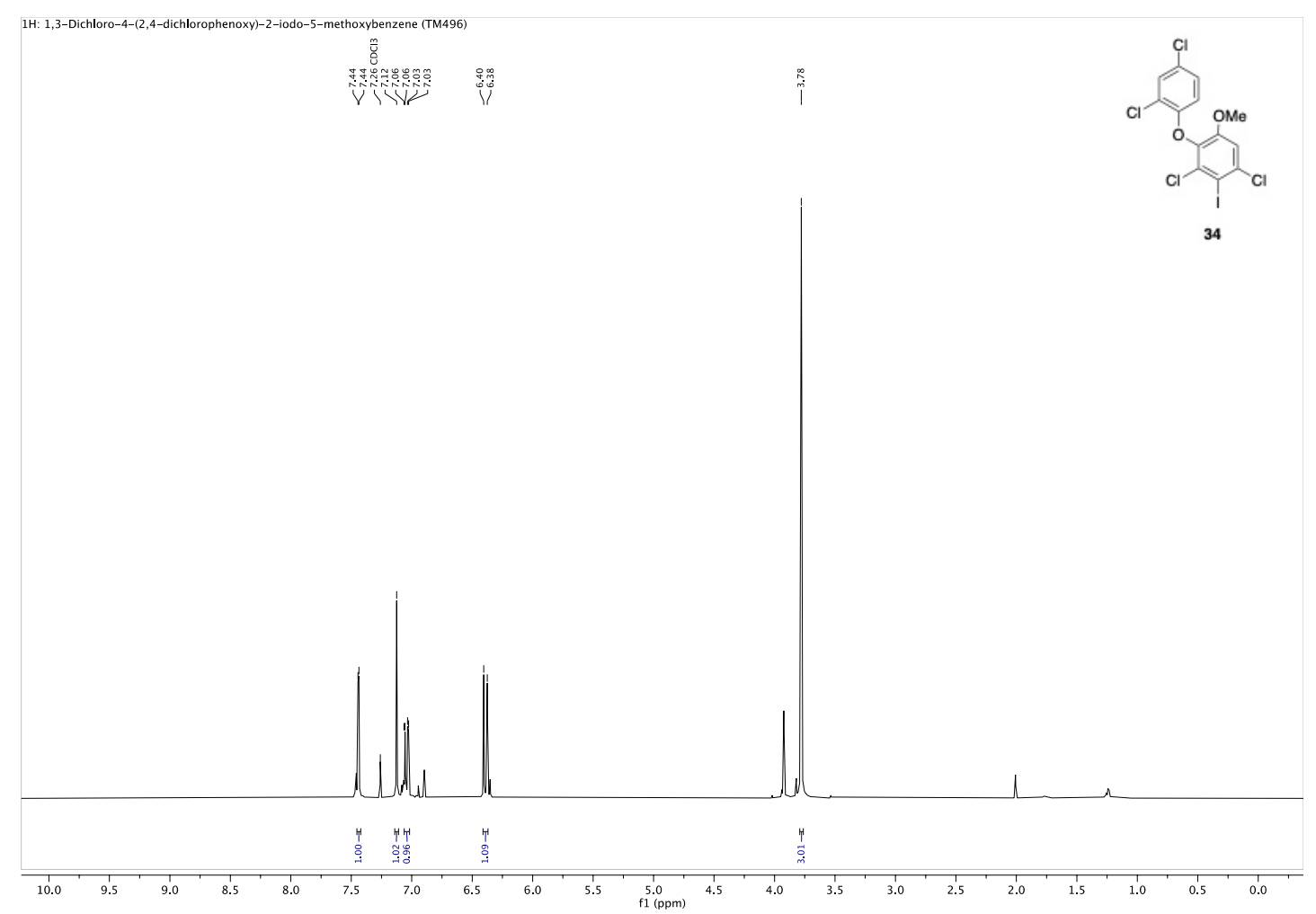

Fig. S52 ${ }^{1} \mathrm{H}-\mathrm{NMR}$ spectrum of 1,3-dichloro-4-(2,4-dichlorophenoxy)-2-iodo-5-methoxybenzene (34), measured in $\mathrm{CDCl}_{3}$ at $300 \mathrm{MHz}$.

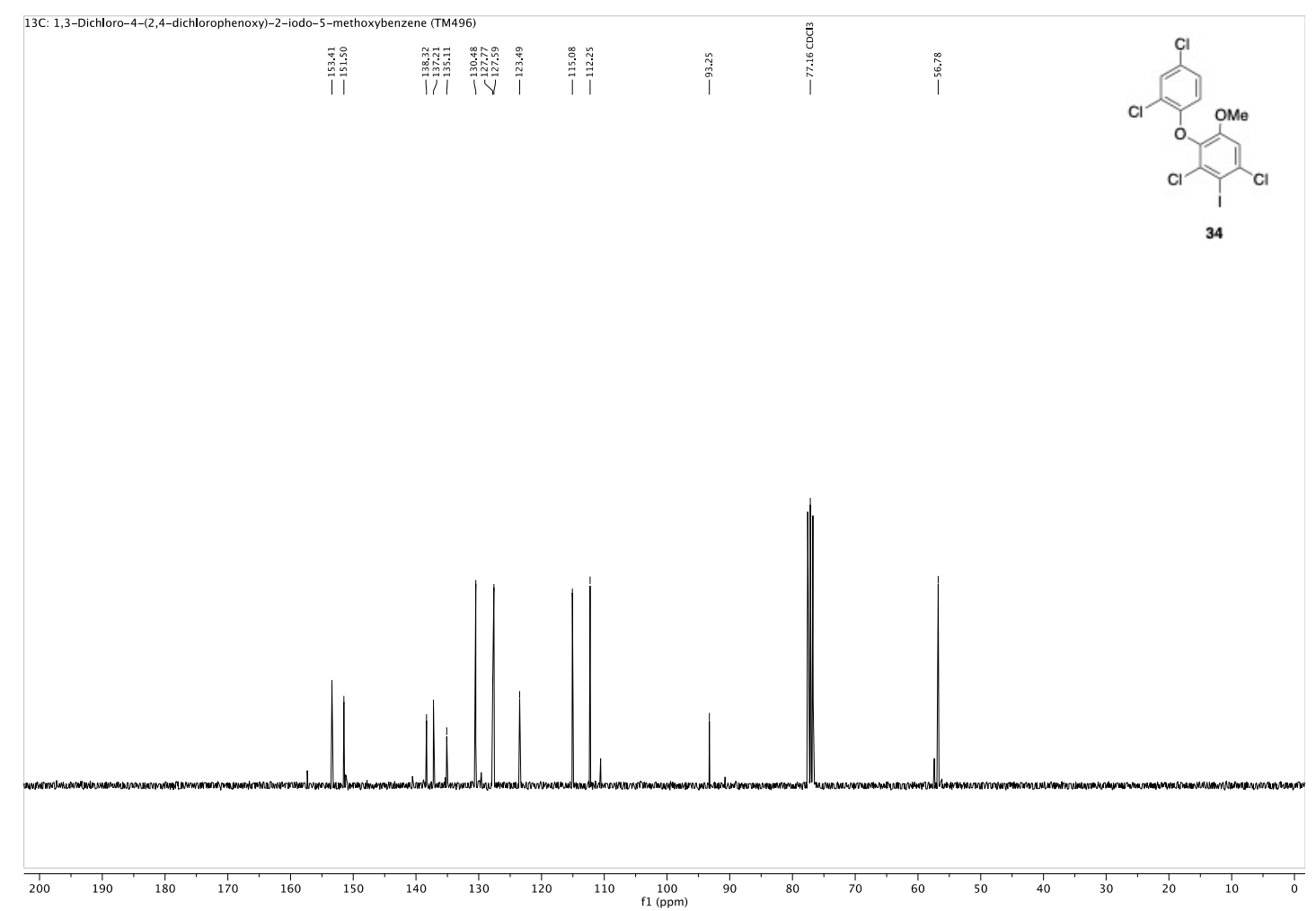

Fig. S53 ${ }^{13}$ C-NMR spectrum of 1,3-dichloro-4-(2,4-dichlorophenoxy)-2-iodo-5-methoxybenzene (34), measured in $\mathrm{CDCl}_{3}$ at $75 \mathrm{MHz}$. 


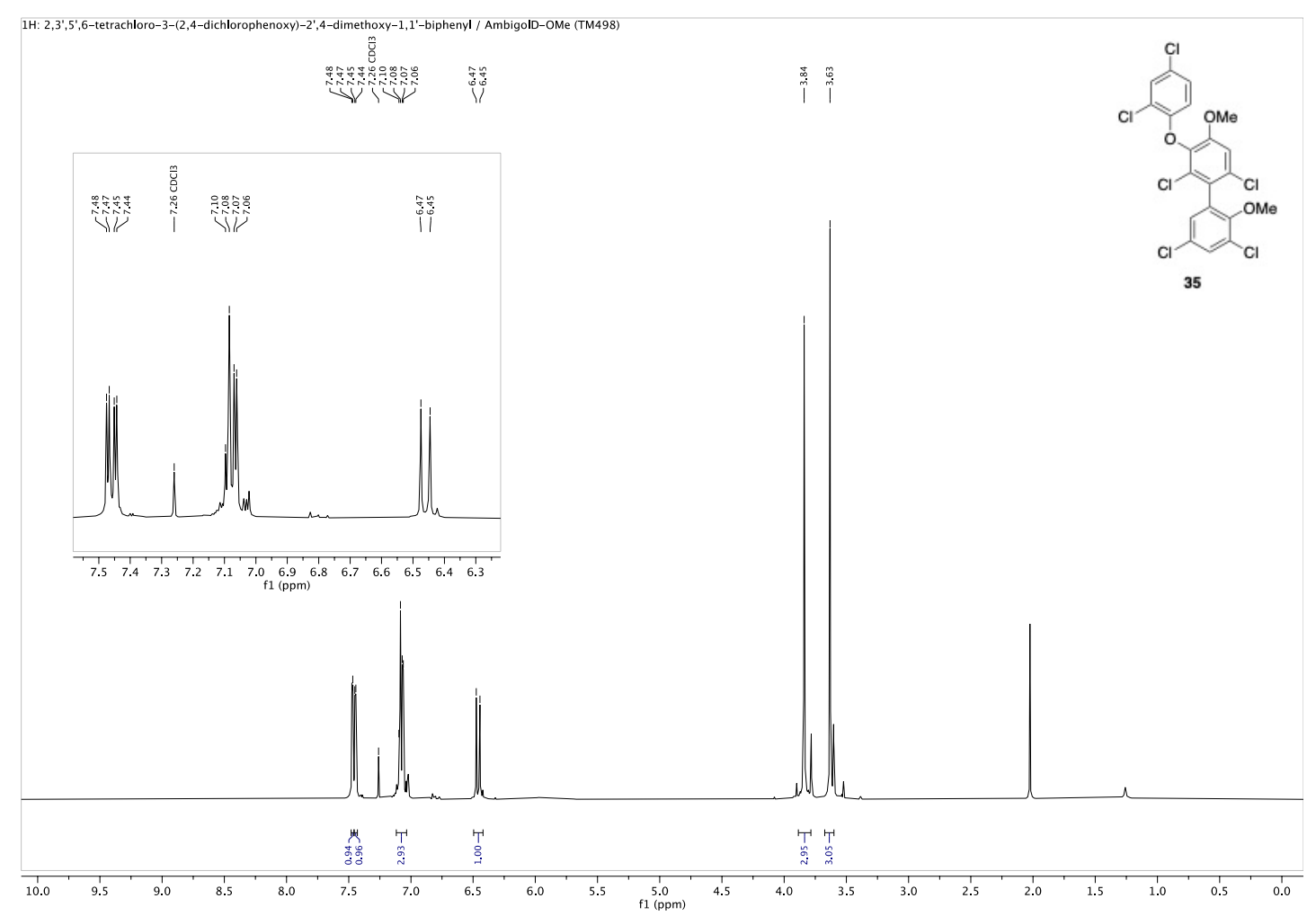

Fig. S54 ${ }^{1} \mathrm{H}-\mathrm{NMR}$ spectrum of 2,3',5',6-tetrachloro-3-(2,4-dichlorophenoxy)-2',4-dimethoxy-1,1'-biphenyl (35), measured in $\mathrm{CDCl}_{3}$ at $300 \mathrm{MHz}$.
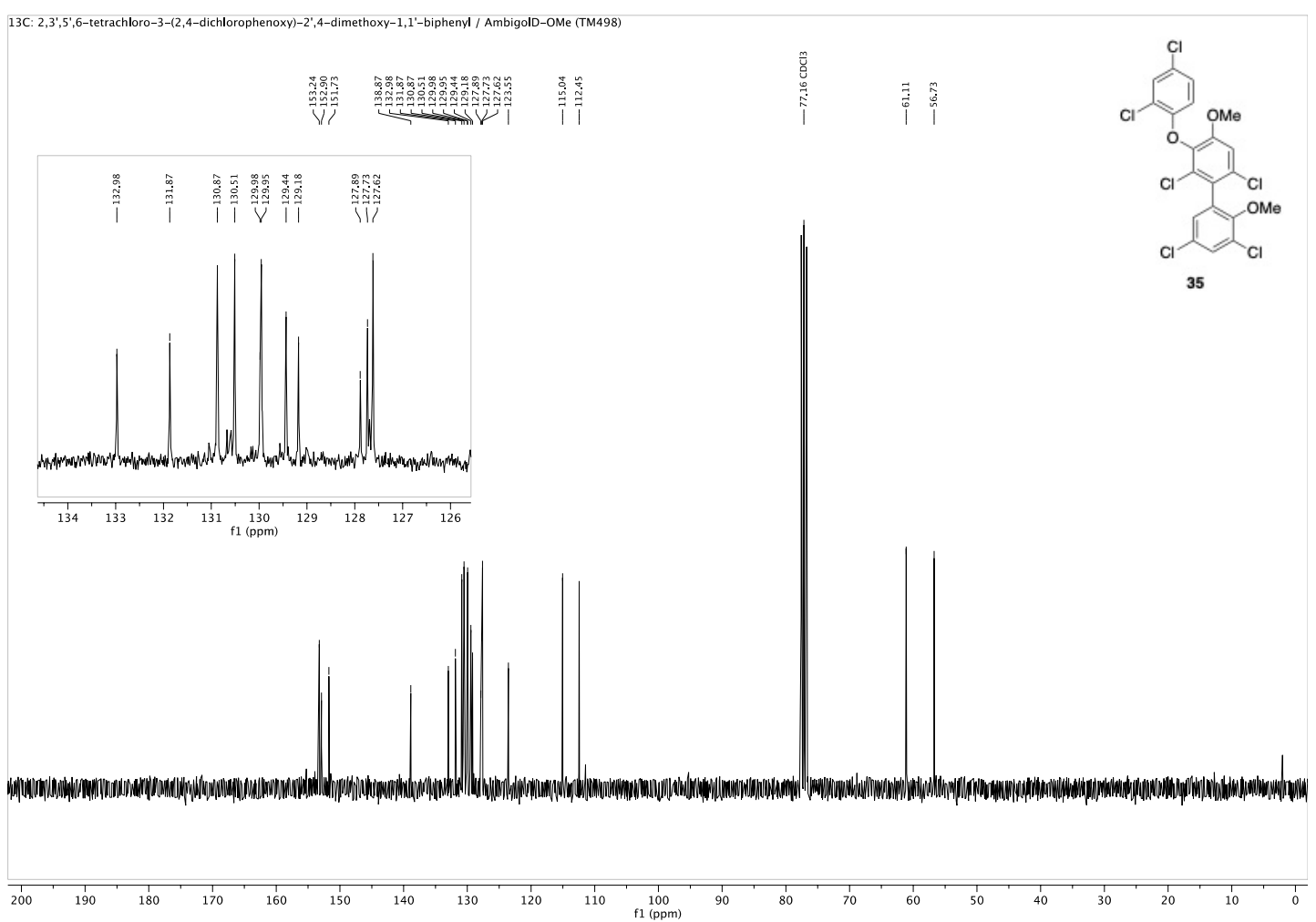

Fig. S55 ${ }^{13} \mathrm{C}$-NMR spectrum of 2,3',5',6-tetrachloro-3-(2,4-dichlorophenoxy)-2',4-dimethoxy-1,1'-biphenyl (35), measured in $\mathrm{CDCl}_{3}$ at $75 \mathrm{MHz}$. 


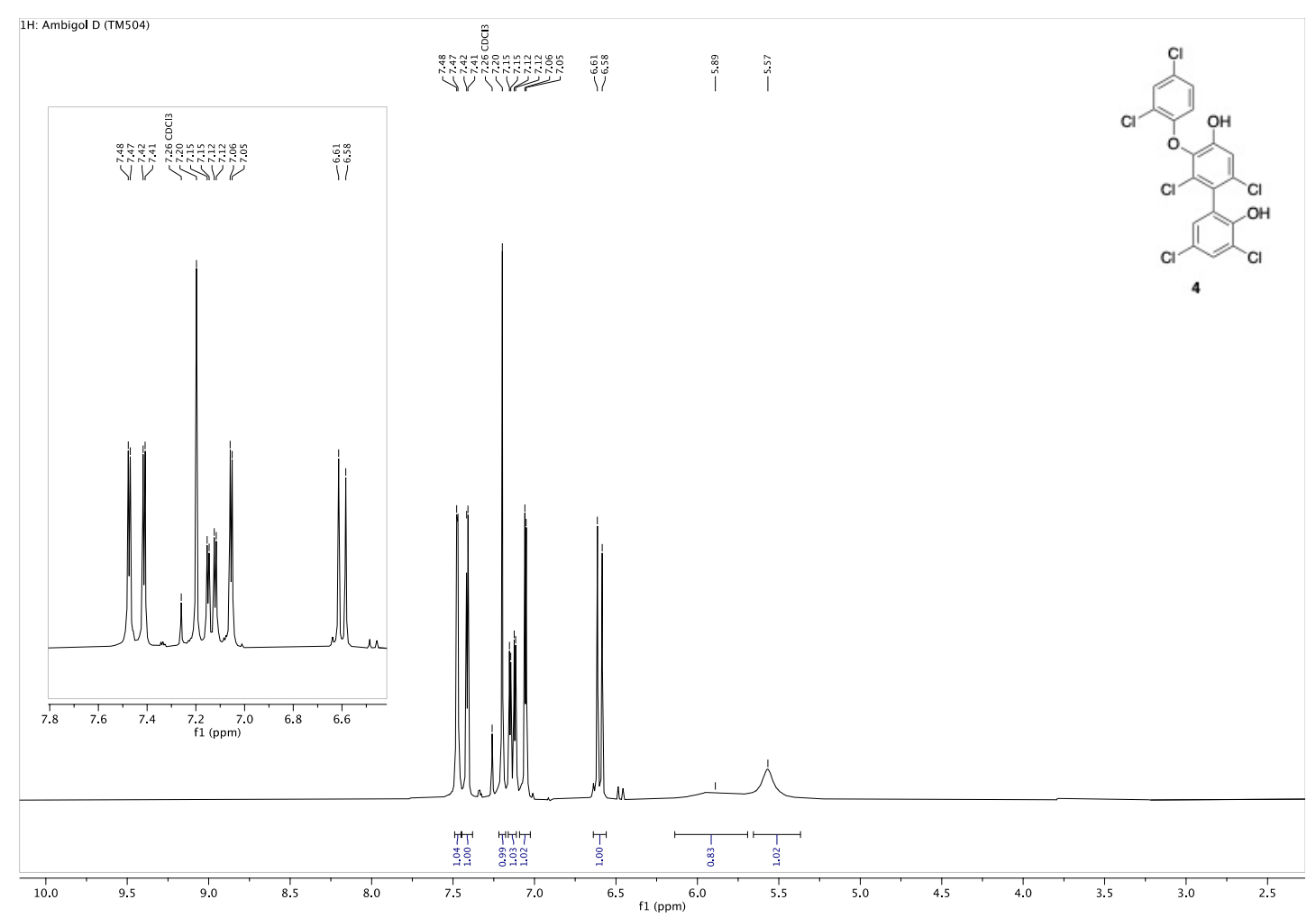

Fig. S56 ${ }^{1} \mathrm{H}-\mathrm{NMR}$ spectrum of ambigol D (4), measured in $\mathrm{CDCl}_{3}$ at $300 \mathrm{MHz}$.
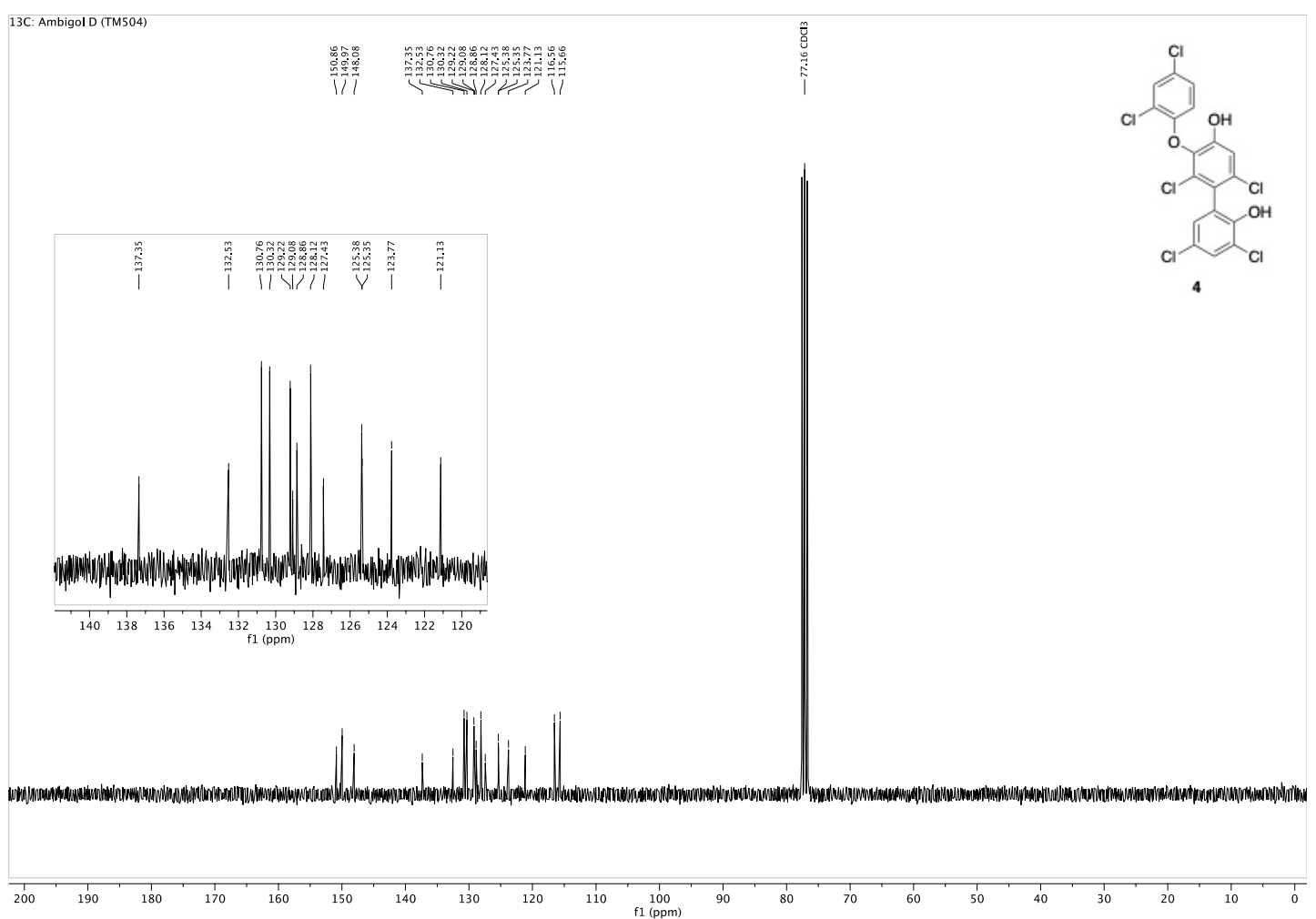

Fig. S57 ${ }^{13} \mathrm{C}-\mathrm{NMR}$ spectrum of ambigol D (4), measured in $\mathrm{CDCl}_{3}$ at $75 \mathrm{MHz}$. 


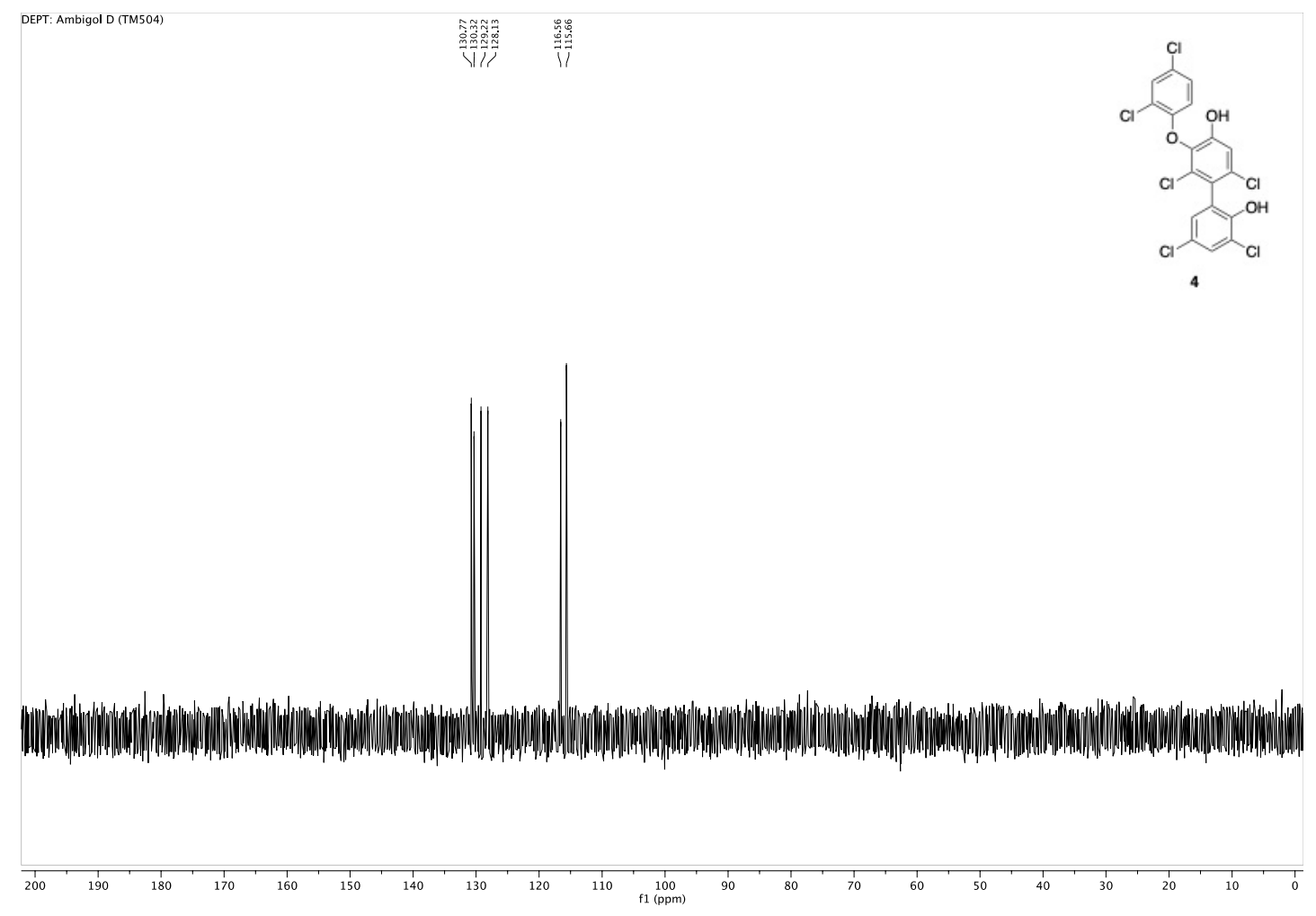

Fig. S58 DEPT spectrum of ambigol D (4), measured in $\mathrm{CDCl}_{3}$ at $75 \mathrm{MHz}$.

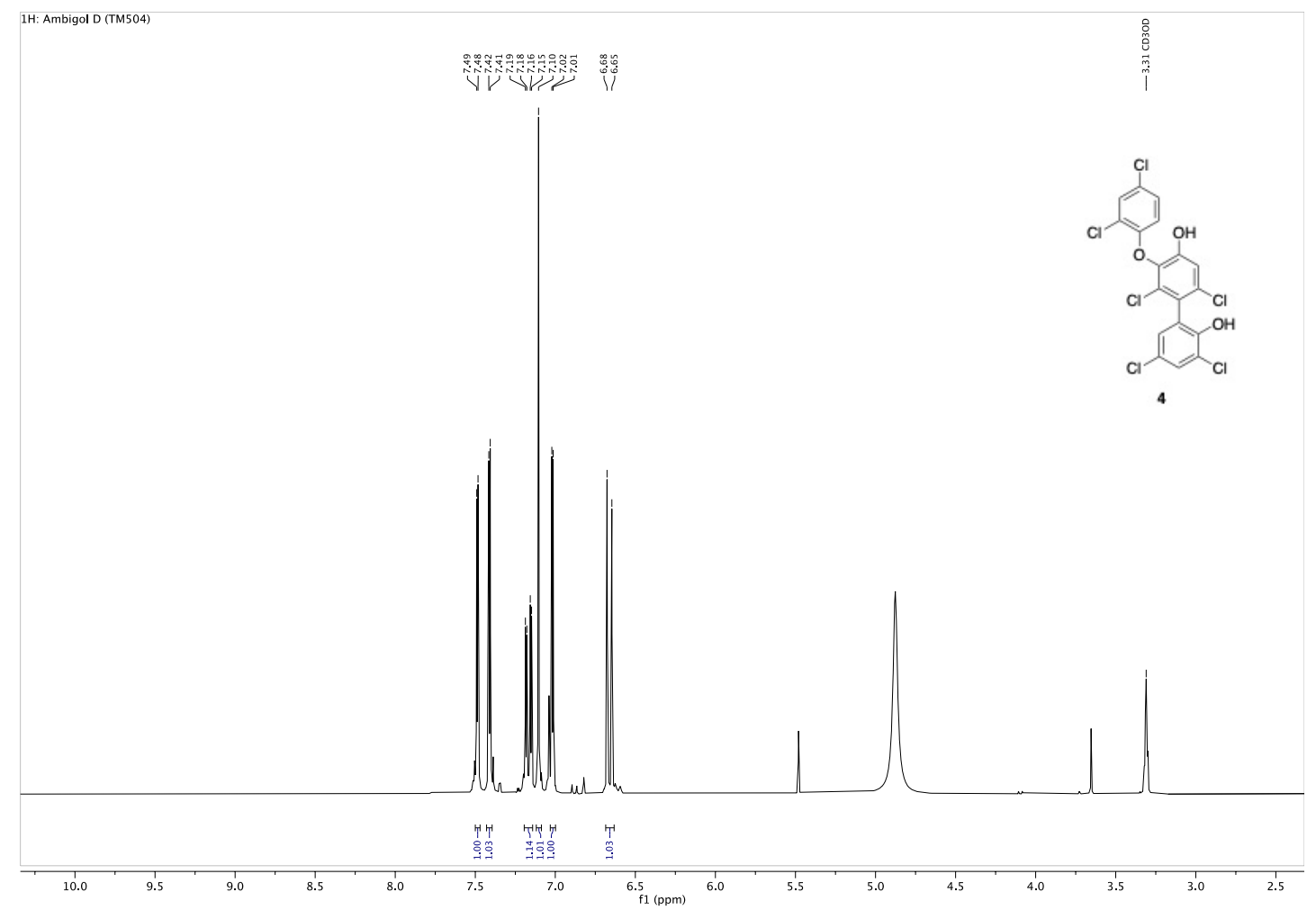

Fig. S59 ${ }^{1} \mathrm{H}-\mathrm{NMR}$ spectrum of ambigol D (4) measured in MeOD-d 4 at $300 \mathrm{MHz}$. 


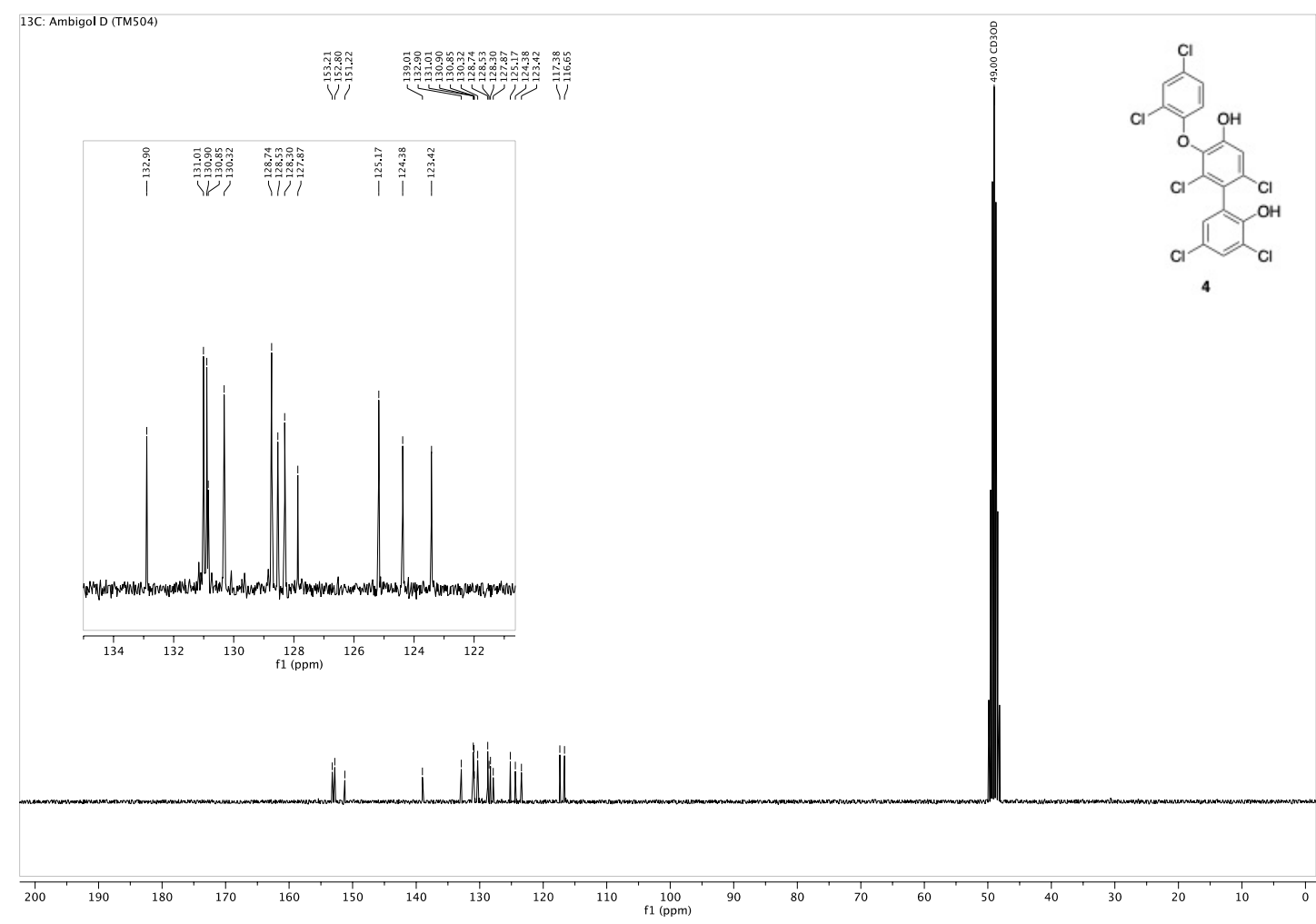

Fig. S60 ${ }^{13} \mathrm{C}-\mathrm{NMR}$ spectrum of ambigol D (4) measured in MeOD-d 4 at $75 \mathrm{MHz}$.

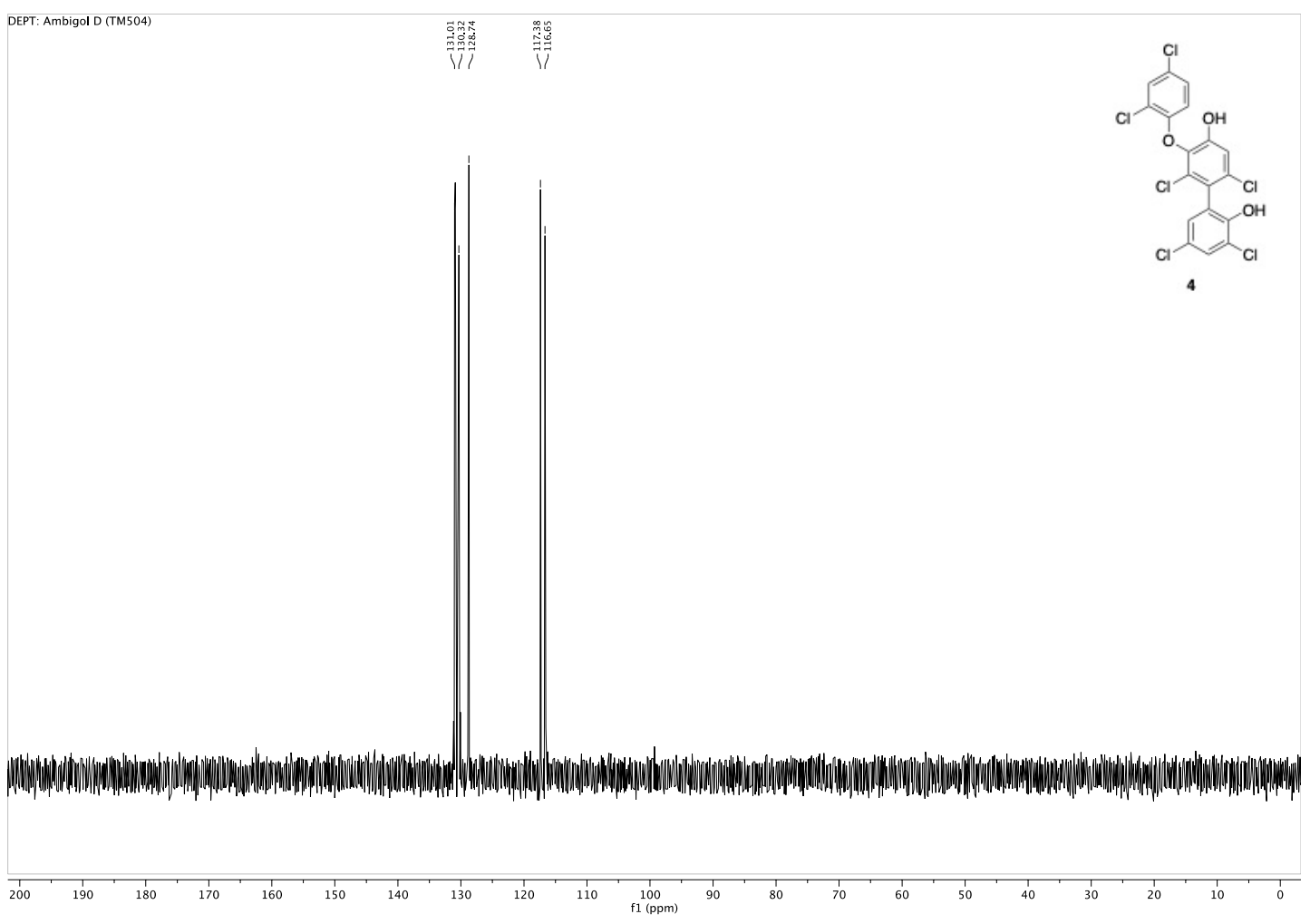

Fig. S61 DEPT spectrum of ambigol D (4) measured in MeOD-d at $75 \mathrm{MHz}$. 


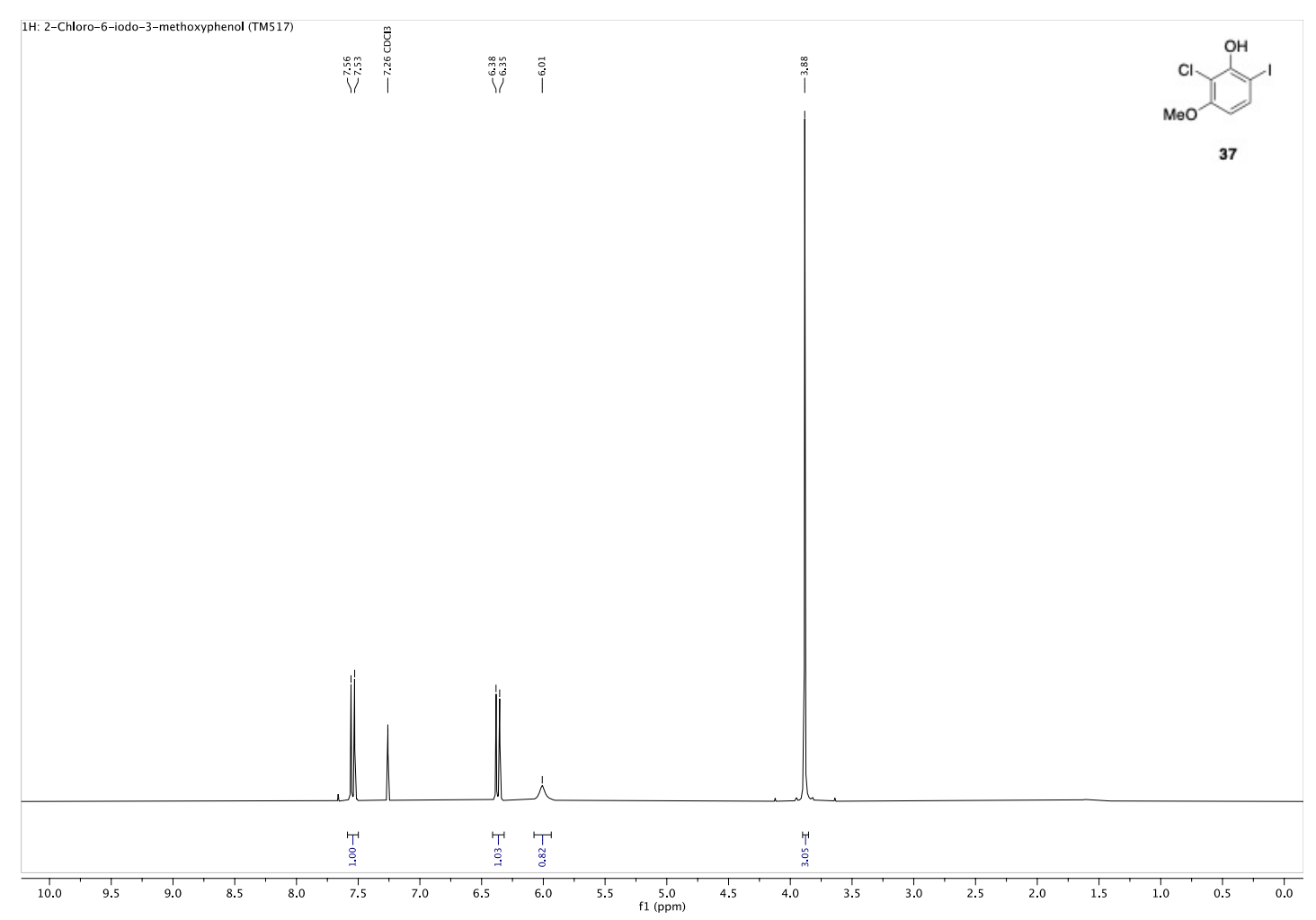

Fig. S62 ${ }^{1} \mathrm{H}-\mathrm{NMR}$ spectrum of 2-chloro-6-iodo-3-methoxyphenol (37), measured in $\mathrm{CDCl}_{3}$ at $300 \mathrm{MHz}$.

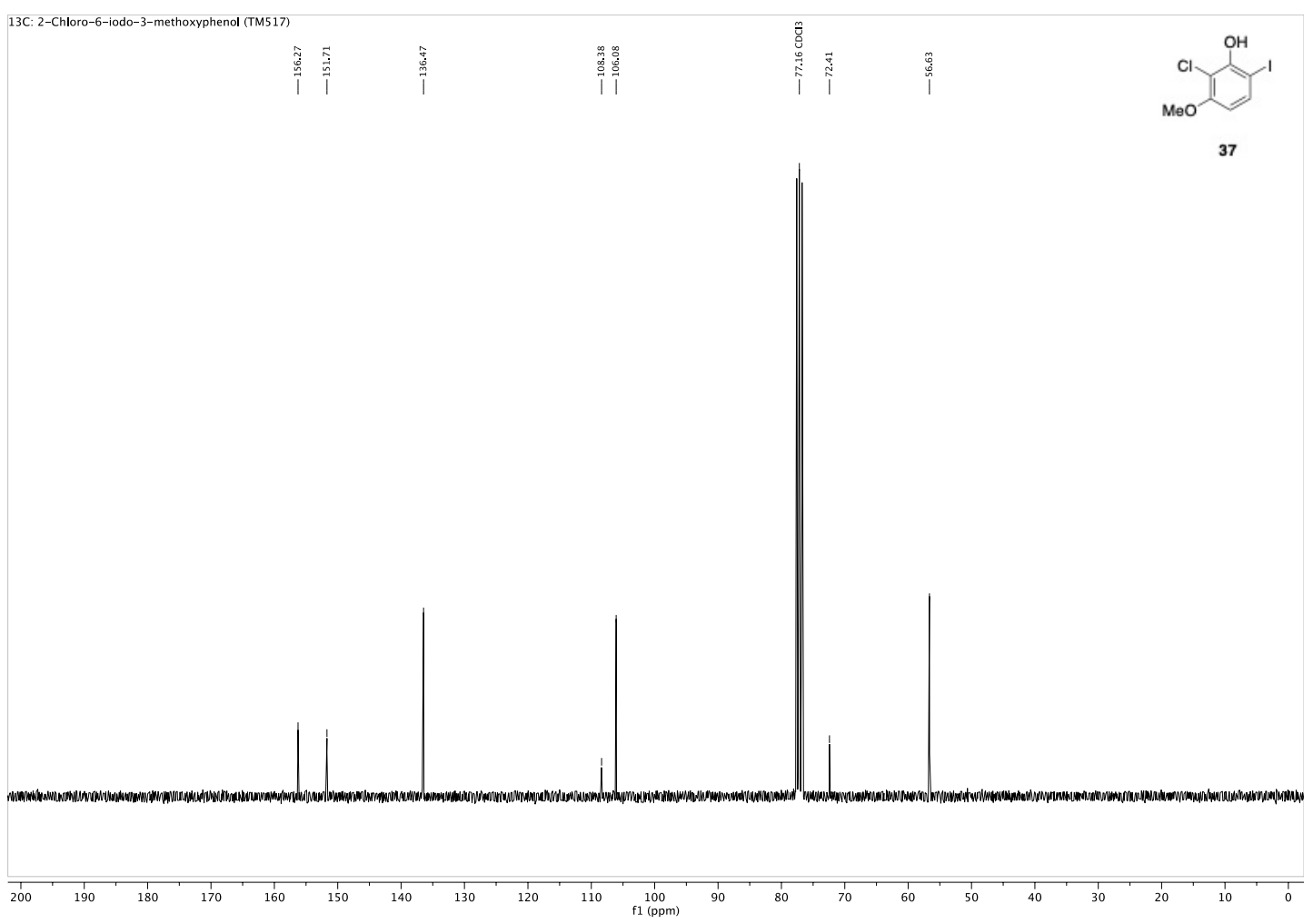

Fig. S63 ${ }^{13} \mathrm{C}-\mathrm{NMR}$ spectrum of 2-chloro-6-iodo-3-methoxyphenol (37), measured in $\mathrm{CDCl}_{3}$ at $75 \mathrm{MHz}$. 


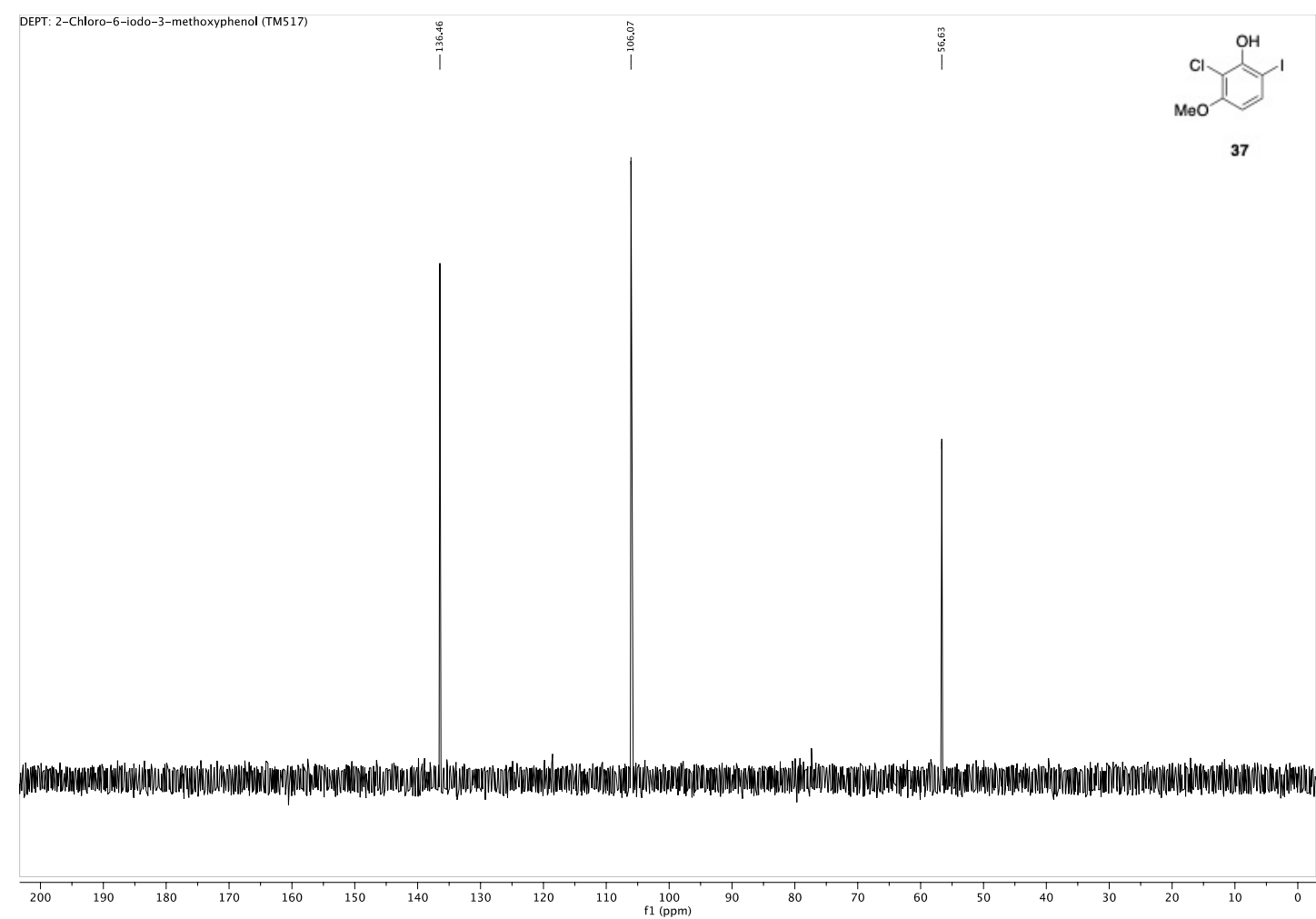

Fig. S64 DEPT spectrum of 2-chloro-6-iodo-3-methoxyphenol (37), measured in $\mathrm{CDCl}_{3}$ at $75 \mathrm{MHz}$. 


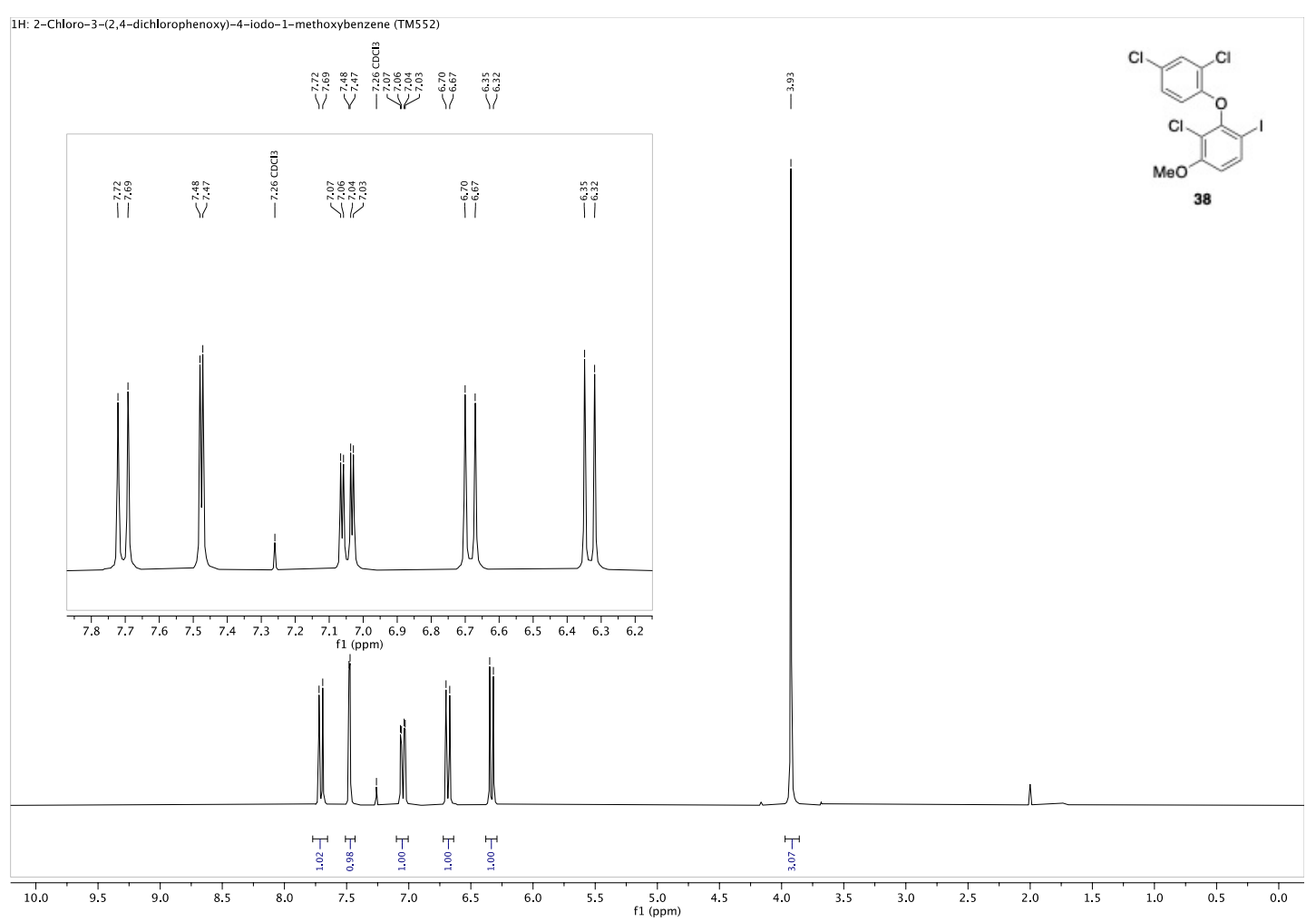

Fig. S65 ${ }^{1} \mathrm{H}-\mathrm{NMR}$ spectrum of 2-chloro-3-(2,4-dichlorophenoxy)-4-iodo-1-methoxybenzene (38), measured in $\mathrm{CDCl}_{3}$ at $300 \mathrm{MHz}$.

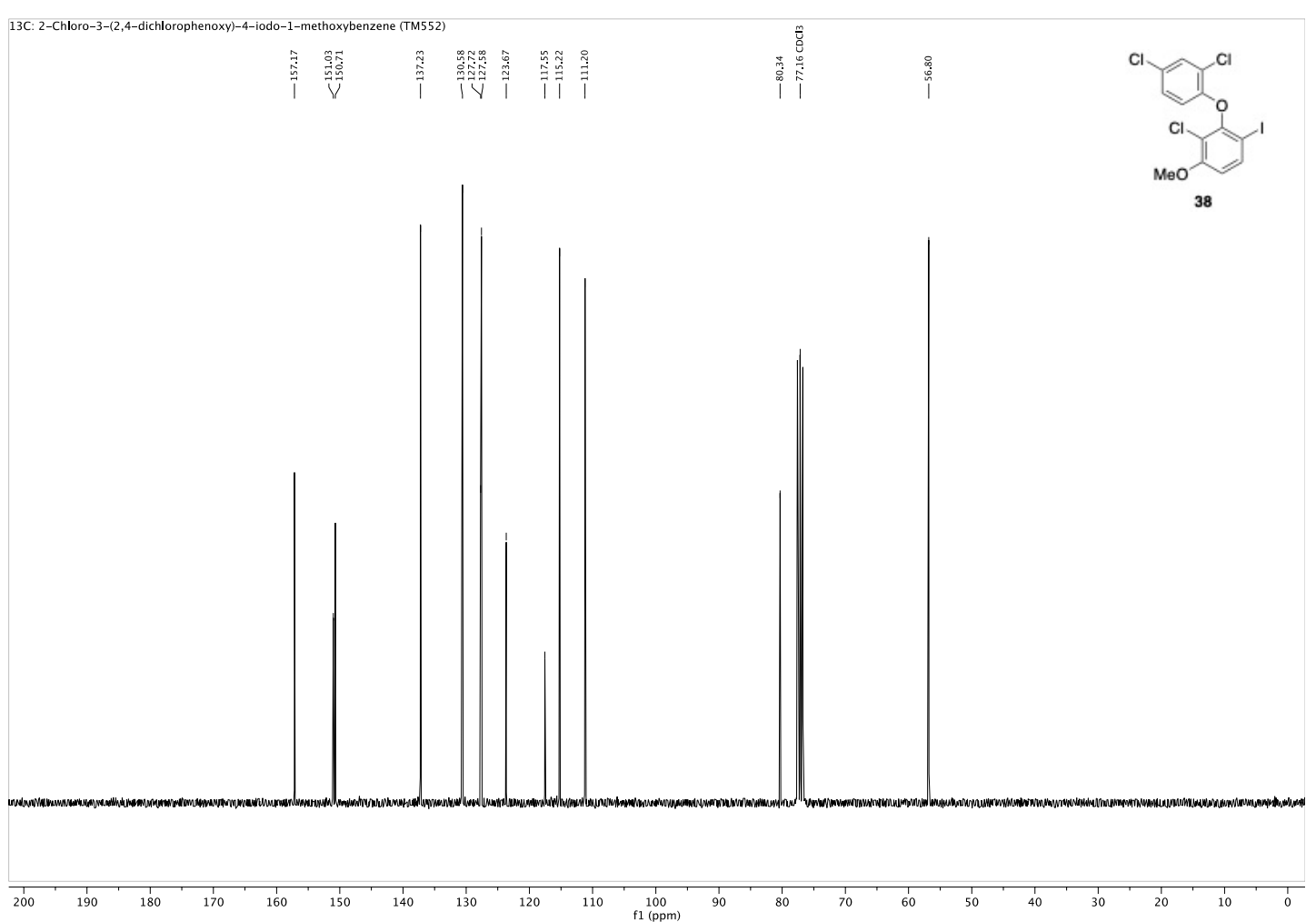

Fig. S66 ${ }^{13}$ C-NMR spectrum of 2-chloro-3-(2,4-dichlorophenoxy)-4-iodo-1-methoxybenzene (38), measured in $\mathrm{CDCl}_{3}$ at $75 \mathrm{MHz}$. 


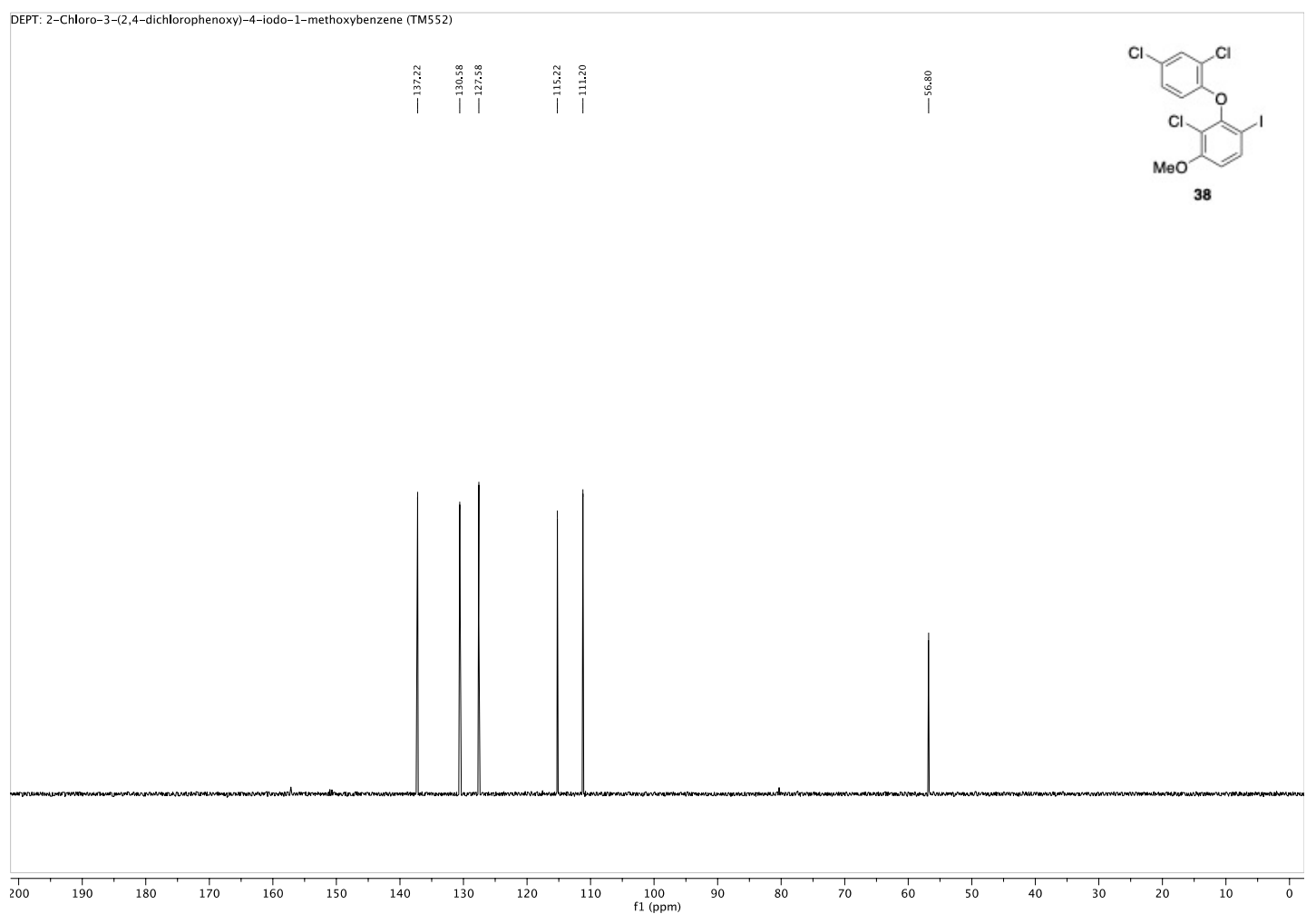

Fig. S67 DEPT spectrum of 2-chloro-3-(2,4-dichlorophenoxy)-4-iodo-1-methoxybenzene (38), measured in $\mathrm{CDCl}_{3}$ at $75 \mathrm{MHz}$. 


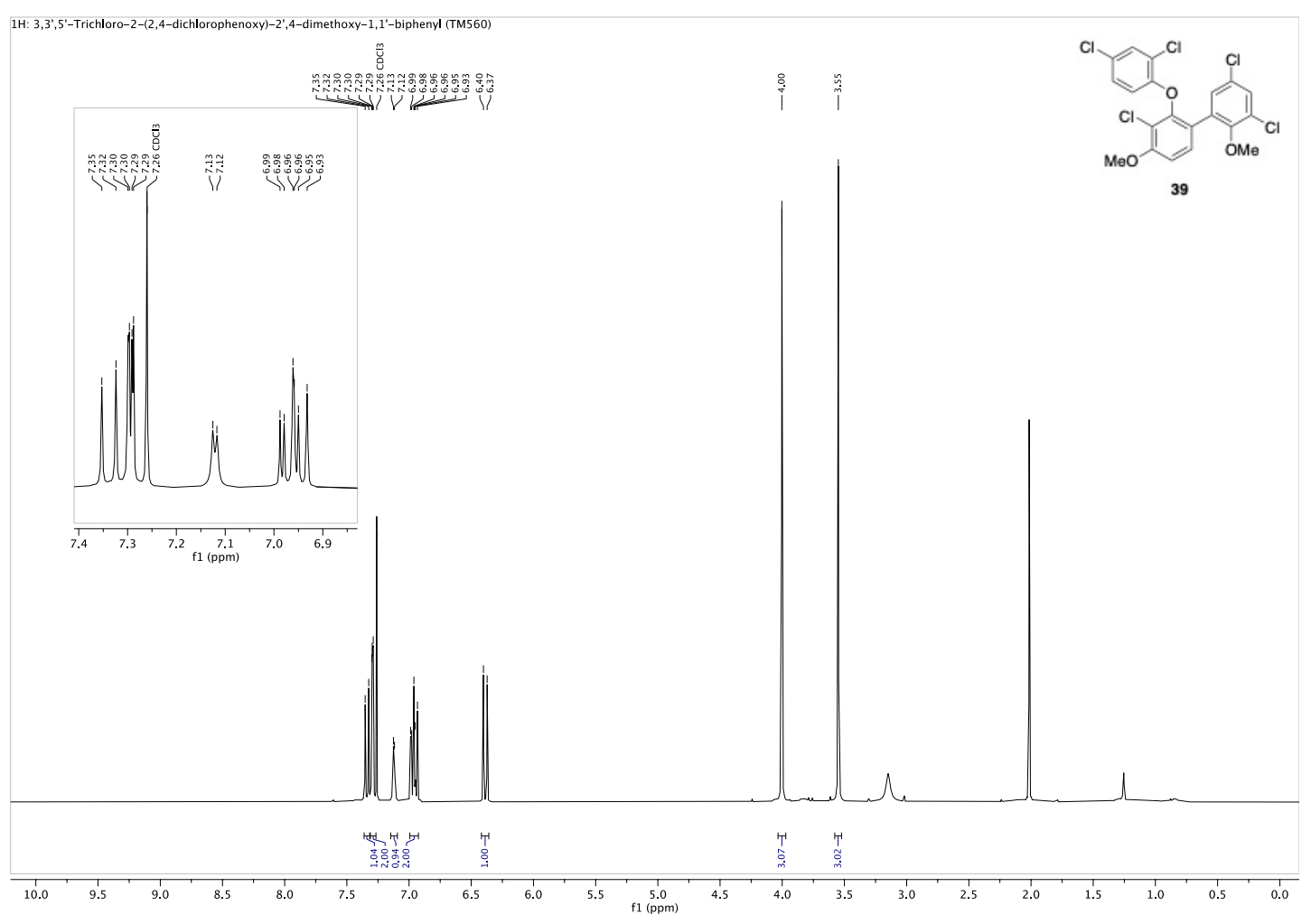

Fig. S68 ${ }^{1} \mathrm{H}-\mathrm{NMR}$ spectrum of 3,3',5'-trichloro-2-(2,4-dichlorophenoxy)-2',4-dimethoxy-1,1'-biphenyl (39), measured in $\mathrm{CDCl}_{3}$ at $300 \mathrm{MHz}$.

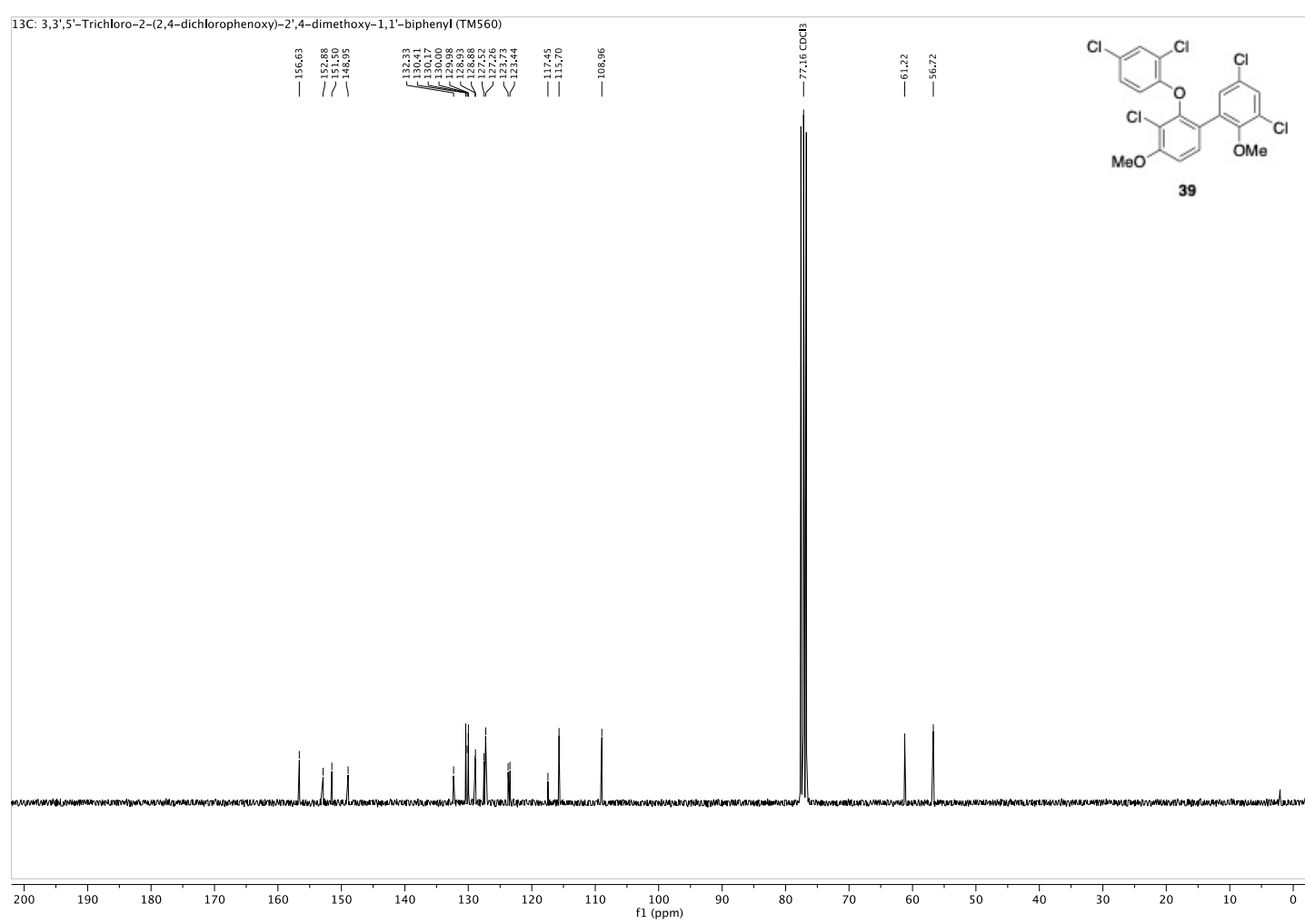

Fig. S69 ${ }^{13}$ C-NMR spectrum of 3,3',5'-trichloro-2-(2,4-dichlorophenoxy)-2',4-dimethoxy-1,1'-biphenyl (39), measured in $\mathrm{CDCl}_{3}$ at $75 \mathrm{MHz}$. 


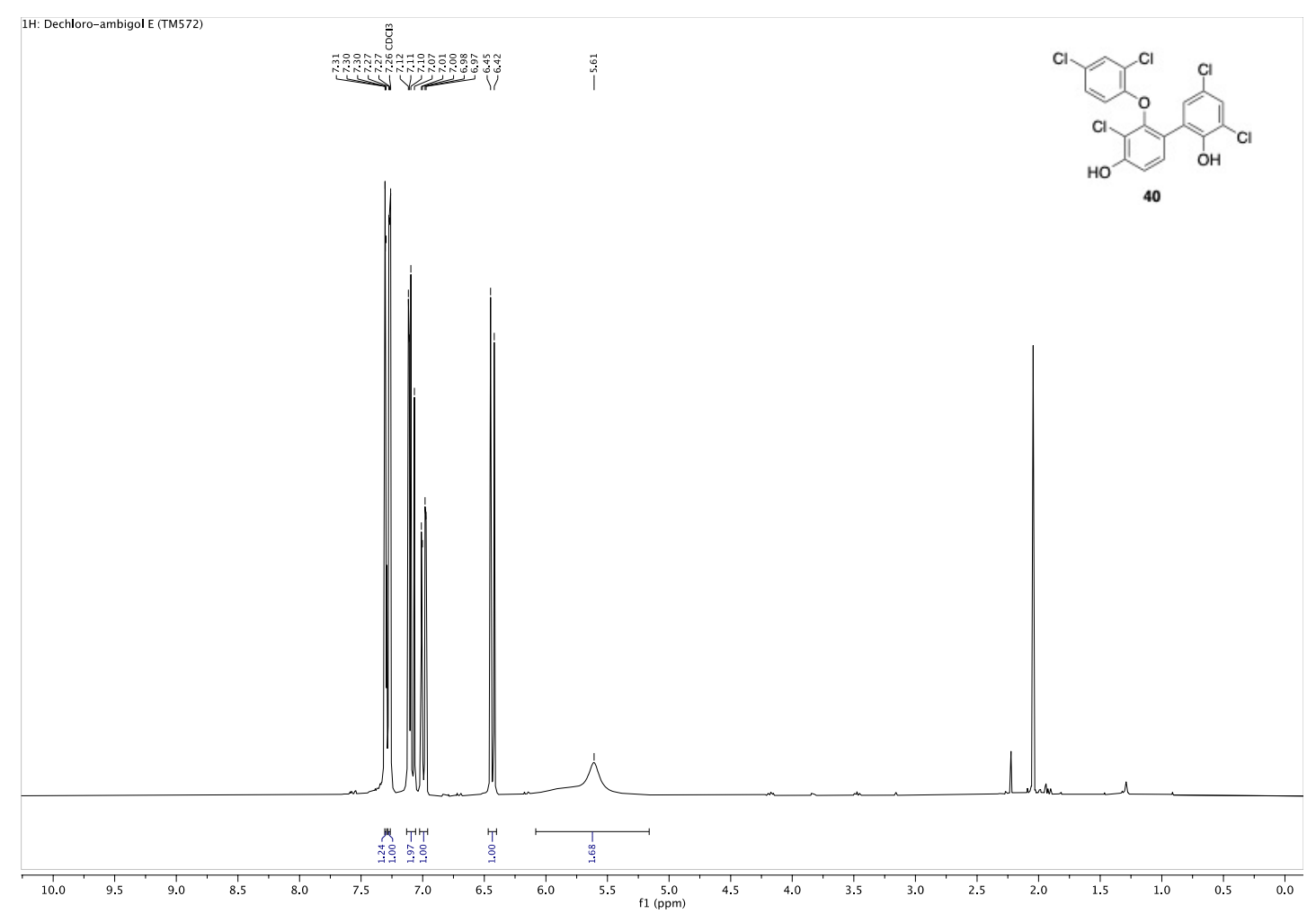

Fig. S70 ${ }^{1} \mathrm{H}-\mathrm{NMR}$ spectrum of 3,3',5-trichloro-2'-(2,4-dichlorophenoxy)-[1,1'-biphenyl]-2,4'-diol (40), measured in $\mathrm{CDCl}_{3}$ at $300 \mathrm{MHz}$.

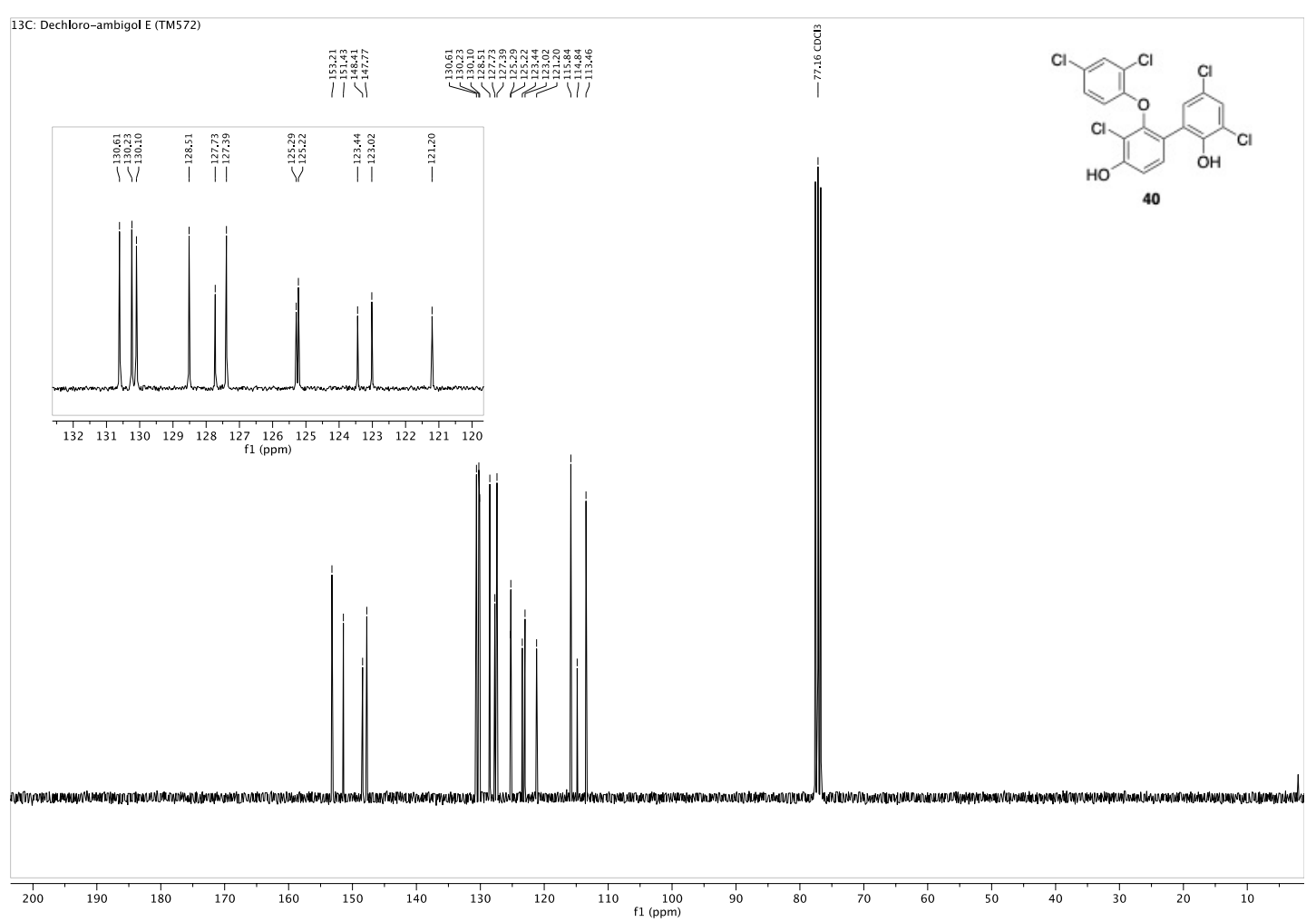

Fig. S71 ${ }^{13}$ C-NMR spectrum of 3,3',5-trichloro-2'-(2,4-dichlorophenoxy)-[1,1'-biphenyl]-2,4'-diol (40), measured in $\mathrm{CDCl}_{3}$ at $75 \mathrm{MHz}$. 

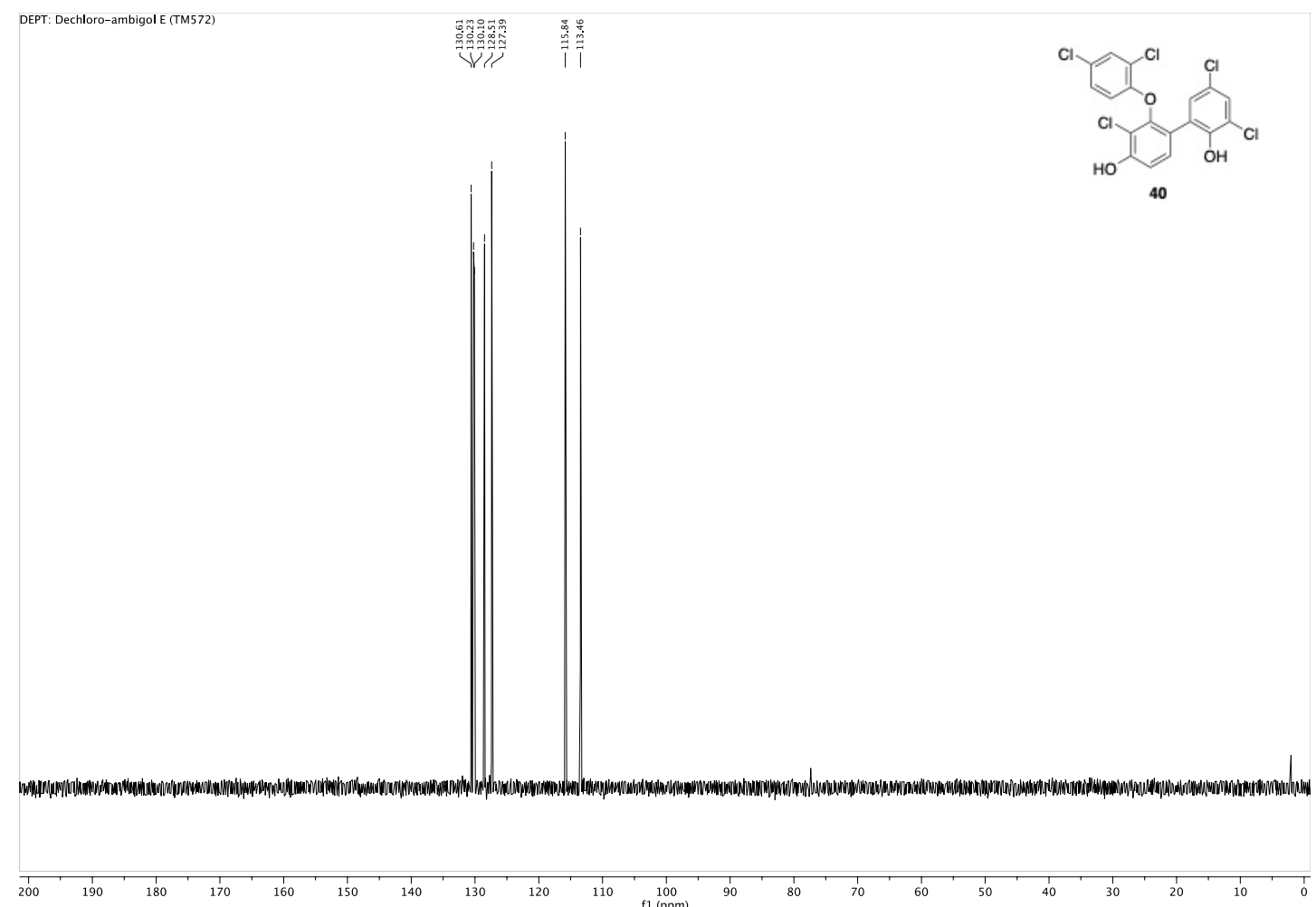

Fig. S72 DEPT spectrum of 3,3',5-trichloro-2'-(2,4-dichlorophenoxy)-[1,1'-biphenyl]-2,4'-diol (40), measured in $\mathrm{CDCl}_{3}$ at $75 \mathrm{MHz}$. 


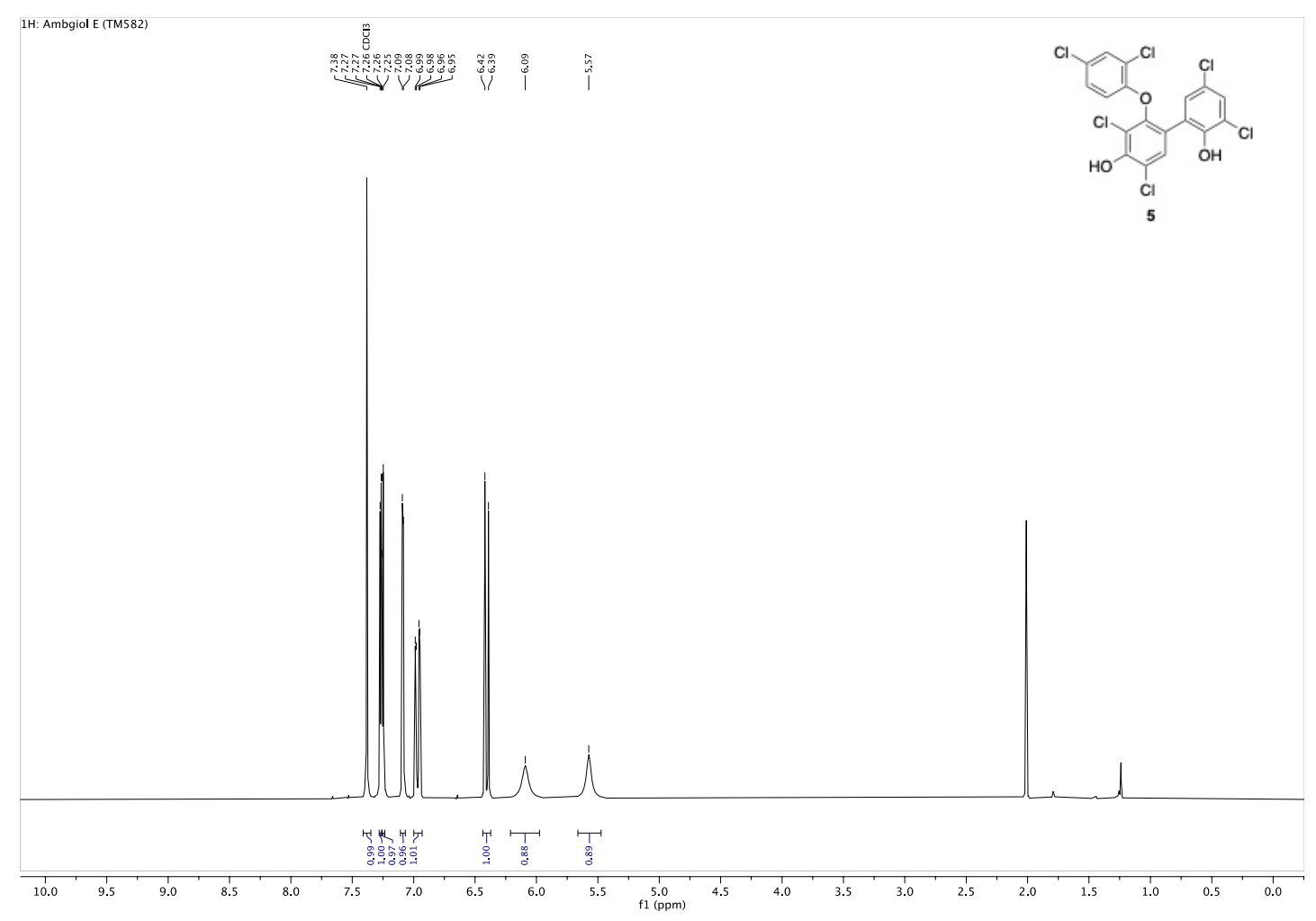

Fig. S73 ${ }^{1} \mathrm{H}-\mathrm{NMR}$ spectrum of ambigol E (5), measured in $\mathrm{CDCl}_{3}$ at $300 \mathrm{MHz}$.

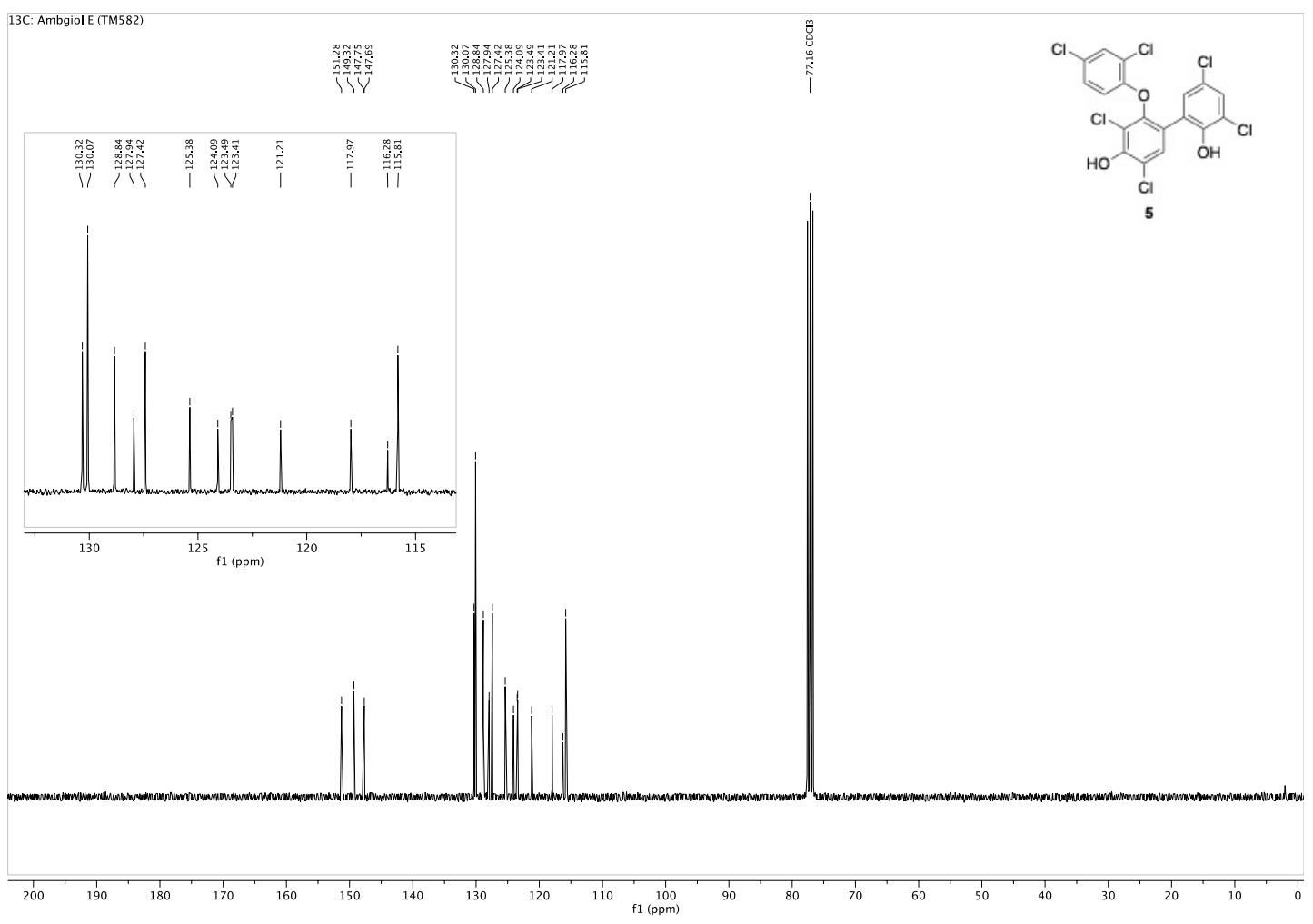

Fig. S74 ${ }^{13} \mathrm{C}-\mathrm{NMR}$ spectrum of ambigol E (5), measured in $\mathrm{CDCl}_{3}$ at $75 \mathrm{MHz}$. 


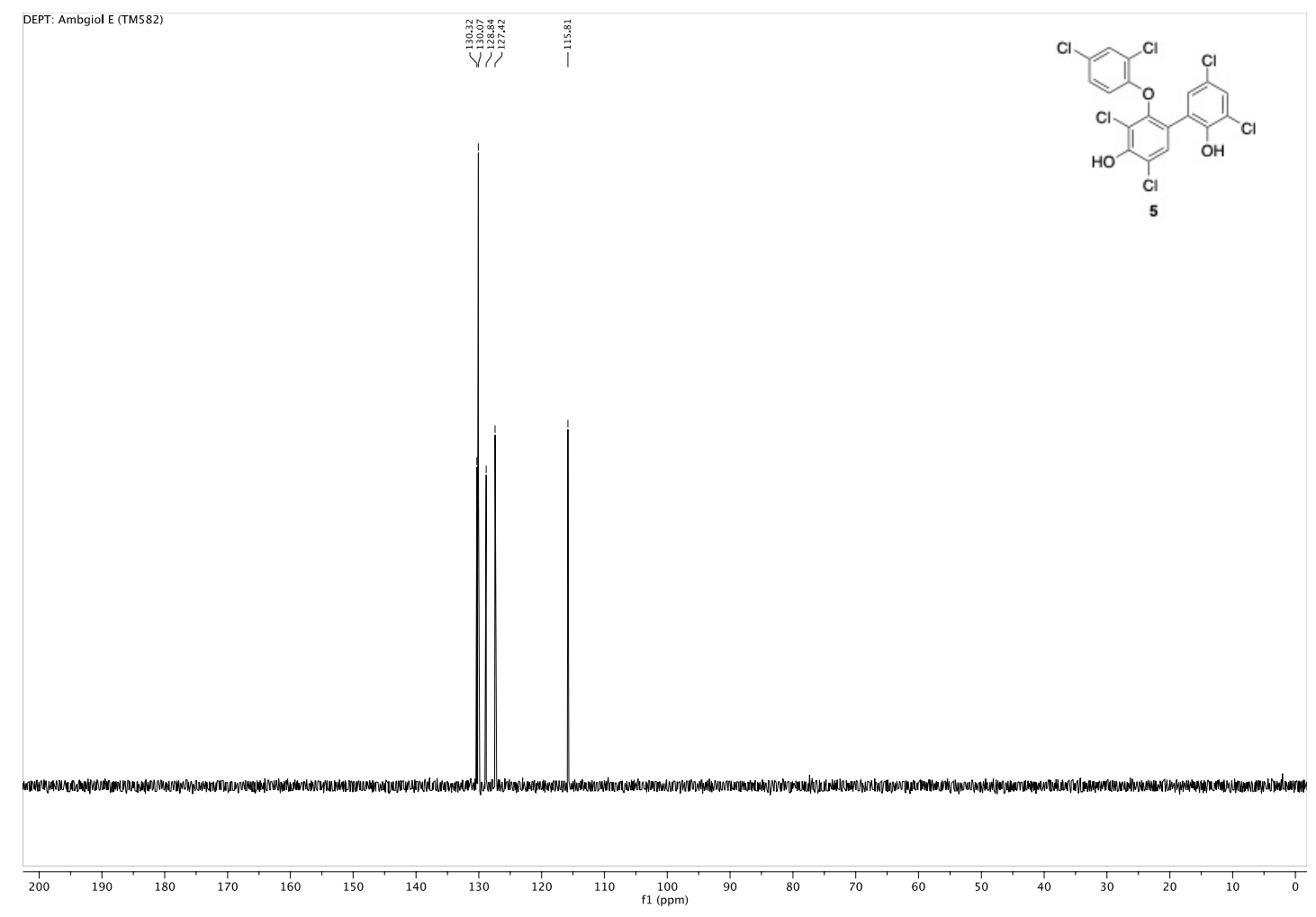

Fig. S75 DEPT spectrum of ambigol E (5), measured in $\mathrm{CDCl}_{3}$ at $75 \mathrm{MHz}$.

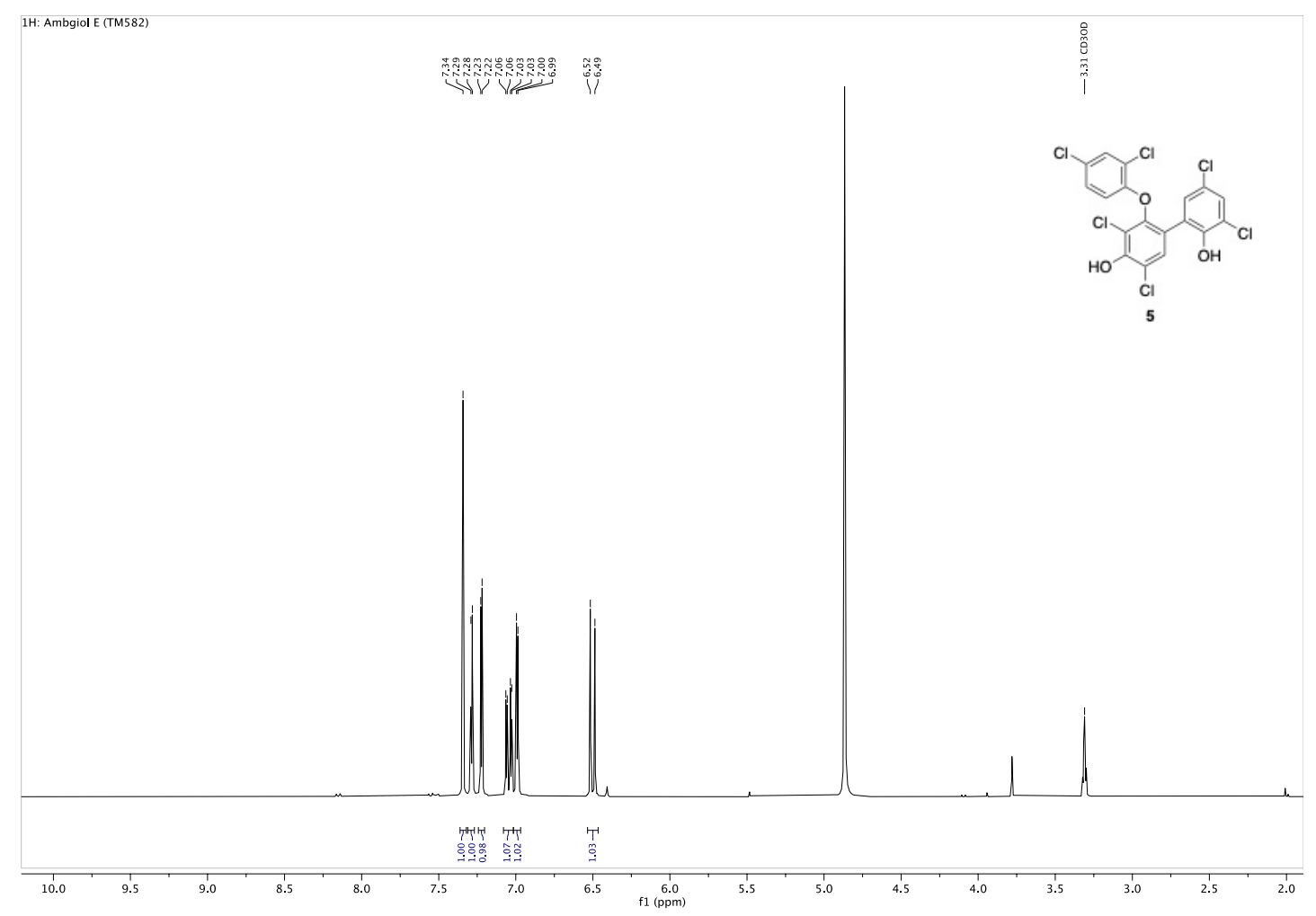

Fig. S76 ${ }^{1} \mathrm{H}-\mathrm{NMR}$ spectrum of ambigol E (5), measured in MeOD-d 4 at $300 \mathrm{MHz}$. 


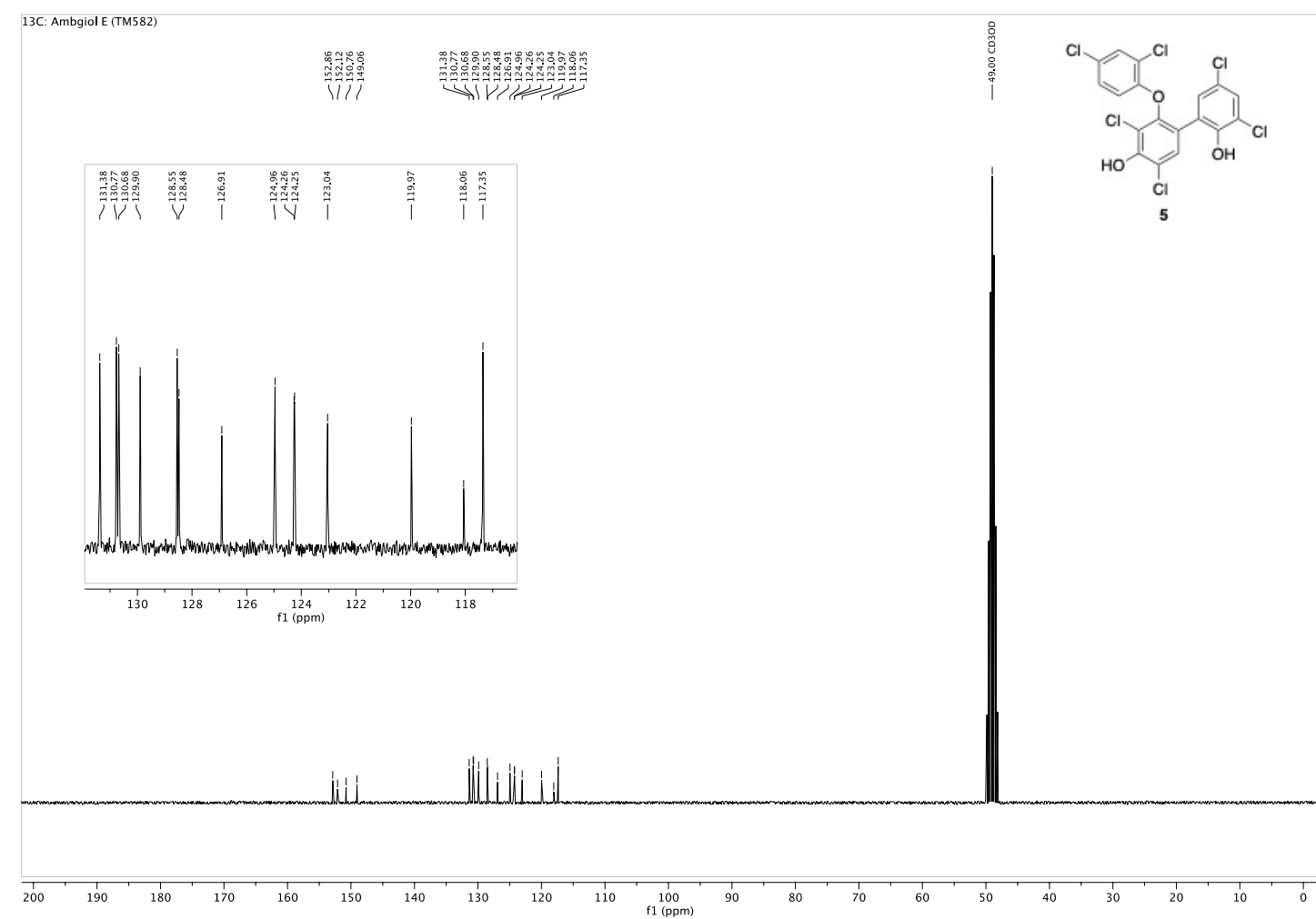

Fig. S77 ${ }^{13} \mathrm{C}-\mathrm{NMR}$ spectrum of ambigol E (5), measured in MeOD-d 4 at $75 \mathrm{MHz}$.
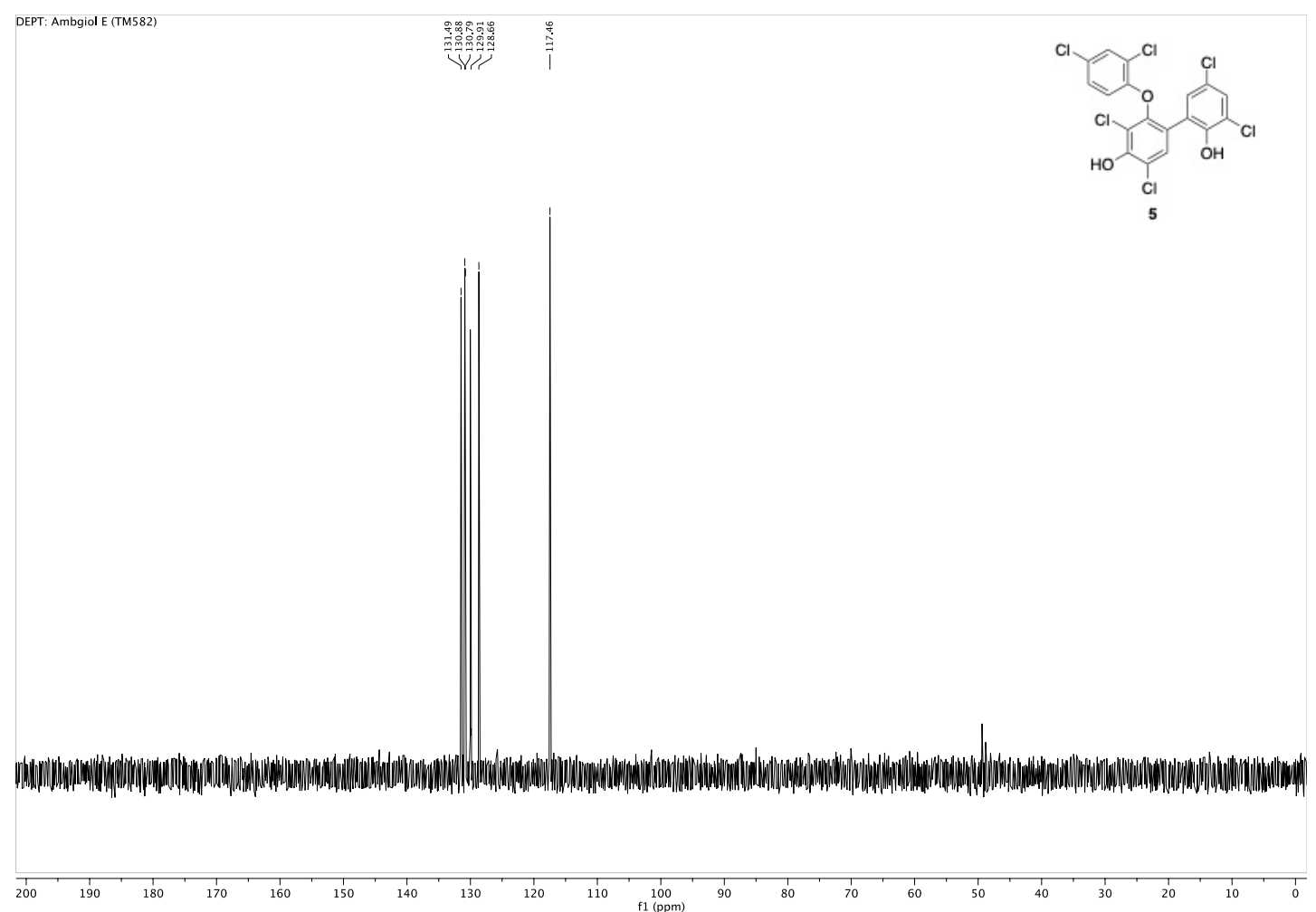

Fig. S78 DEPT spectrum of ambigol E (5), measured in MeOD- $\mathrm{d}_{4}$ at $75 \mathrm{MHz}$. 


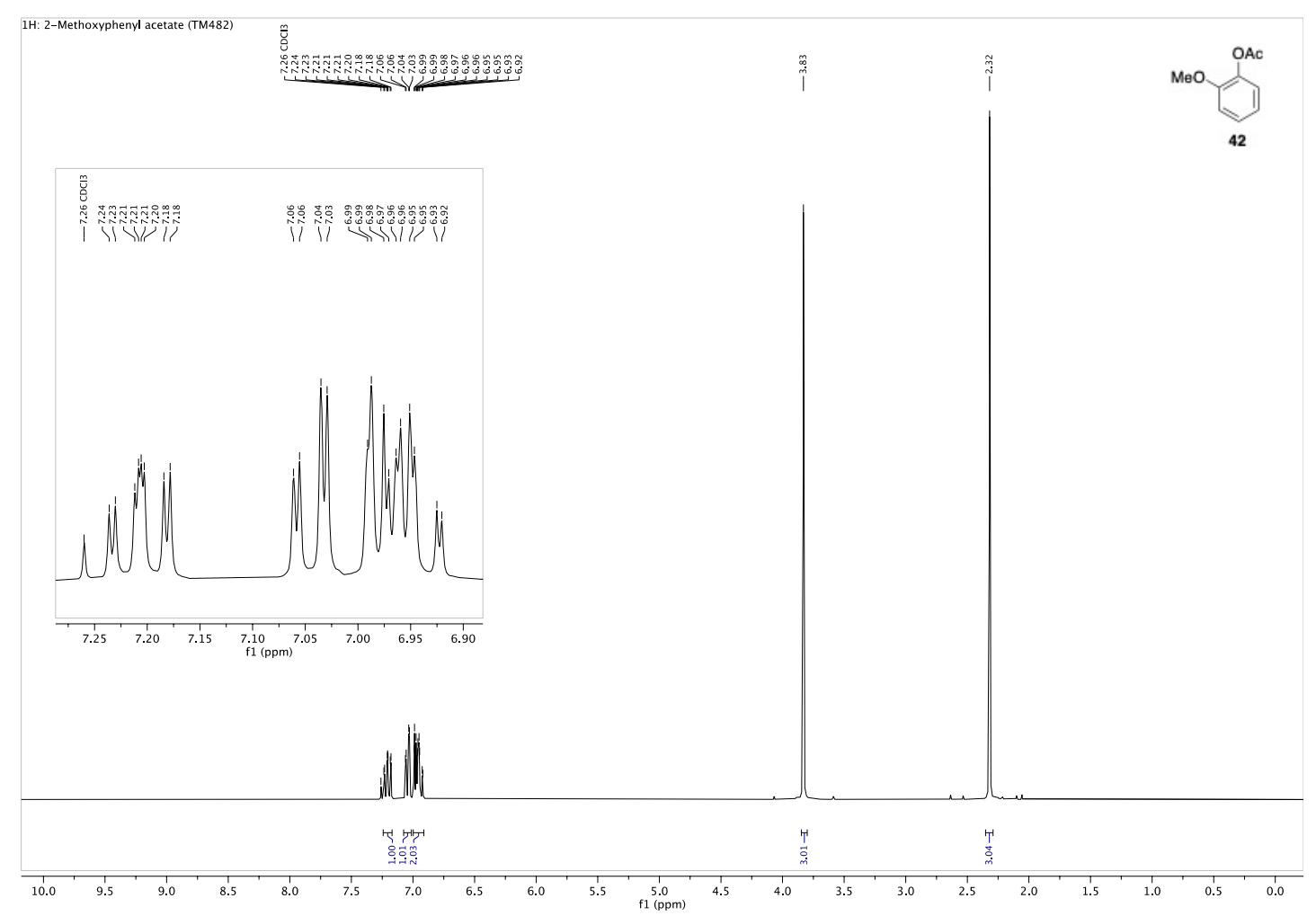

Fig. S79 ${ }^{1} \mathrm{H}$-NMR spectrum of 2-methoxyphenyl acetate (42), measured in $\mathrm{CDCl}_{3}$ at $300 \mathrm{MHz}$.
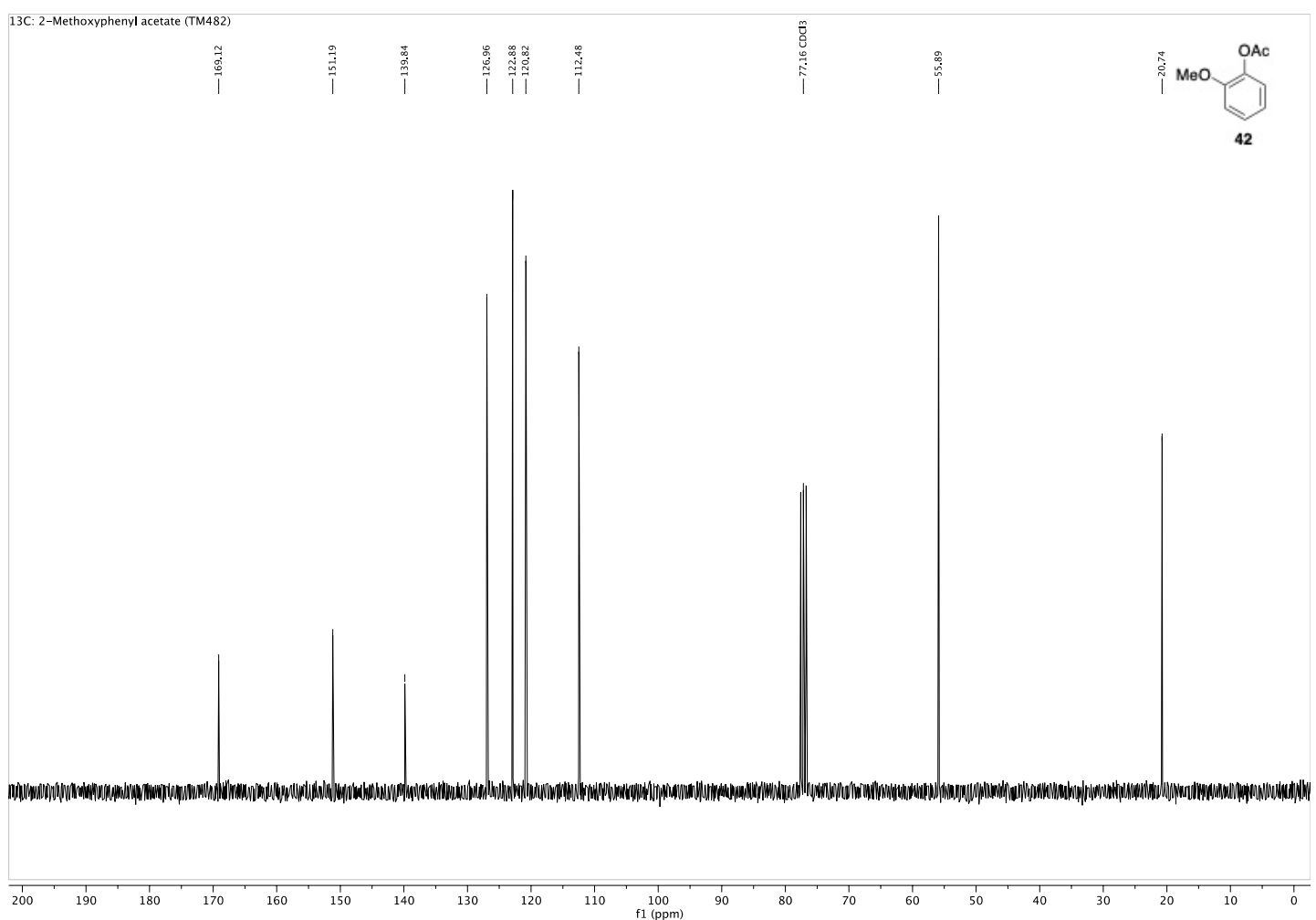

Fig. S80 ${ }^{13} \mathrm{C}$-NMR spectrum of 2-methoxyphenyl acetate (42), measured in $\mathrm{CDCl}_{3}$ at $75 \mathrm{MHz}$. 

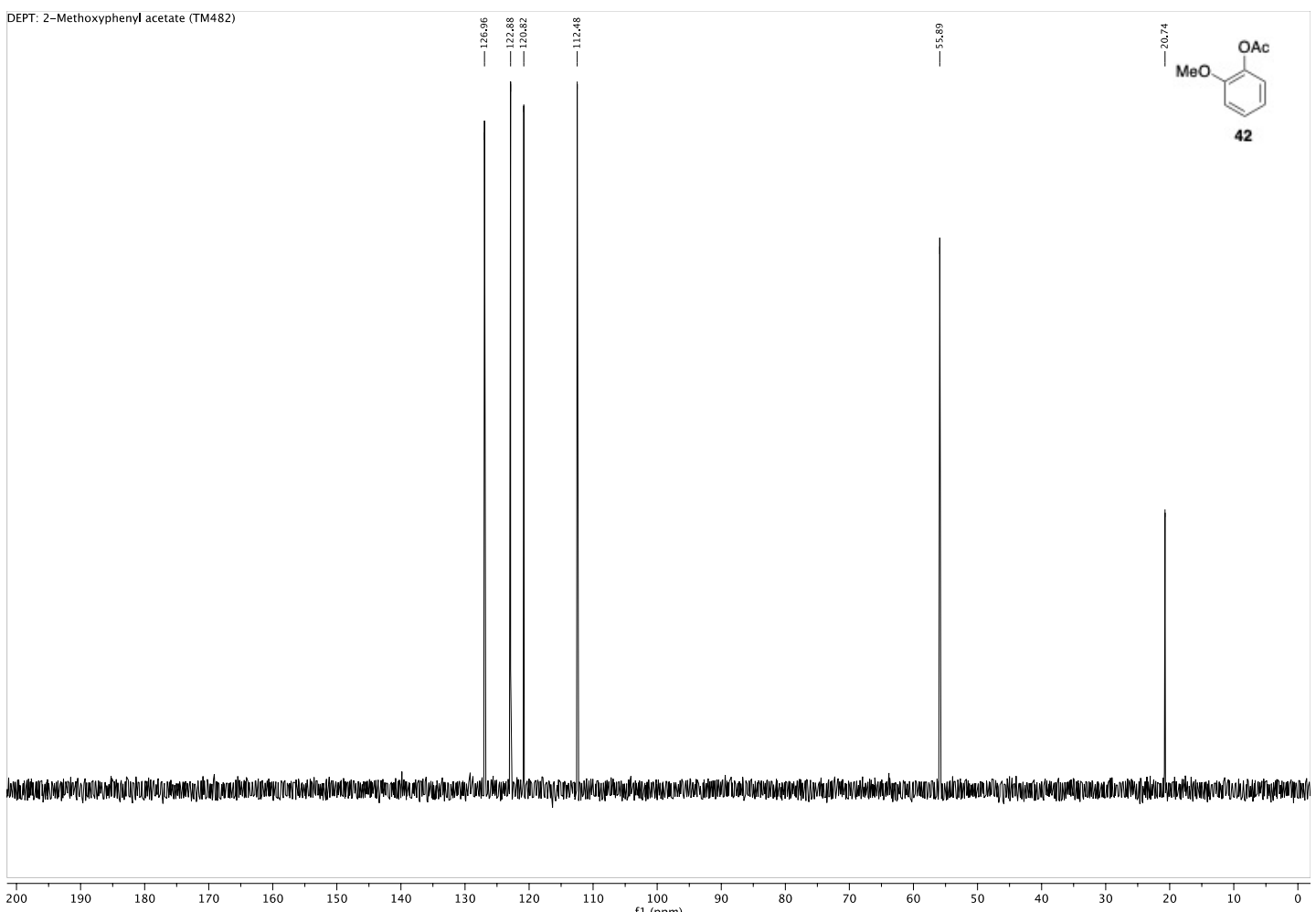

Fig. S81 DEPT spectrum of 2-methoxyphenyl acetate (42), measured in $\mathrm{CDCl}_{3}$ at $75 \mathrm{MHz}$. 


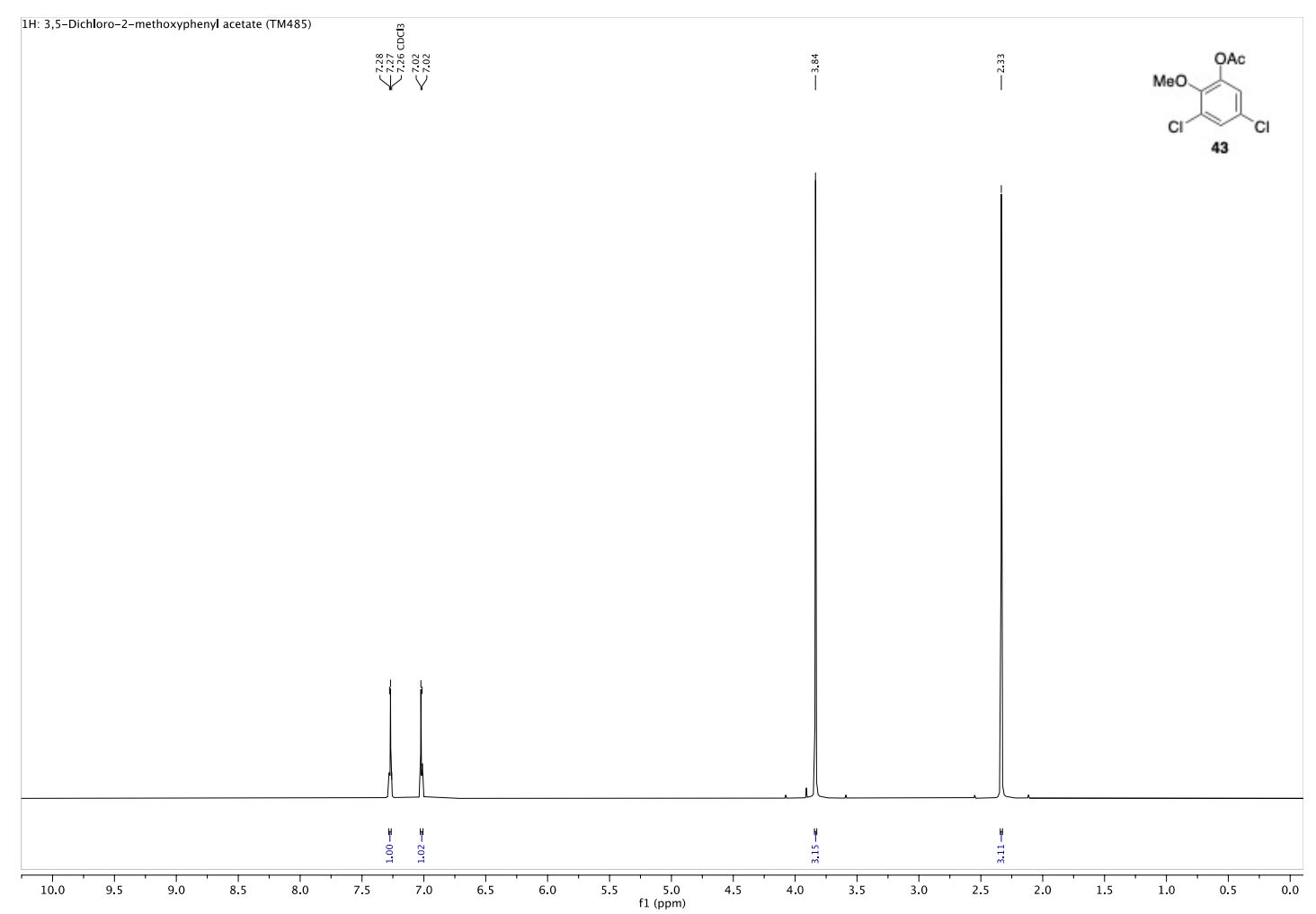

Fig. S82 ${ }^{1} \mathrm{H}-\mathrm{NMR}$ spectrum of 3,5-dichloro-2-methoxyphenyl acetate (43), measured in $\mathrm{CDCl}_{3}$ at $300 \mathrm{MHz}$.

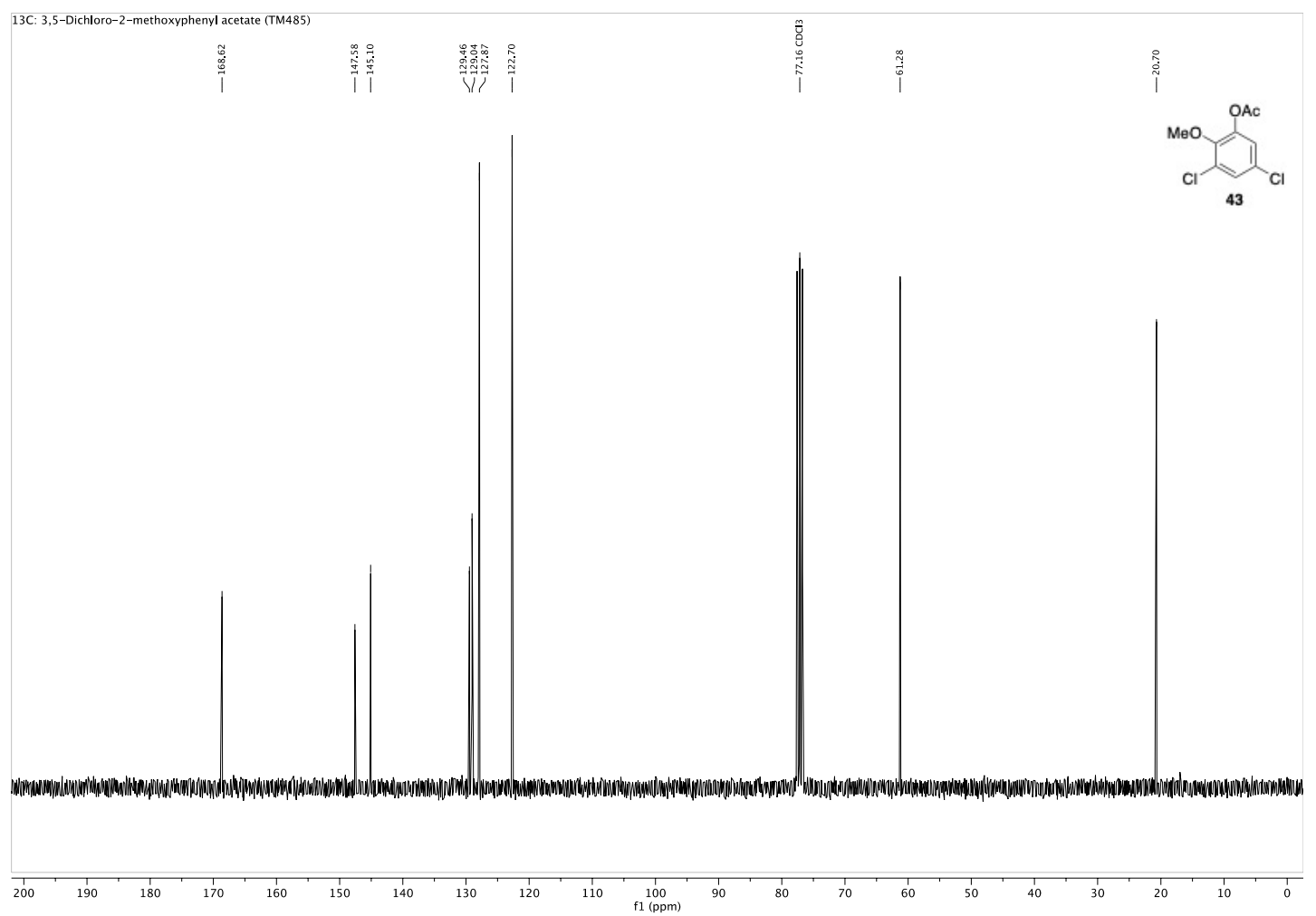

Fig. S83 ${ }^{13} \mathrm{C}-\mathrm{NMR}$ spectrum of 3,5-dichloro-2-methoxyphenyl acetate (43), measured in $\mathrm{CDCl}_{3}$ at $75 \mathrm{MHz}$. 


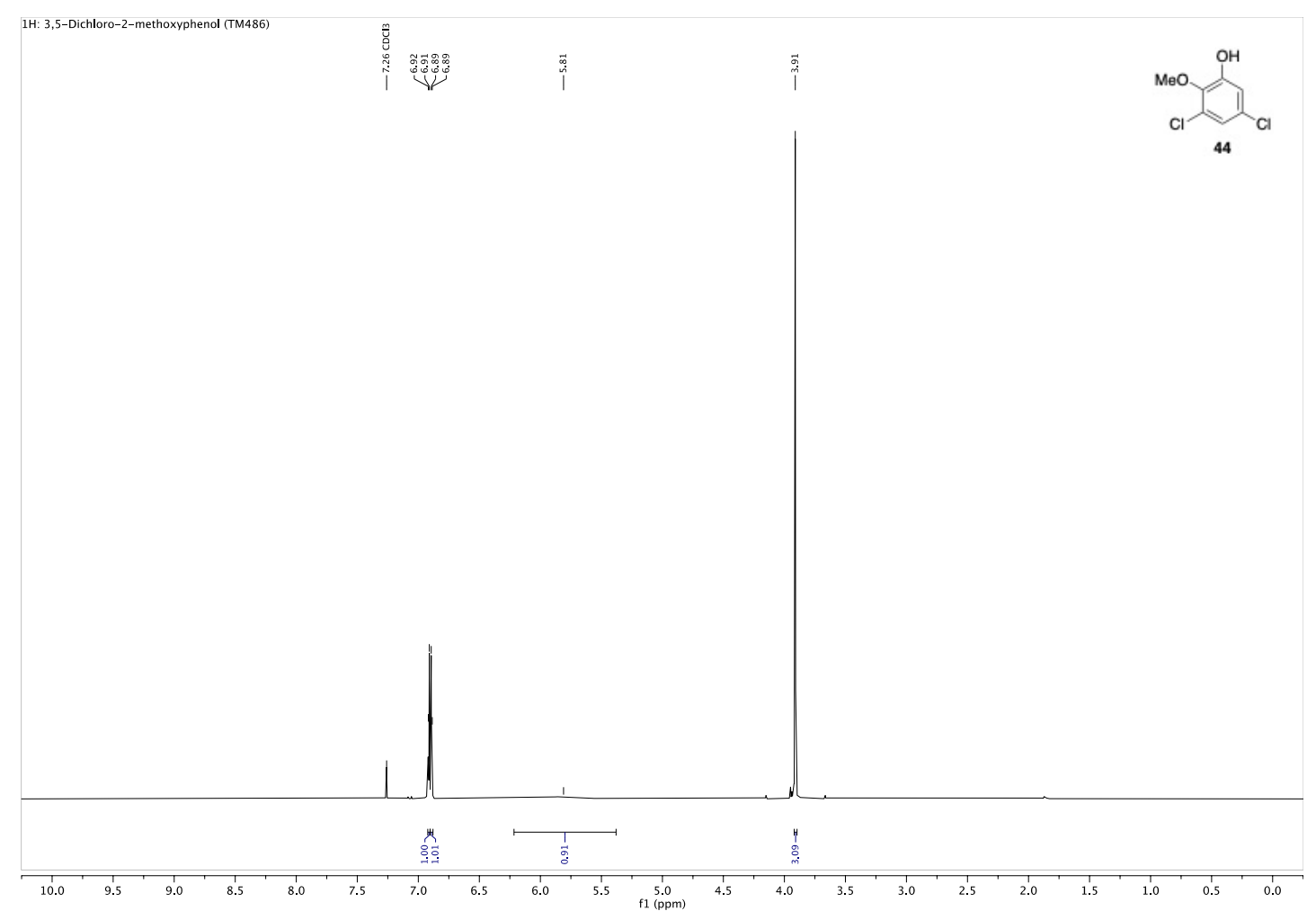

Fig. S84 ${ }^{1} \mathrm{H}-\mathrm{NMR}$ spectrum of 3,5-dichloro-2-methoxyphenol (44), measured in $\mathrm{CDCl}_{3}$ at $300 \mathrm{MHz}$.

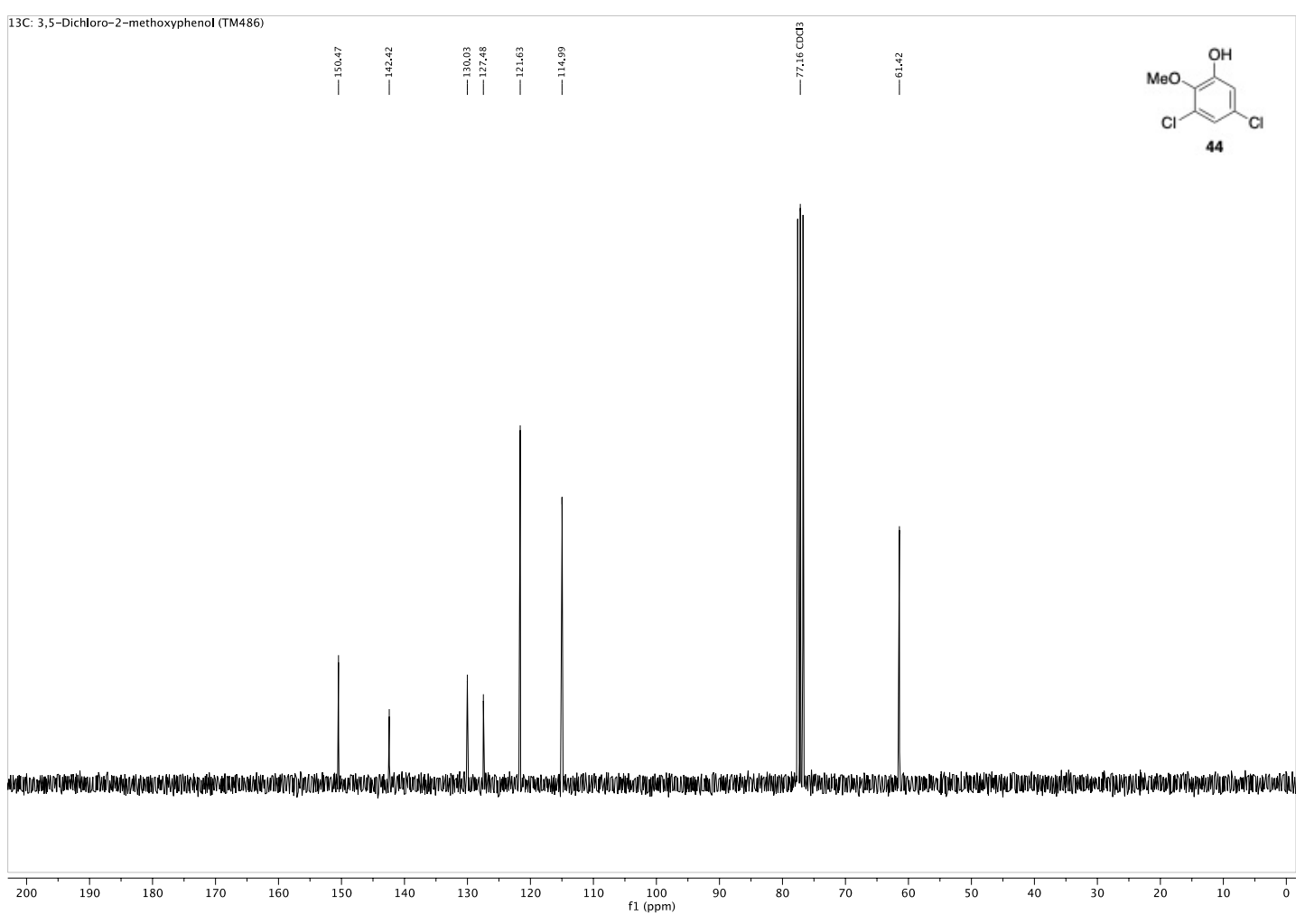

Fig. S85 ${ }^{13} \mathrm{C}-\mathrm{NMR}$ spectrum of 3,5-dichloro-2-methoxyphenol (44), measured in $\mathrm{CDCl}_{3}$ at $75 \mathrm{MHz}$. 

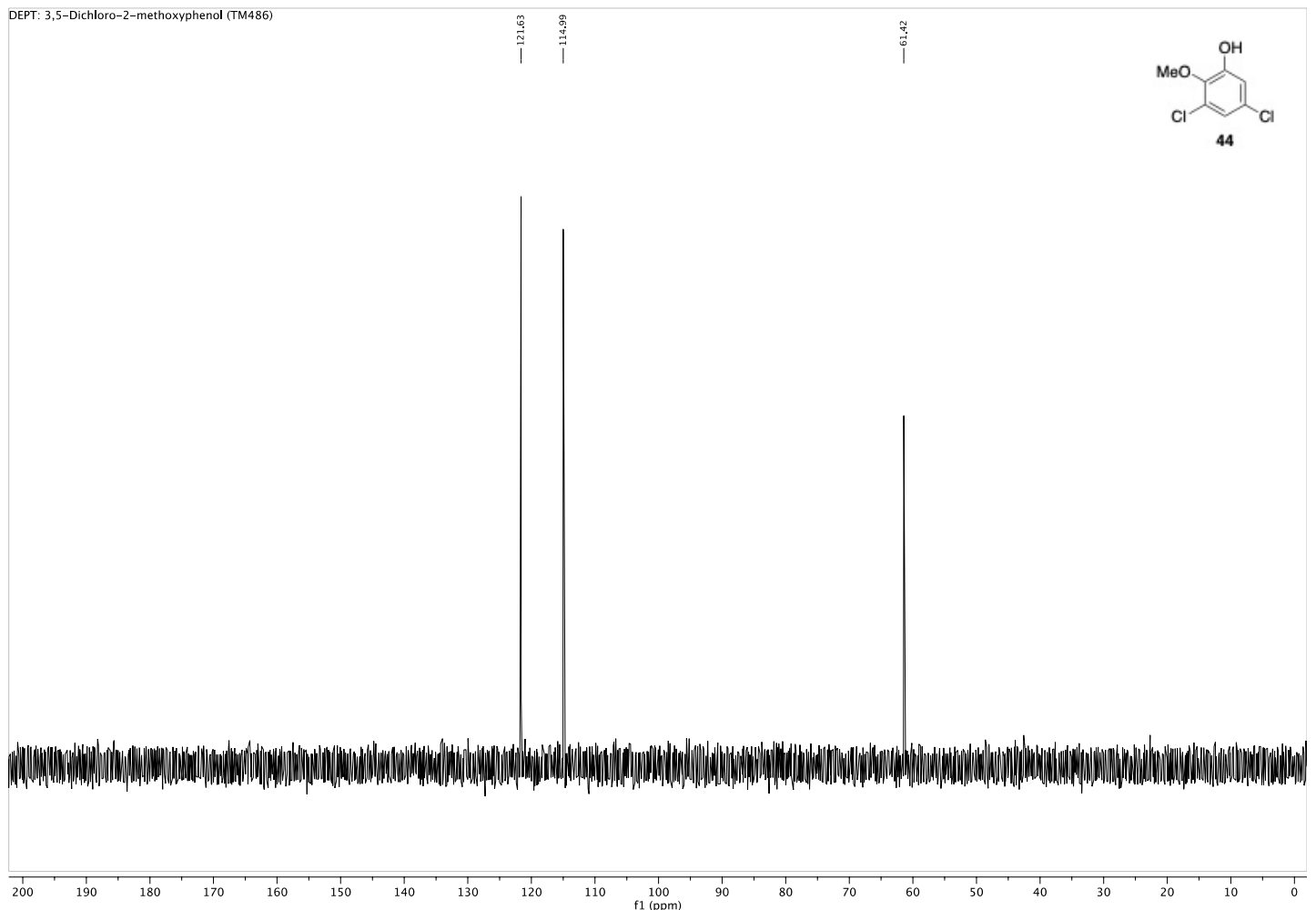

Fig. S86 DEPT spectrum of 3,5-dichloro-2-methoxyphenol (44), measured in $\mathrm{CDCl}_{3}$ at $75 \mathrm{MHz}$. 


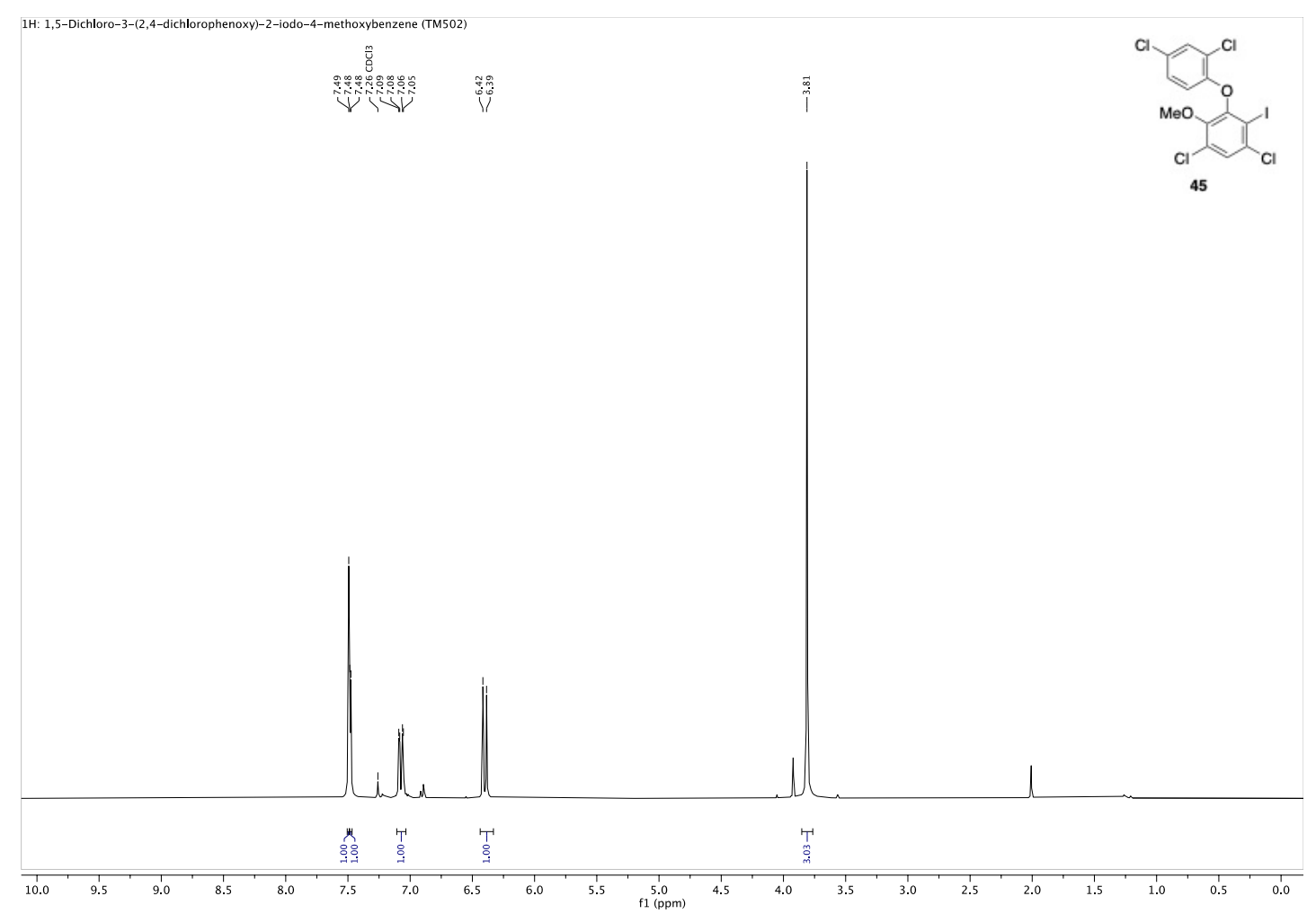

Fig. S87 ${ }^{1} \mathrm{H}-\mathrm{NMR}$ spectrum of 1,5-dichloro-3-(2,4-dichlorophenoxy)-2-iodo-4-methoxybenzene (45), measured in $\mathrm{CDCl}_{3}$ at $300 \mathrm{MHz}$.

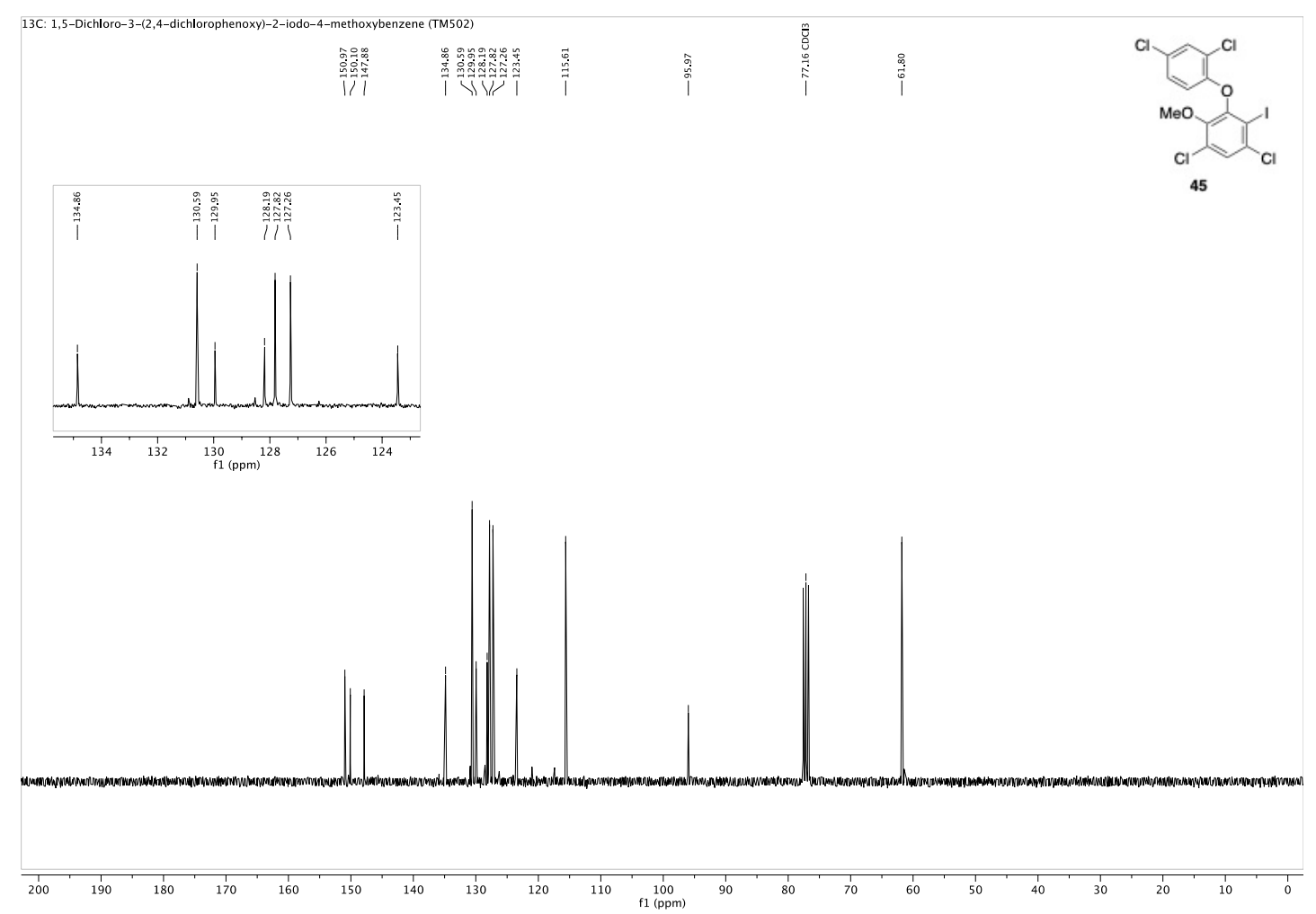

Fig. S88 ${ }^{13} \mathrm{C}$-NMR spectrum of 1,5-dichloro-3-(2,4-dichlorophenoxy)-2-iodo-4-methoxybenzene (45), measured in $\mathrm{CDCl}_{3}$ at $75 \mathrm{MHz}$. 


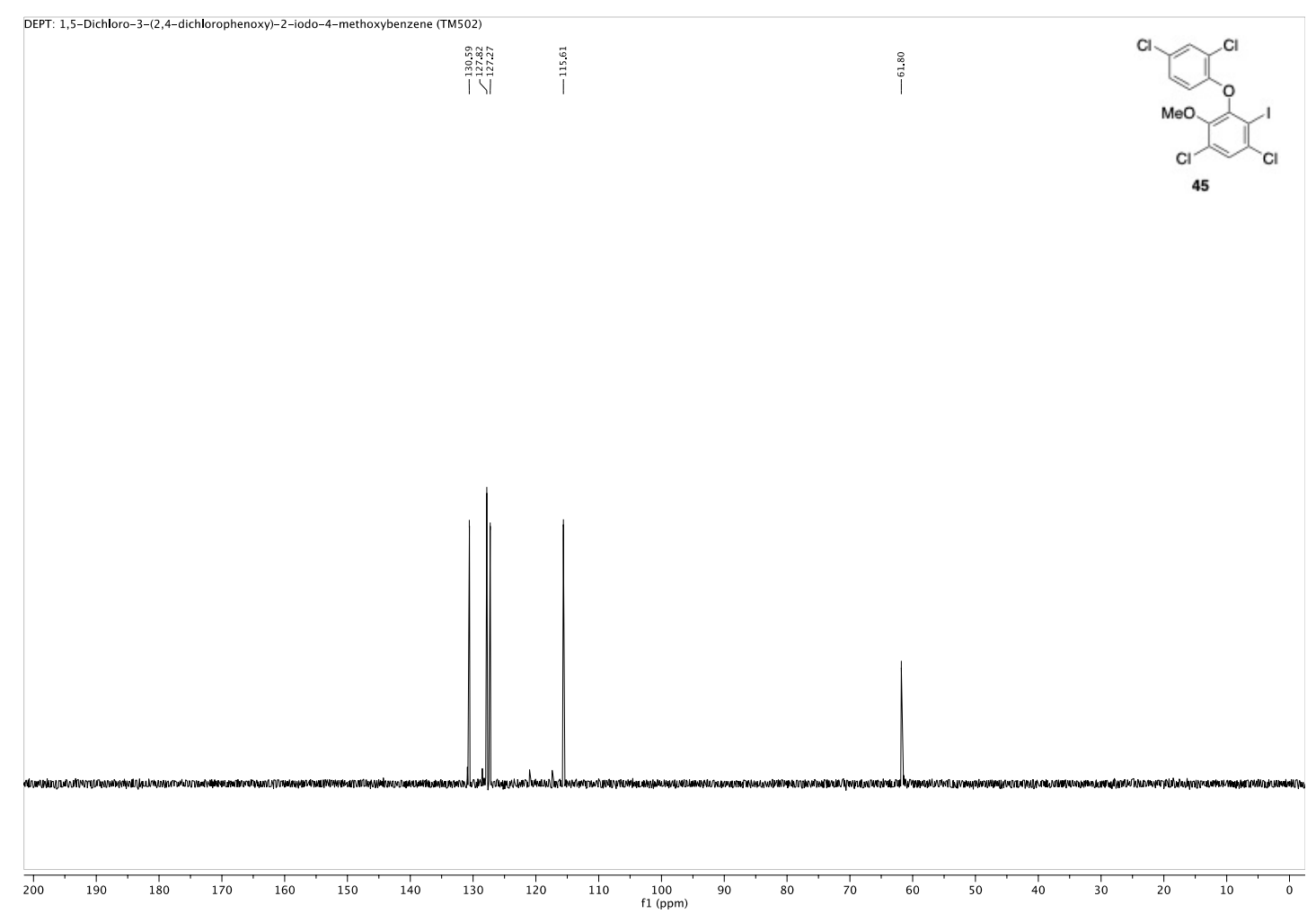

Fig. S89 DEPT spectrum of 1,5-dichloro-3-(2,4-dichlorophenoxy)-2-iodo-4-methoxybenzene (45), measured in $\mathrm{CDCl}_{3}$ at $75 \mathrm{MHz}$. 


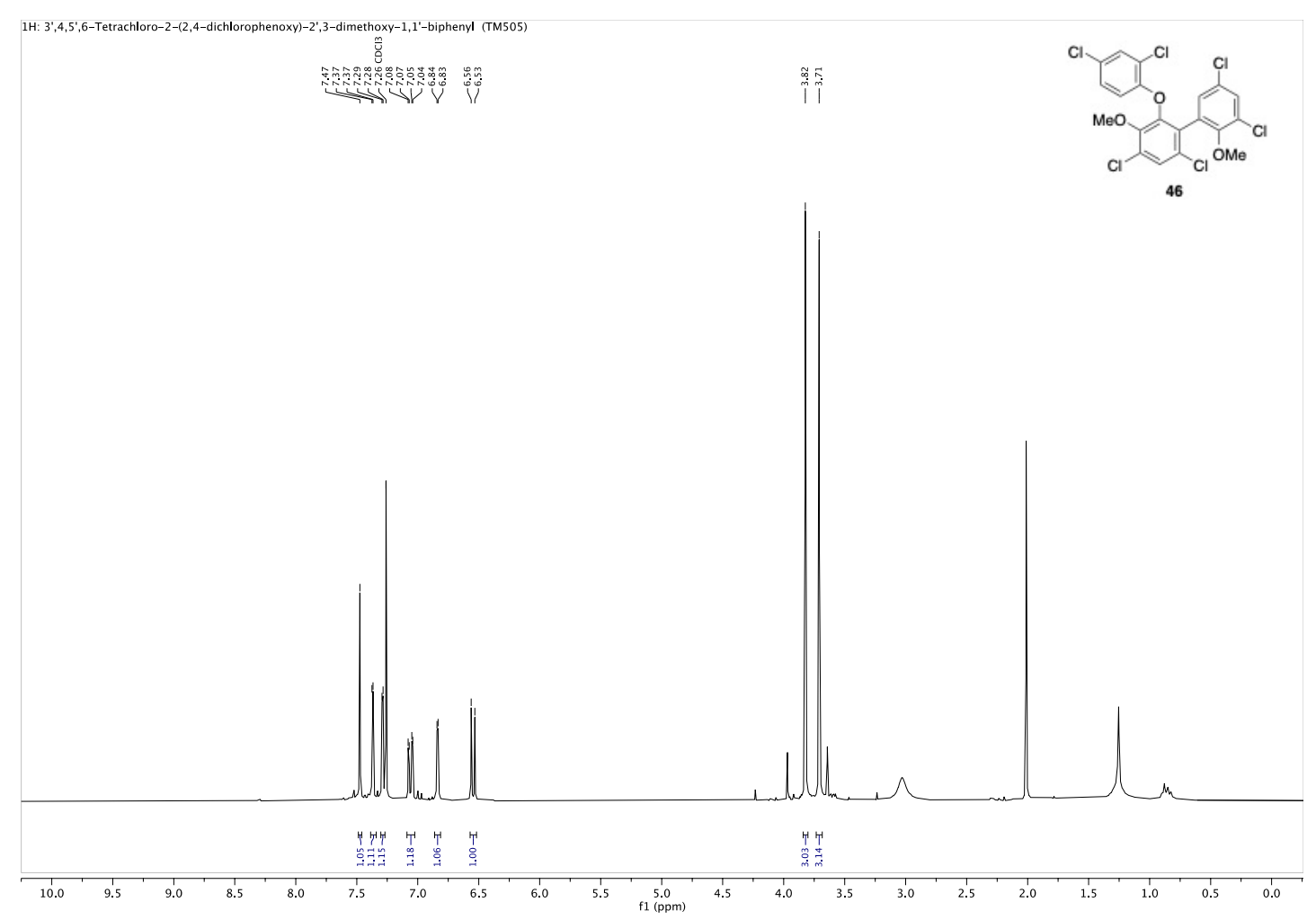

Fig. S90 ${ }^{1} \mathrm{H}-\mathrm{NMR}$ spectrum of 3',4,5',6-tetrachloro-2-(2,4-dichlorophenoxy)-2',3-dimethoxy-1,1'-biphenyl (46), measured in $\mathrm{CDCl}_{3}$ at $300 \mathrm{MHz}$.

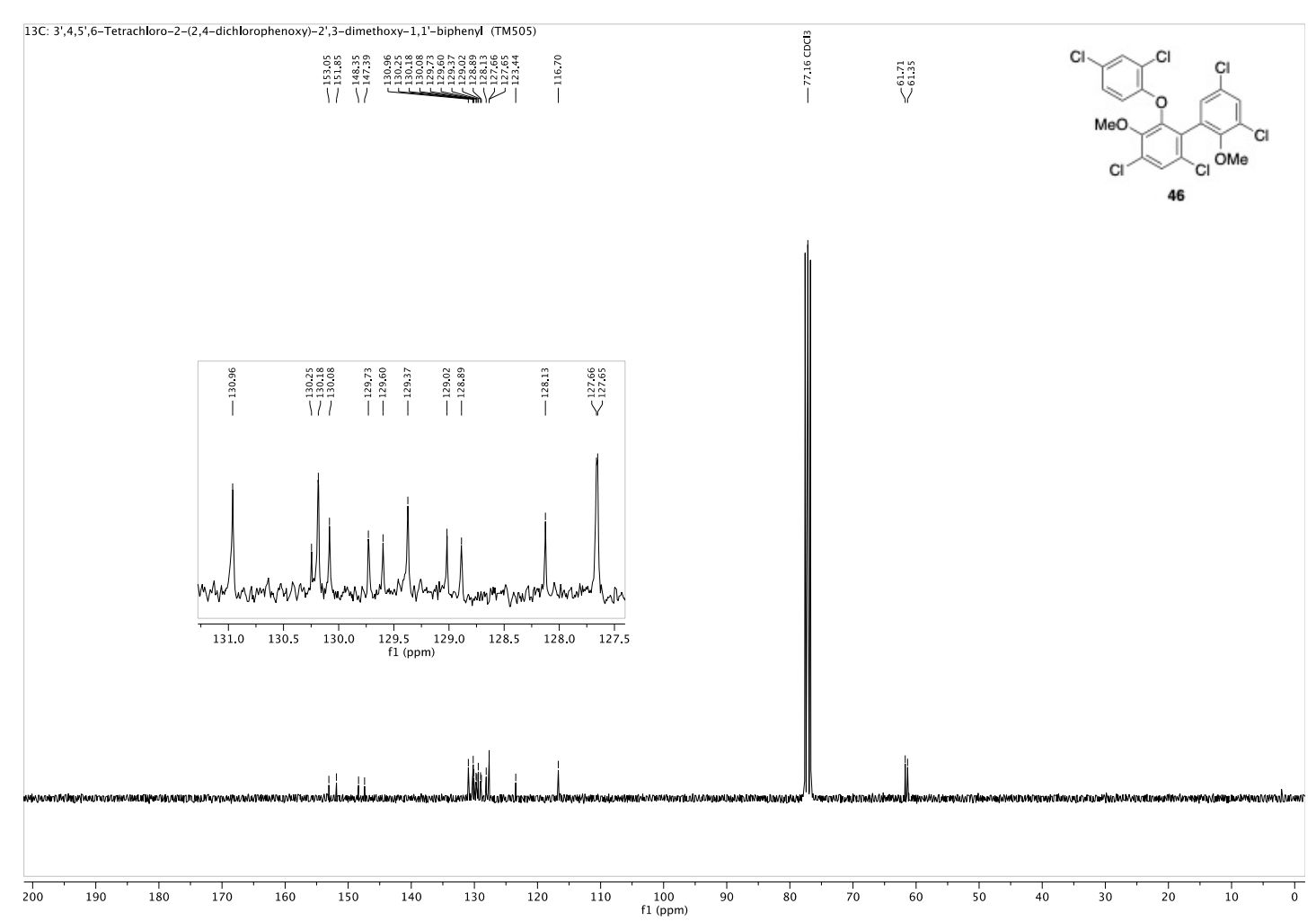

Fig. S91 ${ }^{13} \mathrm{C}$-NMR spectrum of 3',4,5',6-tetrachloro-2-(2,4-dichlorophenoxy)-2',3-dimethoxy-1,1'-biphenyl (46), measured in $\mathrm{CDCl}_{3}$ at $75 \mathrm{MHz}$. 


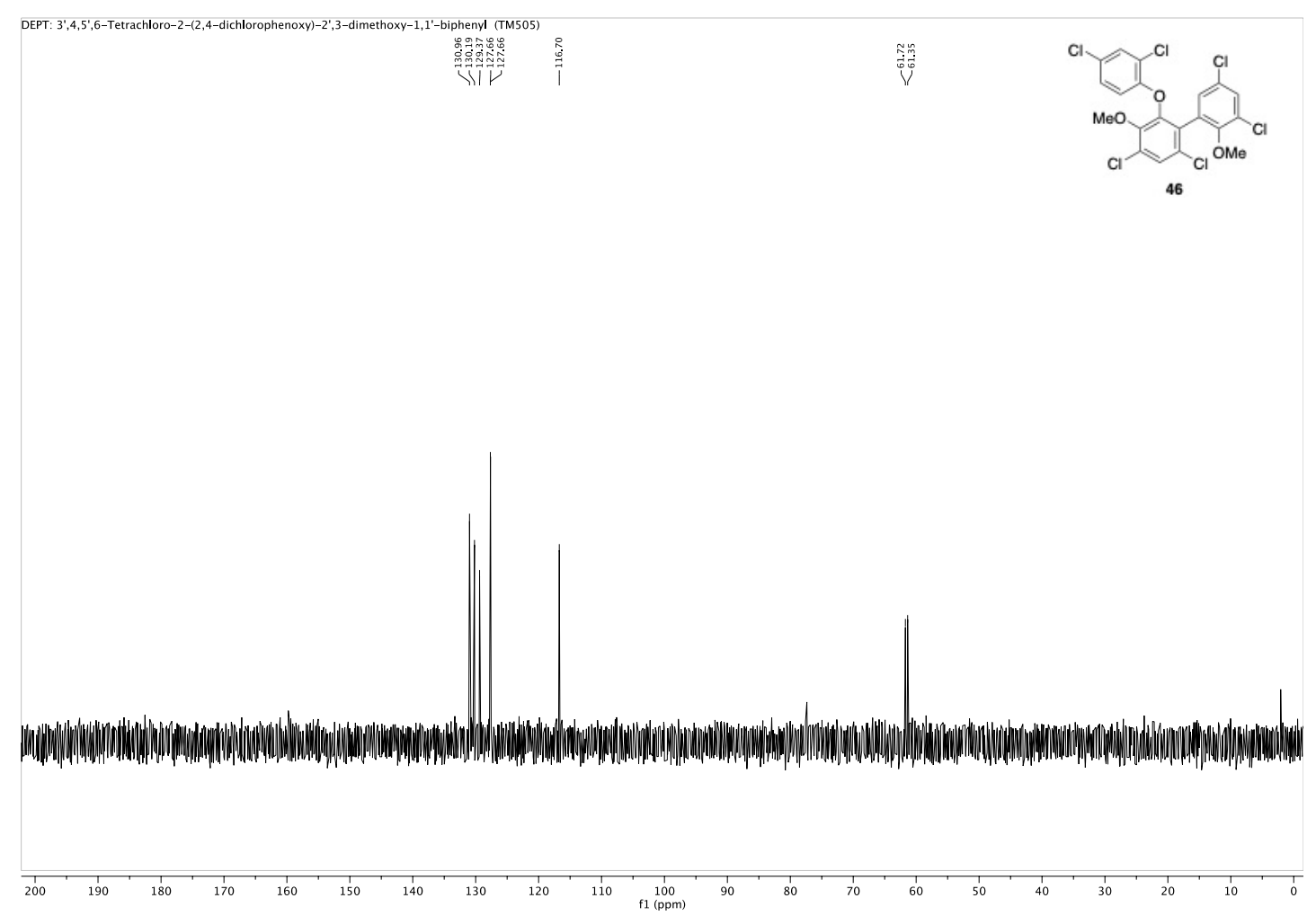

Fig. S92 DEPT spectrum of 3',4,5',6-tetrachloro-2-(2,4-dichlorophenoxy)-2',3-dimethoxy-1,1'-biphenyl (46), measured in $\mathrm{CDCl}_{3}$ at $75 \mathrm{MHz}$. 


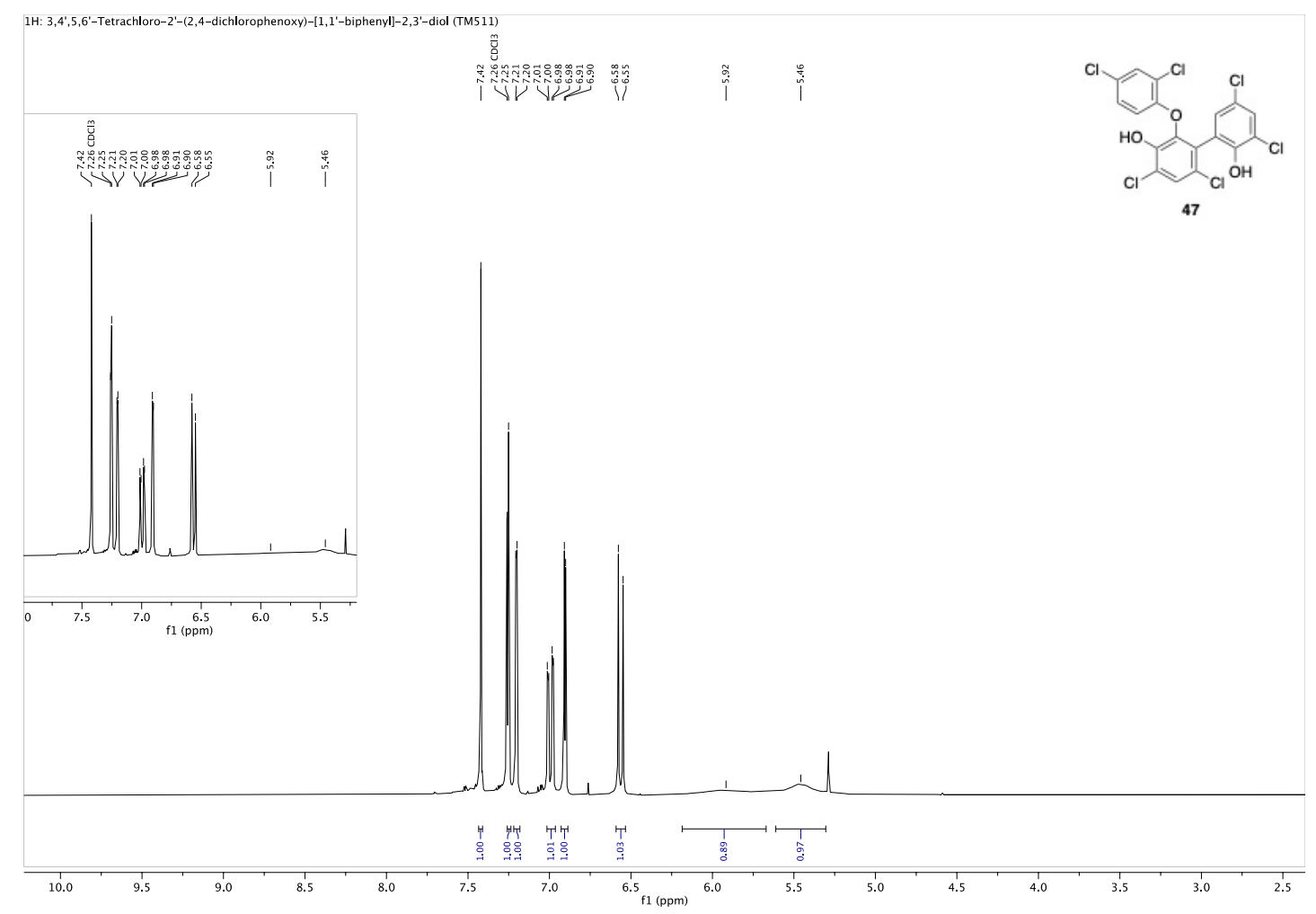

Fig. S93 ${ }^{1} \mathrm{H}$-NMR spectrum of 3,4',5,6'-tetrachloro-2'-(2,4-dichlorophenoxy)-[1,1'-biphenyl]-2,3'-diol (47), measured in $\mathrm{CDCl}_{3}$ at $300 \mathrm{MHz}$.

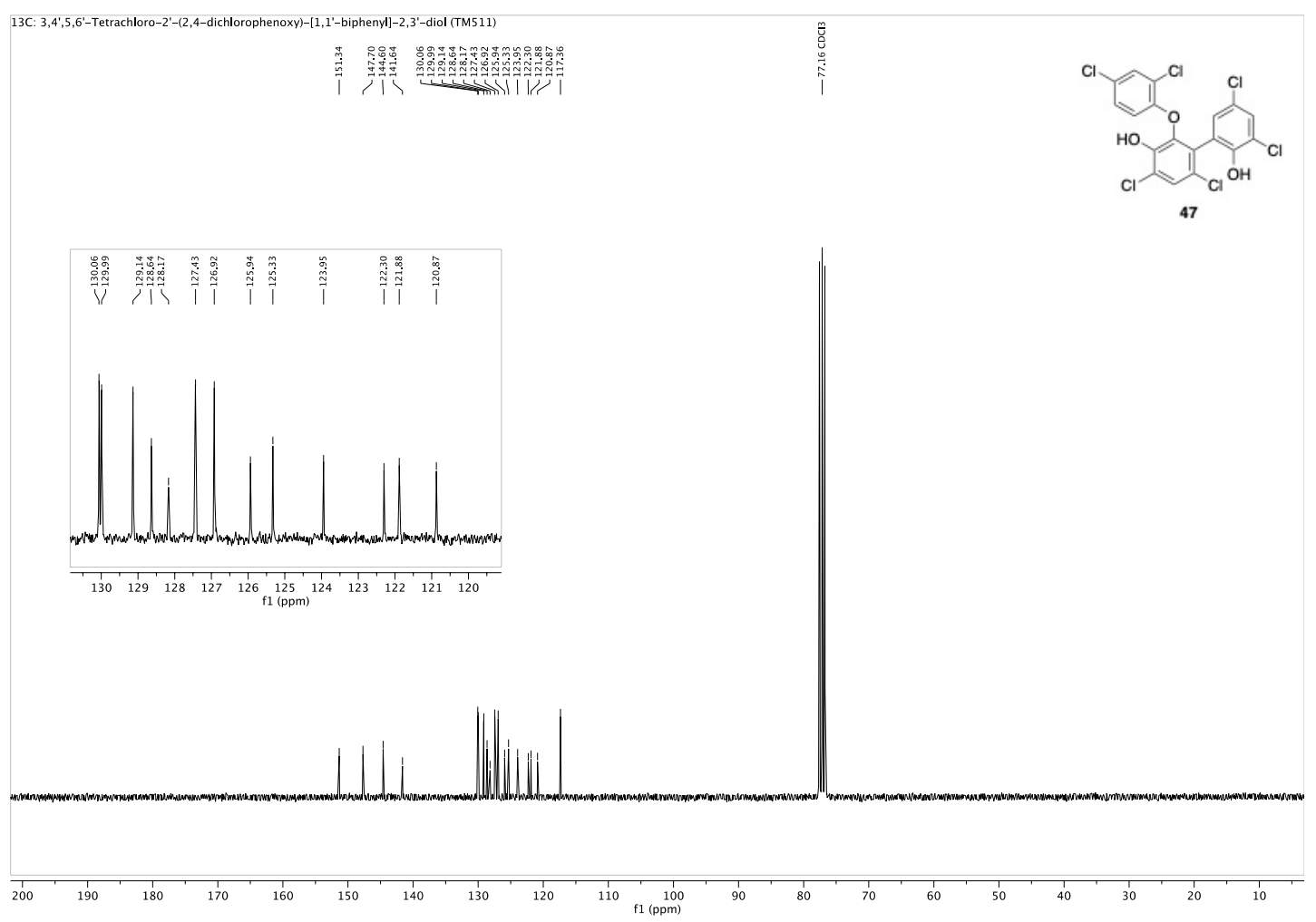

Fig. $S 94{ }^{13}$ C-NMR spectrum of 3,4',5,6'-tetrachloro-2'-(2,4-dichlorophenoxy)-[1,1'-biphenyl]-2,3'-diol (47), measured in $\mathrm{CDCl}_{3}$ at $75 \mathrm{MHz}$. 

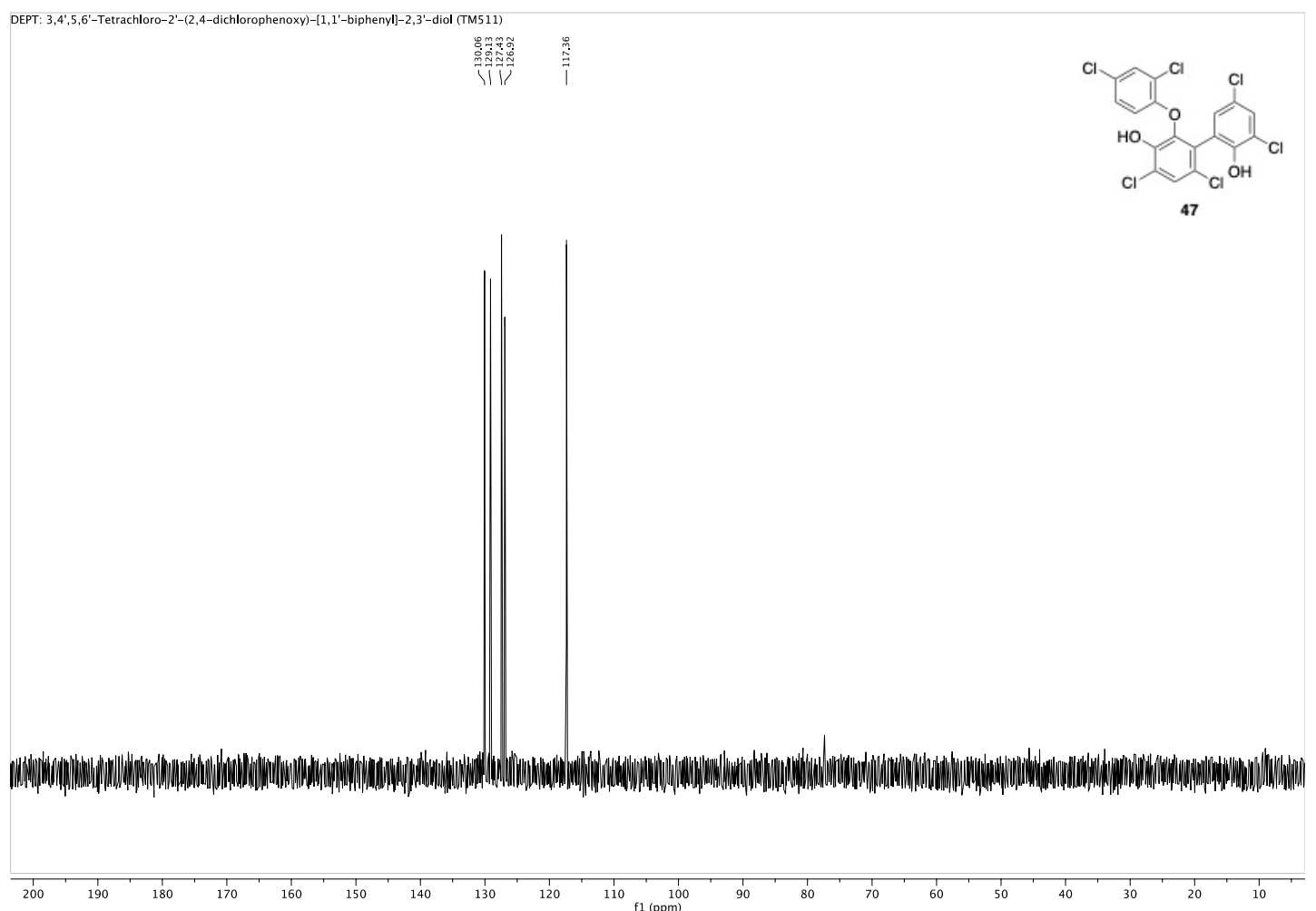

Fig. S95 DEPT spectrum of 3,4',5,6'-tetrachloro-2'-(2,4-dichlorophenoxy)-[1,1'-biphenyl]-2,3'-diol (47), measured in $\mathrm{CDCl}_{3}$ at $75 \mathrm{MHz}$. 


\section{Analysis of Rotational Stability of Ambigol A (1)}

HPLC analysis was performed on a computer-controlled Jasco system including a UV-1575 Intelligent UV/VIS Detector, DG-2080-53 3-Line Degaser, two PU-1580 Intelligent HPLC Pumps, AS-1550 Intelligent Sampler, HG-1580-32 Dynamic Mixer. A NUCLEOCEL DELTA-RP S, $5 \mu \mathrm{m}$ $(250 \times 4.6 \mathrm{~mm})$ chiral column manufactured by Machinery-Nagel was used as the stationary phase with the eluent system $A=\mathrm{H}_{2} \mathrm{O}+0.05 \%$ TFA, $B=\mathrm{MeCN}+0.05 \%$ TFA. Separation was achieved under isocratic conditions $(62 \% \mathrm{~B}, 60 \mathrm{~min})$ with a flow rate of $0.7 \mathrm{~mL} / \mathrm{min}$ at $5{ }^{\circ} \mathrm{C}$. All UV traces are shown at $220 \mathrm{~nm}$.

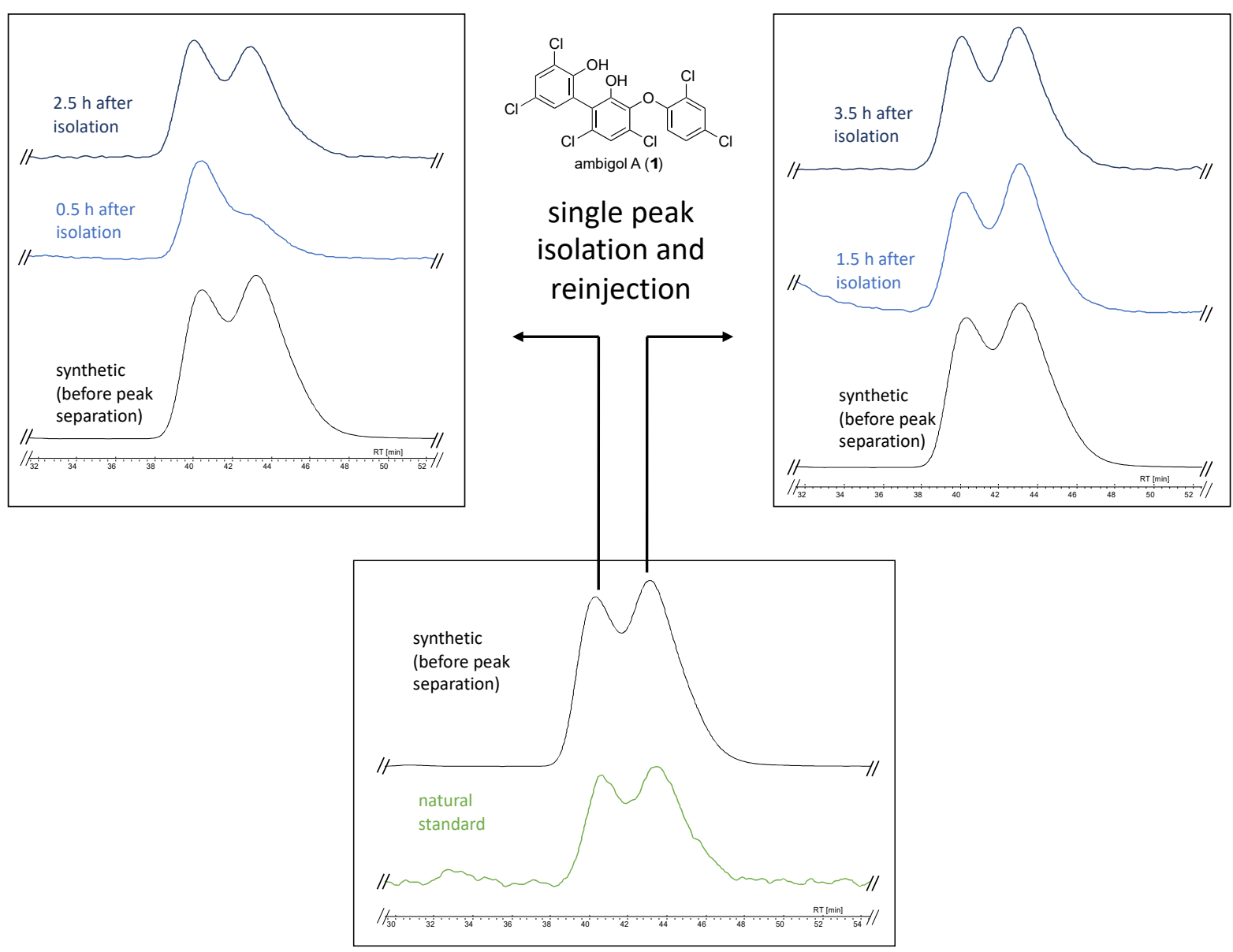

Fig. S96 Chiral HPLC analysis of ambigol A (1) including isolated standard from Fischerella ambigua. 


\section{References}

(1) Carreras, V.; Sandtorv, A. H.; Stuart, D. R. Synthesis of $\operatorname{Aryl}(2,4,6-$ trimethoxyphenyl)iodonium Trifluoroacetate Salts J. Org. Chem. 2017, 82, 1279-1284.

(2) Seidel, T. L.; Sundalam, S. K.; McCulloug, B.; Stuart, D. R. Unsymmetrical Aryl(2,4,6trimethoxyphenyl)iodonium Salts: One-Pot Synthesis, Scope, Stability, and Synthetic Studies J. Org. Chem. 2016, 81, 1998-2009.

(3) Jalalian, N.; Ishikawa, E. E.; Silva, L. F.; Olofsson, B. Room Temperature, Metal-Free Synthesis of Diaryl Ethers with Use of Diaryliodonium Salts Org. Lett. 2011, 13, 1552-1555.

(4) Falch, B. S.; Koenig, G. M.; Wright, A. D.; Sticher, O.; Ruegger, H.; Bernardinelli, G. Ambigol $A$ and B: New Biologically Active Polychlorinated Aromatic Compounds from the Terrestrial Blue-Green Alga Fischerella ambigua J. Org. Chem. 1993, 58, 6570-6575.

(5) Francke, R.; Schnakenburg, G.;Waldvogel, S. R. Efficient and Reliable lodination and OMethylation of Fluorinated Phenols Eur. J. Org. Chem. 2010, 12, 2357-2362.

(6) Duell, E. R.; Milzarek, T. M.; Omari, M. E.; Otoya, L. L.; Schäberle, T. F.; König, G. M.; Gulder, T. A. M. Identification, Cloning, Expression and Functional Interrogation of Biosynthetic Pathway of the Polychlorinated Triphenyls Ambigol A-C from Fischerella ambigua 108b Org. Chem. Front. 2020, 7, 3193-3201.

(7) Schmidt, B.; Riemer, M. Suzuki-Miyaura Coupling of Halophenols and Phenol Boronic Acids: Systematic Investigation of Positional Isomer Effects and Conclusions for the Synthesis of Phytoalexins from Pyrinae J. Org. Chem. 2014, 79, 4104-4118.

(8) Kitamura, Y.; Sako, S.; Udzu, T.; Tsutsui, A.; Maegawa, T.; Monguchi; Y.; Sajiki, H. Ligand-free $\mathrm{Pd} / \mathrm{C}$-catalyzed Suzuki-Miyaura coupling reaction for the synthesis of heterobiaryl derivatives Chem. Comm. 2007, 47, 5069-5071.

(9) Montgomery, T. P.; Grandner, J. M.; Houk, K. N.; Grubbs, R. H. Synthesis and Evaluation of Sterically Demanding Ruthenium Dithiolate Catalysts for Stereoretentive Olefin Metathesis Organometallics 2017, 36, 3940-3953.

(10)WO patent WO2014/202580 A1, 2014, Spiro-substituted Oxindole Derivatives having AMPK Activity.

(11)Baker, M. B.; Ferreira, R. B.; Tasseroul, J.; Lampkins, A. J.; Al Abbas, A.; Abboud, K. A.; Castellano, R. K. Selective and Seuqnetial Aminolysis of Bezotrifuranone: Synergism of Electronic Effects and Ring Strain Gradient J. Org. Chem. 2016, 81, 9279-9288.

(12)Tang, R.-J.; Milcent, T.; Crousse, B. Regioselective Halogenation of Arenes and Heterocycles in Hexyfluoroisopropanol J. Org. Chem. 2018, 83, 930-938.

(13)Chilczuk, T.; Monson, R.; Schmieder, P.; Christov, V.; Enke, H.; Salmond, G.; Niedermeyer, T. $\mathrm{H}$. J. Ambigols from the Cyanobacterium Fischerella ambigua increase Prodigiosin Production in Serratia spp ACS Chem. Biol. Article ASAP, DOI: 10.1021/acschembio.0c00554.

(14)Carson, M. W.; Giese, M. W.; Coghlan, M. J. An Intra/Intermolecular Suzuki Sequence to Benzopyridyloxepines Containing Geometrically Pure Exocyclic Tetrasubstituted Alkenes Org. Lett. 2008, 10, 2701-2704.

(15)Zhou, X.-Y.; Chen, X. $\mathrm{Na}_{2} \mathrm{CO}_{3}$-Catalyzed O-Acylation of Phenols for the Synthesis of Aryl Carboxylates with Use of Alkenyl Carboxylates Synlett 2018, 29, 2321-2325. 